\title{
Calculation of Free-Energy Barriers with TD-DFT: A Case Study on Excited-State Proton Transfer in Indigo
}

Changfeng Fang*,† and Bo Durbeej*,\$

'Center for Optics Research and Engineering (CORE), Shandong University, Qingdao 266237, China

Division of Theoretical Chemistry, IFM, Linköping University, SE-581 83 Linköping, Sweden

Supporting Information

Corresponding Authors

*E-mail: cfang@sdu.edu.cn (C.F.)

*E-mail: bodur@ifm.liu.se (B.D.) 


\section{CONTENTS}

$\begin{array}{ll}\text { Full citation for ref } 117 & \text { Page S3 }\end{array}$

$\begin{array}{ll}\text { Table S1 } & \text { Page S4 }\end{array}$

$\begin{array}{ll}\text { Table S2 Page S5 } & \text { P }\end{array}$

Table S3 Page S6

$\begin{array}{ll}\text { Table S4 } & \text { Page S7 }\end{array}$

$\begin{array}{ll}\text { Table S5 } & \text { Page S8 }\end{array}$

$\begin{array}{ll}\text { Table S6 } & \text { Page S9 }\end{array}$

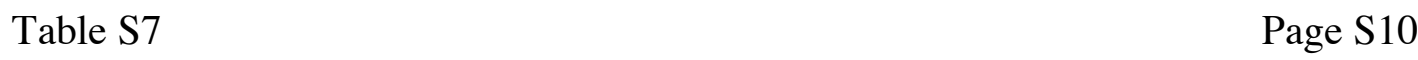

$\begin{array}{ll}\text { Table S8 } & \text { Page S12 }\end{array}$

$\begin{array}{ll}\text { Table S9 } & \text { Page S13 }\end{array}$

$\begin{array}{ll}\text { Table S10 Page S15 } & \text { Page S17 }\end{array}$

$\begin{array}{ll}\text { Table S11 } & \text { Page S17 }\end{array}$

$\begin{array}{ll}\text { Table S12 } & \text { Page S19 }\end{array}$

$\begin{array}{ll}\text { Table S13 } & \text { Page S20 }\end{array}$

$\begin{array}{ll}\text { Figure S1 Page S21 } & \text { P }\end{array}$

$\begin{array}{lr}\text { Figure S2 } & \text { Page S22 }\end{array}$

$\begin{array}{ll}\text { Figure S3 Page S23 } & \text { Pae S24 }\end{array}$

Cartesian coordinates for optimized $S_{0}$ and $S_{1}$ geometries $\quad$ Page $S 24$

B3LYP/6-311G(d,p) geometries Page S24

CC2/6-311G(d,p) geometries Page S54

Cartesian coordinates for $\mathrm{S}_{0}$ and $\mathrm{S}_{1}$ IRC geometries $\quad$ Page S66

B3LYP/6-311G(d,p) geometries Page S66

$\omega B 97 X-D / 6-311 G(d, p)$ geometries $\quad$ Page S116 


\section{FULL CITATION FOR REF 117}

(117) Frisch, M. J.; Trucks, G. W.; Schlegel, H. B.; Scuseria, G. E.; Robb, M. A.; Cheeseman, J. R.; Scalmani, G.; Barone, V.; Petersson, G. A.; Nakatsuji, H.; Li, X.; Caricato, M.; Marenich, A. V.; Bloino, J.; Janesko, B. G.; Gomperts, R.; Mennucci, B.; Hratchian, H. P.; Ortiz, J. V .; Izmaylov, A. F.; Sonnenberg, J. L.; Williams-Young, D.; Ding, F.; Lipparini, F.; Egidi, F.; Goings, J.; Peng, B.; Petrone, A.; Henderson, T.; Ranasinghe, D.; Zakrzewski, V. G.; Gao, J.; Rega, N.; Zheng, G.; Liang, W.; Hada, M.; Ehara, M.; Toyota, K.; Fukuda, R.; Hasegawa, J.; Ishida, M.; Nakajima, T.; Honda, Y.; Kitao, O.; Nakai, H.; Vreven, T.; Throssell, K.; Montgomery, J. A., Jr.; Peralta, J. E.; Ogliaro, F.; Bearpark, M. J.; Heyd, J. J.; Brothers, E. N.; Kudin, K. N.; Staroverov, V. N.; Keith, T. A.; Kobayashi, R.; Normand, J.; Raghavachari, K.; Rendell, A. P.; Burant, J. C.; Iyengar, S. S.; Tomasi, J.; Cossi, M.; Millam, J. M.; Klene, M.; Adamo, C.; Cammi, R.; Ochterski, J. W.; Martin, R. L.; Morokuma, K.; Farkas, O.; Foresman, J. B.; Fox, D. J. Gaussian 16, Revision B.01; Gaussian, Inc.: Wallingford, CT, 2016. 


\begin{tabular}{|c|c|c|c|c|c|c|c|c|c|c|c|}
\hline \multirow[b]{2}{*}{ molecule } & \multirow[b]{2}{*}{ reaction } & \multirow[b]{2}{*}{ energy $^{b}$} & \multicolumn{9}{|c|}{ method } \\
\hline & & & BP86 & B3LYP & PBE0 & M06-2X & MN15 & $\mathrm{CAM}^{c}$ & $\omega B 97 X-D$ & $\mathrm{HF} / \mathrm{CIS}$ & $\mathrm{CC} 2$ \\
\hline \multirow[t]{4}{*}{ Ind } & forward & $E$ & 7.0 & 7.5 & 4.9 & 6.7 & 6.2 & 6.8 & 7.8 & 12.6 & 2.5 \\
\hline & ESPT & $E_{0}$ & 4.4 & 4.8 & 3.8 & 4.0 & 3.9 & 4.2 & 5.0 & 9.3 & -0.1 \\
\hline & & $G$ & 4.8 & 5.3 & 4.0 & 4.4 & 4.5 & 4.7 & 5.5 & 9.7 & -1.9 \\
\hline & & $v^{d}$ & 1067 & 1304 & 1209 & 1180 & 1318 & 1303 & 1332 & 1806 & 754 \\
\hline \multirow[t]{4}{*}{ Ind } & reverse & $E$ & 2.5 & 5.8 & 5.3 & 8.4 & 8.5 & 7.4 & 7.9 & 19.6 & 4.4 \\
\hline & ESPT & $E_{0}$ & 0.3 & 3.0 & 2.5 & 5.2 & 5.4 & 4.2 & 4.6 & 15.8 & 1.9 \\
\hline & & $G$ & 0.5 & 3.3 & 2.8 & 5.3 & 5.6 & 4.4 & 4.8 & 16.0 & 1.4 \\
\hline & & $v^{d}$ & 1067 & 1304 & 1209 & 1180 & 1318 & 1303 & 1332 & 1806 & 754 \\
\hline \multirow[t]{4}{*}{ Ind } & forward & $E$ & 10.8 & 14.7 & 13.1 & 14.7 & 14.8 & 15.3 & 16.1 & 25.3 & 9.9 \\
\hline & GSPT & $E_{0}$ & 8.0 & 11.8 & 10.2 & 11.9 & 12.0 & 12.5 & 13.2 & 22.5 & 7.0 \\
\hline & & $G$ & 8.5 & 12.2 & 10.6 & 12.2 & 12.3 & 12.9 & 13.6 & 23.4 & 6.1 \\
\hline & & $v^{d}$ & 1011 & 1390 & 1298 & 1460 & 1545 & 1455 & 1478 & 1987 & 1211 \\
\hline \multirow[t]{4}{*}{ Ind } & reverse & $E$ & 1.6 & 4.6 & 3.7 & 6.8 & 6.4 & 5.4 & 5.8 & 15.1 & 3.5 \\
\hline & GSPT & $E_{0}$ & -1.0 & 1.8 & 0.9 & 3.7 & 3.4 & 2.4 & 2.7 & 11.6 & 0.8 \\
\hline & & $G$ & -0.6 & 2.0 & 1.1 & 3.8 & 3.7 & 2.7 & 2.9 & 11.8 & -0.5 \\
\hline & & $v^{d}$ & 1011 & 1390 & 1298 & 1460 & 1545 & 1455 & 1478 & 1987 & 1211 \\
\hline \multirow[t]{4}{*}{ NHxInd } & forward & $E$ & 5.4 & 5.6 & 4.0 & 5.0 & 4.8 & 5.2 & 5.9 & 9.1 & 1.9 \\
\hline & ESPT & $E_{0}$ & 2.9 & 3.0 & 1.6 & 1.9 & 2.2 & 2.4 & 2.9 & 5.8 & -0.8 \\
\hline & & $G$ & 3.2 & 3.4 & 2.0 & 1.9 & 2.5 & 2.5 & 3.0 & 5.8 & -1.1 \\
\hline & & $v^{d}$ & 739 & 1180 & 1101 & 1204 & 1290 & 1267 & 1310 & 1786 & 984 \\
\hline \multirow[t]{4}{*}{ NHxInd } & reverse & $E$ & 4.8 & 3.1 & 2.5 & 4.2 & 4.6 & 3.6 & 3.9 & 13.5 & 3.1 \\
\hline & ESPT & $E_{0}$ & 2.1 & 0.6 & 0.0 & 1.3 & 1.8 & 0.7 & 0.8 & 9.9 & -0.8 \\
\hline & & $G$ & 2.1 & 0.7 & 0.1 & 1.5 & 2.0 & 0.9 & 1.2 & 10.0 & 0.7 \\
\hline & & $v^{d}$ & 739 & 1180 & 1101 & 1204 & 1290 & 1267 & 1310 & 1786 & 984 \\
\hline \multirow[t]{4}{*}{ NHxInd } & forward & $E$ & 7.9 & 10.7 & 9.3 & 11.0 & 11.2 & 11.4 & 12.1 & 20.4 & 6.3 \\
\hline & GSPT & $E_{0}$ & 5.6 & 7.9 & 6.5 & 8.1 & 8.3 & 8.6 & 9.2 & 17.3 & 3.6 \\
\hline & & $G$ & 5.9 & 8.2 & 6.5 & 8.0 & 8.1 & 8.3 & 9.1 & 17.1 & 3.3 \\
\hline & & $v^{d}$ & 698 & 1187 & 1049 & 1358 & 1404 & 1288 & 1315 & 1894 & 1070 \\
\hline \multirow[t]{4}{*}{ NHxInd } & reverse & $E$ & 0.0 & 1.8 & 1.1 & 3.5 & 3.4 & 2.4 & 2.5 & 10.3 & 1.6 \\
\hline & GSPT & $E_{0}$ & -1.2 & -0.9 & -1.4 & 0.6 & 0.5 & -0.4 & -0.3 & 6.8 & -1.2 \\
\hline & & $G$ & -0.8 & -0.8 & -1.4 & 0.6 & 0.3 & -0.6 & -0.3 & 6.5 & -1.0 \\
\hline & & $v^{d}$ & 698 & 1187 & 1049 & 1358 & 1404 & 1288 & 1315 & 1894 & 1070 \\
\hline \multicolumn{12}{|c|}{$\begin{array}{l}{ }^{a} \text { All CC2 calculations carried out with the } 6-311++\mathrm{G}(\mathrm{d}, \mathrm{p}) \text { basis set, all other calculations carried out with the } 6-311+\mathrm{G}(\mathrm{d}, \mathrm{p}) \text { basis set. } \\
{ }^{b} \text { Barriers given in terms of electronic energies }(E) \text {, electronic energies plus ZPVE corrections }\left(E_{0}\right) \text {, and free energies }(G){ }^{c}{ }^{C} \mathrm{CAM}-\mathrm{B} 3 \mathrm{LYP} \text {. }\end{array}$} \\
\hline
\end{tabular}




\begin{tabular}{|c|c|c|c|c|c|c|c|c|c|c|c|}
\hline \multicolumn{12}{|c|}{$\begin{array}{l}\text { Table S2. Changes in Calculated Energy Barriers for Forward and Reverse ESPT and GSPT in Indigo (Ind) and } N \text {-Hexylindigo } \\
\left.\text { (NHxInd) in the Gas Phase Upon Inclusion of ZPVE and Thermal Corrections (in kcal } \mathrm{mol}^{-1}\right)^{a}\end{array}$} \\
\hline \multirow{2}{*}{$\begin{array}{l}\text { molecule / } \\
\text { reaction }\end{array}$} & \multirow[b]{2}{*}{ change $^{b}$} & \multicolumn{9}{|c|}{ method } & \multirow[b]{2}{*}{$\mathbf{S D}^{d}$} \\
\hline & & BP86 & B3LYP & PBE0 & M06-2X & MN15 & $\mathrm{CAM}^{c}$ & $\omega \mathrm{B} 97 \mathrm{X}-\mathrm{D}$ & HF/CIS & $\overline{\mathrm{C}} \mathrm{C} 2$ & \\
\hline Ind & $E \rightarrow E_{0}$ & -2.6 & -2.7 & -1.1 & -2.7 & -2.3 & -2.6 & -2.8 & -3.3 & -2.6 & 0.60 \\
\hline forward ESPT & $E_{0} \rightarrow G$ & 0.4 & 0.5 & 0.2 & 0.4 & 0.6 & 0.5 & 0.5 & 0.4 & -1.8 & 0.75 \\
\hline Ind & $E \rightarrow E_{0}$ & -2.2 & -2.8 & -2.8 & -3.2 & -3.1 & -3.2 & -3.3 & -3.8 & -2.5 & 0.47 \\
\hline reverse ESPT & $E_{0} \rightarrow G$ & 0.2 & 0.3 & 0.3 & 0.1 & 0.2 & 0.2 & 0.2 & 0.2 & -0.5 & 0.24 \\
\hline Ind & $E \rightarrow E_{0}$ & -2.8 & -2.9 & -2.9 & -2.8 & -2.8 & -2.8 & -2.9 & -2.8 & -2.9 & 0.05 \\
\hline forward GSPT & $E_{0} \rightarrow G$ & 0.5 & 0.4 & 0.4 & 0.3 & 0.3 & 0.4 & 0.4 & 0.9 & -0.9 & 0.48 \\
\hline Ind & $E \rightarrow E_{0}$ & -2.6 & -2.8 & -2.8 & -3.1 & -3 & -3 & -3.1 & -3.5 & -2.7 & 0.27 \\
\hline reverse GSPT & $E_{0} \rightarrow G$ & 0.4 & 0.2 & 0.2 & 0.1 & 0.3 & 0.3 & 0.2 & 0.2 & -1.3 & 0.52 \\
\hline NHxInd & $E \rightarrow E_{0}$ & -2.5 & -2.6 & -2.4 & -3.1 & -2.6 & -2.8 & -3 & -3.3 & -2.7 & 0.30 \\
\hline forward ESPT & $E_{0} \rightarrow G$ & 0.3 & 0.4 & 0.4 & 0 & 0.3 & 0.1 & 0.1 & 0 & -0.3 & 0.23 \\
\hline NHxInd & $E \rightarrow E_{0}$ & -2.7 & -2.5 & -2.5 & -2.9 & -2.8 & -2.9 & -3.1 & -3.6 & -3.9 & 0.48 \\
\hline reverse ESPT & $E_{0} \rightarrow G$ & 0 & 0.1 & 0.1 & 0.2 & 0.2 & 0.2 & 0.4 & 0.1 & 1.5 & 0.46 \\
\hline NHxInd & $E \rightarrow E_{0}$ & -2.3 & -2.8 & -2.8 & -2.9 & -2.9 & -2.8 & -2.9 & -3.1 & -2.7 & 0.22 \\
\hline forward GSPT & $E_{0} \rightarrow G$ & 0.3 & 0.3 & 0 & -0.1 & -0.2 & -0.3 & -0.1 & -0.2 & -0.3 & 0.23 \\
\hline NHxInd & $E \rightarrow E_{0}$ & -1.2 & -2.7 & -2.5 & -2.9 & -2.9 & -2.8 & -2.8 & -3.5 & -2.8 & 0.62 \\
\hline reverse GSPT & $E_{0} \rightarrow G$ & 0.4 & 0.1 & 0 & 0 & -0.2 & -0.2 & 0 & -0.3 & 0.2 & 0.22 \\
\hline
\end{tabular}




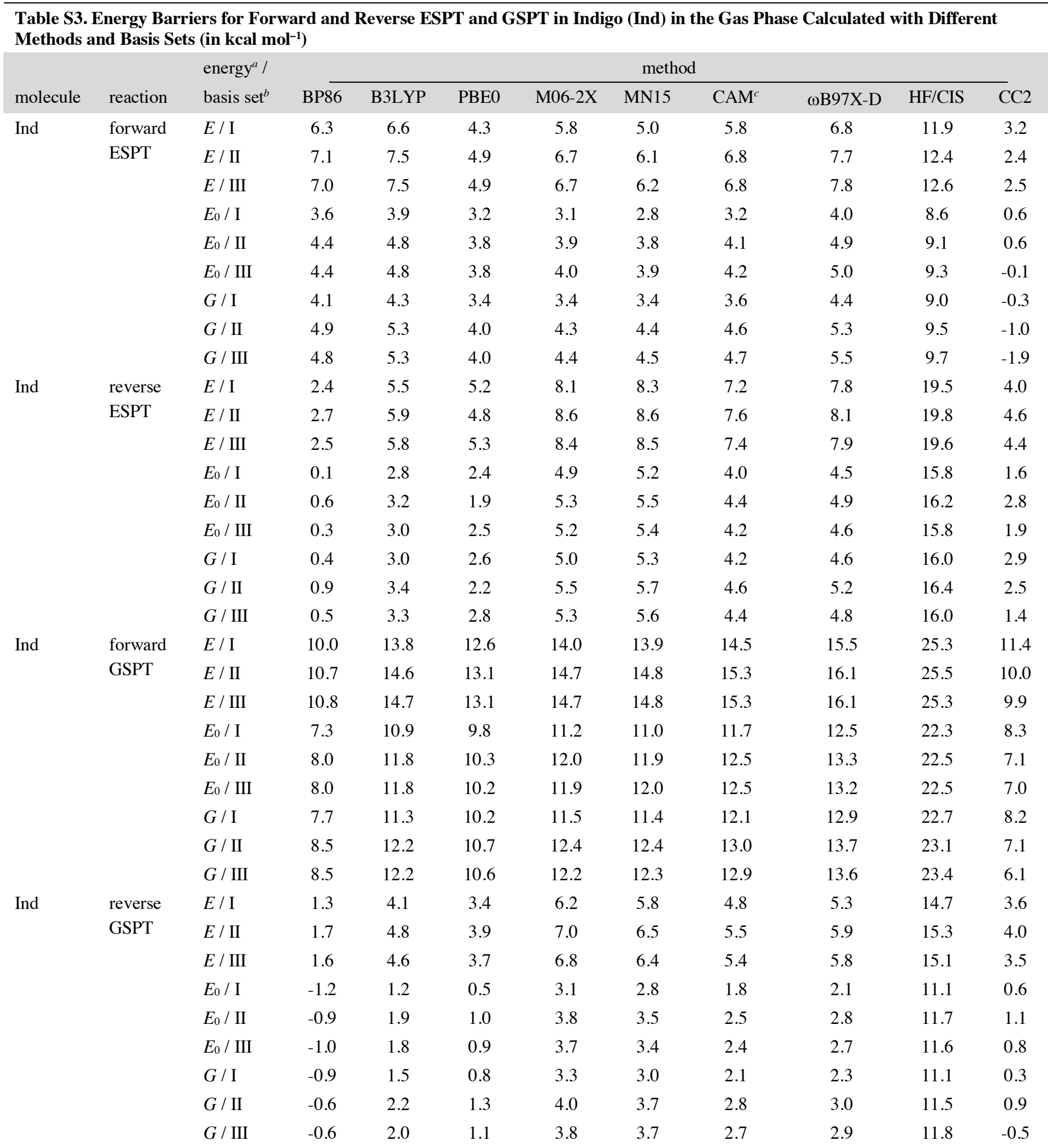

${ }^{a}$ Barriers given in terms of electronic energies $(E)$, electronic energies plus ZPVE corrections $\left(E_{0}\right)$, and free energies $(G)$. ${ }^{b} \mathrm{I}$, II and III denote the 6-31G(d,p), 6-311G(d,p) and 6-311+G(d,p) (6-311++G(d,p) for CC2) basis sets, respectively. ${ }^{c}$ CAM-B3LYP. 


\begin{tabular}{|c|c|c|c|c|c|c|c|c|c|c|c|}
\hline \multicolumn{12}{|c|}{$\begin{array}{l}\text { Table S4. Energy Barriers for Forward and Reverse ESPT and GSPT in } N \text {-Hexylindigo (NHxInd) in the Gas Phase Calculated with } \\
\text { Different Methods and Basis Sets (in kcal mol-1) }\end{array}$} \\
\hline \multirow[b]{2}{*}{ molecule } & \multirow[b]{2}{*}{ reaction } & \multirow{2}{*}{$\begin{array}{l}\text { energy }^{a} / \\
\text { basis set }^{b}\end{array}$} & \multirow[b]{2}{*}{ BP86 } & \multirow[b]{2}{*}{ B3LYP } & \multirow[b]{2}{*}{ PBE0 } & \multicolumn{3}{|c|}{ method } & \multirow[b]{2}{*}{$\omega B 97 X-D$} & \multirow[b]{2}{*}{ HF/CIS } & \multirow[b]{2}{*}{$\mathrm{CC} 2$} \\
\hline & & & & & & M06-2X & MN15 & $\mathrm{CAM}^{c}$ & & & \\
\hline \multirow[t]{9}{*}{ NHxInd } & forward & $E / \mathrm{I}$ & 4.9 & 4.9 & 3.7 & 3.9 & 3.6 & 4.1 & 5.0 & 8.4 & 2.5 \\
\hline & ESPT & $E / \mathrm{II}$ & 5.6 & 5.7 & 4.1 & 4.8 & 4.6 & 5.0 & 5.8 & 8.8 & 1.7 \\
\hline & & $E$ / III & 5.4 & 5.6 & 4.0 & 5.0 & 4.8 & 5.2 & 5.9 & 9.1 & 1.6 \\
\hline & & $E_{0} / \mathrm{I}$ & 2.4 & 2.3 & 1.2 & 1.1 & 1.2 & 1.4 & 2.2 & 5.1 & -0.3 \\
\hline & & $E_{0} / \mathrm{II}$ & 3.0 & 3.0 & 1.6 & 1.9 & 2.1 & 2.2 & 2.9 & 5.6 & -0.9 \\
\hline & & $E_{0} /$ III & 2.9 & 3.0 & 1.6 & 1.9 & 2.2 & 2.4 & 2.9 & 5.8 & -1.2 \\
\hline & & $G / \mathrm{I}$ & 2.7 & 2.5 & 1.4 & 1.3 & 1.2 & 1.4 & 2.5 & 5.2 & -1.3 \\
\hline & & $G$ / II & 3.3 & 3.3 & 1.9 & 2.0 & 2.3 & 2.3 & 3.1 & 5.7 & -1.4 \\
\hline & & $G / \mathrm{III}$ & 3.2 & 3.4 & 2.0 & 1.9 & 2.5 & 2.5 & 3.0 & 5.8 & -1.0 \\
\hline \multirow[t]{9}{*}{ NHxInd } & reverse & $E / \mathrm{I}$ & 4.2 & 2.4 & 2.0 & 3.8 & 4.1 & 3.2 & 3.6 & 13.3 & 2.4 \\
\hline & ESPT & $E / \mathrm{II}$ & 4.8 & 3.1 & 2.5 & 4.2 & 4.6 & 3.6 & 3.9 & 13.7 & 3.1 \\
\hline & & $E$ / III & 4.8 & 3.1 & 2.5 & 4.2 & 4.6 & 3.6 & 3.9 & 13.5 & 3.1 \\
\hline & & $E_{0} / \mathrm{I}$ & 1.7 & 0.0 & -0.2 & 0.9 & 1.3 & 0.4 & 0.5 & 9.7 & -1.1 \\
\hline & & $E_{0} / \mathrm{II}$ & 2.1 & 0.5 & 0.0 & 1.3 & 1.7 & 0.7 & 0.8 & 10.2 & -0.8 \\
\hline & & $E_{0} /$ III & 2.1 & 0.6 & 0.0 & 1.3 & 1.8 & 0.7 & 0.8 & 9.9 & -0.8 \\
\hline & & $G / \mathrm{I}$ & 1.6 & -0.1 & -0.3 & 1.0 & 1.3 & 0.4 & 0.6 & 10.0 & -0.4 \\
\hline & & $G / \mathrm{II}$ & 2.1 & 0.5 & 0.0 & 1.3 & 1.8 & 0.8 & 1.1 & 10.4 & 0.7 \\
\hline & & $G / \mathrm{III}$ & 2.1 & 0.7 & 0.1 & 1.5 & 2.0 & 0.9 & 1.2 & 10.0 & 0.7 \\
\hline \multirow[t]{9}{*}{ NHxInd } & forward & $E / \mathrm{I}$ & $-^{d}$ & 10.0 & 9.0 & 10.1 & 10.2 & 10.7 & 11.5 & 20.2 & 8.0 \\
\hline & GSPT & $E / \mathrm{II}$ & 7.8 & 10.8 & 9.4 & 11.0 & 11.2 & 11.5 & 12.2 & 20.5 & 6.7 \\
\hline & & $E$ / III & 7.9 & 10.7 & 9.3 & 11.0 & 11.2 & 11.4 & 12.1 & 20.4 & 6.3 \\
\hline & & $E_{0} / \mathrm{I}$ & $-^{d}$ & 7.1 & 6.2 & 7.3 & 7.3 & 7.8 & 8.8 & 17.1 & 5.1 \\
\hline & & $E_{0} / \mathrm{II}$ & 5.5 & 8.0 & 6.6 & 8.2 & 8.3 & 8.7 & 9.4 & 17.4 & 3.9 \\
\hline & & $E_{0} /$ III & 5.6 & 7.9 & 6.5 & 8.1 & 8.3 & 8.6 & 9.2 & 17.3 & 3.6 \\
\hline & & $G / \mathrm{I}$ & $-^{d}$ & 7.3 & 6.3 & 7.3 & 7.1 & 8.0 & 9.2 & 16.9 & 5.0 \\
\hline & & $G / \mathrm{II}$ & 5.9 & 8.2 & 6.7 & 8.2 & 8.2 & 8.8 & 9.7 & 17.2 & 3.9 \\
\hline & & $G / \mathrm{III}$ & 5.9 & 8.2 & 6.5 & 8.0 & 8.1 & 8.3 & 9.1 & 17.1 & 3.3 \\
\hline \multirow[t]{9}{*}{ NHxInd } & reverse & $E / \mathrm{I}$ & $-^{d}$ & 1.2 & 0.8 & 2.7 & 2.7 & 1.8 & 2.1 & 9.6 & 0.8 \\
\hline & GSPT & $E / \mathrm{II}$ & 0.0 & 1.7 & 1.1 & 3.4 & 3.3 & 2.4 & 2.6 & 10.3 & 1.4 \\
\hline & & $E /$ III & 0.0 & 1.8 & 1.1 & 3.5 & 3.4 & 2.4 & 2.5 & 10.3 & 1.6 \\
\hline & & $E_{0} / \mathrm{I}$ & $-^{d}$ & -1.3 & -1.6 & 0.0 & -0.2 & -0.8 & -0.7 & 6.2 & -1.8 \\
\hline & & $E_{0} / \mathrm{II}$ & -1.0 & -0.9 & -1.3 & 0.6 & 0.4 & -0.4 & -0.2 & 6.8 & -1.7 \\
\hline & & $E_{0} /$ III & -1.2 & -0.9 & -1.4 & 0.6 & 0.5 & -0.4 & -0.3 & 6.8 & -1.2 \\
\hline & & $G / \mathrm{I}$ & $-^{d}$ & -1.1 & -1.4 & 0.0 & -0.4 & -0.7 & -0.6 & 5.9 & -1.3 \\
\hline & & $G$ / II & -0.6 & -0.7 & -1.2 & 0.5 & 0.4 & -0.2 & 0.0 & 6.5 & -0.9 \\
\hline & & $G / \mathrm{III}$ & -0.8 & -0.8 & -1.4 & 0.6 & 0.3 & -0.6 & -0.3 & 6.5 & -1.0 \\
\hline
\end{tabular}


Table S5. Energy Barriers for Forward and Reverse ESPT and GSPT in Indigo (Ind) Calculated with Different Methods in Different Media (in kcal mol $\left.{ }^{-1}\right)^{a}$

\begin{tabular}{|c|c|c|c|c|c|c|c|c|c|c|}
\hline \multirow[b]{2}{*}{ molecule } & \multirow[b]{2}{*}{ reaction } & \multirow{2}{*}{$\begin{array}{l}\text { energy }^{b} / \\
\text { medium }^{c}\end{array}$} & \multirow[b]{2}{*}{ BP86 } & \multicolumn{6}{|c|}{ method } & \multirow[b]{2}{*}{$\mathrm{HF} / \mathrm{CIS}$} \\
\hline & & & & B3LYP & PBE0 & M06-2X & MN15 & $\mathrm{CAM}^{d}$ & $\omega B 97 X-D$ & \\
\hline \multirow[t]{6}{*}{ Ind } & forward & $E / \mathrm{GAS}$ & 7.1 & 7.5 & 4.9 & 6.7 & 6.1 & 6.8 & 7.7 & 12.4 \\
\hline & ESPT & $E / \mathrm{THF}$ & 6.8 & 9.3 & 7.9 & 8.9 & 8.8 & 9.3 & 10.1 & 15.2 \\
\hline & & $E_{0} / \mathrm{GAS}$ & 4.4 & 4.8 & 3.8 & 3.9 & 3.8 & 4.1 & 4.9 & 9.1 \\
\hline & & $E_{0} / \mathrm{THF}$ & 4.4 & 6.6 & 5.1 & 6.1 & 5.9 & 6.4 & 7.2 & 12.3 \\
\hline & & $G / \mathrm{GAS}$ & 4.9 & 5.3 & 4.0 & 4.3 & 4.4 & 4.6 & 5.3 & 9.5 \\
\hline & & $G / \mathrm{THF}$ & 5.0 & 7.4 & 5.8 & 6.9 & 6.7 & 7.1 & 8.1 & 13.3 \\
\hline \multirow[t]{6}{*}{ Ind } & reverse & $E$ / GAS & 2.7 & 5.9 & 4.8 & 8.6 & 8.6 & 7.6 & 8.1 & 19.8 \\
\hline & ESPT & $E / \mathrm{THF}$ & 3.3 & 5.7 & 4.9 & 6.5 & 7.2 & 5.8 & 6.0 & 16.0 \\
\hline & & $E_{0} / \mathrm{GAS}$ & 0.6 & 3.2 & 1.9 & 5.3 & 5.5 & 4.4 & 4.9 & 16.2 \\
\hline & & $E_{0} / \mathrm{THF}$ & 0.5 & 2.7 & 1.8 & 3.5 & 4.1 & 2.8 & 3.0 & 12.8 \\
\hline & & $G$ / GAS & 0.9 & 3.4 & 2.2 & 5.5 & 5.7 & 4.6 & 5.2 & 16.4 \\
\hline & & $G / \mathrm{THF}$ & 0.7 & 2.9 & 2.0 & 3.7 & 4.3 & 3.0 & 3.2 & 13.1 \\
\hline \multirow[t]{6}{*}{ Ind } & forward & $E / \mathrm{GAS}$ & 10.7 & 14.6 & 13.1 & 14.7 & 14.8 & 15.3 & 16.1 & 25.5 \\
\hline & GSPT & $E / \mathrm{THF}$ & 11.4 & 15.3 & 13.7 & 15.2 & 15.3 & 15.8 & 16.7 & 26.0 \\
\hline & & $E_{0} / \mathrm{GAS}$ & 8.0 & 11.8 & 10.3 & 12.0 & 11.9 & 12.5 & 13.3 & 22.5 \\
\hline & & $E_{0} / \mathrm{THF}$ & 8.9 & 12.6 & 11.1 & 12.6 & 12.7 & 13.3 & 14.1 & 23.4 \\
\hline & & $G / \mathrm{GAS}$ & 8.5 & 12.2 & 10.7 & 12.4 & 12.4 & 13.0 & 13.7 & 23.1 \\
\hline & & $G / \mathrm{THF}$ & 9.7 & 13.3 & 11.7 & 13.2 & 13.3 & 13.9 & 14.8 & 23.6 \\
\hline \multirow[t]{6}{*}{ Ind } & reverse & $E$ / GAS & 1.7 & 4.8 & 3.9 & 7.0 & 6.5 & 5.5 & 5.9 & 15.3 \\
\hline & GSPT & $E / \mathrm{THF}$ & 1.0 & 4.0 & 3.1 & 6.2 & 5.7 & 4.8 & 5.2 & 14.4 \\
\hline & & $E_{0} / \mathrm{GAS}$ & -0.9 & 1.9 & 1.0 & 3.8 & 3.5 & 2.5 & 2.8 & 11.7 \\
\hline & & $E_{0} / \mathrm{THF}$ & -1.4 & 1.1 & 0.2 & 3.0 & 2.7 & 1.8 & 2.1 & 10.8 \\
\hline & & $G / \mathrm{GAS}$ & -0.6 & 2.2 & 1.3 & 4.0 & 3.7 & 2.8 & 3.0 & 11.5 \\
\hline & & $G / \mathrm{THF}$ & -1.1 & 1.4 & 0.6 & 3.2 & 2.9 & 2.1 & 2.3 & 11.0 \\
\hline
\end{tabular}

${ }^{a}$ All calculations carried out with the $6-311 \mathrm{G}(\mathrm{d}, \mathrm{p})$ basis set. ${ }^{b}$ Barriers given in terms of electronic energies $(E)$, electronic energies plus ZPVE corrections $\left(E_{0}\right)$, and free energies $(G) .{ }^{c} \mathrm{GAS}$ and THF denote gas phase and tetrahydrofuran, respectively. ${ }^{d} \mathrm{CAM}-\mathrm{B} 3 \mathrm{LYP}$. 
Table S6. Energy Barriers for Forward and Reverse ESPT and GSPT in $N$-Hexylindigo (NHxInd) Calculated with Different Methods in Different Media (in kcal mol-1) ${ }^{a}$

\begin{tabular}{|c|c|c|c|c|c|c|c|c|c|c|}
\hline \multirow[b]{2}{*}{ molecule } & \multirow[b]{2}{*}{ reaction } & \multirow{2}{*}{$\begin{array}{l}\text { energy }^{b} / \\
\text { medium }^{c}\end{array}$} & \multirow[b]{2}{*}{ BP86 } & \multicolumn{6}{|c|}{ method } & \multirow[b]{2}{*}{$\mathrm{HF} / \mathrm{CIS}$} \\
\hline & & & & B3LYP & PBE0 & M06-2X & MN15 & $\mathrm{CAM}^{d}$ & $\omega B 97 X-D$ & \\
\hline \multirow[t]{9}{*}{ NHxInd } & forward & $E / \mathrm{GAS}$ & 5.6 & 5.7 & 4.1 & 4.8 & 4.6 & 5.0 & 5.8 & 8.8 \\
\hline & ESPT & $E / \mathrm{MCH}$ & 5.3 & 5.8 & 4.7 & 5.5 & 5.5 & 5.9 & 6.6 & 9.9 \\
\hline & & $E / \mathrm{THF}$ & 4.2 & 6.7 & 5.4 & 6.3 & 6.3 & 6.7 & 7.5 & 11.2 \\
\hline & & $E_{0} / \mathrm{GAS}$ & 3.0 & 3.0 & 1.6 & 1.9 & 2.1 & 2.2 & 2.9 & 5.6 \\
\hline & & $E_{0} / \mathrm{MCH}$ & 2.5 & 3.5 & 2.1 & 2.5 & 2.6 & 2.9 & 3.6 & 6.7 \\
\hline & & $E_{0} / \mathrm{THF}$ & 1.8 & 3.9 & 2.7 & 3.3 & 3.4 & 3.8 & 4.4 & 8.3 \\
\hline & & $G$ / GAS & 3.3 & 3.3 & 1.9 & 2.0 & 2.3 & 2.3 & 3.1 & 5.7 \\
\hline & & $G / \mathrm{MCH}$ & 2.7 & 4.1 & 2.3 & 2.7 & 2.7 & 3.0 & 3.7 & 7.0 \\
\hline & & $G / \mathrm{THF}$ & 2.3 & 4.3 & 3.1 & 3.5 & 3.7 & 4.1 & 4.6 & 8.8 \\
\hline \multirow[t]{9}{*}{ NHxInd } & reverse & $E$ / GAS & 4.8 & 3.1 & 2.5 & 4.2 & 4.6 & 3.6 & 3.9 & 13.7 \\
\hline & ESPT & $E / \mathrm{MCH}$ & 3.8 & 3.2 & 2.6 & 3.6 & 4.3 & 3.1 & 3.3 & 12.2 \\
\hline & & $E / \mathrm{THF}$ & 2.0 & 3.0 & 2.2 & 2.8 & 3.7 & 2.4 & 2.6 & 10.6 \\
\hline & & $E_{0} / \mathrm{GAS}$ & 2.1 & 0.5 & 0.0 & 1.3 & 1.7 & 0.7 & 0.8 & 10.2 \\
\hline & & $E_{0} / \mathrm{MCH}$ & 1.1 & 0.4 & -0.3 & 0.8 & 1.3 & 0.3 & 0.3 & 8.8 \\
\hline & & $E_{0} / \mathrm{THF}$ & -0.7 & 0.0 & -0.5 & 0.3 & 0.9 & -0.2 & -0.1 & 7.4 \\
\hline & & $G / \mathrm{GAS}$ & 2.1 & 0.5 & 0.0 & 1.3 & 1.8 & 0.8 & 1.1 & 10.4 \\
\hline & & $G / \mathrm{MCH}$ & 1.1 & 0.5 & -0.4 & 0.8 & 1.2 & 0.4 & 0.4 & 9.0 \\
\hline & & $G / \mathrm{THF}$ & -0.6 & 0.3 & -0.2 & 0.3 & 0.9 & 0.1 & 0.1 & 7.8 \\
\hline \multirow[t]{9}{*}{ NHxInd } & forward & $E$ / GAS & 7.8 & 10.8 & 9.4 & 11.0 & 11.2 & 11.5 & 12.2 & 20.5 \\
\hline & GSPT & $E / \mathrm{MCH}$ & $-^{e}$ & 11.0 & 9.6 & 11.1 & 11.3 & 11.7 & 12.4 & 20.6 \\
\hline & & $E / \mathrm{THF}$ & $-^{e}$ & 11.4 & 9.9 & 11.5 & 11.7 & 11.9 & 12.6 & 20.9 \\
\hline & & $E_{0} / \mathrm{GAS}$ & 5.5 & 8.0 & 6.6 & 8.2 & 8.3 & 8.7 & 9.4 & 17.4 \\
\hline & & $E_{0} / \mathrm{MCH}$ & $-^{e}$ & 8.2 & 6.9 & 8.4 & 8.5 & 8.9 & 9.6 & 17.6 \\
\hline & & $E_{0} / \mathrm{THF}$ & $--^{e}$ & 8.7 & 7.3 & 8.7 & 8.9 & 9.3 & 9.9 & 18.0 \\
\hline & & $G / \mathrm{GAS}$ & 5.9 & 8.2 & 6.7 & 8.2 & 8.2 & 8.8 & 9.7 & 17.2 \\
\hline & & $G / \mathrm{MCH}$ & $-^{e}$ & 8.2 & 7.2 & 8.6 & 8.4 & 9.1 & 10.0 & 17.5 \\
\hline & & $G / \mathrm{THF}$ & $-^{e}$ & 9.2 & 6.3 & 8.8 & 8.9 & 9.5 & 10.0 & 18.2 \\
\hline \multirow[t]{9}{*}{ NHxInd } & reverse & $E /$ GAS & 0.0 & 1.7 & 1.1 & 3.4 & 3.3 & 2.4 & 2.6 & 10.3 \\
\hline & GSPT & $E / \mathrm{MCH}$ & $--^{e}$ & 1.5 & 0.9 & 3.2 & 3.1 & 2.2 & 2.4 & 10.0 \\
\hline & & $E / \mathrm{THF}$ & $-^{e}$ & 1.4 & 0.9 & 3.3 & 3.1 & 2.2 & 2.4 & 10.3 \\
\hline & & $E_{0} / \mathrm{GAS}$ & -1.0 & -0.9 & -1.3 & 0.6 & 0.4 & -0.4 & -0.2 & 6.8 \\
\hline & & $E_{0} / \mathrm{MCH}$ & $-^{e}$ & -1.1 & -1.5 & 0.5 & 0.3 & -0.5 & -0.3 & 6.5 \\
\hline & & $E_{0} / \mathrm{THF}$ & $-^{e}$ & -1.1 & -1.5 & 0.5 & 0.3 & -0.5 & -0.3 & 6.7 \\
\hline & & $G / \mathrm{GAS}$ & -0.6 & -0.7 & -1.2 & 0.5 & 0.4 & -0.2 & 0.0 & 6.5 \\
\hline & & $G / \mathrm{MCH}$ & $--^{e}$ & -1.0 & -1.1 & 0.6 & 0.1 & -0.5 & -0.3 & 6.2 \\
\hline & & $G / \mathrm{THF}$ & $-^{e}$ & -0.6 & -0.9 & 0.6 & 0.5 & -0.2 & -0.1 & 6.6 \\
\hline
\end{tabular}

${ }^{a}$ All calculations carried out with the $6-311 \mathrm{G}(\mathrm{d}, \mathrm{p})$ basis set. ${ }^{b}$ Barriers given in terms of electronic energies $(E)$, electronic energies plus ZPVE corrections $\left(E_{0}\right)$, and free energies $(G) .{ }^{c} \mathrm{GAS}, \mathrm{MCH}$ and THF denote gas phase, methylcyclohexane and tetrahydrofuran, respectively. ${ }^{d} \mathrm{CAM}-\mathrm{B} 3 \mathrm{LYP} .{ }^{e}$ Geometry optimization of $\mathrm{TS}_{12}$ failed to converge. 
Table S7. Energy Barriers for Forward and Reverse ESPT and GSPT in $N$-Hexylindigo (NHxInd) Calculated with Different Methods and with Full $\left(\mathrm{C}_{6} \mathrm{H}_{13}\right)$ and Reduced $\left(\mathrm{C}_{2} \mathrm{H}_{5}\right)$ Models of the $N$-Hexyl Substituent (in kcal mol$\left.{ }^{-1}\right)^{a}$

\begin{tabular}{|c|c|c|c|c|c|c|c|c|c|c|}
\hline \multirow[b]{2}{*}{ molecule } & \multirow[b]{2}{*}{ reaction } & \multirow{2}{*}{$\begin{array}{l}\text { energy }^{b} / \\
\text { medium }^{c}\end{array}$} & \multirow{2}{*}{$\begin{array}{l}N \text {-hexyl } \\
\text { model }\end{array}$} & \multicolumn{7}{|c|}{ method } \\
\hline & & & & B3LYP & PBE0 & M06-2X & MN15 & $\mathrm{CAM}^{d}$ & $\omega B 97 X-D$ & $\mathrm{HF} / \mathrm{CIS}$ \\
\hline \multirow[t]{12}{*}{ NHxInd } & \multirow{12}{*}{$\begin{array}{l}\text { forward } \\
\text { ESPT }\end{array}$} & \multirow[t]{2}{*}{$E / \mathrm{MCH}$} & $\mathrm{C}_{6} \mathrm{H}_{13}$ & 5.8 & 4.8 & 5.7 & 5.7 & 6.0 & 6.8 & 9.9 \\
\hline & & & $\mathrm{C}_{2} \mathrm{H}_{5}$ & 5.8 & 4.7 & 5.5 & 5.5 & 5.9 & 6.6 & 9.9 \\
\hline & & \multirow[t]{2}{*}{$E / \mathrm{THF}$} & $\mathrm{C}_{6} \mathrm{H}_{13}$ & 6.7 & 5.5 & 6.4 & 6.4 & 6.7 & 7.6 & 11.3 \\
\hline & & & $\mathrm{C}_{2} \mathrm{H}_{5}$ & 6.7 & 5.4 & 6.3 & 6.3 & 6.7 & 7.5 & 11.2 \\
\hline & & \multirow[t]{2}{*}{$E_{0} / \mathrm{MCH}$} & $\mathrm{C}_{6} \mathrm{H}_{13}$ & 3.7 & 2.2 & 2.7 & 2.7 & 3.0 & 4.1 & 6.8 \\
\hline & & & $\mathrm{C}_{2} \mathrm{H}_{5}$ & 3.5 & 2.1 & 2.5 & 2.6 & 2.9 & 3.6 & 6.7 \\
\hline & & \multirow[t]{2}{*}{$E_{0} / \mathrm{THF}$} & $\mathrm{C}_{6} \mathrm{H}_{13}$ & 3.9 & 2.8 & 3.5 & 3.6 & 3.8 & 4.5 & 8.1 \\
\hline & & & $\mathrm{C}_{2} \mathrm{H}_{5}$ & 3.9 & 2.7 & 3.3 & 3.4 & 3.8 & 4.4 & 8.3 \\
\hline & & \multirow[t]{2}{*}{$G / \mathrm{MCH}$} & $\mathrm{C}_{6} \mathrm{H}_{13}$ & 4.1 & 2.1 & 2.7 & 2.7 & 3.1 & 2.4 & 6.8 \\
\hline & & & $\mathrm{C}_{2} \mathrm{H}_{5}$ & 4.1 & 2.3 & 2.7 & 2.7 & 3.0 & 3.7 & 7.0 \\
\hline & & \multirow{2}{*}{$G$ / THF } & $\mathrm{C}_{6} \mathrm{H}_{13}$ & 3.9 & 3.2 & 3.5 & 3.8 & 3.8 & 4.6 & 8.1 \\
\hline & & & $\mathrm{C}_{2} \mathrm{H}_{5}$ & 4.3 & 3.1 & 3.5 & 3.7 & 4.1 & 4.6 & 8.8 \\
\hline \multirow[t]{12}{*}{ NHxInd } & \multirow{12}{*}{$\begin{array}{l}\text { reverse } \\
\text { ESPT }\end{array}$} & \multirow[t]{2}{*}{$E / \mathrm{MCH}$} & $\mathrm{C}_{6} \mathrm{H}_{13}$ & 3.3 & 2.7 & 3.5 & 4.3 & 3.1 & 3.3 & 12.1 \\
\hline & & & $\mathrm{C}_{2} \mathrm{H}_{5}$ & 3.2 & 2.6 & 3.6 & 4.3 & 3.1 & 3.3 & 12.2 \\
\hline & & \multirow[t]{2}{*}{$E / \mathrm{THF}$} & $\mathrm{C}_{6} \mathrm{H}_{13}$ & 3.0 & 2.2 & 2.8 & 3.7 & 2.4 & 2.6 & 10.5 \\
\hline & & & $\mathrm{C}_{2} \mathrm{H}_{5}$ & 3.0 & 2.2 & 2.8 & 3.7 & 2.4 & 2.6 & 10.6 \\
\hline & & \multirow[t]{2}{*}{$E_{0} / \mathrm{MCH}$} & $\mathrm{C}_{6} \mathrm{H}_{13}$ & 0.3 & -0.3 & 0.8 & 1.3 & 0.3 & 0.4 & 8.8 \\
\hline & & & $\mathrm{C}_{2} \mathrm{H}_{5}$ & 0.4 & -0.3 & 0.8 & 1.3 & 0.3 & 0.3 & 8.8 \\
\hline & & \multirow[t]{2}{*}{$E_{0} / \mathrm{THF}$} & $\mathrm{C}_{6} \mathrm{H}_{13}$ & -0.1 & -0.6 & 0.3 & 0.9 & -0.2 & -0.2 & 7.4 \\
\hline & & & $\mathrm{C}_{2} \mathrm{H}_{5}$ & 0.0 & -0.5 & 0.3 & 0.9 & -0.2 & -0.1 & 7.4 \\
\hline & & \multirow[t]{2}{*}{$G / \mathrm{MCH}$} & $\mathrm{C}_{6} \mathrm{H}_{13}$ & 0.2 & -0.4 & 1.0 & 1.4 & 0.3 & 0.6 & 9.0 \\
\hline & & & $\mathrm{C}_{2} \mathrm{H}_{5}$ & 0.5 & -0.4 & 0.8 & 1.2 & 0.4 & 0.4 & 9.0 \\
\hline & & \multirow[t]{2}{*}{$G / \mathrm{THF}$} & $\mathrm{C}_{6} \mathrm{H}_{13}$ & -0.1 & -0.6 & 0.4 & 1.0 & -0.2 & -0.2 & 7.8 \\
\hline & & & $\mathrm{C}_{2} \mathrm{H}_{5}$ & 0.3 & -0.2 & 0.3 & 0.9 & 0.1 & 0.1 & 7.8 \\
\hline \multirow[t]{12}{*}{ NHxInd } & \multirow{12}{*}{$\begin{array}{l}\text { forward } \\
\text { GSPT }\end{array}$} & \multirow[t]{2}{*}{$E / \mathrm{MCH}$} & $\mathrm{C}_{6} \mathrm{H}_{13}$ & 11.0 & 9.6 & 11.2 & 11.3 & 11.7 & 12.4 & 20.6 \\
\hline & & & $\mathrm{C}_{2} \mathrm{H}_{5}$ & 11.0 & 9.6 & 11.1 & 11.3 & 11.7 & 12.4 & 20.6 \\
\hline & & \multirow[t]{2}{*}{$E / \mathrm{THF}$} & $\mathrm{C}_{6} \mathrm{H}_{13}$ & 11.3 & 10.0 & 11.5 & 11.7 & 11.9 & 12.7 & 20.9 \\
\hline & & & $\mathrm{C}_{2} \mathrm{H}_{5}$ & 11.4 & 9.9 & 11.5 & 11.7 & 11.9 & 12.6 & 20.9 \\
\hline & & $E_{0} / \mathrm{MCH}$ & $\mathrm{C}_{6} \mathrm{H}_{13}$ & 8.2 & 6.9 & 8.5 & 8.6 & 9.0 & 9.6 & 17.6 \\
\hline & & & $\mathrm{C}_{2} \mathrm{H}_{5}$ & 8.2 & 6.9 & 8.4 & 8.5 & 8.9 & 9.6 & 17.6 \\
\hline & & $E_{0} / \mathrm{THF}$ & $\mathrm{C}_{6} \mathrm{H}_{13}$ & 8.6 & 7.3 & 8.9 & 9.0 & 9.3 & 10.0 & 18.0 \\
\hline & & & $\mathrm{C}_{2} \mathrm{H}_{5}$ & 8.7 & 7.3 & 8.7 & 8.9 & 9.3 & 9.9 & 18.0 \\
\hline & & $G / \mathrm{MCH}$ & $\mathrm{C}_{6} \mathrm{H}_{13}$ & 8.3 & 7.0 & 8.8 & 8.7 & 9.3 & 9.6 & 17.5 \\
\hline & & & $\mathrm{C}_{2} \mathrm{H}_{5}$ & 8.2 & 7.2 & 8.6 & 8.4 & 9.1 & 10.0 & 17.5 \\
\hline & & $G / \mathrm{THF}$ & $\mathrm{C}_{6} \mathrm{H}_{13}$ & 8.9 & 7.5 & 9.0 & 9.0 & 9.7 & 10.3 & 18.2 \\
\hline & & & $\mathrm{C}_{2} \mathrm{H}_{5}$ & 9.2 & 6.3 & 8.8 & 8.9 & 9.5 & 10.0 & 18.2 \\
\hline
\end{tabular}




\begin{tabular}{|c|c|c|c|c|c|c|c|c|c|c|}
\hline \multicolumn{11}{|c|}{ Table S7. Continued. ${ }^{a}$} \\
\hline \multirow[b]{2}{*}{ molecule } & \multirow[b]{2}{*}{ reaction } & \multirow{2}{*}{$\begin{array}{l}\text { energy }^{b} / \\
\text { medium }^{c}\end{array}$} & \multirow{2}{*}{$\begin{array}{l}N \text {-hexyl } \\
\text { model }\end{array}$} & \multirow[b]{2}{*}{ B3LYP } & \multirow[b]{2}{*}{ PBE0 } & \multicolumn{3}{|c|}{ method } & \multirow[b]{2}{*}{$\omega B 97 X-D$} & \multirow[b]{2}{*}{$\mathrm{HF} / \mathrm{CIS}$} \\
\hline & & & & & & M06-2X & MN15 & $\mathrm{CAM}^{d}$ & & \\
\hline \multirow[t]{12}{*}{ NHxInd } & reverse & $E / \mathrm{MCH}$ & $\mathrm{C}_{6} \mathrm{H}_{13}$ & 1.5 & 1.0 & 3.3 & 3.1 & 2.2 & 2.5 & 10.0 \\
\hline & GSPT & & $\mathrm{C}_{2} \mathrm{H}_{5}$ & 1.5 & 0.9 & 3.2 & 3.1 & 2.2 & 2.4 & 10.0 \\
\hline & & $E / \mathrm{THF}$ & $\mathrm{C}_{6} \mathrm{H}_{13}$ & 1.4 & 0.9 & 3.3 & 3.1 & 2.2 & 2.5 & 10.2 \\
\hline & & & $\mathrm{C}_{2} \mathrm{H}_{5}$ & 1.4 & 0.9 & 3.3 & 3.1 & 2.2 & 2.4 & 10.3 \\
\hline & & $E_{0} / \mathrm{MCH}$ & $\mathrm{C}_{6} \mathrm{H}_{13}$ & -1.1 & -1.5 & 0.6 & 0.4 & -0.5 & -0.3 & 6.5 \\
\hline & & & $\mathrm{C}_{2} \mathrm{H}_{5}$ & -1.1 & -1.5 & 0.5 & 0.3 & -0.5 & -0.3 & 6.5 \\
\hline & & $E_{0} / \mathrm{THF}$ & $\mathrm{C}_{6} \mathrm{H}_{13}$ & -1.2 & -1.6 & 0.6 & 0.3 & -0.6 & -0.3 & 6.8 \\
\hline & & & $\mathrm{C}_{2} \mathrm{H}_{5}$ & -1.1 & -1.5 & 0.5 & 0.3 & -0.5 & -0.3 & 6.7 \\
\hline & & $G / \mathrm{MCH}$ & $\mathrm{C}_{6} \mathrm{H}_{13}$ & -1.2 & -1.5 & 0.8 & 0.4 & -0.5 & -0.2 & 6.2 \\
\hline & & & $\mathrm{C}_{2} \mathrm{H}_{5}$ & -1.0 & -1.1 & 0.6 & 0.1 & -0.5 & -0.3 & 6.2 \\
\hline & & $G / \mathrm{THF}$ & $\mathrm{C}_{6} \mathrm{H}_{13}$ & -1.1 & -1.5 & 0.5 & 0.3 & -0.6 & 0.1 & 7.0 \\
\hline & & & $\mathrm{C}_{2} \mathrm{H}_{5}$ & -0.6 & -0.9 & 0.6 & 0.5 & -0.2 & -0.1 & 6.6 \\
\hline $\begin{array}{l}{ }^{a} \text { All calcu } \\
\text { ZPVE cor } \\
\text { B3LYP. }\end{array}$ & $\begin{array}{l}\text { ns carrie } \\
\text { ions }\left(E_{0}\right) \text {, }\end{array}$ & $\begin{array}{l}\text { ti with the } \\
\text { Ifree eners }\end{array}$ & $\begin{array}{l}-311 \mathrm{G}(\mathrm{d} \\
\text { les }(G) .{ }^{9}\end{array}$ & $\begin{array}{l}\text { asis set. } \\
{[\text { and } \mathrm{TI}}\end{array}$ & $\begin{array}{l}\text { riers g } \\
\text { enote } 1\end{array}$ & $\begin{array}{l}\text { in terms } \\
\text { lcycloh }\end{array}$ & electr & $\begin{array}{l}\text { nergies } \\
\text { hydrof }\end{array}$ & $\begin{array}{l}\text { electronic e } \\
\text { respectivel }\end{array}$ & $\begin{array}{l}\text { gies plus } \\
\text { CAM- }\end{array}$ \\
\hline
\end{tabular}




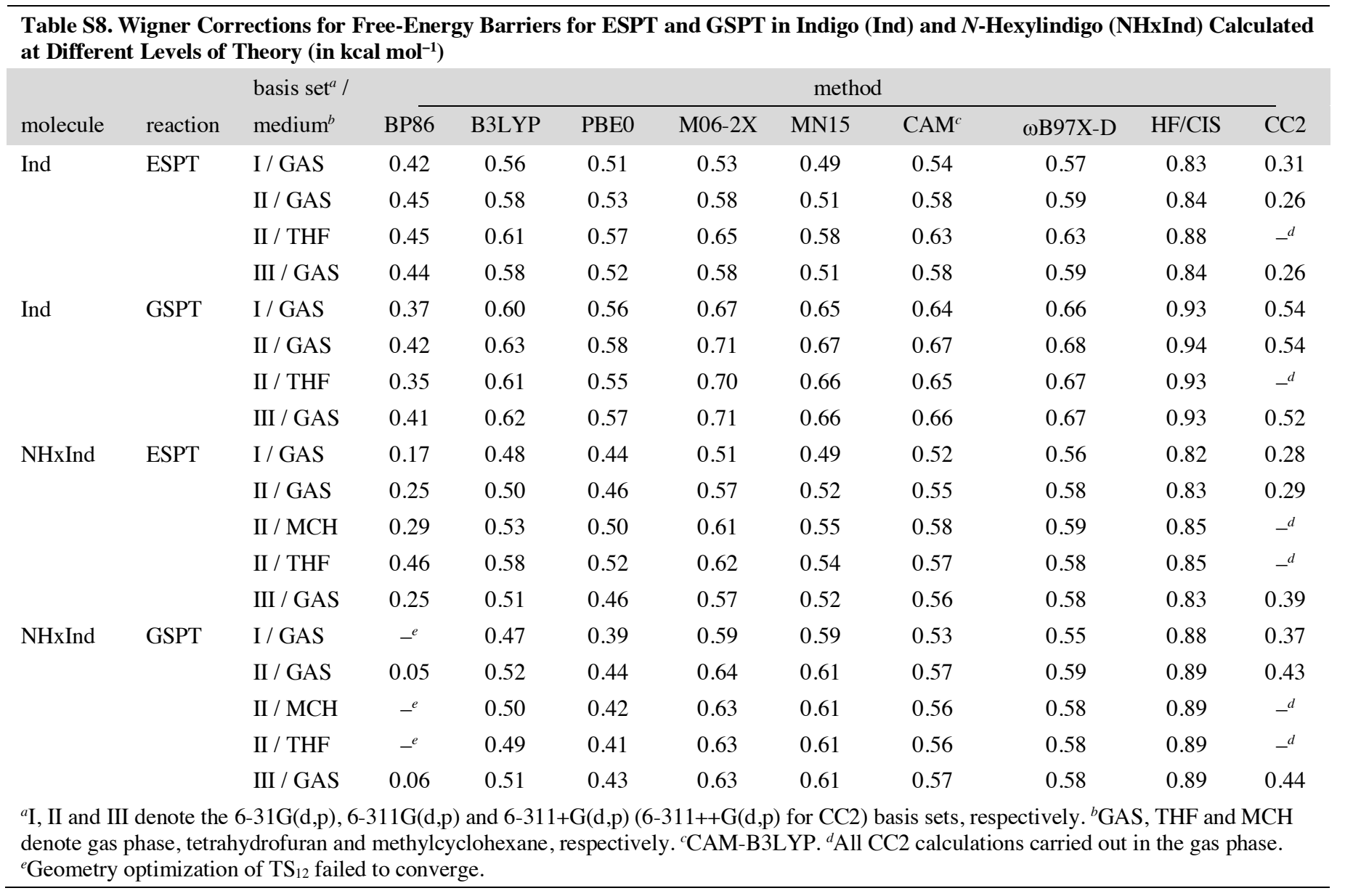




\begin{tabular}{|c|c|c|c|c|c|c|c|c|c|c|}
\hline \multicolumn{11}{|c|}{$\begin{array}{l}\text { Table S9. Relevant Interatomic Distances in Stationary Points for ESPT and GSPT in Indigo (Ind) in the Gas Phase Calculated with } \\
\text { Different Methods and Mean Absolute Errors (MAEs) for the Methods Relative to the Corresponding CC2 Values (in } \AA)^{a}\end{array}$} \\
\hline reaction & $\begin{array}{l}\text { isomer / } \\
\text { bond }^{b}\end{array}$ & \multicolumn{9}{|c|}{ method } \\
\hline \multirow[t]{15}{*}{ ESPT } & $1 / \mathrm{N} 1-\mathrm{H}$ & 1.031 & 1.022 & 1.016 & 1.017 & 1.020 & 1.017 & 1.016 & 1.000 & 1.025 \\
\hline & $1 / \mathrm{N} 1-\mathrm{C} 2$ & 1.428 & 1.376 & 1.375 & 1.369 & 1.375 & 1.369 & 1.368 & 1.353 & 1.371 \\
\hline & $1 / \mathrm{C} 4-\mathrm{O} 5$ & 1.253 & 1.244 & 1.236 & 1.231 & 1.238 & 1.234 & 1.233 & 1.212 & 1.260 \\
\hline & $1 / \mathrm{O} 5-\mathrm{H}$ & 2.090 & 2.055 & 2.229 & 2.239 & 2.231 & 2.225 & 2.237 & 2.275 & 2.198 \\
\hline & $1 / \mathrm{N} 1-\mathrm{O} 5$ & 2.847 & 2.808 & 2.921 & 2.922 & 2.921 & 2.915 & 2.923 & 2.928 & 2.918 \\
\hline & $\mathrm{TS}_{12} / \mathrm{N} 1-\mathrm{H}$ & 1.318 & 1.279 & 1.256 & 1.222 & 1.235 & 1.241 & 1.243 & 1.204 & 1.192 \\
\hline & $\mathrm{TS}_{12} / \mathrm{C} 4-\mathrm{O} 5$ & 1.297 & 1.284 & 1.276 & 1.271 & 1.277 & 1.275 & 1.275 & 1.261 & 1.291 \\
\hline & $\mathrm{TS}_{12} / \mathrm{O} 5-\mathrm{H}$ & 1.220 & 1.240 & 1.248 & 1.301 & 1.281 & 1.275 & 1.268 & 1.289 & 1.353 \\
\hline & $\mathrm{TS}_{12} / \mathrm{N} 1-\mathrm{O} 5$ & 2.468 & 2.445 & 2.432 & 2.442 & 2.439 & 2.437 & 2.434 & 2.411 & 2.470 \\
\hline & 2 / N1-H & 1.842 & 1.932 & 1.892 & 1.973 & 1.950 & 1.926 & 1.940 & 2.093 & 1.785 \\
\hline & 2 / N1-C2 & 1.401 & 1.390 & 1.380 & 1.378 & 1.381 & 1.380 & 1.379 & 1.365 & 1.426 \\
\hline & $2 / \mathrm{C} 2-\mathrm{C} 3$ & 1.397 & 1.392 & 1.389 & 1.392 & 1.393 & 1.388 & 1.391 & 1.393 & 1.386 \\
\hline & 2 / C3-C4 & 1.426 & 1.415 & 1.411 & 1.411 & 1.410 & 1.407 & 1.408 & 1.396 & 1.434 \\
\hline & $2 / \mathrm{C} 4-\mathrm{O} 5$ & 1.334 & 1.329 & 1.320 & 1.324 & 1.325 & 1.323 & 1.322 & 1.319 & 1.324 \\
\hline & $2 / \mathrm{O} 5-\mathrm{H}$ & 1.011 & 0.988 & 0.987 & 0.981 & 0.986 & 0.985 & 0.982 & 0.951 & 1.007 \\
\hline \multirow{11}{*}{ GSPT } & $1 / \mathrm{C} 3-\mathrm{C} 4$ & 1.495 & 1.493 & 1.486 & 1.494 & 1.489 & 1.489 & 1.492 & 1.500 & 1.486 \\
\hline & $1 / \mathrm{C} 4-\mathrm{O} 5$ & 1.241 & 1.226 & 1.222 & 1.215 & 1.223 & 1.218 & 1.218 & 1.194 & 1.244 \\
\hline & $1 / \mathrm{O} 5-\mathrm{H}$ & 2.259 & 2.279 & 2.259 & 2.280 & 2.269 & 2.271 & 2.281 & 2.330 & 2.256 \\
\hline & 1 / N1-O5 & 2.922 & 2.918 & 2.898 & 2.902 & 2.899 & 2.900 & 2.907 & 2.922 & 2.910 \\
\hline & $\mathrm{TS}_{12} / \mathrm{N} 1-\mathrm{H}$ & 1.337 & 1.306 & 1.296 & 1.276 & 1.284 & 1.295 & 1.297 & 1.273 & 1.276 \\
\hline & $\mathrm{TS}_{12} / \mathrm{N} 1-\mathrm{C} 2$ & 1.352 & 1.343 & 1.337 & 1.337 & 1.339 & 1.335 & 1.335 & 1.328 & 1.351 \\
\hline & $\mathrm{TS}_{12} / \mathrm{C} 2-\mathrm{C} 3$ & 1.391 & 1.380 & 1.377 & 1.374 & 1.376 & 1.372 & 1.374 & 1.362 & 1.388 \\
\hline & $\mathrm{TS}_{12} / \mathrm{C} 3-\mathrm{C} 4$ & 1.442 & 1.434 & 1.429 & 1.430 & 1.428 & 1.424 & 1.425 & 1.421 & 1.438 \\
\hline & $\mathrm{TS}_{12} / \mathrm{C} 4-\mathrm{O} 5$ & 1.302 & 1.288 & 1.281 & 1.277 & 1.281 & 1.280 & 1.280 & 1.259 & 1.299 \\
\hline & $\mathrm{TS}_{12} / \mathrm{O} 5-\mathrm{H}$ & 1.189 & 1.200 & 1.193 & 1.217 & 1.210 & 1.199 & 1.192 & 1.196 & 1.221 \\
\hline & $\mathrm{TS}_{12} / \mathrm{N} 1-\mathrm{O} 5$ & 2.452 & 2.427 & 2.413 & 2.412 & 2.415 & 2.414 & 2.411 & 2.386 & 2.427 \\
\hline
\end{tabular}




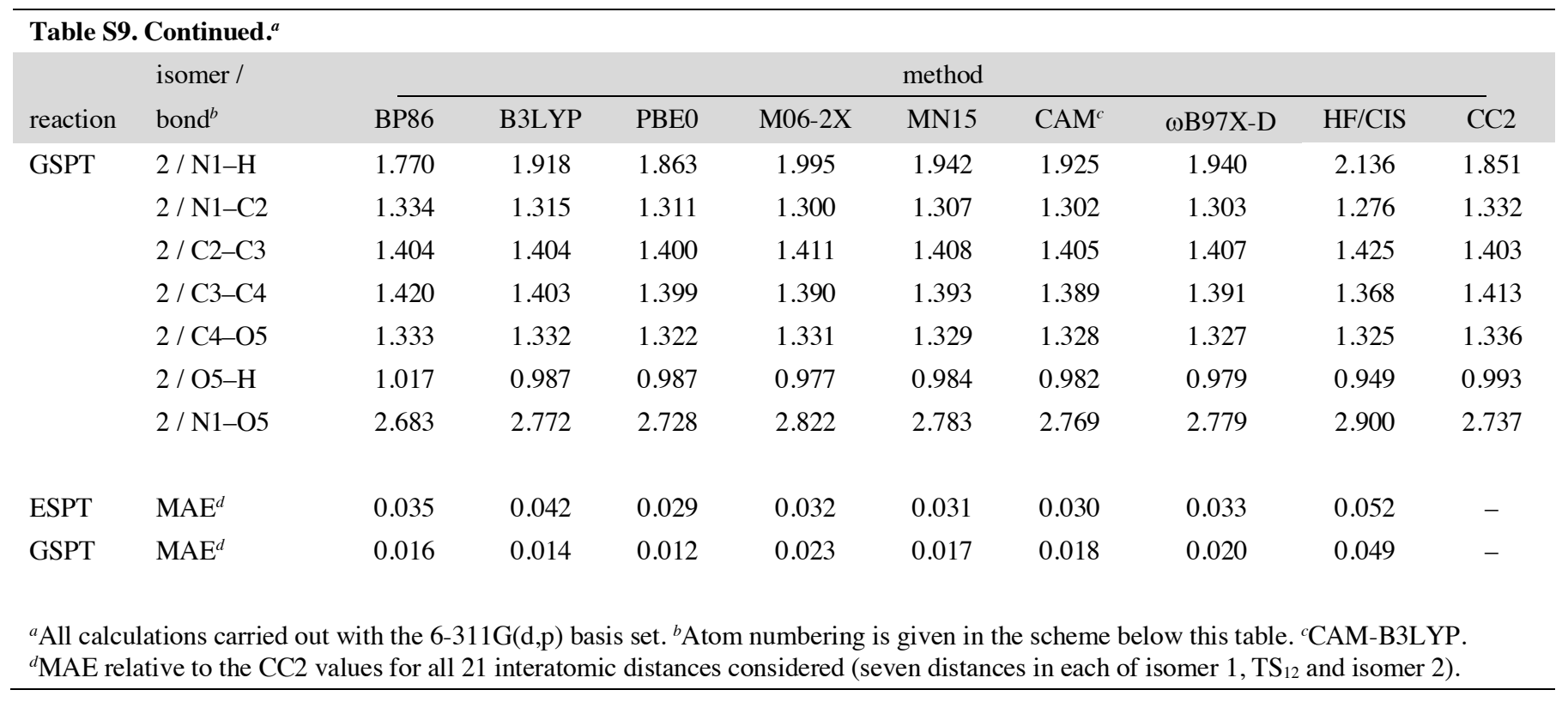

Atom numbering for Ind used in Table S9:<smiles>O=C1C(=C2Nc3[nH]c4ccccc4c3N2)C(=O)c2ccccc21</smiles>

Isomer 1

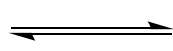<smiles>O=C1C(c2[nH]c3ccccc3c2O)=Nc2ccccc21</smiles>

Isomer 2 


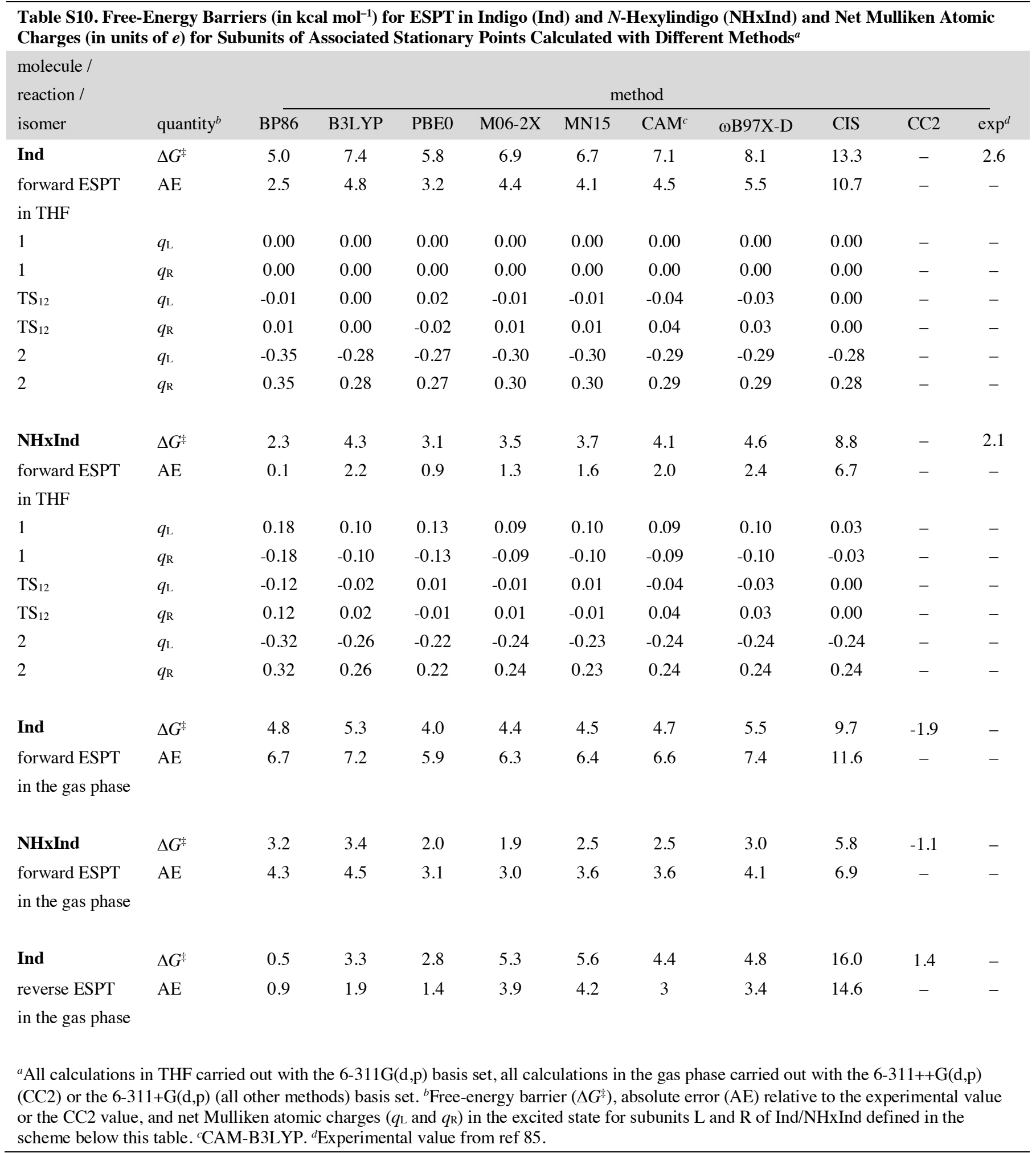


Subunits L and R of Ind/NHxInd to which net Mulliken atomic charges are assigned in Table S10:

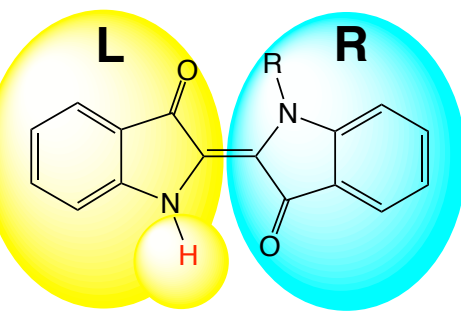

Isomer 1

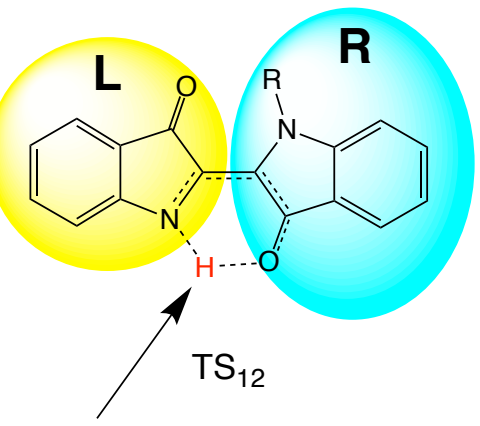

In $\mathrm{TS}_{12}$, the charge of the transferred proton is divided equally between subunits $L$ and $R$

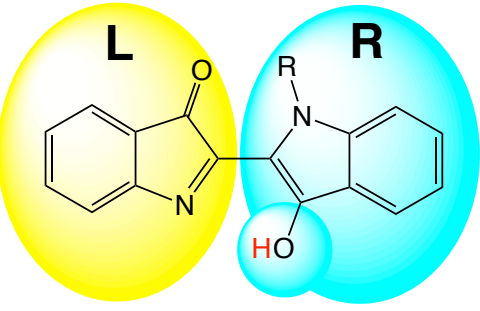

Isomer 2 


\begin{tabular}{|c|c|c|c|c|c|c|c|c|c|}
\hline \multicolumn{10}{|c|}{$\begin{array}{l}\text { Table S11. Net Mulliken Atomic Charges in Subunits } \\
\text { Different Methods in Different Media (in units of } e)^{a}\end{array}$} \\
\hline \multirow{2}{*}{$\begin{array}{l}\text { medium }^{b} / \\
\text { isomer }\end{array}$} & \multirow[b]{2}{*}{ quantity $^{c}$} & \multicolumn{8}{|c|}{ method } \\
\hline & & BP86 & B3LYP & PBE0 & M06-2X & MN15 & $\mathrm{CAM}^{d}$ & $\omega B 97 X-D$ & $\overline{\mathrm{CIS}}$ \\
\hline GAS / 1 & $q_{\mathrm{L}}$ & 0.19 & 0.20 & 0.23 & 0.12 & 0.14 & 0.12 & 0.12 & 0.09 \\
\hline GAS / 1 & $q_{\mathrm{R}}$ & -0.19 & -0.20 & -0.23 & -0.12 & -0.14 & -0.12 & -0.12 & -0.09 \\
\hline GAS / $\mathrm{TS}_{12}$ & $q_{\mathrm{L}}$ & 0.01 & 0.02 & 0.07 & 0.06 & 0.07 & 0.03 & 0.04 & 0.09 \\
\hline GAS / $\mathrm{TS}_{12}$ & $q_{\mathrm{R}}$ & -0.01 & -0.02 & -0.07 & -0.06 & -0.07 & -0.03 & -0.04 & -0.09 \\
\hline GAS / 2 & $q_{\mathrm{L}}$ & -0.30 & -0.26 & -0.20 & -0.17 & -0.17 & -0.18 & -0.17 & -0.17 \\
\hline GAS / 2 & $q_{\mathrm{R}}$ & 0.30 & 0.26 & 0.20 & 0.17 & 0.17 & 0.18 & 0.17 & 0.17 \\
\hline $\mathrm{MCH} / 1$ & $q_{\mathrm{L}}$ & 0.20 & 0.17 & 0.15 & 0.11 & 0.13 & 0.10 & 0.11 & 0.07 \\
\hline $\mathrm{MCH} / 1$ & $q_{\mathrm{R}}$ & -0.20 & -0.17 & -0.15 & -0.11 & -0.13 & -0.10 & -0.11 & -0.07 \\
\hline $\mathrm{MCH} / \mathrm{TS}_{12}$ & $q_{\mathrm{L}}$ & 0.01 & 0.02 & 0.06 & 0.03 & 0.05 & 0.00 & 0.01 & 0.05 \\
\hline $\mathrm{MCH} / \mathrm{TS}_{12}$ & $q_{\mathrm{R}}$ & -0.01 & -0.02 & -0.06 & -0.03 & -0.05 & 0.00 & -0.01 & -0.05 \\
\hline $\mathrm{MCH} / 2$ & $q_{\mathrm{L}}$ & -0.32 & -0.24 & -0.19 & -0.20 & -0.19 & -0.20 & -0.20 & -0.19 \\
\hline $\mathrm{MCH} / 2$ & $q_{\mathrm{R}}$ & 0.32 & 0.24 & 0.19 & 0.20 & 0.19 & 0.20 & 0.20 & 0.19 \\
\hline THF / 1 & $q_{\mathrm{L}}$ & 0.18 & 0.10 & 0.13 & 0.09 & 0.10 & 0.09 & 0.10 & 0.03 \\
\hline THF / 1 & $q_{\mathrm{R}}$ & -0.18 & -0.10 & -0.13 & -0.09 & -0.10 & -0.09 & -0.10 & -0.03 \\
\hline $\mathrm{THF} / \mathrm{TS}_{12}$ & $q_{\mathrm{L}}$ & -0.12 & -0.02 & 0.01 & -0.01 & 0.01 & -0.04 & -0.03 & 0.00 \\
\hline $\mathrm{THF} / \mathrm{TS}_{12}$ & $q_{\mathrm{R}}$ & 0.12 & 0.02 & -0.01 & 0.01 & -0.01 & 0.04 & 0.03 & 0.00 \\
\hline THF / 2 & $q_{\mathrm{L}}$ & -0.32 & -0.26 & -0.22 & -0.24 & -0.23 & -0.24 & -0.24 & -0.24 \\
\hline THF / 2 & $q_{\mathrm{R}}$ & 0.32 & 0.26 & 0.22 & 0.24 & 0.23 & 0.24 & 0.24 & 0.24 \\
\hline
\end{tabular}

Subunits L and R of NHxInd to which net Mulliken atomic charges are assigned in Table S11:

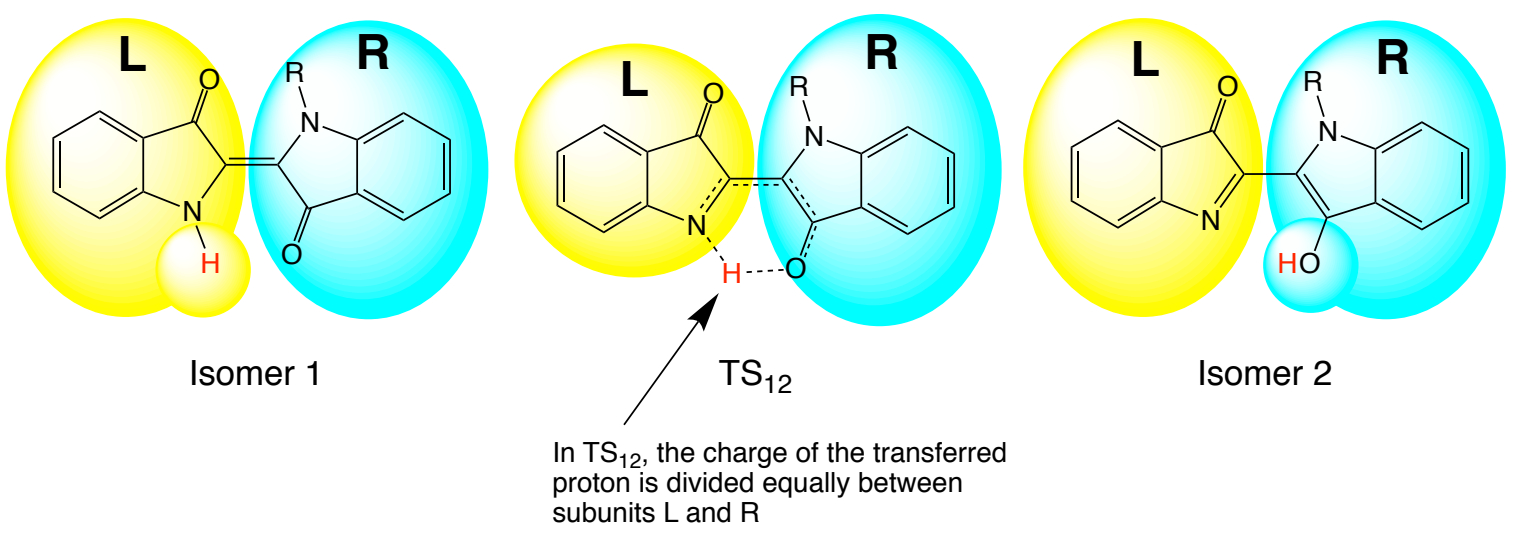

Comments on the results in Table S11: With one exception, all density functionals predict $\mathrm{TS}_{12}$ for ESPT in NHxInd to have a non-polar excited-state charge distribution with respect to the two 
molecular subunits in each of the three media (gas phase, $\mathrm{MCH}$ and THF) considered. This exception is the prediction by BP86 that $\mathrm{TS}_{12}$ has a somewhat polar charge distribution in THF. Through this prediction, BP86 appears likely to yield a smaller free-energy barrier than the other functionals for forward ESPT in NHxInd in THF, simply because such a charge distribution would allow $\mathrm{TS}_{12}$ to be better stabilized by the polar THF solvent. This might explain why BP86 is the only functional (see Figure 4b) that does not reproduce the experimental finding that the forward ESPT barrier for NHxInd is slightly $\left(1.1 \mathrm{kcal} \mathrm{mol}^{-1}\right)$ larger in THF than in MCH. 


\begin{tabular}{|c|c|c|c|c|c|c|c|c|c|c|}
\hline \multirow{2}{*}{$\begin{array}{l}\text { molecule / } \\
\text { reaction }\end{array}$} & \multirow{2}{*}{$\begin{array}{l}\text { isomer / } \\
\text { bond }\end{array}$} & \multirow[b]{2}{*}{ medium } & \multirow[b]{2}{*}{ BP86 } & \multirow[b]{2}{*}{ B3LYP } & \multirow[b]{2}{*}{ PBE0 } & \multicolumn{2}{|c|}{ method } & \multirow[b]{2}{*}{$\mathrm{CAM}^{b}$} & \multirow[b]{2}{*}{$\omega B 97 X-D$} & \multirow[b]{2}{*}{$\mathrm{HF} / \mathrm{CIS}$} \\
\hline & & & & & & M06-2X & MN15 & & & \\
\hline Ind & $1 / \mathrm{N}-\mathrm{H}$ & THF & 1.029 & 1.016 & 1.016 & 1.017 & 1.019 & 1.017 & 1.015 & 1.000 \\
\hline \multirow[t]{8}{*}{ ESPT } & $1 / \mathrm{O}-\mathrm{H}$ & THF & 2.169 & 2.292 & 2.273 & 2.280 & 2.273 & 2.267 & 2.281 & 2.312 \\
\hline & $1 / \mathrm{N}-\mathrm{O}$ & THF & 2.891 & 2.959 & 2.941 & 2.940 & 2.940 & 2.934 & 2.944 & 2.950 \\
\hline & $\mathrm{TS}_{12} / \mathrm{N}-\mathrm{H}$ & THF & 1.308 & 1.274 & 1.260 & 1.250 & 1.252 & 1.267 & 1.271 & 1.231 \\
\hline & $\mathrm{TS}_{12} / \mathrm{O}-\mathrm{H}$ & THF & 1.224 & 1.242 & 1.243 & 1.266 & 1.260 & 1.245 & 1.235 & 1.253 \\
\hline & $\mathrm{TS}_{12} / \mathrm{N}-\mathrm{O}$ & THF & 2.462 & 2.442 & 2.430 & 2.438 & 2.437 & 2.436 & 2.432 & 2.408 \\
\hline & $2 / \mathrm{N}-\mathrm{H}$ & THF & 1.884 & 1.898 & 1.846 & 1.918 & 1.901 & 1.867 & 1.880 & 2.051 \\
\hline & $2 / \mathrm{O}-\mathrm{H}$ & THF & 1.007 & 0.990 & 0.991 & 0.984 & 0.989 & 0.990 & 0.986 & 0.953 \\
\hline & $2 / \mathrm{N}-\mathrm{O}$ & $\mathrm{THF}$ & 2.768 & 2.758 & 2.716 & 2.764 & 2.754 & 2.729 & 2.737 & 2.835 \\
\hline Ind & $1 / \mathrm{N}-\mathrm{H}$ & THF & 1.021 & 1.011 & 1.010 & 1.010 & 1.013 & 1.009 & 1.008 & 0.993 \\
\hline \multirow[t]{8}{*}{ GSPT } & $1 / \mathrm{O}-\mathrm{H}$ & THF & 2.299 & 2.314 & 2.294 & 2.314 & 2.304 & 2.304 & 2.315 & 2.359 \\
\hline & $1 / \mathrm{N}-\mathrm{O}$ & THF & 2.940 & 2.933 & 2.913 & 2.915 & 2.913 & 2.914 & 2.922 & 2.933 \\
\hline & $\mathrm{TS}_{12} / \mathrm{N}-\mathrm{H}$ & THF & 1.353 & 1.311 & 1.303 & 1.277 & 1.287 & 1.298 & 1.299 & 1.273 \\
\hline & $\mathrm{TS}_{12} / \mathrm{O}-\mathrm{H}$ & THF & 1.170 & 1.189 & 1.182 & 1.211 & 1.202 & 1.192 & 1.185 & 1.191 \\
\hline & $\mathrm{TS}_{12} / \mathrm{N}-\mathrm{O}$ & THF & 2.451 & 2.424 & 2.410 & 2.408 & 2.411 & 2.411 & 2.408 & 2.383 \\
\hline & $2 / \mathrm{N}-\mathrm{H}$ & THF & 1.705 & 1.877 & 1.819 & 1.962 & 1.908 & 1.889 & 1.908 & 2.113 \\
\hline & $2 / \mathrm{O}-\mathrm{H}$ & THF & 1.025 & 0.989 & 0.991 & 0.978 & 0.986 & 0.984 & 0.981 & 0.950 \\
\hline & $2 / \mathrm{N}-\mathrm{O}$ & $\mathrm{THF}$ & 2.635 & 2.740 & 2.694 & 2.794 & 2.756 & 2.741 & 2.753 & 2.881 \\
\hline
\end{tabular}




\begin{tabular}{|c|c|c|c|c|c|c|c|c|c|c|}
\hline \multicolumn{11}{|c|}{$\begin{array}{l}\text { Table S13. Distances Between the Transferred Proton and the Associated Nitrogen }(\mathrm{N}-\mathrm{H}) \text { and Oxygen }(\mathrm{O}-\mathrm{H}) \text { Atoms in Stationary } \\
\text { Points for ESPT and GSPT in } N \text {-Hexylindigo (NHxInd) in Methylcyclohexane (MCH) and Tetrahydrofuran (THF) Calculated } \\
\text { with Different Methods (in } \AA)^{a}\end{array}$} \\
\hline \multirow{2}{*}{$\begin{array}{l}\text { molecule / } \\
\text { reaction }\end{array}$} & \multicolumn{6}{|l|}{ isomer / } & \multicolumn{4}{|c|}{ method } \\
\hline & bond & medium & BP86 & B3LYP & PBE0 & M06-2X & MN15 & $\mathrm{CAM}^{b}$ & $\omega B 97 X-D$ & $\mathrm{HF} / \mathrm{CIS}$ \\
\hline \multirow{18}{*}{$\begin{array}{l}\text { NHxInd } \\
\text { ESPT }\end{array}$} & $1 / \mathrm{N}-\mathrm{H}$ & MHC & 1.046 & 1.030 & 1.031 & 1.023 & 1.030 & 1.028 & 1.026 & 1.002 \\
\hline & & THF & 1.044 & 1.026 & 1.028 & 1.022 & 1.028 & 1.026 & 1.024 & 1.000 \\
\hline & $1 / \mathrm{O}-\mathrm{H}$ & MHC & 1.763 & 1.803 & 1.792 & 1.896 & 1.853 & 1.820 & 1.836 & 1.953 \\
\hline & & THF & 1.786 & 1.851 & 1.817 & 1.930 & 1.882 & 1.837 & 1.854 & 2.041 \\
\hline & $1 / \mathrm{N}-\mathrm{O}$ & MHC & 2.639 & 2.645 & 2.635 & 2.689 & 2.671 & 2.647 & 2.659 & 2.698 \\
\hline & & THF & 2.819 & 2.671 & 2.647 & 2.704 & 2.685 & 2.656 & 2.668 & 2.738 \\
\hline & $\mathrm{TS}_{12} / \mathrm{N}-\mathrm{H}$ & MHC & 1.349 & 1.287 & 1.270 & 1.255 & 1.257 & 1.271 & 1.276 & 1.220 \\
\hline & & THF & 1.286 & 1.286 & 1.276 & 1.269 & 1.268 & 1.286 & 1.291 & 1.234 \\
\hline & $\mathrm{TS}_{12} / \mathrm{O}-\mathrm{H}$ & MHC & 1.169 & 1.206 & 1.212 & 1.242 & 1.236 & 1.220 & 1.211 & 1.243 \\
\hline & & THF & 1.233 & 1.212 & 1.208 & 1.226 & 1.224 & 1.205 & 1.196 & 1.227 \\
\hline & $\mathrm{TS}_{12} / \mathrm{N}-\mathrm{O}$ & MHC & 2.447 & 2.421 & 2.411 & 2.421 & 2.419 & 2.416 & 2.414 & 2.388 \\
\hline & & THF & 2.447 & 2.425 & 2.414 & 2.420 & 2.420 & 2.417 & 2.415 & 2.387 \\
\hline & $2 / \mathrm{N}-\mathrm{H}$ & MHC & 1.703 & 1.740 & 1.680 & 1.713 & 1.725 & 1.679 & 1.696 & 1.845 \\
\hline & & THF & 1.676 & 1.706 & 1.653 & 1.690 & 1.711 & 1.660 & 1.677 & 1.837 \\
\hline & $2 / \mathrm{O}-\mathrm{H}$ & MHC & 1.023 & 1.000 & 1.006 & 1.000 & 1.000 & 1.005 & 1.000 & 0.957 \\
\hline & & THF & 1.027 & 1.005 & 1.010 & 1.005 & 1.003 & 1.009 & 1.003 & 0.958 \\
\hline & $2 / \mathrm{N}-\mathrm{O}$ & MHC & 2.617 & 2.617 & 2.575 & 2.593 & 2.600 & 2.569 & 2.578 & 2.647 \\
\hline & & THF & 2.599 & 2.595 & 2.558 & 2.580 & 2.592 & 2.559 & 2.568 & 2.642 \\
\hline \multirow{18}{*}{$\begin{array}{l}\text { NHxInd } \\
\text { GSPT }\end{array}$} & $1 / \mathrm{N}-\mathrm{H}$ & MHC & 1.031 & 1.015 & 1.015 & 1.011 & 1.016 & 1.013 & 1.011 & 0.993 \\
\hline & & THF & 1.029 & 1.014 & 1.015 & 1.011 & 1.015 & 1.012 & 1.011 & 0.993 \\
\hline & $1 / \mathrm{O}-\mathrm{H}$ & MHC & 1.842 & 1.893 & 1.872 & 1.980 & 1.951 & 1.913 & 1.925 & 2.025 \\
\hline & & THF & 1.867 & 1.921 & 1.887 & 2.004 & 1.976 & 1.931 & 1.944 & 2.046 \\
\hline & $1 / \mathrm{N}-\mathrm{O}$ & MHC & 2.650 & 2.660 & 2.642 & 2.699 & 2.686 & 2.660 & 2.669 & 2.710 \\
\hline & & THF & 2.662 & 2.673 & 2.648 & 2.709 & 2.697 & 2.669 & 2.679 & 2.721 \\
\hline & $\mathrm{TS}_{12} / \mathrm{N}-\mathrm{H}$ & MHC & $-^{c}$ & 1.323 & 1.320 & 1.283 & 1.294 & 1.307 & 1.308 & 1.272 \\
\hline & & THF & $-^{c}$ & 1.326 & 1.322 & 1.282 & 1.294 & 1.306 & 1.307 & 1.270 \\
\hline & $\mathrm{TS}_{12} / \mathrm{O}-\mathrm{H}$ & MHC & $-^{c}$ & 1.158 & 1.148 & 1.187 & 1.177 & 1.165 & 1.159 & 1.172 \\
\hline & & THF & $-^{c}$ & 1.156 & 1.146 & 1.187 & 1.176 & 1.164 & 1.159 & 1.172 \\
\hline & $\mathrm{TS}_{12} / \mathrm{N}-\mathrm{O}$ & MHC & $-^{c}$ & 2.406 & 2.395 & 2.392 & 2.394 & 2.393 & 2.391 & 2.364 \\
\hline & & THF & $-^{c}$ & 2.406 & 2.395 & 2.391 & 2.394 & 2.393 & 2.391 & 2.364 \\
\hline & $2 / \mathrm{N}-\mathrm{H}$ & MHC & $-^{c}$ & 1.655 & 1.602 & 1.765 & 1.730 & 1.683 & 1.705 & 1.946 \\
\hline & & THF & $-^{c}$ & 1.648 & 1.595 & 1.767 & 1.733 & 1.686 & 1.711 & 1.978 \\
\hline & $2 / \mathrm{O}-\mathrm{H}$ & MHC & $-^{c}$ & 1.009 & 1.015 & 0.988 & 0.996 & 0.999 & 0.993 & 0.951 \\
\hline & & THF & $-^{c}$ & 1.010 & 1.016 & 0.988 & 0.996 & 0.999 & 0.993 & 0.951 \\
\hline & $2 / \mathrm{N}-\mathrm{O}$ & MHC & $-^{c}$ & 2.557 & 2.520 & 2.625 & 2.603 & 2.568 & 2.581 & 2.728 \\
\hline & & THF & $-^{c}$ & 2.553 & 2.515 & 2.628 & 2.606 & 2.571 & 2.586 & 2.754 \\
\hline
\end{tabular}



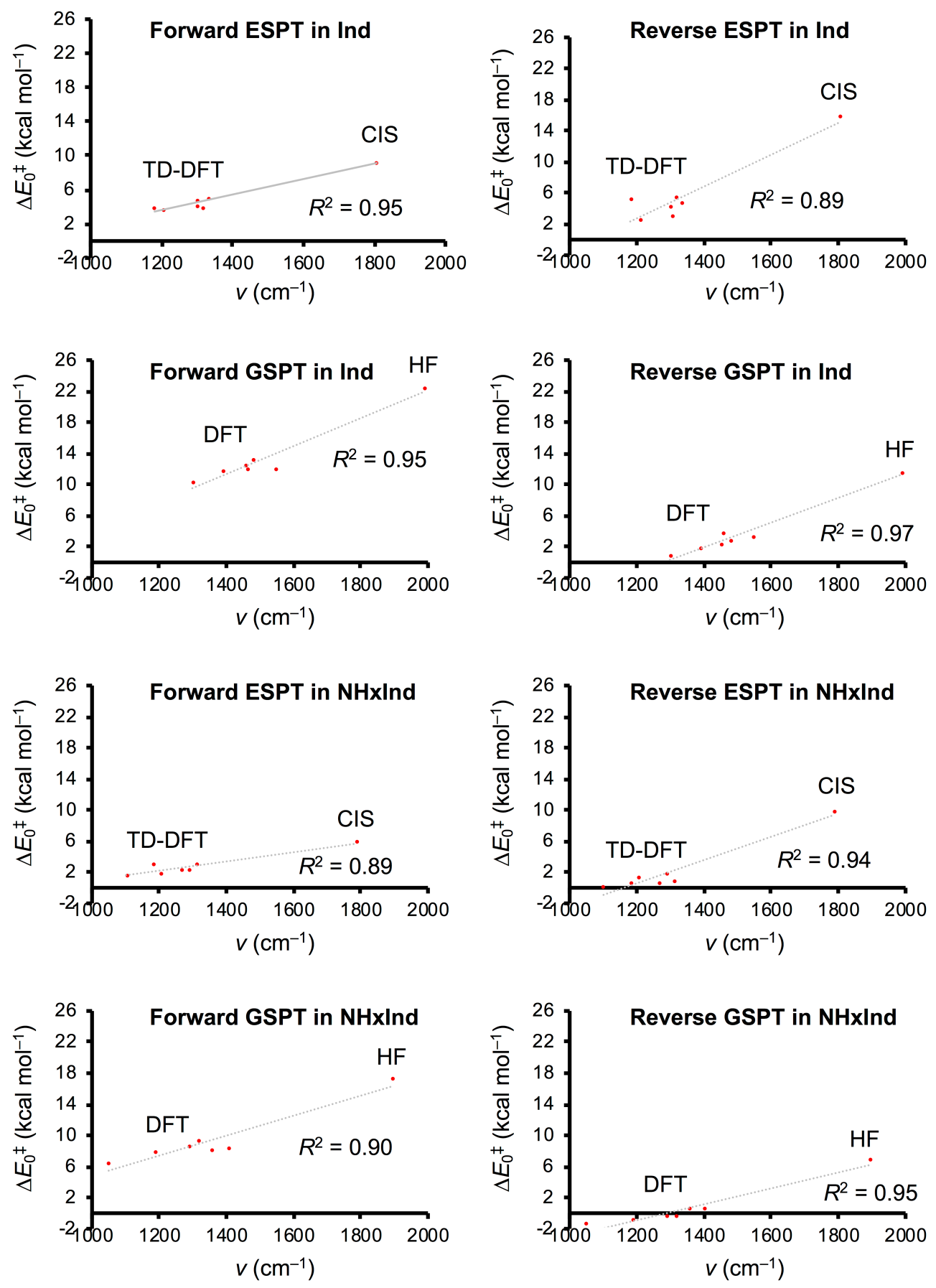

Figure S1. ZPVE-corrected energy barriers for forward and reverse ESPT and GSPT in indigo (Ind) and $N$-hexylindigo (NHxInd) in the gas phase calculated with hybrid density functionals and $\mathrm{HF} / \mathrm{CIS}$ and plotted as functions of the magnitudes of the imaginary vibrational frequency at the $\mathrm{TS}_{12}$ saddle point. All calculations carried out with the $6-311+\mathrm{G}(\mathrm{d}, \mathrm{p})$ basis set. 


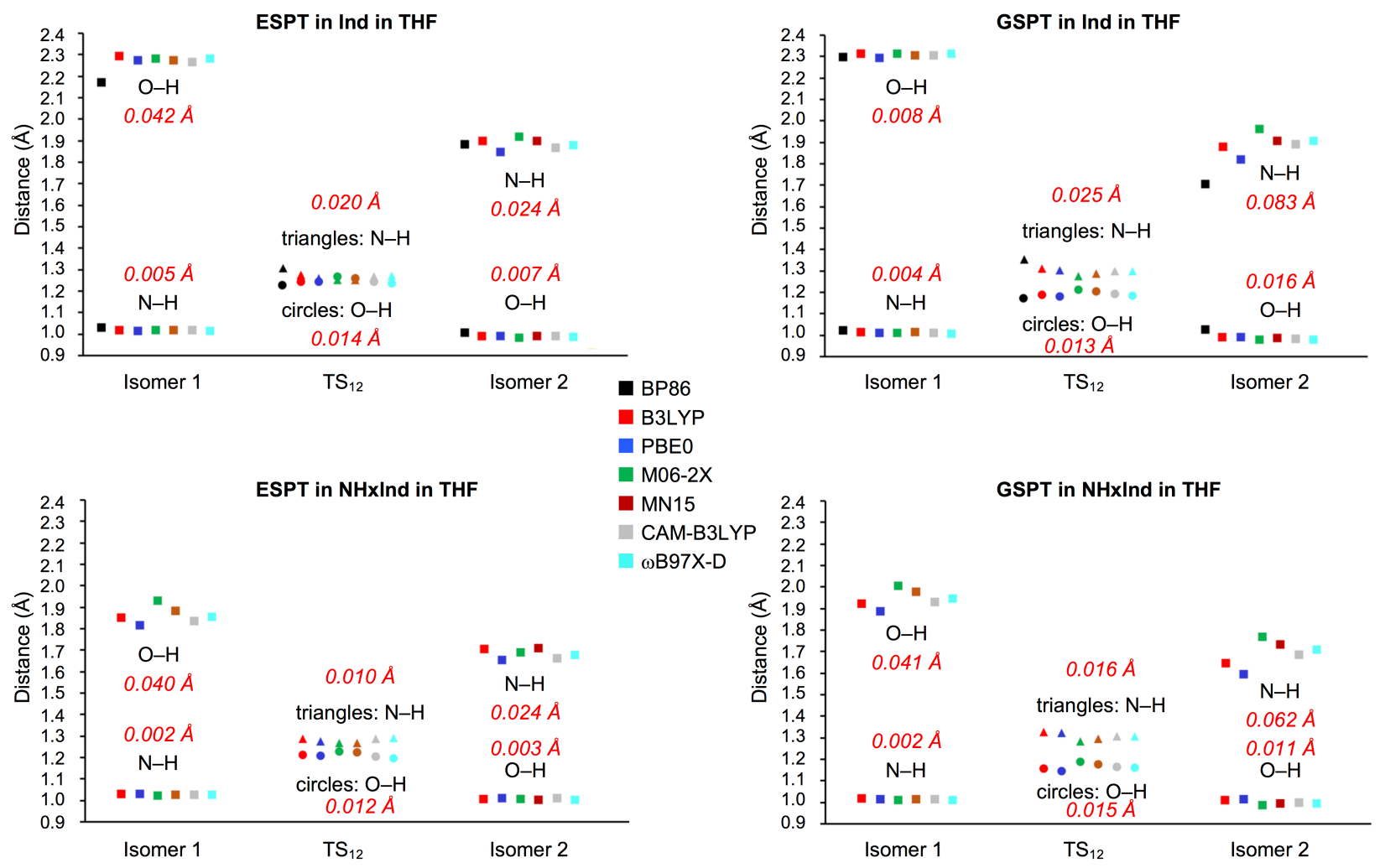

Figure S2. Distances between the transferred proton and the corresponding nitrogen $(\mathrm{N}-\mathrm{H})$ and oxygen $(\mathrm{O}-\mathrm{H})$ atoms in stationary points for ESPT and GSPT in indigo (Ind) and $N$-hexylindigo (NHxInd) in tetrahydrofuran (THF) calculated with different methods. All calculations carried out with the 6-311G(d,p) basis set. For clarity, numerical values are also given in Tables S12 and S13. Values in red and italics are standard deviations for the calculated distances. BP86 values for ESPT and GSPT in NHxInd are not shown because the BP86 geometry optimization of $\mathrm{TS}_{12}$ failed to converge for the latter reaction. 


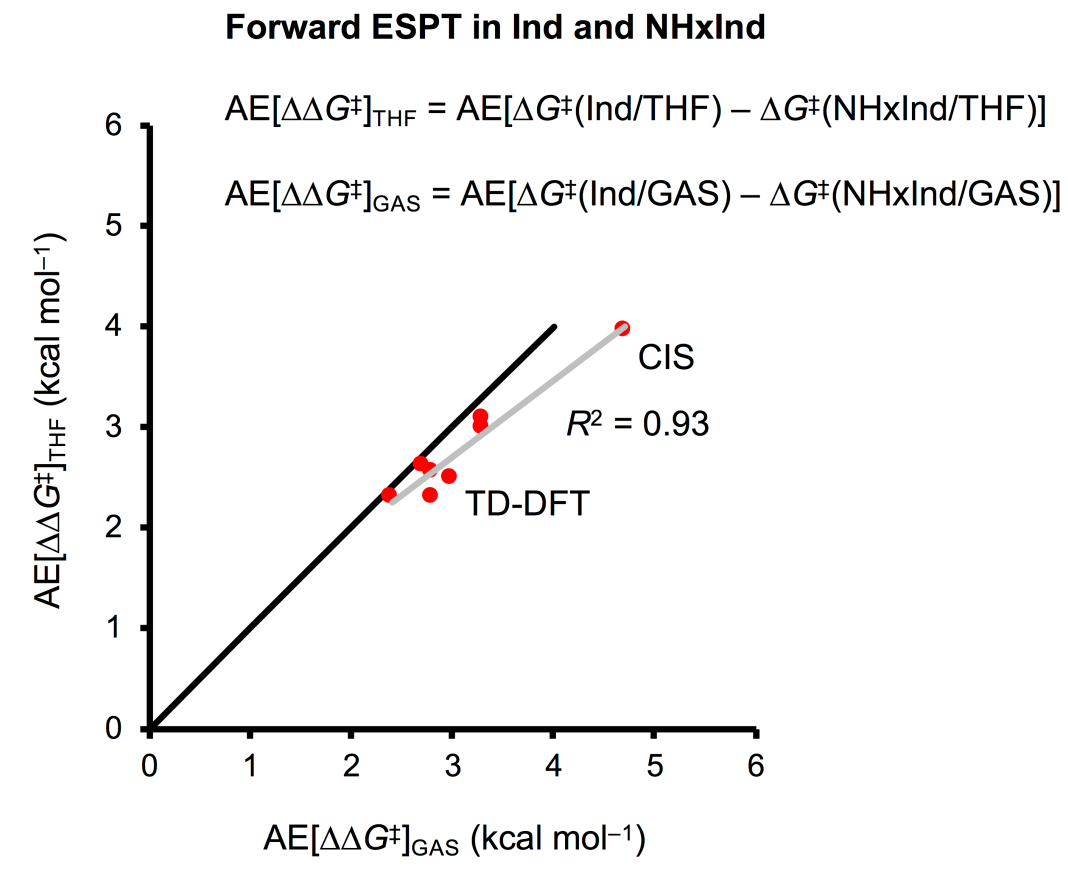

Figure S3. Absolute errors (AEs) in calculated differences in free-energy barriers for forward ESPT in indigo (Ind) and $N$-hexylindigo (NHxInd) in tetrahydrofuran (THF) plotted as function of AEs in calculated differences in free-energy barriers for the corresponding reactions in the gas phase. AEs evaluated relative to experimental (in THF) or CC2 (in the gas phase) values. All calculations in THF carried out with the $6-311 \mathrm{G}(\mathrm{d}, \mathrm{p})$ basis set, all calculations in the gas phase carried out with the $6-311+\mathrm{G}(\mathrm{d}, \mathrm{p})$ basis set. 


\section{CARTESIAN COORDINATES FOR OPTIMIZED $S_{0}$ AND $S_{1}$ GEOMETRIES}

\section{B3LYP/6-311G(d,p) geometries (in ^̊)}

$\mathrm{S}_{0}$ Ind (isomer 1), gas phase

$\begin{array}{crrr}\mathrm{C} & -2.917520 & 0.671137 & 0.000076 \\ \mathrm{C} & -4.160321 & 1.300287 & -0.000446 \\ \mathrm{C} & -5.306846 & 0.513754 & -0.000732 \\ \mathrm{C} & -5.199925 & -0.885234 & -0.000533 \\ \mathrm{C} & -3.966088 & -1.532859 & -0.000064 \\ \mathrm{C} & -2.821877 & -0.738062 & 0.000231 \\ \mathrm{H} & -4.215565 & 2.382857 & -0.000549 \\ \mathrm{H} & -6.286216 & 0.976341 & -0.001099 \\ \mathrm{H} & -6.104149 & -1.483868 & -0.000784 \\ \mathrm{H} & -3.901964 & -2.614541 & 0.000030 \\ \mathrm{C} & -1.551822 & 1.210530 & 0.000625 \\ \mathrm{~N} & -1.487673 & -1.118611 & 0.000597 \\ \mathrm{C} & -0.679135 & -0.001145 & 0.000570 \\ \mathrm{C} & 0.679104 & 0.001158 & 0.000446 \\ \mathrm{H} & -1.108981 & -2.055254 & 0.000457 \\ \mathrm{O} & -1.148519 & 2.368572 & 0.000176 \\ \mathrm{C} & 1.551805 & -1.210517 & -0.000063 \\ \mathrm{~N} & 1.487632 & 1.118629 & 0.000498 \\ \mathrm{H} & 1.108908 & 2.055262 & -0.000049 \\ \mathrm{C} & 2.821839 & 0.738095 & -0.000047 \\ \mathrm{C} & 2.917499 & -0.671102 & -0.000196 \\ \mathrm{O} & 1.148516 & -2.368556 & 0.000127 \\ \mathrm{C} & 3.966042 & 1.532902 & -0.000247 \\ \mathrm{C} & 4.160304 & -1.300240 & -0.000471 \\ \mathrm{C} & 5.199887 & 0.885291 & -0.000588 \\ \mathrm{C} & 5.306823 & -0.513694 & -0.000697 \\ \mathrm{H} & 3.901908 & 2.614584 & -0.000128 \\ \mathrm{H} & 6.104102 & 1.483937 & -0.000761 \\ \mathrm{H} & 6.286197 & -0.976272 & -0.000961 \\ \mathrm{H} & 4.215559 & -2.382810 & -0.000581\end{array}$


$\mathrm{S}_{1}$ Ind (isomer 1), gas phase

$\begin{array}{rrrr}\mathrm{C} & -2.930967 & 0.713144 & -0.000176 \\ \mathrm{C} & -4.191594 & 1.258412 & -0.000301 \\ \mathrm{C} & -5.302266 & 0.396283 & -0.000307 \\ \mathrm{C} & -5.150846 & -1.015407 & -0.000182 \\ \mathrm{C} & -3.906597 & -1.597333 & -0.000056 \\ \mathrm{C} & -2.776857 & -0.726980 & -0.000056 \\ \mathrm{H} & -4.317456 & 2.334621 & -0.000385 \\ \mathrm{H} & -6.301973 & 0.813886 & -0.000404 \\ \mathrm{H} & -6.036401 & -1.638862 & -0.000189 \\ \mathrm{H} & -3.775499 & -2.672339 & 0.000034 \\ \mathrm{C} & -1.564547 & 1.325790 & -0.000114 \\ \mathrm{~N} & -1.486236 & -1.016141 & 0.000029 \\ \mathrm{C} & -0.699713 & 0.202460 & -0.000033 \\ \mathrm{C} & 0.673130 & 0.111812 & 0.000002 \\ \mathrm{H} & -1.004970 & -1.918269 & 0.000127 \\ \mathrm{O} & -1.271353 & 2.535402 & -0.000246 \\ \mathrm{C} & 1.475815 & -1.133158 & 0.000078 \\ \mathrm{~N} & 1.518780 & 1.185025 & 0.000033 \\ \mathrm{H} & 1.174988 & 2.136366 & 0.000040 \\ \mathrm{C} & 2.844777 & 0.750881 & -0.000061 \\ \mathrm{C} & 2.861754 & -0.664020 & -0.000134 \\ \mathrm{O} & 1.016637 & -2.289555 & -0.000159 \\ \mathrm{C} & 4.017125 & 1.491886 & -0.000146 \\ \mathrm{C} & 4.077543 & -1.347098 & -0.000343 \\ \mathrm{C} & 5.226141 & 0.788914 & -0.000325 \\ \mathrm{C} & 5.260294 & -0.611642 & -0.000428 \\ \mathrm{H} & 4.000650 & 2.575712 & -0.000099 \\ \mathrm{H} & 6.157137 & 1.344397 & -0.000400 \\ \mathrm{H} & 6.215868 & -1.122652 & -0.000567 \\ \mathrm{H} & 4.086167 & -2.430964 & -0.000399\end{array}$


$\mathrm{S}_{0}$ Ind (isomer 2), gas phase

$\begin{array}{rrrr}\mathrm{C} & -2.946293 & 0.704312 & -0.000231 \\ \mathrm{C} & -4.208192 & 1.278312 & 0.000019 \\ \mathrm{C} & -5.319006 & 0.429497 & 0.000039 \\ \mathrm{C} & -5.140961 & -0.956402 & -0.000164 \\ \mathrm{C} & -3.867042 & -1.539258 & -0.000373 \\ \mathrm{C} & -2.766744 & -0.694908 & -0.000394 \\ \mathrm{H} & -4.322116 & 2.356767 & 0.000138 \\ \mathrm{H} & -6.319949 & 0.843698 & 0.000200 \\ \mathrm{H} & -6.013344 & -1.600758 & -0.000135 \\ \mathrm{H} & -3.738915 & -2.614900 & -0.000480 \\ \mathrm{C} & -1.593007 & 1.284853 & -0.000452 \\ \mathrm{~N} & -1.402470 & -1.071731 & -0.000450 \\ \mathrm{C} & -0.700138 & 0.040207 & -0.000256 \\ \mathrm{C} & 0.704001 & 0.056547 & -0.000181 \\ \mathrm{O} & -1.207794 & 2.436652 & -0.000008 \\ \mathrm{C} & 1.513047 & -1.089960 & -0.000164 \\ \mathrm{~N} & 1.528878 & 1.179041 & -0.000119 \\ \mathrm{H} & 1.173960 & 2.122681 & -0.000384 \\ \mathrm{C} & 2.835054 & 0.767729 & -0.000167 \\ \mathrm{C} & 2.868480 & -0.660656 & -0.000172 \\ \mathrm{O} & 1.061240 & -2.342880 & -0.000118 \\ \mathrm{C} & 4.018847 & 1.514737 & -0.000181 \\ \mathrm{C} & 4.101924 & -1.336199 & -0.000166 \\ \mathrm{C} & 5.217794 & 0.821267 & -0.000192 \\ \mathrm{C} & 5.264562 & -0.591067 & -0.000180 \\ \mathrm{H} & 3.997166 & 2.598298 & -0.000183 \\ \mathrm{H} & 6.147969 & 1.378260 & -0.000206 \\ \mathrm{H} & 6.226958 & -1.088597 & -0.000180 \\ \mathrm{H} & 4.126925 & -2.419308 & -0.000154 \\ \mathrm{H} & 0.075805 & -2.293886 & -0.000072\end{array}$


$\mathrm{S}_{1}$ Ind (isomer 2), gas phase

$\begin{array}{lrrr}\mathrm{C} & -2.903687 & 0.733186 & -0.000143 \\ \mathrm{C} & -4.171322 & 1.279664 & -0.000086 \\ \mathrm{C} & -5.270541 & 0.410940 & -0.000084 \\ \mathrm{C} & -5.107954 & -0.997016 & -0.000135 \\ \mathrm{C} & -3.852832 & -1.569257 & -0.000189 \\ \mathrm{C} & -2.726670 & -0.707558 & -0.000194 \\ \mathrm{H} & -4.307998 & 2.354892 & -0.000048 \\ \mathrm{H} & -6.274377 & 0.820422 & -0.000042 \\ \mathrm{H} & -5.989142 & -1.627562 & -0.000131 \\ \mathrm{H} & -3.710145 & -2.643190 & -0.000227 \\ \mathrm{C} & -1.548336 & 1.310011 & -0.000166 \\ \mathrm{~N} & -1.430754 & -1.064256 & -0.000235 \\ \mathrm{C} & -0.711070 & 0.125183 & -0.000217 \\ \mathrm{C} & 0.680525 & 0.077496 & -0.000238 \\ \mathrm{O} & -1.168697 & 2.501144 & -0.000119 \\ \mathrm{C} & 1.487658 & -1.084952 & -0.000248 \\ \mathrm{~N} & 1.510583 & 1.187323 & -0.000235 \\ \mathrm{H} & 1.132804 & 2.126512 & -0.000147 \\ \mathrm{C} & 2.815759 & 0.771211 & -0.000206 \\ \mathrm{C} & 2.841662 & -0.658949 & -0.000223 \\ \mathrm{O} & 1.046054 & -2.338614 & -0.000278 \\ \mathrm{C} & 4.001619 & 1.510656 & -0.000180 \\ \mathrm{C} & 4.071545 & -1.342333 & -0.000217 \\ \mathrm{C} & 5.198210 & 0.808897 & -0.000170 \\ \mathrm{C} & 5.238420 & -0.602976 & -0.000189 \\ \mathrm{H} & 3.985862 & 2.594081 & -0.000167 \\ \mathrm{H} & 6.131164 & 1.361244 & -0.000148 \\ \mathrm{H} & 6.198256 & -1.105262 & -0.000184 \\ \mathrm{H} & 4.090445 & -2.425545 & -0.000234 \\ \mathrm{H} & 0.059596 & -2.293047 & -0.000287\end{array}$


$\mathrm{S}_{0}$ Ind $\left(\mathrm{TS}_{12}\right)$, gas phase

$\begin{array}{lrrr}\mathrm{C} & 2.959653 & 0.675842 & 0.000093 \\ \mathrm{C} & 4.264486 & 1.151804 & 0.000100 \\ \mathrm{C} & 5.308850 & 0.227064 & 0.000053 \\ \mathrm{C} & 5.029838 & -1.144414 & 0.000002 \\ \mathrm{C} & 3.720837 & -1.634787 & -0.000006 \\ \mathrm{C} & 2.682536 & -0.710946 & 0.000041 \\ \mathrm{H} & 4.455104 & 2.219258 & 0.000140 \\ \mathrm{H} & 6.337398 & 0.566710 & 0.000057 \\ \mathrm{H} & 5.853259 & -1.850283 & -0.000034 \\ \mathrm{H} & 3.520132 & -2.699265 & -0.000046 \\ \mathrm{C} & 1.661846 & 1.372549 & 0.000133 \\ \mathrm{~N} & 1.302951 & -0.957721 & 0.000044 \\ \mathrm{C} & 0.670793 & 0.227355 & 0.000097 \\ \mathrm{C} & -0.709298 & 0.245487 & 0.000111 \\ \mathrm{H} & 0.344672 & -1.844300 & 0.000018 \\ \mathrm{O} & 1.376924 & 2.555285 & 0.000183 \\ \mathrm{C} & -1.421621 & -0.998705 & 0.000068 \\ \mathrm{~N} & -1.622481 & 1.288760 & 0.000161 \\ \mathrm{H} & -1.367492 & 2.262813 & 0.000197 \\ \mathrm{C} & -2.894807 & 0.759359 & 0.000151 \\ \mathrm{C} & -2.818044 & -0.664232 & 0.000094 \\ \mathrm{O} & -0.818795 & -2.136615 & 0.000017 \\ \mathrm{C} & -4.131840 & 1.408987 & 0.000188 \\ \mathrm{C} & -3.989187 & -1.435045 & 0.000073 \\ \mathrm{C} & -5.274401 & 0.620881 & 0.000167 \\ \mathrm{C} & -5.211885 & -0.787398 & 0.000110 \\ \mathrm{H} & -4.198366 & 2.490689 & 0.000232 \\ \mathrm{H} & -6.244755 & 1.104706 & 0.000195 \\ \mathrm{H} & -6.131043 & -1.360513 & 0.000095 \\ \mathrm{H} & -3.923034 & -2.516570 & 0.000029\end{array}$


$\mathrm{S}_{1}$ Ind $\left(\mathrm{TS}_{12}\right)$, gas phase

$\begin{array}{lrrr}\mathrm{C} & 2.922918 & 0.698567 & -0.000043 \\ \mathrm{C} & 4.228011 & 1.143105 & -0.000050 \\ \mathrm{C} & 5.259190 & 0.197447 & -0.000069 \\ \mathrm{C} & 4.988759 & -1.201161 & -0.000082 \\ \mathrm{C} & 3.704602 & -1.682237 & -0.000075 \\ \mathrm{C} & 2.636913 & -0.731686 & -0.000056 \\ \mathrm{H} & 4.440826 & 2.205857 & -0.000041 \\ \mathrm{H} & 6.291051 & 0.528554 & -0.000075 \\ \mathrm{H} & 5.822527 & -1.893310 & -0.000096 \\ \mathrm{H} & 3.487400 & -2.743238 & -0.000085 \\ \mathrm{C} & 1.621498 & 1.411100 & -0.000025 \\ \mathrm{~N} & 1.333228 & -0.953504 & -0.000047 \\ \mathrm{C} & 0.682736 & 0.331571 & -0.000028 \\ \mathrm{C} & -0.688853 & 0.269911 & -0.000017 \\ \mathrm{H} & 0.393180 & -1.820336 & -0.000048 \\ \mathrm{O} & 1.387909 & 2.635019 & -0.000010 \\ \mathrm{C} & -1.393366 & -0.996921 & -0.000025 \\ \mathrm{~N} & -1.613935 & 1.292991 & 0.000001 \\ \mathrm{H} & -1.353884 & 2.267485 & 0.000008 \\ \mathrm{C} & -2.885252 & 0.753028 & 0.000005 \\ \mathrm{C} & -2.791644 & -0.668921 & -0.000011 \\ \mathrm{O} & -0.805249 & -2.138295 & -0.000042 \\ \mathrm{C} & -4.125269 & 1.388943 & 0.000021 \\ \mathrm{C} & -3.953412 & -1.453298 & -0.000010 \\ \mathrm{C} & -5.262444 & 0.587025 & 0.000022 \\ \mathrm{C} & -5.184697 & -0.818567 & 0.000007 \\ \mathrm{H} & -4.203023 & 2.469806 & 0.000033 \\ \mathrm{H} & -6.237552 & 1.061175 & 0.000035 \\ \mathrm{H} & -6.097253 & -1.402219 & 0.000008 \\ \mathrm{H} & -3.874482 & -2.533979 & -0.000021\end{array}$


$\mathrm{S}_{0}$ NHxInd (isomer 1), gas phase

$\begin{array}{lrrr}\mathrm{C} & 2.952828 & -1.698193 & -0.265631 \\ \mathrm{C} & 3.549568 & -2.949892 & -0.393071 \\ \mathrm{C} & 4.888642 & -3.084941 & -0.042949 \\ \mathrm{C} & 5.607803 & -1.974306 & 0.425598 \\ \mathrm{C} & 5.024490 & -0.715617 & 0.556129 \\ \mathrm{C} & 3.681471 & -0.591883 & 0.202595 \\ \mathrm{H} & 2.968647 & -3.789528 & -0.757170 \\ \mathrm{H} & 5.381791 & -4.045432 & -0.129050 \\ \mathrm{H} & 6.651185 & -2.096946 & 0.694851 \\ \mathrm{H} & 5.594451 & 0.131704 & 0.918282 \\ \mathrm{C} & 1.583438 & -1.256320 & -0.553285 \\ \mathrm{~N} & 2.866257 & 0.521893 & 0.227468 \\ \mathrm{C} & 1.580525 & 0.222947 & -0.199709 \\ \mathrm{C} & 0.603399 & 1.190472 & -0.188138 \\ \mathrm{H} & 3.088736 & 1.452271 & 0.568327 \\ \mathrm{O} & 0.655898 & -1.931788 & -0.970261 \\ \mathrm{C} & 0.892140 & 2.567461 & 0.350969 \\ \mathrm{~N} & -0.733431 & 1.170044 & -0.578888 \\ \mathrm{C} & -1.314124 & 2.406349 & -0.300206 \\ \mathrm{C} & -0.371507 & 3.285235 & 0.262299 \\ \mathrm{O} & 1.974751 & 2.968771 & 0.775752 \\ \mathrm{C} & -2.627556 & 2.829406 & -0.519392 \\ \mathrm{C} & -0.711258 & 4.590375 & 0.610840 \\ \mathrm{C} & -2.954205 & 4.136682 & -0.164895 \\ \mathrm{C} & -2.015843 & 5.016572 & 0.395240 \\ \mathrm{H} & -3.377378 & 2.174334 & -0.942932 \\ \mathrm{H} & -3.969880 & 4.480868 & -0.326426 \\ \mathrm{H} & -2.313831 & 6.024131 & 0.658085 \\ \mathrm{H} & 0.039157 & 5.244763 & 1.039062 \\ \mathrm{C} & -1.485550 & 0.094541 & -1.232377 \\ \mathrm{C} & -2.294575 & -0.759375 & -0.255434 \\ \mathrm{H} & -0.782348 & -0.534999 & -1.767163 \\ \mathrm{H} & -2.140951 & 0.567740 & -1.968975 \\ \mathrm{H} & -1.627060 & -1.294664 & 0.421041 \\ \mathrm{H} & -2.987553 & -0.155220 & 0.335488 \\ \mathrm{H} & -2.873442 & -1.502670 & -0.810352\end{array}$


$\mathrm{S}_{1}$ NHxInd (isomer 1), gas phase

$\begin{array}{rrrr}\mathrm{C} & 2.938290 & -1.692066 & -0.353294 \\ \mathrm{C} & 3.585376 & -2.892179 & -0.528933 \\ \mathrm{C} & 4.922485 & -2.999902 & -0.114447 \\ \mathrm{C} & 5.609345 & -1.905782 & 0.476958 \\ \mathrm{C} & 4.991534 & -0.693957 & 0.667320 \\ \mathrm{C} & 3.632625 & -0.582927 & 0.246727 \\ \mathrm{H} & 3.064401 & -3.727854 & -0.980803 \\ \mathrm{H} & 5.450108 & -3.936793 & -0.247463 \\ \mathrm{H} & 6.640571 & -2.036922 & 0.781433 \\ \mathrm{H} & 5.502405 & 0.147636 & 1.118329 \\ \mathrm{C} & 1.548050 & -1.261202 & -0.683154 \\ \mathrm{~N} & 2.808035 & 0.443795 & 0.306151 \\ \mathrm{C} & 1.496052 & 0.104098 & -0.244758 \\ \mathrm{C} & 0.567540 & 1.141162 & -0.186176 \\ \mathrm{H} & 2.943139 & 1.401442 & 0.669538 \\ \mathrm{O} & 0.696112 & -1.994199 & -1.217115 \\ \mathrm{C} & 0.882707 & 2.490807 & 0.382653 \\ \mathrm{~N} & -0.742810 & 1.156951 & -0.609201 \\ \mathrm{C} & -1.309520 & 2.410671 & -0.343053 \\ \mathrm{C} & -0.349196 & 3.250529 & 0.252156 \\ \mathrm{O} & 1.969206 & 2.872579 & 0.859809 \\ \mathrm{C} & -2.599577 & 2.863770 & -0.594452 \\ \mathrm{C} & -0.665532 & 4.562731 & 0.599919 \\ \mathrm{C} & -2.904141 & 4.182138 & -0.241010 \\ \mathrm{C} & -1.954076 & 5.025740 & 0.348618 \\ \mathrm{H} & -3.352396 & 2.229075 & -1.044100 \\ \mathrm{H} & -3.904388 & 4.556181 & -0.428354 \\ \mathrm{H} & -2.227711 & 6.041078 & 0.609785 \\ \mathrm{H} & 0.087105 & 5.195223 & 1.055941 \\ \mathrm{C} & -1.512218 & 0.045447 & -1.175464 \\ \mathrm{C} & -2.306368 & -0.728934 & -0.120772 \\ \mathrm{H} & -0.816618 & -0.634009 & -1.664243 \\ \mathrm{H} & -2.177455 & 0.468362 & -1.932916 \\ \mathrm{H} & -1.627636 & -1.204136 & 0.589772 \\ \mathrm{H} & -2.997351 & -0.084458 & 0.428328 \\ \mathrm{H} & -2.883408 & -1.519309 & -0.608003\end{array}$


$\mathrm{S}_{0}$ NHxInd (isomer 2), gas phase

$\begin{array}{lrrc}\mathrm{C} & 2.961723 & -1.712042 & -0.285992 \\ \mathrm{C} & 3.590166 & -2.940721 & -0.423818 \\ \mathrm{C} & 4.939641 & -3.030612 & -0.073795 \\ \mathrm{C} & 5.617405 & -1.902119 & 0.398869 \\ \mathrm{C} & 4.983637 & -0.661936 & 0.537582 \\ \mathrm{C} & 3.641934 & -0.579507 & 0.188282 \\ \mathrm{H} & 3.041772 & -3.800468 & -0.792604 \\ \mathrm{H} & 5.464494 & -3.973633 & -0.166862 \\ \mathrm{H} & 6.664909 & -1.989437 & 0.666047 \\ \mathrm{H} & 5.518037 & 0.206137 & 0.904190 \\ \mathrm{C} & 1.581343 & -1.287529 & -0.563046 \\ \mathrm{~N} & 2.812971 & 0.552184 & 0.244710 \\ \mathrm{C} & 1.600526 & 0.219545 & -0.179701 \\ \mathrm{C} & 0.583125 & 1.206793 & -0.179268 \\ \mathrm{O} & 0.654505 & -1.948585 & -0.977293 \\ \mathrm{C} & 0.853595 & 2.508561 & 0.323135 \\ \mathrm{~N} & -0.764682 & 1.180307 & -0.588459 \\ \mathrm{C} & -1.315208 & 2.413985 & -0.330133 \\ \mathrm{C} & -0.335513 & 3.273624 & 0.236873 \\ \mathrm{O} & 2.014457 & 2.928019 & 0.794145 \\ \mathrm{C} & -2.619741 & 2.879266 & -0.554712 \\ \mathrm{C} & -0.652164 & 4.599424 & 0.577832 \\ \mathrm{C} & -2.907641 & 4.190230 & -0.210416 \\ \mathrm{C} & -1.937834 & 5.049288 & 0.351864 \\ \mathrm{H} & -3.385982 & 2.241464 & -0.976348 \\ \mathrm{H} & -3.911066 & 4.566651 & -0.375420 \\ \mathrm{H} & -2.211775 & 6.066111 & 0.606080 \\ \mathrm{H} & 0.105817 & 5.243300 & 1.007485 \\ \mathrm{C} & -1.524739 & 0.081511 & -1.187765 \\ \mathrm{H} & -0.838084 & -0.546117 & -1.746828 \\ \mathrm{H} & -2.218374 & 0.529426 & -1.903843 \\ \mathrm{H} & 2.641920 & 2.145831 & 0.707530 \\ \mathrm{C} & -2.281599 & -0.764021 & -0.162672 \\ \mathrm{H} & -2.971895 & -0.155795 & 0.427017 \\ \mathrm{H} & -1.585642 & -1.260244 & 0.515288 \\ \mathrm{H} & -2.857237 & -1.539137 & -0.675902\end{array}$


$\mathrm{S}_{1}$ NHxInd (isomer 2), gas phase

$\begin{array}{rrrr}\mathrm{C} & 2.931444 & -1.720197 & -0.334773 \\ \mathrm{C} & 3.583932 & -2.927467 & -0.522893 \\ \mathrm{C} & 4.937830 & -3.011958 & -0.161212 \\ \mathrm{C} & 5.613205 & -1.913788 & 0.376970 \\ \mathrm{C} & 4.954485 & -0.690245 & 0.571014 \\ \mathrm{C} & 3.619097 & -0.614649 & 0.209288 \\ \mathrm{H} & 3.059533 & -3.779898 & -0.939512 \\ \mathrm{H} & 5.470255 & -3.945944 & -0.302293 \\ \mathrm{H} & 6.658205 & -2.007509 & 0.647938 \\ \mathrm{H} & 5.467017 & 0.168266 & 0.989652 \\ \mathrm{C} & 1.549123 & -1.282028 & -0.584830 \\ \mathrm{~N} & 2.769490 & 0.493810 & 0.308742 \\ \mathrm{C} & 1.568970 & 0.147977 & -0.142017 \\ \mathrm{C} & 0.577219 & 1.212860 & -0.100901 \\ \mathrm{O} & 0.605906 & -1.945983 & -1.042284 \\ \mathrm{C} & 0.838520 & 2.488262 & 0.401585 \\ \mathrm{~N} & -0.746569 & 1.198313 & -0.570245 \\ \mathrm{C} & -1.314284 & 2.439117 & -0.356578 \\ \mathrm{C} & -0.349694 & 3.282106 & 0.250234 \\ \mathrm{O} & 1.973254 & 2.943539 & 0.926031 \\ \mathrm{C} & -2.603051 & 2.896950 & -0.643541 \\ \mathrm{C} & -0.671125 & 4.596480 & 0.568416 \\ \mathrm{C} & -2.909478 & 4.218182 & -0.318988 \\ \mathrm{C} & -1.959543 & 5.057015 & 0.277199 \\ \mathrm{H} & -3.347172 & 2.256911 & -1.099917 \\ \mathrm{H} & -3.900792 & 4.599301 & -0.532183 \\ \mathrm{H} & -2.228138 & 6.078955 & 0.517037 \\ \mathrm{H} & 0.062888 & 5.243875 & 1.032370 \\ \mathrm{C} & -1.490572 & 0.073213 & -1.136586 \\ \mathrm{H} & -0.789316 & -0.571957 & -1.662608 \\ \mathrm{H} & -2.202361 & 0.492210 & -1.850153 \\ \mathrm{H} & 2.612216 & 2.182730 & 0.860766 \\ \mathrm{C} & -2.212917 & -0.754076 & -0.066934 \\ \mathrm{H} & -2.912832 & -0.143441 & 0.508534 \\ \mathrm{H} & -1.493147 & -1.213583 & 0.610181 \\ \mathrm{H} & -2.768794 & -1.557596 & -0.555462\end{array}$


$\mathrm{S}_{0}$ NHxInd $\left(\mathrm{TS}_{12}\right)$, gas phase

$\begin{array}{lrrr}\mathrm{C} & -3.032611 & -1.625457 & 0.309777 \\ \mathrm{C} & -3.729837 & -2.815841 & 0.472252 \\ \mathrm{C} & -5.066026 & -2.857677 & 0.074868 \\ \mathrm{C} & -5.670911 & -1.718111 & -0.469851 \\ \mathrm{C} & -4.976120 & -0.516997 & -0.634840 \\ \mathrm{C} & -3.643491 & -0.481822 & -0.237719 \\ \mathrm{H} & -3.237278 & -3.683044 & 0.897770 \\ \mathrm{H} & -5.639672 & -3.769720 & 0.186140 \\ \mathrm{H} & -6.711028 & -1.767494 & -0.773250 \\ \mathrm{H} & -5.457074 & 0.35712 & -1.056841 \\ \mathrm{C} & -1.643959 & -1.265877 & 0.630030 \\ \mathrm{~N} & -2.751319 & 0.588764 & -0.300858 \\ \mathrm{C} & -1.555540 & 0.204868 & 0.186967 \\ \mathrm{C} & -0.529022 & 1.152871 & 0.182189 \\ \mathrm{H} & -2.615207 & 1.847146 & -0.671984 \\ \mathrm{O} & -0.771854 & -1.950672 & 1.126474 \\ \mathrm{C} & -0.823769 & 2.466233 & -0.340378 \\ \mathrm{~N} & 0.807339 & 1.139908 & 0.599291 \\ \mathrm{C} & 1.351390 & 2.381247 & 0.334089 \\ \mathrm{C} & 0.372963 & 3.237565 & -0.237881 \\ \mathrm{O} & -1.978302 & 2.812810 & -0.797079 \\ \mathrm{C} & 2.653173 & 2.848473 & 0.557218 \\ \mathrm{C} & 0.684329 & 4.561548 & -0.577450 \\ \mathrm{C} & 2.938088 & 4.162305 & 0.212551 \\ \mathrm{C} & 1.969352 & 5.018749 & -0.349294 \\ \mathrm{H} & 3.420496 & 2.213386 & 0.980983 \\ \mathrm{H} & 3.940729 & 4.540211 & 0.379434 \\ \mathrm{H} & 2.239902 & 6.036538 & -0.602648 \\ \mathrm{H} & -0.076506 & 5.200502 & -1.009789 \\ \mathrm{C} & 1.579465 & 0.006817 & 1.111545 \\ \mathrm{C} & 2.284800 & -0.791855 & 0.013512 \\ \mathrm{H} & 0.901951 & -0.642625 & 1.658991 \\ \mathrm{H} & 2.304273 & 0.407683 & 1.824449 \\ \mathrm{H} & 1.556262 & -1.237359 & -0.666549 \\ \mathrm{H} & 2.964833 & -0.164254 & -0.567775 \\ \mathrm{H} & 2.863403 & -1.604274 & 0.461281\end{array}$


$\mathrm{S}_{1}$ NHxInd $\left(\mathrm{TS}_{12}\right)$, gas phase

$\begin{array}{rrrr}\mathrm{C} & -2.993983 & -1.609382 & 0.327277 \\ \mathrm{C} & -3.705903 & -2.780691 & 0.483393 \\ \mathrm{C} & -5.045320 & -2.804715 & 0.082036 \\ \mathrm{C} & -5.676029 & -1.656644 & -0.477241 \\ \mathrm{C} & -4.996589 & -0.476726 & -0.646227 \\ \mathrm{C} & -3.626748 & -0.436747 & -0.241122 \\ \mathrm{H} & -3.228275 & -3.655127 & 0.909765 \\ \mathrm{H} & -5.624345 & -3.713547 & 0.196815 \\ \mathrm{H} & -6.716273 & -1.725468 & -0.773121 \\ \mathrm{H} & -5.465975 & 0.402076 & -1.071005 \\ \mathrm{C} & -1.593918 & -1.256385 & 0.645251 \\ \mathrm{~N} & -2.775382 & 0.570742 & -0.301971 \\ \mathrm{C} & -1.504614 & 0.124593 & 0.225957 \\ \mathrm{C} & -0.532149 & 1.116066 & 0.185326 \\ \mathrm{H} & -2.636338 & 1.805015 & -0.661647 \\ \mathrm{O} & -0.737226 & -2.007131 & 1.152723 \\ \mathrm{C} & -0.845032 & 2.442859 & -0.343184 \\ \mathrm{~N} & 0.799079 & 1.117818 & 0.585614 \\ \mathrm{C} & 1.338801 & 2.365681 & 0.322284 \\ \mathrm{C} & 0.352639 & 3.215331 & -0.243102 \\ \mathrm{O} & -1.989919 & 2.806651 & -0.797415 \\ \mathrm{C} & 2.634737 & 2.837131 & 0.544199 \\ \mathrm{C} & 0.653167 & 4.541182 & -0.582361 \\ \mathrm{C} & 2.911792 & 4.156720 & 0.200264 \\ \mathrm{C} & 1.937891 & 5.006613 & -0.357219 \\ \mathrm{H} & 3.405045 & 2.204877 & 0.966613 \\ \mathrm{H} & 3.912478 & 4.540143 & 0.366235 \\ \mathrm{H} & 2.200921 & 6.026518 & -0.610014 \\ \mathrm{H} & -0.113496 & 5.175535 & -1.011153 \\ \mathrm{C} & 1.568413 & -0.016811 & 1.106191 \\ \mathrm{C} & 2.331358 & -0.773839 & 0.017436 \\ \mathrm{H} & 0.870172 & -0.697166 & 1.591080 \\ \mathrm{H} & 2.252379 & 0.376337 & 1.863222 \\ \mathrm{H} & 1.633791 & -1.219672 & -0.694036 \\ \mathrm{H} & 3.022179 & -0.124703 & -0.526768 \\ \mathrm{H} & 2.905895 & -1.585478 & 0.471529\end{array}$


$\mathrm{S}_{0}$ Ind (isomer 1), THF

$\begin{array}{cccc}\mathrm{C} & -2.920709 & 0.674394 & -0.000125 \\ \mathrm{C} & -4.166082 & 1.303147 & -0.000229 \\ \mathrm{C} & -5.310891 & 0.513920 & -0.000298 \\ \mathrm{C} & -5.201956 & -0.886589 & -0.000258 \\ \mathrm{C} & -3.967377 & -1.532988 & -0.000146 \\ \mathrm{C} & -2.824541 & -0.734877 & -0.000079 \\ \mathrm{H} & -4.230202 & 2.385622 & -0.000261 \\ \mathrm{H} & -6.291239 & 0.975093 & -0.000382 \\ \mathrm{H} & -6.105543 & -1.486515 & -0.000311 \\ \mathrm{H} & -3.898648 & -2.614369 & -0.000109 \\ \mathrm{C} & -1.556109 & 1.211302 & -0.000045 \\ \mathrm{~N} & -1.492198 & -1.114655 & 0.000072 \\ \mathrm{C} & -0.680583 & -0.000418 & 0.000080 \\ \mathrm{C} & 0.680552 & 0.000443 & 0.000109 \\ \mathrm{H} & -1.135276 & -2.060095 & -0.000016 \\ \mathrm{O} & -1.157566 & 2.373236 & -0.000037 \\ \mathrm{C} & 1.556084 & -1.211274 & -0.000012 \\ \mathrm{~N} & 1.492161 & 1.114683 & 0.000172 \\ \mathrm{H} & 1.135230 & 2.060121 & -0.000305 \\ \mathrm{C} & 2.824507 & 0.734913 & -0.000093 \\ \mathrm{C} & 2.920681 & -0.674358 & -0.000136 \\ \mathrm{O} & 1.157548 & -2.373208 & 0.000014 \\ \mathrm{C} & 3.967338 & 1.533030 & -0.000216 \\ \mathrm{C} & 4.166057 & -1.303105 & -0.000275 \\ \mathrm{C} & 5.201921 & 0.886636 & -0.000378 \\ \mathrm{C} & 5.310862 & -0.513872 & -0.000402 \\ \mathrm{H} & 3.898605 & 2.614410 & -0.000189 \\ \mathrm{H} & 6.105504 & 1.486567 & -0.000483 \\ \mathrm{H} & 6.291213 & -0.975041 & -0.000524 \\ \mathrm{H} & 4.230182 & -2.385580 & -0.000301\end{array}$


$\mathrm{S}_{1}$ Ind (isomer 1), THF

$\begin{array}{rrrr}\mathrm{C} & -2.916972 & 0.689172 & -0.000216 \\ \mathrm{C} & -4.165283 & 1.299834 & -0.000089 \\ \mathrm{C} & -5.310150 & 0.493266 & -0.000007 \\ \mathrm{C} & -5.207510 & -0.905204 & -0.000047 \\ \mathrm{C} & -3.963398 & -1.540262 & -0.000161 \\ \mathrm{C} & -2.830849 & -0.728950 & -0.000231 \\ \mathrm{H} & -4.248247 & 2.380961 & -0.000079 \\ \mathrm{H} & -6.290676 & 0.955082 & 0.000079 \\ \mathrm{H} & -6.108925 & -1.506807 & 0.000017 \\ \mathrm{H} & -3.880179 & -2.620756 & -0.000179 \\ \mathrm{C} & -1.545985 & 1.226121 & -0.000379 \\ \mathrm{~N} & -1.499995 & -1.086508 & -0.000293 \\ \mathrm{C} & -0.696134 & 0.029994 & -0.000283 \\ \mathrm{C} & 0.688659 & -0.043127 & -0.000224 \\ \mathrm{H} & -1.116493 & -2.027816 & -0.000292 \\ \mathrm{O} & -1.150910 & 2.405066 & -0.000271 \\ \mathrm{C} & 1.538030 & -1.239803 & -0.000159 \\ \mathrm{~N} & 1.493016 & 1.072879 & -0.000213 \\ \mathrm{H} & 1.110206 & 2.014437 & -0.000243 \\ \mathrm{C} & 2.823847 & 0.714598 & -0.000184 \\ \mathrm{C} & 2.909221 & -0.703582 & -0.000172 \\ \mathrm{O} & 1.142245 & -2.418478 & -0.000180 \\ \mathrm{C} & 3.956732 & 1.525293 & -0.000184 \\ \mathrm{C} & 4.157229 & -1.314860 & -0.000167 \\ \mathrm{C} & 5.200620 & 0.889582 & -0.000171 \\ \mathrm{C} & 5.302540 & -0.508884 & -0.000163 \\ \mathrm{H} & 3.874109 & 2.605835 & -0.000198 \\ \mathrm{H} & 6.102274 & 1.490829 & -0.000170 \\ \mathrm{H} & 6.282778 & -0.971316 & -0.000152 \\ \mathrm{H} & 4.239732 & -2.396026 & -0.000156\end{array}$


$\mathrm{S}_{0}$ Ind (isomer 2), $\mathrm{THF}$

$\begin{array}{lrrr}\mathrm{C} & -2.954776 & 0.710010 & -0.000186 \\ \mathrm{C} & -4.224686 & 1.269153 & -0.000079 \\ \mathrm{C} & -5.325039 & 0.405821 & -0.000029 \\ \mathrm{C} & -5.131781 & -0.978266 & -0.000079 \\ \mathrm{C} & -3.850037 & -1.546309 & -0.000174 \\ \mathrm{C} & -2.761732 & -0.687035 & -0.000224 \\ \mathrm{H} & -4.358639 & 2.345394 & -0.000049 \\ \mathrm{H} & -6.330743 & 0.808816 & 0.000046 \\ \mathrm{H} & -5.996414 & -1.633035 & -0.000034 \\ \mathrm{H} & -3.711707 & -2.621122 & -0.000197 \\ \mathrm{C} & -1.608685 & 1.305479 & -0.000296 \\ \mathrm{~N} & -1.392441 & -1.046274 & -0.000280 \\ \mathrm{C} & -0.699071 & 0.071732 & -0.000267 \\ \mathrm{C} & 0.706708 & 0.085296 & -0.000248 \\ \mathrm{O} & -1.240326 & 2.463105 & -0.000197 \\ \mathrm{C} & 1.503700 & -1.071438 & -0.000297 \\ \mathrm{~N} & 1.545184 & 1.195502 & -0.000157 \\ \mathrm{H} & 1.223358 & 2.151998 & -0.000280 \\ \mathrm{C} & 2.844822 & 0.769430 & -0.000165 \\ \mathrm{C} & 2.863827 & -0.660089 & -0.000222 \\ \mathrm{O} & 1.033765 & -2.319779 & -0.000392 \\ \mathrm{C} & 4.035390 & 1.507670 & -0.000106 \\ \mathrm{C} & 4.092821 & -1.347745 & -0.000211 \\ \mathrm{C} & 5.227826 & 0.803012 & -0.000101 \\ \mathrm{C} & 5.261536 & -0.611488 & -0.000152 \\ \mathrm{H} & 4.019315 & 2.591376 & -0.000065 \\ \mathrm{H} & 6.163433 & 1.351229 & -0.000058 \\ \mathrm{H} & 6.219774 & -1.117701 & -0.000146 \\ \mathrm{H} & 4.114070 & -2.431394 & -0.000254 \\ \mathrm{H} & 0.047188 & -2.251001 & -0.000467\end{array}$


$\mathrm{S}_{1}$ Ind (isomer 2), THF

$\begin{array}{lrrr}\mathrm{C} & -2.928629 & 0.739678 & -0.000213 \\ \mathrm{C} & -4.206776 & 1.265755 & -0.000003 \\ \mathrm{C} & -5.294967 & 0.377328 & 0.000090 \\ \mathrm{C} & -5.106098 & -1.018343 & -0.000018 \\ \mathrm{C} & -3.830107 & -1.566216 & -0.000208 \\ \mathrm{C} & -2.732783 & -0.684754 & -0.000300 \\ \mathrm{H} & -4.370838 & 2.337838 & 0.000057 \\ \mathrm{H} & -6.304438 & 0.772815 & 0.000239 \\ \mathrm{H} & -5.971635 & -1.670371 & 0.000062 \\ \mathrm{H} & -3.670051 & -2.638347 & -0.000270 \\ \mathrm{C} & -1.581409 & 1.338586 & -0.000439 \\ \mathrm{~N} & -1.412567 & -1.014292 & -0.000407 \\ \mathrm{C} & -0.714543 & 0.160802 & -0.000371 \\ \mathrm{C} & 0.690046 & 0.111735 & -0.000325 \\ \mathrm{O} & -1.225625 & 2.533602 & -0.000308 \\ \mathrm{C} & 1.484123 & -1.064280 & -0.000321 \\ \mathrm{~N} & 1.532807 & 1.199785 & -0.000292 \\ \mathrm{H} & 1.200984 & 2.155904 & -0.000299 \\ \mathrm{C} & 2.840378 & 0.768338 & -0.000210 \\ \mathrm{C} & 2.844953 & -0.660495 & -0.000217 \\ \mathrm{O} & 1.020871 & -2.311283 & -0.000395 \\ \mathrm{C} & 4.026335 & 1.497825 & -0.000130 \\ \mathrm{C} & 4.067885 & -1.357110 & -0.000149 \\ \mathrm{C} & 5.221869 & 0.782764 & -0.000060 \\ \mathrm{C} & 5.244999 & -0.626498 & -0.000072 \\ \mathrm{H} & 4.017601 & 2.581443 & -0.000123 \\ \mathrm{H} & 6.159689 & 1.326684 & -0.000000 \\ \mathrm{H} & 6.198567 & -1.141230 & -0.000022 \\ \mathrm{H} & 4.082700 & -2.440747 & -0.000163 \\ \mathrm{H} & 0.033298 & -2.244570 & -0.000499\end{array}$




$\begin{array}{lrrr}\mathrm{S}_{0} & \text { Ind }\left(\mathrm{TS}_{12}\right), \mathrm{THF} & \\ & & & \\ & & & \\ \mathrm{C} & 2.967536 & 0.680950 & 0.000094 \\ \mathrm{C} & 4.276831 & 1.148432 & 0.000098 \\ \mathrm{C} & 5.314276 & 0.215140 & 0.000050 \\ \mathrm{C} & 5.026035 & -1.155103 & -0.000000 \\ \mathrm{C} & 3.712781 & -1.636466 & -0.000004 \\ \mathrm{C} & 2.682310 & -0.703657 & 0.000043 \\ \mathrm{H} & 4.481564 & 2.213592 & 0.000137 \\ \mathrm{H} & 6.345256 & 0.548011 & 0.000051 \\ \mathrm{H} & 5.844565 & -1.866801 & -0.000037 \\ \mathrm{H} & 3.505148 & -2.700010 & -0.000043 \\ \mathrm{C} & 1.674893 & 1.385153 & 0.000135 \\ \mathrm{~N} & 1.301430 & -0.939537 & 0.000049 \\ \mathrm{C} & 0.671753 & 0.246577 & 0.000102 \\ \mathrm{C} & -0.711912 & 0.260772 & 0.000114 \\ \mathrm{H} & 0.340081 & -1.831068 & 0.000022 \\ \mathrm{O} & 1.407365 & 2.572545 & 0.000185 \\ \mathrm{C} & -1.419490 & -0.987978 & 0.000070 \\ \mathrm{~N} & -1.633552 & 1.294913 & 0.000163 \\ \mathrm{H} & -1.406468 & 2.277707 & 0.000199 \\ \mathrm{C} & -2.899786 & 0.758140 & 0.000151 \\ \mathrm{C} & -2.815720 & -0.665882 & 0.000093 \\ \mathrm{O} & -0.811203 & -2.128174 & 0.000020 \\ \mathrm{C} & -4.139954 & 1.404595 & 0.000188 \\ \mathrm{C} & -3.985809 & -1.443192 & 0.000071 \\ \mathrm{C} & -5.278507 & 0.611372 & 0.000165 \\ \mathrm{C} & -5.209909 & -0.798934 & 0.000106 \\ \mathrm{H} & -4.206614 & 2.486227 & 0.000233 \\ \mathrm{H} & -6.251510 & 1.090233 & 0.000193 \\ \mathrm{H} & -6.127326 & -1.375484 & 0.000090 \\ \mathrm{H} & -3.921834 & -2.525315 & 0.000026\end{array}$

S40 (S169) 


$\begin{array}{lrrr}\mathrm{C} & 2.947592 & 0.706143 & -0.000045 \\ \mathrm{C} & 4.260838 & 1.133477 & -0.000050 \\ \mathrm{C} & 5.281890 & 0.168912 & -0.000067 \\ \mathrm{C} & 4.990719 & -1.211333 & -0.000079 \\ \mathrm{C} & 3.683079 & -1.667780 & -0.000074 \\ \mathrm{C} & 2.651716 & -0.703124 & -0.000057 \\ \mathrm{H} & 4.501134 & 2.190953 & -0.000040 \\ \mathrm{H} & 6.317223 & 0.489294 & -0.000071 \\ \mathrm{H} & 5.807260 & -1.923639 & -0.000092 \\ \mathrm{H} & 3.447787 & -2.725511 & -0.000084 \\ \mathrm{C} & 1.654190 & 1.429818 & -0.000028 \\ \mathrm{~N} & 1.320269 & -0.898755 & -0.000049 \\ \mathrm{C} & 0.685552 & 0.348757 & -0.000031 \\ \mathrm{C} & -0.700502 & 0.286560 & -0.000021 \\ \mathrm{H} & 0.401790 & -1.781731 & -0.000051 \\ \mathrm{O} & 1.433033 & 2.653134 & -0.000013 \\ \mathrm{C} & -1.394763 & -0.985690 & -0.000027 \\ \mathrm{~N} & -1.632946 & 1.294651 & -0.000002 \\ \mathrm{H} & -1.409980 & 2.279759 & 0.000007 \\ \mathrm{C} & -2.903482 & 0.746123 & 0.000004 \\ \mathrm{C} & -2.795443 & -0.674822 & -0.000012 \\ \mathrm{O} & -0.791771 & -2.125055 & -0.000044 \\ \mathrm{C} & -4.142456 & 1.378311 & 0.000023 \\ \mathrm{C} & -3.954675 & -1.465520 & -0.000010 \\ \mathrm{C} & -5.279409 & 0.569628 & 0.000025 \\ \mathrm{C} & -5.191069 & -0.834405 & 0.000009 \\ \mathrm{H} & -4.221543 & 2.459145 & 0.000036 \\ \mathrm{H} & -6.257053 & 1.038512 & 0.000040 \\ \mathrm{H} & -6.099657 & -1.424996 & 0.000010 \\ \mathrm{H} & -3.878891 & -2.546907 & -0.000022 \\ & & & \end{array}$


$\mathrm{S}_{0}$ NHxInd (isomer 1), THF

$\begin{array}{lrrr}\mathrm{C} & 3.001193 & -1.654596 & -0.457054 \\ \mathrm{C} & 3.661156 & -2.847809 & -0.749745 \\ \mathrm{C} & 4.953039 & -3.026570 & -0.266792 \\ \mathrm{C} & 5.567627 & -2.016990 & 0.493209 \\ \mathrm{C} & 4.922491 & -0.818415 & 0.791151 \\ \mathrm{C} & 3.626118 & -0.651433 & 0.303880 \\ \mathrm{H} & 3.170104 & -3.612786 & -1.340895 \\ \mathrm{H} & 5.491614 & -3.943156 & -0.475771 \\ \mathrm{H} & 6.576532 & -2.172653 & 0.859767 \\ \mathrm{H} & 5.408863 & -0.047235 & 1.376733 \\ \mathrm{C} & 1.667501 & -1.175738 & -0.834236 \\ \mathrm{~N} & 2.768445 & 0.418436 & 0.448603 \\ \mathrm{C} & 1.555008 & 0.193329 & -0.183587 \\ \mathrm{C} & 0.567474 & 1.153343 & -0.155024 \\ \mathrm{H} & 2.960709 & 1.297795 & 0.916388 \\ \mathrm{O} & 0.854759 & -1.741885 & -1.553019 \\ \mathrm{C} & 0.830332 & 2.511669 & 0.441538 \\ \mathrm{~N} & -0.733669 & 1.158440 & -0.647641 \\ \mathrm{C} & -1.306435 & 2.410516 & -0.416378 \\ \mathrm{C} & -0.390839 & 3.270116 & 0.216880 \\ \mathrm{O} & 1.871646 & 2.878448 & 0.988173 \\ \mathrm{C} & -2.593995 & 2.856397 & -0.725598 \\ \mathrm{C} & -0.728916 & 4.584972 & 0.537763 \\ \mathrm{C} & -2.917462 & 4.172970 & -0.403152 \\ \mathrm{C} & -2.003017 & 5.037876 & 0.219616 \\ \mathrm{H} & -3.324563 & 2.214514 & -1.199839 \\ \mathrm{H} & -3.911080 & 4.537196 & -0.640829 \\ \mathrm{H} & -2.296899 & 6.054359 & 0.452239 \\ \mathrm{H} & -0.006055 & 5.228906 & 1.025981 \\ \mathrm{C} & -1.549450 & 0.004211 & -1.046214 \\ \mathrm{C} & -2.242059 & -0.674699 & 0.136226 \\ \mathrm{H} & -0.901642 & -0.700325 & -1.558078 \\ \mathrm{H} & -2.285228 & 0.365313 & -1.767062 \\ \mathrm{H} & -1.511128 & -1.064455 & 0.849453 \\ \mathrm{H} & -2.908491 & 0.011331 & 0.665615 \\ \mathrm{H} & -2.838999 & -1.516607 & -0.226577\end{array}$

S42 (S169) 
$\mathrm{S}_{1}$ NHxInd (isomer 1), THF

$\begin{array}{rrrr}\mathrm{C} & 2.941418 & -1.704786 & -0.348980 \\ \mathrm{C} & 3.587412 & -2.918969 & -0.542247 \\ \mathrm{C} & 4.923621 & -3.037421 & -0.140893 \\ \mathrm{C} & 5.600141 & -1.956440 & 0.445981 \\ \mathrm{C} & 4.967535 & -0.729400 & 0.648677 \\ \mathrm{C} & 3.636179 & -0.624373 & 0.243430 \\ \mathrm{H} & 3.065909 & -3.754341 & -0.995942 \\ \mathrm{H} & 5.445583 & -3.976253 & -0.285572 \\ \mathrm{H} & 6.634133 & -2.075730 & 0.748208 \\ \mathrm{H} & 5.485179 & 0.106762 & 1.103957 \\ \mathrm{C} & 1.568696 & -1.264908 & -0.651521 \\ \mathrm{~N} & 2.782749 & 0.442966 & 0.318641 \\ \mathrm{C} & 1.526764 & 0.147003 & -0.188048 \\ \mathrm{C} & 0.576544 & 1.181404 & -0.140414 \\ \mathrm{H} & 2.968903 & 1.380015 & 0.693776 \\ \mathrm{O} & 0.674838 & -1.946173 & -1.174280 \\ \mathrm{C} & 0.872692 & 2.520090 & 0.416017 \\ \mathrm{~N} & -0.739577 & 1.183348 & -0.583661 \\ \mathrm{C} & -1.315061 & 2.427934 & -0.343745 \\ \mathrm{C} & -0.363436 & 3.278359 & 0.260595 \\ \mathrm{O} & 1.951844 & 2.912080 & 0.907057 \\ \mathrm{C} & -2.610282 & 2.866227 & -0.617316 \\ \mathrm{C} & -0.698675 & 4.586463 & 0.593598 \\ \mathrm{C} & -2.932281 & 4.183131 & -0.277911 \\ \mathrm{C} & -1.993128 & 5.035478 & 0.318690 \\ \mathrm{H} & -3.349634 & 2.220423 & -1.072849 \\ \mathrm{H} & -3.932344 & 4.548589 & -0.480744 \\ \mathrm{H} & -2.275993 & 6.051317 & 0.569173 \\ \mathrm{H} & 0.033054 & 5.238467 & 1.057125 \\ \mathrm{C} & -1.495481 & 0.071463 & -1.164314 \\ \mathrm{C} & -2.244818 & -0.745016 & -0.107840 \\ \mathrm{H} & -0.794460 & -0.574827 & -1.686873 \\ \mathrm{H} & -2.192545 & 0.500030 & -1.886125 \\ \mathrm{H} & -1.548188 & -1.195618 & 0.601379 \\ \mathrm{H} & -2.961469 & -0.130701 & 0.442615 \\ \mathrm{H} & -2.791137 & -1.551805 & -0.603921 \\ & & & \end{array}$

S43 (S169) 
$\mathrm{S}_{0}$ NHxInd (isomer 2), THF

$\begin{array}{rrrc}\mathrm{C} & 2.972454 & -1.703316 & -0.354874 \\ \mathrm{C} & 3.624625 & -2.914339 & -0.541780 \\ \mathrm{C} & 4.957596 & -3.011672 & -0.132487 \\ \mathrm{C} & 5.597675 & -1.909861 & 0.444667 \\ \mathrm{C} & 4.940574 & -0.687135 & 0.632241 \\ \mathrm{C} & 3.616271 & -0.598663 & 0.224062 \\ \mathrm{H} & 3.112837 & -3.757967 & -0.991932 \\ \mathrm{H} & 5.498585 & -3.941448 & -0.261661 \\ \mathrm{H} & 6.632387 & -2.003146 & 0.756122 \\ \mathrm{H} & 5.446573 & 0.160063 & 1.080224 \\ \mathrm{C} & 1.601918 & -1.274187 & -0.671328 \\ \mathrm{~N} & 2.768119 & 0.517148 & 0.314881 \\ \mathrm{C} & 1.578475 & 0.202975 & -0.180660 \\ \mathrm{C} & 0.561634 & 1.193860 & -0.175534 \\ \mathrm{O} & 0.713907 & -1.912978 & -1.193006 \\ \mathrm{C} & 0.836457 & 2.491356 & 0.336750 \\ \mathrm{~N} & -0.775420 & 1.184392 & -0.614220 \\ \mathrm{C} & -1.316761 & 2.423332 & -0.366812 \\ \mathrm{C} & -0.337181 & 3.274128 & 0.217606 \\ \mathrm{O} & 1.995895 & 2.894371 & 0.832426 \\ \mathrm{C} & -2.615724 & 2.899744 & -0.608092 \\ \mathrm{C} & -0.646321 & 4.606398 & 0.549199 \\ \mathrm{C} & -2.895455 & 4.214347 & -0.271612 \\ \mathrm{C} & -1.924018 & 5.067404 & 0.301427 \\ \mathrm{H} & -3.381247 & 2.267698 & -1.039440 \\ \mathrm{H} & -3.893057 & 4.599975 & -0.451283 \\ \mathrm{H} & -2.191798 & 6.088285 & 0.547385 \\ \mathrm{H} & 0.107240 & 5.248706 & 0.990108 \\ \mathrm{C} & -1.560009 & 0.060157 & -1.134168 \\ \mathrm{H} & -0.900644 & -0.570797 & -1.721166 \\ \mathrm{H} & -2.304319 & 0.479673 & -1.813264 \\ \mathrm{H} & 2.605329 & 2.091850 & 0.771050 \\ \mathrm{C} & -2.237962 & -0.761242 & -0.038701 \\ \mathrm{H} & -2.922378 & -0.149352 & 0.554804 \\ \mathrm{H} & -1.500952 & -1.206017 & 0.633758 \\ \mathrm{H} & -2.812507 & -1.573987 & -0.492636\end{array}$


$\mathrm{S}_{1}$ NHxInd (isomer 2), THF

$\begin{array}{rrrr}\mathrm{C} & 2.944476 & -1.713598 & -0.353905 \\ \mathrm{C} & 3.615150 & -2.912432 & -0.527120 \\ \mathrm{C} & 4.959981 & -2.984651 & -0.129478 \\ \mathrm{C} & 5.614333 & -1.876612 & 0.430690 \\ \mathrm{C} & 4.947133 & -0.664150 & 0.609810 \\ \mathrm{C} & 3.608341 & -0.594238 & 0.212748 \\ \mathrm{H} & 3.117854 & -3.774001 & -0.959347 \\ \mathrm{H} & 5.504679 & -3.913377 & -0.257627 \\ \mathrm{H} & 6.652806 & -1.965444 & 0.727971 \\ \mathrm{H} & 5.442928 & 0.197206 & 1.043246 \\ \mathrm{C} & 1.564693 & -1.288034 & -0.646474 \\ \mathrm{~N} & 2.764000 & 0.486318 & 0.292821 \\ \mathrm{C} & 1.552462 & 0.132002 & -0.195944 \\ \mathrm{C} & 0.566048 & 1.177863 & -0.160836 \\ \mathrm{O} & 0.656261 & -1.972611 & -1.145532 \\ \mathrm{C} & 0.848364 & 2.477550 & 0.351740 \\ \mathrm{~N} & -0.754406 & 1.173571 & -0.587213 \\ \mathrm{C} & -1.310706 & 2.424562 & -0.350204 \\ \mathrm{C} & -0.329229 & 3.265901 & 0.233533 \\ \mathrm{O} & 1.999259 & 2.903182 & 0.849467 \\ \mathrm{C} & -2.599090 & 2.888773 & -0.604410 \\ \mathrm{C} & -0.637181 & 4.592489 & 0.563093 \\ \mathrm{C} & -2.887940 & 4.213433 & -0.269000 \\ \mathrm{C} & -1.923010 & 5.055812 & 0.306403 \\ \mathrm{H} & -3.358052 & 2.254988 & -1.044589 \\ \mathrm{H} & -3.883993 & 4.597112 & -0.457772 \\ \mathrm{H} & -2.186216 & 6.077737 & 0.552788 \\ \mathrm{H} & 0.110891 & 5.238042 & 1.008060 \\ \mathrm{C} & -1.525899 & 0.055990 & -1.139883 \\ \mathrm{H} & -0.835350 & -0.604335 & -1.657319 \\ \mathrm{H} & -2.221856 & 0.478051 & -1.866825 \\ \mathrm{H} & 2.617138 & 2.113635 & 0.782936 \\ \mathrm{C} & -2.277587 & -0.731778 & -0.065489 \\ \mathrm{H} & -2.983415 & -0.100016 & 0.479347 \\ \mathrm{H} & -1.582363 & -1.178042 & 0.648159 \\ \mathrm{H} & -2.837698 & -1.541147 & -0.541796 \\ & & & \end{array}$

S45 (S169) 
$\mathrm{S}_{0} \mathrm{NHxInd}\left(\mathrm{TS}_{12}\right), \mathrm{THF}$

$\begin{array}{rrrr}\mathrm{C} & -3.040645 & -1.625720 & 0.334594 \\ \mathrm{C} & -3.749439 & -2.808132 & 0.514926 \\ \mathrm{C} & -5.080671 & -2.849131 & 0.098771 \\ \mathrm{C} & -5.670666 & -1.718565 & -0.481102 \\ \mathrm{C} & -4.964439 & -0.525609 & -0.664013 \\ \mathrm{C} & -3.637471 & -0.492216 & -0.248401 \\ \mathrm{H} & -3.275505 & -3.672576 & 0.967049 \\ \mathrm{H} & -5.661804 & -3.755006 & 0.223085 \\ \mathrm{H} & -6.706662 & -1.767953 & -0.798448 \\ \mathrm{H} & -5.433797 & 0.341488 & -1.113592 \\ \mathrm{C} & -1.655018 & -1.265251 & 0.666238 \\ \mathrm{~N} & -2.736055 & 0.570084 & -0.325509 \\ \mathrm{C} & -1.548742 & 0.193711 & 0.184512 \\ \mathrm{C} & -0.521416 & 1.144649 & 0.175425 \\ \mathrm{H} & -2.599575 & 1.834084 & -0.700625 \\ \mathrm{O} & -0.799950 & -1.942683 & 1.202562 \\ \mathrm{C} & -0.817080 & 2.455986 & -0.350063 \\ \mathrm{~N} & 0.811225 & 1.137357 & 0.600277 \\ \mathrm{C} & 1.350148 & 2.381021 & 0.343228 \\ \mathrm{C} & 0.370848 & 3.234953 & -0.234669 \\ \mathrm{O} & -1.973511 & 2.798631 & -0.818509 \\ \mathrm{C} & 2.650225 & 2.851957 & 0.575948 \\ \mathrm{C} & 0.680646 & 4.563395 & -0.569078 \\ \mathrm{C} & 2.932013 & 4.167490 & 0.236510 \\ \mathrm{C} & 1.962439 & 5.023153 & -0.330249 \\ \mathrm{H} & 3.415352 & 2.217678 & 1.004784 \\ \mathrm{H} & 3.932128 & 4.549179 & 0.410845 \\ \mathrm{H} & 2.231428 & 6.042970 & -0.578589 \\ \mathrm{H} & -0.075170 & 5.206132 & -1.005784 \\ \mathrm{C} & 1.593981 & -0.001399 & 1.089225 \\ \mathrm{C} & 2.289547 & -0.779532 & -0.027384 \\ \mathrm{H} & 0.922440 & -0.656232 & 1.637046 \\ \mathrm{H} & 2.326115 & 0.391147 & 1.797674 \\ \mathrm{H} & 1.562216 & -1.197396 & -0.727553 \\ \mathrm{H} & 2.982994 & -0.146590 & -0.587197 \\ \mathrm{H} & 2.857092 & -1.609416 & 0.403692\end{array}$

S46 (S169) 
$\mathrm{S}_{1}$ NHxInd $\left(\mathrm{TS}_{12}\right)$, THF

$\begin{array}{rrrr}\mathrm{C} & -3.011123 & -1.632557 & 0.329350 \\ \mathrm{C} & -3.737750 & -2.799396 & 0.491450 \\ \mathrm{C} & -5.082872 & -2.810671 & 0.090491 \\ \mathrm{C} & -5.686813 & -1.668985 & -0.464578 \\ \mathrm{C} & -4.971945 & -0.487033 & -0.635382 \\ \mathrm{C} & -3.627607 & -0.477502 & -0.233865 \\ \mathrm{H} & -3.279576 & -3.684249 & 0.919566 \\ \mathrm{H} & -5.668367 & -3.714931 & 0.210859 \\ \mathrm{H} & -6.727361 & -1.711421 & -0.764557 \\ \mathrm{H} & -5.430424 & 0.396564 & -1.064048 \\ \mathrm{C} & -1.610080 & -1.284360 & 0.638576 \\ \mathrm{~N} & -2.730238 & 0.542420 & -0.294775 \\ \mathrm{C} & -1.511710 & 0.124725 & 0.205179 \\ \mathrm{C} & -0.520337 & 1.127271 & 0.164700 \\ \mathrm{H} & -2.621178 & 1.768632 & -0.665520 \\ \mathrm{O} & -0.743969 & -2.016531 & 1.142771 \\ \mathrm{C} & -0.836295 & 2.442515 & -0.358664 \\ \mathrm{~N} & 0.802658 & 1.127831 & 0.567645 \\ \mathrm{C} & 1.347683 & 2.383190 & 0.317888 \\ \mathrm{C} & 0.357864 & 3.226415 & -0.252035 \\ \mathrm{O} & -1.990837 & 2.792470 & -0.815645 \\ \mathrm{C} & 2.638654 & 2.848978 & 0.554974 \\ \mathrm{C} & 0.661597 & 4.551500 & -0.584650 \\ \mathrm{C} & 2.921952 & 4.174307 & 0.216213 \\ \mathrm{C} & 1.950356 & 5.017666 & -0.346324 \\ \mathrm{H} & 3.402812 & 2.215126 & 0.986630 \\ \mathrm{H} & 3.920472 & 4.558047 & 0.392507 \\ \mathrm{H} & 2.210063 & 6.039692 & -0.595994 \\ \mathrm{H} & -0.094739 & 5.195204 & -1.018959 \\ \mathrm{C} & 1.570966 & -0.002102 & 1.096647 \\ \mathrm{C} & 2.327990 & -0.767041 & 0.009267 \\ \mathrm{H} & 0.872440 & -0.672275 & 1.593331 \\ \mathrm{H} & 2.262925 & 0.396997 & 1.840900 \\ \mathrm{H} & 1.636205 & -1.191040 & -0.721758 \\ \mathrm{H} & 3.041171 & -0.126479 & -0.515256 \\ \mathrm{H} & 2.880636 & -1.591318 & 0.468691\end{array}$

S47 (S169) 
$\mathrm{S}_{0} \mathrm{NHxInd}$ (isomer 1), $\mathrm{MCH}$

$\begin{array}{rrrc}\mathrm{C} & 2.959237 & -1.698161 & -0.290604 \\ \mathrm{C} & 3.566812 & -2.943635 & -0.436909 \\ \mathrm{C} & 4.901007 & -3.077818 & -0.068848 \\ \mathrm{C} & 5.606396 & -1.973483 & 0.435580 \\ \mathrm{C} & 5.013378 & -0.721658 & 0.585095 \\ \mathrm{C} & 3.674849 & -0.598807 & 0.213459 \\ \mathrm{H} & 3.000465 & -3.780932 & -0.828656 \\ \mathrm{H} & 5.401538 & -4.033234 & -0.169104 \\ \mathrm{H} & 6.646392 & -2.096081 & 0.717881 \\ \mathrm{H} & 5.571537 & 0.121498 & 0.974451 \\ \mathrm{C} & 1.593196 & -1.255623 & -0.590964 \\ \mathrm{~N} & 2.853053 & 0.508298 & 0.250875 \\ \mathrm{C} & 1.574957 & 0.214920 & -0.200863 \\ \mathrm{C} & 0.596302 & 1.183291 & -0.184576 \\ \mathrm{H} & 3.074341 & 1.432049 & 0.608902 \\ \mathrm{O} & 0.679899 & -1.926891 & -1.047174 \\ \mathrm{C} & 0.884640 & 2.557786 & 0.362995 \\ \mathrm{~N} & -0.736501 & 1.170049 & -0.585499 \\ \mathrm{C} & -1.313690 & 2.409076 & -0.312722 \\ \mathrm{C} & -0.371929 & 3.283534 & 0.258680 \\ \mathrm{O} & 1.964445 & 2.953811 & 0.802799 \\ \mathrm{C} & -2.623879 & 2.837429 & -0.542733 \\ \mathrm{C} & -0.709534 & 4.591166 & 0.604023 \\ \mathrm{C} & -2.947577 & 4.146259 & -0.191888 \\ \mathrm{C} & -2.009910 & 5.022685 & 0.375962 \\ \mathrm{H} & -3.372589 & 2.185573 & -0.973018 \\ \mathrm{H} & -3.960313 & 4.494888 & -0.362498 \\ \mathrm{H} & -2.305492 & 6.032049 & 0.635097 \\ \mathrm{H} & 0.037135 & 5.245117 & 1.039756 \\ \mathrm{C} & -1.499345 & 0.085237 & -1.211693 \\ \mathrm{C} & -2.293358 & -0.751258 & -0.209306 \\ \mathrm{H} & -0.803859 & -0.551038 & -1.747953 \\ \mathrm{H} & -2.166341 & 0.546858 & -1.944141 \\ \mathrm{H} & -1.622137 & -1.257995 & 0.486397 \\ \mathrm{H} & -2.995855 & -0.141885 & 0.365187 \\ \mathrm{H} & -2.862586 & -1.518285 & -0.742269\end{array}$

S48 (S169) 
$\mathrm{S}_{1} \mathrm{NHxInd}$ (isomer 1), $\mathrm{MCH}$

$\begin{array}{rrrr}\mathrm{C} & 2.941138 & -1.703182 & -0.344867 \\ \mathrm{C} & 3.591263 & -2.908375 & -0.522185 \\ \mathrm{C} & 4.931733 & -3.012263 & -0.120053 \\ \mathrm{C} & 5.612137 & -1.919472 & 0.455903 \\ \mathrm{C} & 4.982299 & -0.700939 & 0.645304 \\ \mathrm{C} & 3.635381 & -0.602718 & 0.238745 \\ \mathrm{H} & 3.073043 & -3.750786 & -0.965307 \\ \mathrm{H} & 5.458633 & -3.949450 & -0.254992 \\ \mathrm{H} & 6.646637 & -2.038124 & 0.754972 \\ \mathrm{H} & 5.494896 & 0.143695 & 1.089643 \\ \mathrm{C} & 1.555986 & -1.271862 & -0.656025 \\ \mathrm{~N} & 2.793434 & 0.440484 & 0.301118 \\ \mathrm{C} & 1.509270 & 0.118591 & -0.216071 \\ \mathrm{C} & 0.569875 & 1.153195 & -0.160913 \\ \mathrm{H} & 2.951410 & 1.390859 & 0.666813 \\ \mathrm{O} & 0.679600 & -1.983714 & -1.172960 \\ \mathrm{C} & 0.879378 & 2.500139 & 0.401167 \\ \mathrm{~N} & -0.742577 & 1.163696 & -0.587758 \\ \mathrm{C} & -1.311581 & 2.417130 & -0.334830 \\ \mathrm{C} & -0.353140 & 3.260760 & 0.261269 \\ \mathrm{O} & 1.964133 & 2.884298 & 0.882034 \\ \mathrm{C} & -2.602121 & 2.865702 & -0.596039 \\ \mathrm{C} & -0.675218 & 4.572882 & 0.600494 \\ \mathrm{C} & -2.912001 & 4.185561 & -0.251209 \\ \mathrm{C} & -1.965415 & 5.031978 & 0.339252 \\ \mathrm{H} & -3.350635 & 2.226993 & -1.047061 \\ \mathrm{H} & -3.911679 & 4.557108 & -0.445869 \\ \mathrm{H} & -2.240827 & 6.048636 & 0.594085 \\ \mathrm{H} & 0.070034 & 5.213537 & 1.057738 \\ \mathrm{C} & -1.504656 & 0.057898 & -1.172959 \\ \mathrm{C} & -2.298689 & -0.735379 & -0.132719 \\ \mathrm{H} & -0.803537 & -0.609823 & -1.668917 \\ \mathrm{H} & -2.171172 & 0.488740 & -1.923770 \\ \mathrm{H} & -1.626799 & -1.204257 & 0.588560 \\ \mathrm{H} & -3.009673 & -0.105623 & 0.407951 \\ \mathrm{H} & -2.855876 & -1.531134 & -0.634819 \\ & & & \end{array}$

S49 (S169) 
$\mathrm{S}_{0}$ NHxInd (isomer 2), $\mathrm{MCH}$

$\begin{array}{rrrr}\mathrm{C} & 2.964797 & -1.713537 & -0.295331 \\ \mathrm{C} & 3.600972 & -2.938229 & -0.439403 \\ \mathrm{C} & 4.948786 & -3.023740 & -0.081475 \\ \mathrm{C} & 5.618553 & -1.896340 & 0.404798 \\ \mathrm{C} & 4.977728 & -0.660251 & 0.549965 \\ \mathrm{C} & 3.637986 & -0.582471 & 0.192834 \\ \mathrm{H} & 3.062449 & -3.799827 & -0.818471 \\ \mathrm{H} & 5.478547 & -3.963600 & -0.179455 \\ \mathrm{H} & 6.664838 & -1.980734 & 0.677667 \\ \mathrm{H} & 5.506074 & 0.207213 & 0.927214 \\ \mathrm{C} & 1.584507 & -1.291861 & -0.577610 \\ \mathrm{~N} & 2.802554 & 0.543901 & 0.253447 \\ \mathrm{C} & 1.593423 & 0.211732 & -0.179572 \\ \mathrm{C} & 0.576521 & 1.200853 & -0.176854 \\ \mathrm{O} & 0.664573 & -1.953000 & -1.007561 \\ \mathrm{C} & 0.851688 & 2.502012 & 0.325821 \\ \mathrm{~N} & -0.770189 & 1.179278 & -0.586416 \\ \mathrm{C} & -1.315667 & 2.415310 & -0.331769 \\ \mathrm{C} & -0.333206 & 3.272609 & 0.236014 \\ \mathrm{O} & 2.015131 & 2.914714 & 0.797948 \\ \mathrm{C} & -2.618824 & 2.884674 & -0.559200 \\ \mathrm{C} & -0.646709 & 4.600888 & 0.574174 \\ \mathrm{C} & -2.902705 & 4.197002 & -0.217604 \\ \mathrm{C} & -1.930525 & 5.054038 & 0.345076 \\ \mathrm{H} & -3.385539 & 2.248464 & -0.982305 \\ \mathrm{H} & -3.904552 & 4.576838 & -0.384883 \\ \mathrm{H} & -2.201499 & 6.072410 & 0.596934 \\ \mathrm{H} & 0.110608 & 5.245599 & 1.004280 \\ \mathrm{C} & -1.534446 & 0.078909 & -1.179567 \\ \mathrm{H} & -0.849605 & -0.548232 & -1.741188 \\ \mathrm{H} & -2.231802 & 0.524379 & -1.892772 \\ \mathrm{H} & 2.636376 & 2.124694 & 0.712723 \\ \mathrm{C} & -2.286199 & -0.762687 & -0.149321 \\ \mathrm{H} & -2.989201 & -0.157580 & 0.428899 \\ \mathrm{H} & -1.592650 & -1.243570 & 0.542868 \\ \mathrm{H} & -2.849994 & -1.550104 & -0.657852 \\ & & & \end{array}$


$\mathrm{S}_{1}$ NHxInd (isomer 2), $\mathrm{MCH}$

$\begin{array}{rrrc}\mathrm{C} & 2.937623 & -1.719581 & -0.335705 \\ \mathrm{C} & 3.599956 & -2.922806 & -0.507677 \\ \mathrm{C} & 4.951856 & -2.994911 & -0.134655 \\ \mathrm{C} & 5.618907 & -1.885628 & 0.399819 \\ \mathrm{C} & 4.955765 & -0.667425 & 0.577947 \\ \mathrm{C} & 3.615479 & -0.600167 & 0.205362 \\ \mathrm{H} & 3.088246 & -3.784944 & -0.920685 \\ \mathrm{H} & 5.491020 & -3.926834 & -0.263161 \\ \mathrm{H} & 6.662440 & -1.973107 & 0.678749 \\ \mathrm{H} & 5.460586 & 0.197779 & 0.992499 \\ \mathrm{C} & 1.553644 & -1.290998 & -0.603666 \\ \mathrm{~N} & 2.769427 & 0.491758 & 0.289082 \\ \mathrm{C} & 1.559029 & 0.137697 & -0.171547 \\ \mathrm{C} & 0.571377 & 1.191437 & -0.134550 \\ \mathrm{O} & 0.625766 & -1.971457 & -1.068994 \\ \mathrm{C} & 0.845964 & 2.481545 & 0.373218 \\ \mathrm{~N} & -0.751155 & 1.181718 & -0.574653 \\ \mathrm{C} & -1.311711 & 2.429364 & -0.346366 \\ \mathrm{C} & -0.336473 & 3.271471 & 0.244468 \\ \mathrm{O} & 1.990575 & 2.919754 & 0.879834 \\ \mathrm{C} & -2.600028 & 2.892106 & -0.612481 \\ \mathrm{C} & -0.649118 & 4.593563 & 0.569341 \\ \mathrm{C} & -2.894924 & 4.215401 & -0.281646 \\ \mathrm{C} & -1.935448 & 5.056233 & 0.300691 \\ \mathrm{H} & -3.354210 & 2.256260 & -1.057870 \\ \mathrm{H} & -3.889267 & 4.598286 & -0.479263 \\ \mathrm{H} & -2.200219 & 6.078284 & 0.544420 \\ \mathrm{H} & 0.094838 & 5.239257 & 1.020293 \\ \mathrm{C} & -1.508446 & 0.065160 & -1.145335 \\ \mathrm{H} & -0.810564 & -0.588464 & -1.662881 \\ \mathrm{H} & -2.201887 & 0.490849 & -1.873096 \\ \mathrm{H} & 2.619134 & 2.144852 & 0.809248 \\ \mathrm{C} & -2.261867 & -0.743484 & -0.086358 \\ \mathrm{H} & -2.969667 & -0.123492 & 0.469428 \\ \mathrm{H} & -1.566326 & -1.203685 & 0.616690 \\ \mathrm{H} & -2.817519 & -1.546039 & -0.578451 \\ & & & \end{array}$




\section{$\mathrm{S}_{0} \mathrm{NHxInd}\left(\mathrm{TS}_{12}\right), \mathrm{MCH}$}

$\begin{array}{lrrr}\mathrm{C} & -3.036688 & -1.628708 & 0.309988 \\ \mathrm{C} & -3.738443 & -2.816898 & 0.474308 \\ \mathrm{C} & -5.075560 & -2.853520 & 0.079040 \\ \mathrm{C} & -5.677822 & -1.712343 & -0.465310 \\ \mathrm{C} & -4.978951 & -0.513570 & -0.632232 \\ \mathrm{C} & -3.645643 & -0.483746 & -0.237286 \\ \mathrm{H} & -3.251537 & -3.687800 & 0.899060 \\ \mathrm{H} & -5.651891 & -3.763801 & 0.191837 \\ \mathrm{H} & -6.718599 & -1.758262 & -0.767035 \\ \mathrm{H} & -5.457757 & 0.362014 & -1.054048 \\ \mathrm{C} & -1.646597 & -1.271955 & 0.626833 \\ \mathrm{~N} & -2.749696 & 0.583224 & -0.302974 \\ \mathrm{C} & -1.554125 & 0.198574 & 0.181715 \\ \mathrm{C} & -0.526894 & 1.148139 & 0.173905 \\ \mathrm{H} & -2.614229 & 1.846355 & -0.673971 \\ \mathrm{O} & -0.776515 & -1.959384 & 1.123947 \\ \mathrm{C} & -0.824184 & 2.462339 & -0.344311 \\ \mathrm{~N} & 0.810743 & 1.135430 & 0.584546 \\ \mathrm{C} & 1.350750 & 2.379050 & 0.326560 \\ \mathrm{C} & 0.369608 & 3.236061 & -0.241665 \\ \mathrm{O} & -1.982134 & 2.809149 & -0.798533 \\ \mathrm{C} & 2.651950 & 2.847968 & 0.553100 \\ \mathrm{C} & 0.679677 & 4.562937 & -0.576218 \\ \mathrm{C} & 2.934320 & 4.163405 & 0.213828 \\ \mathrm{C} & 1.963806 & 5.020468 & -0.345829 \\ \mathrm{H} & 3.418972 & 2.212244 & 0.976418 \\ \mathrm{H} & 3.936010 & 4.543034 & 0.382913 \\ \mathrm{H} & 2.232803 & 6.039835 & -0.595115 \\ \mathrm{H} & -0.079873 & 5.205368 & -1.006143 \\ \mathrm{C} & 1.583728 & 0.006188 & 1.106436 \\ \mathrm{C} & 2.306603 & -0.788725 & 0.018877 \\ \mathrm{H} & 0.902411 & -0.644651 & 1.647026 \\ \mathrm{H} & 2.298368 & 0.410173 & 1.827234 \\ \mathrm{H} & 1.594307 & -1.229336 & -0.681915 \\ \mathrm{H} & 3.002765 & -0.162703 & -0.545143 \\ \mathrm{H} & 2.873541 & -1.604895 & 0.475781\end{array}$




\section{$\mathrm{S}_{1} \mathrm{NHxInd}\left(\mathrm{TS}_{12}\right), \mathrm{MCH}$}

$\begin{array}{rrrr}\mathrm{C} & -3.008269 & -1.619182 & 0.332682 \\ \mathrm{C} & -3.732233 & -2.782678 & 0.496124 \\ \mathrm{C} & -5.074899 & -2.795364 & 0.094142 \\ \mathrm{C} & -5.690124 & -1.649972 & -0.468945 \\ \mathrm{C} & -4.991272 & -0.474747 & -0.644009 \\ \mathrm{C} & -3.629565 & -0.452445 & -0.239795 \\ \mathrm{H} & -3.269630 & -3.663158 & 0.927179 \\ \mathrm{H} & -5.660675 & -3.699057 & 0.215418 \\ \mathrm{H} & -6.730758 & -1.705434 & -0.765873 \\ \mathrm{H} & -5.450865 & 0.406895 & -1.074258 \\ \mathrm{C} & -1.605179 & -1.271755 & 0.646598 \\ \mathrm{~N} & -2.757785 & 0.551166 & -0.304729 \\ \mathrm{C} & -1.505055 & 0.114797 & 0.215732 \\ \mathrm{C} & -0.526136 & 1.108511 & 0.170696 \\ \mathrm{H} & -2.631519 & 1.778885 & -0.668560 \\ \mathrm{O} & -0.751509 & -2.019475 & 1.159242 \\ \mathrm{C} & -0.842631 & 2.433506 & -0.355248 \\ \mathrm{~N} & 0.802834 & 1.111821 & 0.568203 \\ \mathrm{C} & 1.340192 & 2.365488 & 0.314992 \\ \mathrm{C} & 0.351149 & 3.211879 & -0.250970 \\ \mathrm{O} & -1.991639 & 2.791438 & -0.809424 \\ \mathrm{C} & 2.631835 & 2.839207 & 0.547574 \\ \mathrm{C} & 0.649754 & 4.540378 & -0.583217 \\ \mathrm{C} & 2.907260 & 4.162625 & 0.210470 \\ \mathrm{C} & 1.933019 & 5.009116 & -0.349315 \\ \mathrm{H} & 3.400614 & 2.208212 & 0.975037 \\ \mathrm{H} & 3.905322 & 4.549280 & 0.384384 \\ \mathrm{H} & 2.192536 & 6.031364 & -0.597111 \\ \mathrm{H} & -0.114106 & 5.177577 & -1.013296 \\ \mathrm{C} & 1.575119 & -0.018472 & 1.092407 \\ \mathrm{C} & 2.370417 & -0.754685 & 0.013387 \\ \mathrm{H} & 0.874619 & -0.709372 & 1.558018 \\ \mathrm{H} & 2.240384 & 0.373259 & 1.866092 \\ \mathrm{H} & 1.70116 & -1.183172 & -0.735661 \\ \mathrm{H} & 3.082585 & -0.098029 & -0.492947 \\ \mathrm{H} & 2.928315 & -1.576750 & 0.470605\end{array}$




\section{CC2/6-311G(d,p) geometries (in $\AA$ )}

$\begin{array}{lrrr}\mathrm{S}_{0} \text { Ind (isomer 1), gas phase } \\ & & & \\ & & & \\ \mathrm{C} & -2.9154267 & 0.6740748 & 0.0000216 \\ \mathrm{C} & -4.1613375 & 1.3081006 & -0.0002488 \\ \mathrm{C} & -5.3112789 & 0.5153821 & -0.0005107 \\ \mathrm{C} & -5.2051397 & -0.8900397 & -0.0005236 \\ \mathrm{C} & -3.9657821 & -1.5400364 & -0.0002178 \\ \mathrm{C} & -2.8197068 & -0.7386500 & 0.0000441 \\ \mathrm{H} & -4.2137447 & 2.3938889 & -0.0002002 \\ \mathrm{H} & -6.2934218 & 0.9781131 & -0.0007058 \\ \mathrm{H} & -6.1112080 & -1.4904801 & -0.0007750 \\ \mathrm{H} & -3.8993569 & -2.6245130 & -0.0002282 \\ \mathrm{C} & -1.5482216 & 1.2113854 & 0.0002397 \\ \mathrm{~N} & -1.4838879 & -1.1185483 & 0.0003543 \\ \mathrm{C} & -0.6856805 & 0.0008214 & 0.0003399 \\ \mathrm{C} & 0.6856488 & -0.0007831 & 0.0002648 \\ \mathrm{H} & -1.0964403 & -2.0561566 & 0.0005091 \\ \mathrm{O} & -1.1357998 & 2.3853223 & 0.0003278 \\ \mathrm{C} & 1.5481900 & -1.2113471 & 0.0001272 \\ \mathrm{~N} & 1.4838565 & 1.1185866 & 0.0002388 \\ \mathrm{H} & 1.0964095 & 2.0561951 & -0.0001183 \\ \mathrm{C} & 2.8196755 & 0.7386881 & -0.0001684 \\ \mathrm{C} & 2.9153951 & -0.6740368 & -0.0002004 \\ \mathrm{O} & 1.1357680 & -2.3852842 & 0.0002438 \\ \mathrm{C} & 3.9657509 & 1.5400746 & -0.0003819 \\ \mathrm{C} & 4.1613058 & -1.3080629 & -0.0003893 \\ \mathrm{C} & 5.2051083 & 0.8900775 & -0.0005106 \\ \mathrm{C} & 5.3112470 & -0.5153443 & -0.0005428 \\ \mathrm{H} & 3.8993261 & 2.6245512 & -0.0004947 \\ \mathrm{H} & 6.1111768 & 1.4905175 & -0.0005614 \\ \mathrm{H} & 6.2933898 & -0.9780755 & -0.0006715 \\ \mathrm{H} & 4.2137129 & -2.3938512 & -0.0004270\end{array}$


$\mathrm{S}_{1}$ Ind (isomer 1), gas phase

$\begin{array}{rrrr}\mathrm{C} & -2.9038855 & 0.6929436 & -0.0002031 \\ \mathrm{C} & -4.1575779 & 1.3070508 & -0.0002195 \\ \mathrm{C} & -5.3040941 & 0.4962316 & -0.0001089 \\ \mathrm{C} & -5.2053429 & -0.9081067 & -0.0000152 \\ \mathrm{C} & -3.9543844 & -1.5465735 & -0.0000230 \\ \mathrm{C} & -2.8248941 & -0.7302409 & -0.0001030 \\ \mathrm{H} & -4.2291065 & 2.3917932 & -0.0003373 \\ \mathrm{H} & -6.2873475 & 0.9585685 & -0.0000818 \\ \mathrm{H} & -6.1092717 & -1.5105422 & 0.0000621 \\ \mathrm{H} & -3.8714700 & -2.6304556 & 0.0000348 \\ \mathrm{C} & -1.5368373 & 1.2328359 & -0.0002566 \\ \mathrm{~N} & -1.4816644 & -1.0909769 & -0.0001207 \\ \mathrm{C} & -0.6953218 & 0.0318811 & -0.0002183 \\ \mathrm{C} & 0.6879540 & -0.0464570 & -0.0001776 \\ \mathrm{H} & -1.0508906 & -2.0211211 & -0.0000744 \\ \mathrm{O} & -1.1154167 & 2.4206435 & -0.0003135 \\ \mathrm{C} & 1.5294508 & -1.2474284 & -0.0001171 \\ \mathrm{~N} & 1.4743150 & 1.0763834 & -0.0002592 \\ \mathrm{H} & 1.0435748 & 2.0065395 & -0.0002994 \\ \mathrm{C} & 2.8175412 & 0.7156219 & -0.0002630 \\ \mathrm{C} & 2.8965068 & -0.7075632 & -0.0001637 \\ \mathrm{O} & 1.1080048 & -2.4352287 & -0.0000387 \\ \mathrm{C} & 3.9470457 & 1.5319325 & -0.0003160 \\ \mathrm{C} & 4.1501883 & -1.3216944 & -0.0001291 \\ \mathrm{C} & 5.1979932 & 0.8934423 & -0.0003023 \\ \mathrm{C} & 5.2967193 & -0.5108972 & -0.0001856 \\ \mathrm{H} & 3.8641526 & 2.6158163 & -0.0003669 \\ \mathrm{H} & 6.1019325 & 1.4958620 & -0.0003877 \\ \mathrm{H} & 6.2799642 & -0.9732522 & -0.0001358 \\ \mathrm{H} & 4.2216960 & -2.4064382 & -0.0000484\end{array}$


$\mathrm{S}_{0}$ Ind (isomer 2), gas phase

$\begin{array}{rrrr}\mathrm{C} & -2.9465918 & 0.7035990 & -0.0001011 \\ \mathrm{C} & -4.2144153 & 1.2784044 & -0.0001249 \\ \mathrm{C} & -5.3244361 & 0.4193749 & -0.0000819 \\ \mathrm{C} & -5.1415135 & -0.9731819 & -0.0000433 \\ \mathrm{C} & -3.8600380 & -1.5522925 & 0.0001109 \\ \mathrm{C} & -2.7614120 & -0.6963782 & -0.0000084 \\ \mathrm{H} & -4.3298703 & 2.3598498 & -0.0001995 \\ \mathrm{H} & -6.3300968 & 0.8290651 & -0.0000348 \\ \mathrm{H} & -6.0139732 & -1.6216750 & -0.0001746 \\ \mathrm{H} & -3.7231288 & -2.6299219 & 0.0004003 \\ \mathrm{C} & -1.5934626 & 1.2924600 & -0.0003059 \\ \mathrm{~N} & -1.3929794 & -1.0768143 & -0.0003031 \\ \mathrm{C} & -0.6976947 & 0.0593512 & -0.0003427 \\ \mathrm{C} & 0.7051745 & 0.0750248 & -0.0003177 \\ \mathrm{O} & -1.2164544 & 2.4649991 & -0.0004363 \\ \mathrm{C} & 1.5082027 & -1.0870351 & -0.0003699 \\ \mathrm{~N} & 1.5323035 & 1.1885333 & -0.0000789 \\ \mathrm{H} & 1.1839933 & 2.1380326 & -0.0000297 \\ \mathrm{C} & 2.8396969 & 0.7680010 & -0.0000230 \\ \mathrm{C} & 2.8652759 & -0.6651025 & -0.0001311 \\ \mathrm{O} & 1.0367697 & -2.3373570 & -0.0006778 \\ \mathrm{C} & 4.0263178 & 1.5165197 & -0.0001143 \\ \mathrm{C} & 4.0978618 & -1.3491544 & -0.0002813 \\ \mathrm{C} & 5.2297344 & 0.8149495 & -0.0000477 \\ \mathrm{C} & 5.2688475 & -0.6015869 & -0.0000763 \\ \mathrm{H} & 4.0071167 & 2.6031380 & -0.0003114 \\ \mathrm{H} & 6.1635969 & 1.3708333 & 0.0000756 \\ \mathrm{H} & 6.2309913 & -1.1053308 & 0.0002132 \\ \mathrm{H} & 4.1182545 & -2.4354455 & -0.0006989 \\ \mathrm{H} & 0.0485661 & -2.2385117 & -0.0008522\end{array}$


$\mathrm{S}_{1}$ Ind (isomer 2), gas phase

$\begin{array}{lrrr}\mathrm{C} & -2.8893321 & 0.7339691 & 0.0005595 \\ \mathrm{C} & -4.1808125 & 1.2670817 & 0.0000713 \\ \mathrm{C} & -5.2704382 & 0.3865055 & -0.0007300 \\ \mathrm{C} & -5.0885941 & -1.0273400 & -0.0010187 \\ \mathrm{C} & -3.8210156 & -1.5877596 & -0.0004047 \\ \mathrm{C} & -2.6961459 & -0.7131705 & 0.0000844 \\ \mathrm{H} & -4.3247311 & 2.3451526 & 0.0004948 \\ \mathrm{H} & -6.2823083 & 0.7840691 & -0.0011577 \\ \mathrm{H} & -5.9636731 & -1.6717245 & -0.0016369 \\ \mathrm{H} & -3.6649823 & -2.6637069 & -0.0005155 \\ \mathrm{C} & -1.5674037 & 1.3396457 & 0.0013393 \\ \mathrm{~N} & -1.4024250 & -1.0743806 & 0.0008060 \\ \mathrm{C} & -0.7089673 & 0.1710985 & 0.0015455 \\ \mathrm{C} & 0.6765521 & 0.1210470 & -0.0007426 \\ \mathrm{O} & -1.1730818 & 2.5504619 & 0.0012717 \\ \mathrm{C} & 1.4721042 & -1.0716153 & -0.0002569 \\ \mathrm{~N} & 1.5156746 & 1.2127468 & -0.0005945 \\ \mathrm{H} & 1.1403320 & 2.1582615 & -0.0004370 \\ \mathrm{C} & 2.8187879 & 0.7758220 & -0.0001901 \\ \mathrm{C} & 2.8297119 & -0.6600576 & -0.0003155 \\ \mathrm{O} & 0.9935789 & -2.3060100 & -0.0000225 \\ \mathrm{C} & 4.0132498 & 1.5067266 & -0.0006444 \\ \mathrm{C} & 4.0526396 & -1.3631003 & -0.0001598 \\ \mathrm{C} & 5.2080067 & 0.7861630 & -0.0005576 \\ \mathrm{C} & 5.2327706 & -0.6306060 & -0.0003859 \\ \mathrm{H} & 4.0088540 & 2.5930430 & -0.0007812 \\ \mathrm{H} & 6.1487192 & 1.3306281 & -0.0009439 \\ \mathrm{H} & 6.1891401 & -1.1446702 & -0.0003181 \\ \mathrm{H} & 4.0567366 & -2.4495939 & 0.0000196 \\ \mathrm{H} & -0.0063106 & -2.1863388 & 0.0002542\end{array}$


$\mathrm{S}_{0}$ Ind $\left(\mathrm{TS}_{12}\right)$, gas phase

$\begin{array}{lrrr}\mathrm{C} & 2.9603523 & 0.6766309 & 0.0017752 \\ \mathrm{C} & 4.2683974 & 1.1580647 & -0.0125925 \\ \mathrm{C} & 5.3152837 & 0.2265311 & -0.0204495 \\ \mathrm{C} & 5.0375529 & -1.1517102 & -0.0134647 \\ \mathrm{C} & 3.7225518 & -1.6433203 & 0.0009671 \\ \mathrm{C} & 2.6851738 & -0.7113406 & 0.0091095 \\ \mathrm{H} & 4.4563289 & 2.2291348 & -0.0179957 \\ \mathrm{H} & 6.3466106 & 0.5660423 & -0.0316389 \\ \mathrm{H} & 5.8632791 & -1.8586830 & -0.0198922 \\ \mathrm{H} & 3.5173189 & -2.7100399 & 0.0064641 \\ \mathrm{C} & 1.6588498 & 1.3755385 & 0.0106685 \\ \mathrm{~N} & 1.3004427 & -0.9595105 & 0.0248239 \\ \mathrm{C} & 0.6731528 & 0.2375696 & 0.0233793 \\ \mathrm{C} & -0.7150049 & 0.2469688 & 0.0334456 \\ \mathrm{H} & 0.3623999 & -1.8247072 & 0.0212624 \\ \mathrm{O} & 1.3805301 & 2.5774288 & 0.0074950 \\ \mathrm{C} & -1.4221723 & -1.0042916 & 0.0203372 \\ \mathrm{~N} & -1.6211205 & 1.2850317 & 0.0307407 \\ \mathrm{H} & -1.3636225 & 2.2619560 & 0.0106279 \\ \mathrm{C} & -2.8981550 & 0.7552792 & 0.0081891 \\ \mathrm{C} & -2.8208421 & -0.6707135 & 0.0096716 \\ \mathrm{O} & -0.8136298 & -2.1522032 & 0.0185005 \\ \mathrm{C} & -4.1346955 & 1.4137599 & 0.0026285 \\ \mathrm{C} & -3.9941235 & -1.4438789 & -0.0109944 \\ \mathrm{C} & -5.2855126 & 0.6246971 & -0.0192096 \\ \mathrm{C} & -5.2217210 & -0.7877689 & -0.0206318 \\ \mathrm{H} & -4.1970992 & 2.4987735 & 0.0044288 \\ \mathrm{H} & -6.2568943 & 1.1121774 & -0.0266315 \\ \mathrm{H} & -6.1432003 & -1.3620879 & -0.0366723 \\ \mathrm{H} & -3.9282018 & -2.5285693 & -0.0115756\end{array}$


$\mathrm{S}_{1}$ Ind $\left(\mathrm{TS}_{12}\right)$, gas phase

$\begin{array}{lrrr}\mathrm{C} & 2.9286213 & 0.6992811 & 0.0199133 \\ \mathrm{C} & 4.2469055 & 1.1412838 & -0.0159708 \\ \mathrm{C} & 5.2754396 & 0.1828381 & -0.0407469 \\ \mathrm{C} & 5.0001006 & -1.2076622 & -0.0356688 \\ \mathrm{C} & 3.6907059 & -1.6785867 & 0.0009167 \\ \mathrm{C} & 2.6545320 & -0.7168712 & 0.0210150 \\ \mathrm{H} & 4.4628310 & 2.2069134 & -0.0183059 \\ \mathrm{H} & 6.3111829 & 0.5116871 & -0.0666995 \\ \mathrm{H} & 5.8254106 & -1.9136206 & -0.0544261 \\ \mathrm{H} & 3.4610764 & -2.7410143 & 0.0061703 \\ \mathrm{C} & 1.6410530 & 1.4301534 & 0.0481442 \\ \mathrm{~N} & 1.3216704 & -0.9239240 & 0.0596431 \\ \mathrm{C} & 0.6794368 & 0.3555396 & 0.0826015 \\ \mathrm{C} & -0.6995649 & 0.2675428 & 0.0160637 \\ \mathrm{H} & 0.4801935 & -1.7676032 & 0.0475778 \\ \mathrm{O} & 1.4203244 & 2.6691396 & 0.0354644 \\ \mathrm{C} & -1.4008594 & -1.0154327 & 0.0171864 \\ \mathrm{~N} & -1.6187507 & 1.2808207 & 0.0124190 \\ \mathrm{H} & -1.3575765 & 2.2595681 & 0.0174417 \\ \mathrm{C} & -2.9012659 & 0.7417986 & 0.0035036 \\ \mathrm{C} & -2.8045666 & -0.6804876 & 0.0019138 \\ \mathrm{O} & -0.8133111 & -2.1649296 & 0.0245708 \\ \mathrm{C} & -4.1363381 & 1.3884284 & -0.0220161 \\ \mathrm{C} & -3.9670563 & -1.4655783 & -0.0062734 \\ \mathrm{C} & -5.2857621 & 0.5859019 & -0.0272447 \\ \mathrm{C} & -5.2058772 & -0.8215864 & -0.0239609 \\ \mathrm{H} & -4.2085352 & 2.4728815 & -0.0257522 \\ \mathrm{H} & -6.2613739 & 1.0646721 & -0.0427959 \\ \mathrm{H} & -6.1202599 & -1.4076247 & -0.0298732 \\ \mathrm{H} & -3.8879518 & -2.5496151 & -0.0056301\end{array}$


$\mathrm{S}_{0}$ NHxInd (isomer 1), gas phase

$\begin{array}{lrrr}\mathrm{C} & 2.9249039 & -1.7057164 & -0.2086699 \\ \mathrm{C} & 3.4937324 & -2.9816882 & -0.2370729 \\ \mathrm{C} & 4.8444852 & -3.1096543 & 0.0964640 \\ \mathrm{C} & 5.6029995 & -1.9715895 & 0.4384024 \\ \mathrm{C} & 5.0461286 & -0.6873832 & 0.4577137 \\ \mathrm{C} & 3.6921271 & -0.5730861 & 0.1264905 \\ \mathrm{H} & 2.8819730 & -3.8403526 & -0.5022096 \\ \mathrm{H} & 5.3181635 & -4.0866308 & 0.0912540 \\ \mathrm{H} & 6.6523878 & -2.0917094 & 0.6953023 \\ \mathrm{H} & 5.6434116 & 0.1827662 & 0.7156643 \\ \mathrm{C} & 1.5554078 & -1.2616882 & -0.5012143 \\ \mathrm{~N} & 2.9044021 & 0.5628591 & 0.0362851 \\ \mathrm{C} & 1.6027649 & 0.2338579 & -0.2935335 \\ \mathrm{C} & 0.6237333 & 1.2201546 & -0.2639164 \\ \mathrm{H} & 3.1066857 & 1.4996534 & 0.3939554 \\ \mathrm{O} & 0.5834270 & -1.9635290 & -0.8146415 \\ \mathrm{C} & 0.9185245 & 2.5778749 & 0.2955247 \\ \mathrm{~N} & -0.7162748 & 1.1767978 & -0.6015241 \\ \mathrm{C} & -1.3125239 & 2.3921522 & -0.2721193 \\ \mathrm{C} & -0.3651902 & 3.2708584 & 0.2947169 \\ \mathrm{O} & 2.0305918 & 2.9935540 & 0.6841150 \\ \mathrm{C} & -2.6440580 & 2.7910014 & -0.4525982 \\ \mathrm{C} & -0.7143957 & 4.5700883 & 0.6730415 \\ \mathrm{C} & -2.9836670 & 4.0909762 & -0.0596369 \\ \mathrm{C} & -2.0410083 & 4.9731207 & 0.5061217 \\ \mathrm{H} & -3.3905028 & 2.1301459 & -0.8819296 \\ \mathrm{H} & -4.0114673 & 4.4222411 & -0.1849923 \\ \mathrm{H} & -2.3501467 & 5.9720801 & 0.7982762 \\ \mathrm{H} & 0.0385355 & 5.2293645 & 1.0974176 \\ \mathrm{C} & -1.4416756 & 0.1303277 & -1.3160963 \\ \mathrm{C} & -2.2736974 & -0.7348559 & -0.3792795 \\ \mathrm{H} & -0.7115989 & -0.4819302 & -1.8423943 \\ \mathrm{H} & -2.0742664 & 0.6344777 & -2.0562192 \\ \mathrm{H} & -1.6135723 & -1.2673676 & 0.3072851 \\ \mathrm{H} & -2.9800947 & -0.1272679 & 0.1937771 \\ \mathrm{H} & -2.8355684 & -1.4751262 & -0.9580346\end{array}$


$\mathrm{S}_{1}$ NHxInd (isomer 1), gas phase

$\begin{array}{lrrc}\mathrm{C} & 2.9006045 & -1.7113226 & -0.3365828 \\ \mathrm{C} & 3.5348866 & -2.9463535 & -0.4694648 \\ \mathrm{C} & 4.8767374 & -3.0520469 & -0.0697684 \\ \mathrm{C} & 5.5686381 & -1.9476579 & 0.4669079 \\ \mathrm{C} & 4.9458715 & -0.6984996 & 0.6053393 \\ \mathrm{C} & 3.6098653 & -0.6105194 & 0.2078932 \\ \mathrm{H} & 2.9932447 & -3.7922665 & -0.8853916 \\ \mathrm{H} & 5.3925292 & -4.0033873 & -0.1677201 \\ \mathrm{H} & 6.6063992 & -2.0606759 & 0.7679700 \\ \mathrm{H} & 5.4729055 & 0.1575135 & 1.0188196 \\ \mathrm{C} & 1.5276159 & -1.2872257 & -0.6505116 \\ \mathrm{~N} & 2.7633748 & 0.4794464 & 0.2208189 \\ \mathrm{C} & 1.5099757 & 0.1479816 & -0.2630301 \\ \mathrm{C} & 0.5676147 & 1.1884549 & -0.1852585 \\ \mathrm{H} & 2.8823097 & 1.4515832 & 0.5996224 \\ \mathrm{O} & 0.6053997 & -1.9919466 & -1.1303591 \\ \mathrm{C} & 0.8741544 & 2.5060028 & 0.4032581 \\ \mathrm{~N} & -0.7333168 & 1.2043761 & -0.6542403 \\ \mathrm{C} & -1.3193806 & 2.4427155 & -0.3653430 \\ \mathrm{C} & -0.3551537 & 3.2754329 & 0.2550416 \\ \mathrm{O} & 1.9754731 & 2.8732504 & 0.9168583 \\ \mathrm{C} & -2.6159954 & 2.8800007 & -0.6353639 \\ \mathrm{C} & -0.6989992 & 4.5709044 & 0.6466980 \\ \mathrm{C} & -2.9455267 & 4.1880632 & -0.2415135 \\ \mathrm{C} & -1.9986867 & 5.0248103 & 0.3782700 \\ \mathrm{H} & -3.3572865 & 2.2387646 & -1.1032310 \\ \mathrm{H} & -3.9474651 & 4.5613769 & -0.4346008 \\ \mathrm{H} & -2.2880894 & 6.0310269 & 0.6684998 \\ \mathrm{H} & 0.0401551 & 5.2051088 & 1.1294014 \\ \mathrm{C} & -1.4699872 & 0.081129 & -1.2221086 \\ \mathrm{C} & -2.1178756 & -0.7576078 & -0.1252442 \\ \mathrm{H} & -0.7627236 & -0.5377770 & -1.7787422 \\ \mathrm{H} & -2.2148447 & 0.5041899 & -1.9022696 \\ \mathrm{H} & -1.3436881 & -1.1724077 & 0.5232079 \\ \mathrm{H} & -2.8131956 & -0.1562734 & 0.4679412 \\ \mathrm{H} & -2.6608622 & -1.5913684 & -0.5800764\end{array}$


$\mathrm{S}_{0}$ NHxInd (isomer 2), gas phase

$\begin{array}{lrrr}\mathrm{C} & 3.0075030 & -1.6386725 & -0.5590821 \\ \mathrm{C} & 3.7172681 & -2.7777392 & -0.9288545 \\ \mathrm{C} & 4.9943495 & -2.9595735 & -0.3766310 \\ \mathrm{C} & 5.5272612 & -2.0045306 & 0.5059344 \\ \mathrm{C} & 4.8084473 & -0.8544236 & 0.8749845 \\ \mathrm{C} & 3.5419305 & -0.6803332 & 0.3199435 \\ \mathrm{H} & 3.2821216 & -3.4989265 & -1.6168864 \\ \mathrm{H} & 5.5814022 & -3.8348174 & -0.6384929 \\ \mathrm{H} & 6.5190569 & -2.1634828 & 0.9216466 \\ \mathrm{H} & 5.2239207 & -0.1193532 & 1.5586191 \\ \mathrm{C} & 1.6761675 & -1.1479135 & -0.9621138 \\ \mathrm{~N} & 2.6529477 & 0.3901131 & 0.5549606 \\ \mathrm{C} & 1.5384313 & 0.1613221 & -0.1573576 \\ \mathrm{C} & 0.5194655 & 1.1422816 & -0.1405818 \\ \mathrm{O} & 0.8930490 & -1.6433384 & -1.7692394 \\ \mathrm{C} & 0.7697639 & 2.4282613 & 0.4228282 \\ \mathrm{~N} & -0.7426129 & 1.1585365 & -0.7481897 \\ \mathrm{C} & -1.3012753 & 2.4043616 & -0.5115509 \\ \mathrm{C} & -0.3722139 & 3.2365298 & 0.1799271 \\ \mathrm{O} & 1.8944632 & 2.7998042 & 1.0207079 \\ \mathrm{C} & -2.5840411 & 2.8770477 & -0.8326347 \\ \mathrm{C} & -0.6883928 & 4.5750218 & 0.4806568 \\ \mathrm{C} & -2.8820447 & 4.2026048 & -0.5174253 \\ \mathrm{C} & -1.9505572 & 5.0447218 & 0.1365006 \\ \mathrm{H} & -3.3141232 & 2.2539008 & -1.3402164 \\ \mathrm{H} & -3.8653623 & 4.5930594 & -0.7668059 \\ \mathrm{H} & -2.2259183 & 6.0718252 & 0.3575343 \\ \mathrm{H} & 0.0342933 & 5.2048793 & 0.9922465 \\ \mathrm{C} & -1.5842706 & -0.0263457 & -0.9281946 \\ \mathrm{H} & -0.9988429 & -0.7738146 & -1.4617707 \\ \mathrm{H} & -2.4136878 & 0.2728723 & -1.5747229 \\ \mathrm{H} & 2.4565319 & 1.9560648 & 1.0218163 \\ \mathrm{C} & -2.0957316 & -0.5624446 & 0.4039768 \\ \mathrm{H} & -2.6721329 & 0.2028669 & 0.9323757 \\ \mathrm{H} & -1.2574575 & -0.8661418 & 1.0379046 \\ \mathrm{H} & -2.7369157 & -1.4344766 & 0.2402403\end{array}$


$\mathrm{S}_{1}$ NHxInd (isomer 2), gas phase

$\begin{array}{lrrc}\mathrm{C} & 2.9088671 & -1.7323698 & -0.2869980 \\ \mathrm{C} & 3.5576992 & -2.9633924 & -0.3894251 \\ \mathrm{C} & 4.9274540 & -3.0164148 & -0.0823941 \\ \mathrm{C} & 5.6310563 & -1.8685611 & 0.3341776 \\ \mathrm{C} & 4.9846597 & -0.6284626 & 0.4421454 \\ \mathrm{C} & 3.6209529 & -0.5815867 & 0.1385552 \\ \mathrm{H} & 3.0118074 & -3.8475146 & -0.7108442 \\ \mathrm{H} & 5.4572213 & -3.9626903 & -0.1586142 \\ \mathrm{H} & 6.6906801 & -1.9439801 & 0.5631169 \\ \mathrm{H} & 5.5145312 & 0.2661434 & 0.7610334 \\ \mathrm{C} & 1.5162141 & -1.3293313 & -0.5103648 \\ \mathrm{~N} & 2.7849004 & 0.5341293 & 0.1777381 \\ \mathrm{C} & 1.5579299 & 0.1184472 & -0.2090231 \\ \mathrm{C} & 0.5649478 & 1.1968167 & -0.1482192 \\ \mathrm{O} & 0.5377538 & -2.0420123 & -0.8727737 \\ \mathrm{C} & 0.8362507 & 2.4721102 & 0.4136571 \\ \mathrm{~N} & -0.7208697 & 1.2009646 & -0.6194263 \\ \mathrm{C} & -1.3122899 & 2.4449002 & -0.3500501 \\ \mathrm{C} & -0.3437323 & 3.2751557 & 0.2733707 \\ \mathrm{O} & 1.9860668 & 2.8688788 & 0.9424260 \\ \mathrm{C} & -2.5960486 & 2.8924648 & -0.6447907 \\ \mathrm{C} & -0.6793766 & 4.5792898 & 0.6517517 \\ \mathrm{C} & -2.9181825 & 4.2065770 & -0.2640097 \\ \mathrm{C} & -1.9715650 & 5.0374463 & 0.3607241 \\ \mathrm{H} & -3.3345013 & 2.2547315 & -1.1218997 \\ \mathrm{H} & -3.9127751 & 4.5892576 & -0.4746629 \\ \mathrm{H} & -2.2548830 & 6.0483264 & 0.6400487 \\ \mathrm{H} & 0.0531525 & 5.2195917 & 1.1354053 \\ \mathrm{C} & -1.4556783 & 0.0794098 & -1.2078176 \\ \mathrm{H} & -0.7304691 & -0.5835645 & -1.6820011 \\ \mathrm{H} & -2.1247891 & 0.5085840 & -1.9596506 \\ \mathrm{H} & 2.5929415 & 2.0609614 & 0.7756539 \\ \mathrm{C} & -2.2195974 & -0.6903900 & -0.1376405 \\ \mathrm{H} & -2.9208760 & -0.0393954 & 0.3927887 \\ \mathrm{H} & -1.5116324 & -1.1279062 & 0.5683601 \\ \mathrm{H} & -2.7750263 & -1.5068666 & -0.6082943\end{array}$


$\mathrm{S}_{0}$ NHxInd $\left(\mathrm{TS}_{12}\right)$, gas phase

$\begin{array}{lrrr}\mathrm{C} & -3.0003839 & -1.6253020 & 0.3517407 \\ \mathrm{C} & -3.6937898 & -2.8217423 & 0.5266835 \\ \mathrm{C} & -5.0157645 & -2.8872086 & 0.0668752 \\ \mathrm{C} & -5.6153779 & -1.7634597 & -0.5303255 \\ \mathrm{C} & -4.9217898 & -0.5549392 & -0.7000374 \\ \mathrm{C} & -3.6049110 & -0.4999870 & -0.2417644 \\ \mathrm{H} & -3.2059416 & -3.6732804 & 0.9951966 \\ \mathrm{H} & -5.5876346 & -3.8032814 & 0.1803868 \\ \mathrm{H} & -6.6429414 & -1.8338608 & -0.8780742 \\ \mathrm{H} & -5.3934576 & 0.3093222 & -1.1592014 \\ \mathrm{C} & -1.6322156 & -1.2370461 & 0.7398171 \\ \mathrm{~N} & -2.7196917 & 0.5841056 & -0.2908654 \\ \mathrm{C} & -1.5391183 & 0.2127785 & 0.2646762 \\ \mathrm{C} & -0.5160675 & 1.1761435 & 0.2492600 \\ \mathrm{H} & -2.5731701 & 1.8099234 & -0.6862248 \\ \mathrm{O} & -0.7784072 & -1.9166482 & 1.3125838 \\ \mathrm{C} & -0.8018403 & 2.4710341 & -0.3305049 \\ \mathrm{~N} & 0.7844387 & 1.1839419 & 0.7352290 \\ \mathrm{C} & 1.3549546 & 2.4073314 & 0.4144349 \\ \mathrm{C} & 0.3959188 & 3.2456462 & -0.2220099 \\ \mathrm{O} & -1.9541789 & 2.8026439 & -0.8339299 \\ \mathrm{C} & 2.6648926 & 2.8588266 & 0.6380597 \\ \mathrm{C} & 0.7232209 & 4.5591413 & -0.5975115 \\ \mathrm{C} & 2.9735406 & 4.1636217 & 0.2490861 \\ \mathrm{C} & 2.0196613 & 5.0083348 & -0.3641394 \\ \mathrm{H} & 3.4172808 & 2.2304145 & 1.1049368 \\ \mathrm{H} & 3.9819430 & 4.5345186 & 0.4142418 \\ \mathrm{H} & 2.3047200 & 6.0173397 & -0.6469637 \\ \mathrm{H} & -0.0229840 & 5.1897704 & -1.0735250 \\ \mathrm{C} & 1.5690968 & 0.0116734 & 1.1106657 \\ \mathrm{C} & 2.0613427 & -0.7565337 & -0.1093002 \\ \mathrm{H} & 0.9436027 & -0.6261355 & 1.7340572 \\ \mathrm{H} & 2.4053596 & 0.3725422 & 1.7159881 \\ \mathrm{H} & 1.2105215 & -1.1165386 & -0.6937083 \\ \mathrm{H} & 2.6818779 & -0.1175185 & -0.7446562 \\ \mathrm{H} & 2.6505220 & -1.6239217 & 0.2044473\end{array}$

S64 (S169) 
$\mathrm{S}_{1}$ NHxInd $\left(\mathrm{TS}_{12}\right)$, gas phase

$\begin{array}{lrrr}\mathrm{C} & -2.9543913 & -1.6189119 & 0.3319655 \\ \mathrm{C} & -3.6530622 & -2.8185662 & 0.4359171 \\ \mathrm{C} & -4.9913889 & -2.8488145 & 0.0087513 \\ \mathrm{C} & -5.6268752 & -1.6993206 & -0.5203188 \\ \mathrm{C} & -4.9463154 & -0.4872541 & -0.6300150 \\ \mathrm{C} & -3.6013898 & -0.4591837 & -0.2047531 \\ \mathrm{H} & -3.1633448 & -3.6992317 & 0.8445035 \\ \mathrm{H} & -5.5565467 & -3.7742453 & 0.0836324 \\ \mathrm{H} & -6.6633463 & -1.7637166 & -0.8395148 \\ \mathrm{H} & -5.4212761 & 0.4034367 & -1.0335510 \\ \mathrm{C} & -1.5673407 & -1.2683417 & 0.6848943 \\ \mathrm{~N} & -2.7424419 & 0.5882218 & -0.2024430 \\ \mathrm{C} & -1.4904739 & 0.1475570 & 0.3192765 \\ \mathrm{C} & -0.5260629 & 1.1621716 & 0.2501946 \\ \mathrm{H} & -2.6355028 & 1.7525446 & -0.5871420 \\ \mathrm{O} & -0.6887105 & -2.0295797 & 1.1708173 \\ \mathrm{C} & -0.8399102 & 2.4766707 & -0.3203765 \\ \mathrm{~N} & 0.7823438 & 1.1734351 & 0.6744300 \\ \mathrm{C} & 1.3535170 & 2.4073404 & 0.3582650 \\ \mathrm{C} & 0.3686836 & 3.2506691 & -0.2247689 \\ \mathrm{O} & -1.9871472 & 2.8297277 & -0.8004411 \\ \mathrm{C} & 2.6579631 & 2.8546553 & 0.5720440 \\ \mathrm{C} & 0.6907749 & 4.5557997 & -0.6196215 \\ \mathrm{C} & 2.9639782 & 4.1645143 & 0.1718549 \\ \mathrm{C} & 1.9952318 & 5.0093043 & -0.4053352 \\ \mathrm{H} & 3.4205179 & 2.2161769 & 1.0082143 \\ \mathrm{H} & 3.9737620 & 4.5370328 & 0.3236569 \\ \mathrm{H} & 2.2734698 & 6.0167854 & -0.7005683 \\ \mathrm{H} & -0.0683911 & 5.1910122 & -1.0686320 \\ \mathrm{C} & 1.5350843 & 0.0170625 & 1.1513674 \\ \mathrm{C} & 2.1119917 & -0.7895849 & -0.0053306 \\ \mathrm{H} & 0.8503855 & -0.6102386 & 1.7261975 \\ \mathrm{H} & 2.3204560 & 0.3989256 & 1.8109054 \\ \mathrm{H} & 1.2976835 & -1.1799437 & -0.6200320 \\ \mathrm{H} & 2.7728048 & -0.1737138 & -0.6230782 \\ \mathrm{H} & 2.6785018 & -1.6407456 & 0.3846573\end{array}$


CARTESIAN COORDINATES FOR $S_{0}$ AND $S_{1}$ IRC GEOMETRIES

\section{B3LYP/6-311G $(\mathrm{d}, \mathrm{p})$ geometries (in $\AA$ )}

$\mathrm{S}_{0}$ Ind (IRC point 1 toward isomer 2), gas phase

$\begin{array}{lrrc}\mathrm{C} & 2.95484072 & 0.67027761 & -0.00000109 \\ \mathrm{C} & 4.25967372 & 1.14623961 & 0.00000591 \\ \mathrm{C} & 5.30403772 & 0.22149961 & -0.00004109 \\ \mathrm{C} & 5.02502572 & -1.14997839 & -0.00009209 \\ \mathrm{C} & 3.71602472 & -1.64035139 & -0.00010009 \\ \mathrm{C} & 2.67772372 & -0.71651039 & -0.00005309 \\ \mathrm{H} & 4.45029172 & 2.21369361 & 0.00004591 \\ \mathrm{H} & 6.33258572 & 0.56114561 & -0.00003709 \\ \mathrm{H} & 5.84844672 & -1.85584739 & -0.00012809 \\ \mathrm{H} & 3.51531972 & -2.70482939 & -0.00014009 \\ \mathrm{C} & 1.65703372 & 1.36698461 & 0.00003891 \\ \mathrm{~N} & 1.29813872 & -0.96328539 & -0.00005009 \\ \mathrm{C} & 0.66598072 & 0.22179061 & 0.00000291 \\ \mathrm{C} & -0.71411028 & 0.23992261 & 0.00001691 \\ \mathrm{H} & 0.33985972 & -1.84986439 & -0.00007609 \\ \mathrm{O} & 1.37211172 & 2.54972061 & 0.00008891 \\ \mathrm{C} & -1.42643328 & -1.00426939 & -0.00002609 \\ \mathrm{~N} & -1.62729328 & 1.28319561 & 0.00006691 \\ \mathrm{H} & -1.37230428 & 2.25724861 & 0.00010291 \\ \mathrm{C} & -2.89961928 & 0.75379461 & 0.00005691 \\ \mathrm{C} & -2.82285628 & -0.66979639 & -0.00000009 \\ \mathrm{O} & -0.82360728 & -2.14217939 & -0.00007709 \\ \mathrm{C} & -4.13665228 & 1.40342261 & 0.00009391 \\ \mathrm{C} & -3.99399928 & -1.44060939 & -0.00002109 \\ \mathrm{C} & -5.27921328 & 0.61531661 & 0.00007291 \\ \mathrm{C} & -5.21669728 & -0.79296239 & 0.00001591 \\ \mathrm{H} & -4.20317828 & 2.48512461 & 0.00013791 \\ \mathrm{H} & -6.24956728 & 1.09914161 & 0.00010091 \\ \mathrm{H} & -6.13585528 & -1.36607739 & 0.00000091 \\ \mathrm{H} & -3.92784628 & -2.52213439 & -0.00006509 \\ & & & \end{array}$


$\mathrm{S}_{0}$ Ind (IRC point 20 toward isomer 2), gas phase

$\begin{array}{lrrr}\mathrm{C} & 2.97071800 & 0.68701100 & 0.00000000 \\ \mathrm{C} & 4.27330300 & 1.15725900 & 0.00000600 \\ \mathrm{C} & 5.31254500 & 0.22231300 & -0.00004100 \\ \mathrm{C} & 5.03153900 & -1.14713100 & -0.00009200 \\ \mathrm{C} & 3.71883200 & -1.62904500 & -0.00009900 \\ \mathrm{C} & 2.69412600 & -0.69446100 & -0.00005300 \\ \mathrm{H} & 4.47444600 & 2.22280600 & 0.00004600 \\ \mathrm{H} & 6.34237400 & 0.55815100 & -0.00003700 \\ \mathrm{H} & 5.85348200 & -1.85463100 & -0.00012800 \\ \mathrm{H} & 3.50341000 & -2.69063300 & -0.00013900 \\ \mathrm{C} & 1.66306600 & 1.37269400 & 0.00003900 \\ \mathrm{~N} & 1.31992100 & -0.95671500 & -0.00005000 \\ \mathrm{C} & 0.68156900 & 0.20310800 & 0.00000200 \\ \mathrm{C} & -0.71695600 & 0.22143200 & 0.00001600 \\ \mathrm{H} & 0.12017700 & -2.02157100 & -0.00008100 \\ \mathrm{O} & 1.37001300 & 2.55016700 & 0.00008900 \\ \mathrm{C} & -1.43995400 & -0.99005700 & -0.00002600 \\ \mathrm{~N} & -1.62460400 & 1.27746000 & 0.00006700 \\ \mathrm{H} & -1.36099900 & 2.24919800 & 0.00010200 \\ \mathrm{C} & -2.89918400 & 0.75980000 & 0.00005700 \\ \mathrm{C} & -2.82355100 & -0.66494500 & 0.00000000 \\ \mathrm{O} & -0.88188700 & -2.18331900 & -0.00007800 \\ \mathrm{C} & -4.13890600 & 1.40714000 & 0.00009400 \\ \mathrm{C} & -3.99477600 & -1.43992000 & -0.00002100 \\ \mathrm{C} & -5.27926800 & 0.61798400 & 0.00007300 \\ \mathrm{C} & -5.21484400 & -0.79236000 & 0.00001600 \\ \mathrm{H} & -4.20775700 & 2.48874000 & 0.00013800 \\ \mathrm{H} & -6.25047000 & 1.09989800 & 0.00010100 \\ \mathrm{H} & -6.13423600 & -1.36533400 & 0.00000100 \\ \mathrm{H} & -3.92816100 & -2.52123900 & -0.00006500\end{array}$


$\mathrm{S}_{0}$ Ind (IRC point 40 toward isomer 2), gas phase

$\begin{array}{lrrr}\mathrm{C} & 2.98041000 & 0.69810100 & 0.00000000 \\ \mathrm{C} & 4.28241800 & 1.17719400 & 0.00000700 \\ \mathrm{C} & 5.32972000 & 0.24969500 & -0.00004000 \\ \mathrm{C} & 5.05874500 & -1.12150500 & -0.00009200 \\ \mathrm{C} & 3.74848700 & -1.61034000 & -0.00009900 \\ \mathrm{C} & 2.71514400 & -0.68573000 & -0.00005200 \\ \mathrm{H} & 4.47817200 & 2.24381500 & 0.00004700 \\ \mathrm{H} & 6.35694200 & 0.59363600 & -0.00003600 \\ \mathrm{H} & 5.88472300 & -1.82428800 & -0.00012700 \\ \mathrm{H} & 3.53922000 & -2.67315600 & -0.00013900 \\ \mathrm{C} & 1.66062800 & 1.36613500 & 0.00003900 \\ \mathrm{~N} & 1.34022100 & -0.97203100 & -0.00005100 \\ \mathrm{C} & 0.68945200 & 0.17589900 & 0.00000100 \\ \mathrm{C} & -0.71562800 & 0.18646500 & 0.00001500 \\ \mathrm{H} & 0.04295800 & -2.11302900 & -0.00008400 \\ \mathrm{O} & 1.35276500 & 2.53930200 & 0.00008900 \\ \mathrm{C} & -1.46217600 & -1.00798800 & -0.00002600 \\ \mathrm{~N} & -1.60979100 & 1.25789400 & 0.00006600 \\ \mathrm{H} & -1.32696100 & 2.22442000 & 0.00010100 \\ \mathrm{C} & -2.89365600 & 0.76418600 & 0.00005700 \\ \mathrm{C} & -2.83732100 & -0.66057400 & 0.00000000 \\ \mathrm{O} & -0.95018000 & -2.22885100 & -0.00007900 \\ \mathrm{C} & -4.12927800 & 1.42230800 & 0.00009500 \\ \mathrm{C} & -4.01377500 & -1.42703800 & -0.00002000 \\ \mathrm{C} & -5.27649200 & 0.64222700 & 0.00007400 \\ \mathrm{C} & -5.22723800 & -0.76923800 & 0.00001700 \\ \mathrm{H} & -4.19108900 & 2.50433300 & 0.00013800 \\ \mathrm{H} & -6.24308100 & 1.13338300 & 0.00010200 \\ \mathrm{H} & -6.15242000 & -1.33284400 & 0.00000300 \\ \mathrm{H} & -3.95403300 & -2.50871400 & -0.00006400\end{array}$


$\mathrm{S}_{0}$ Ind (IRC point 60 toward isomer 2), gas phase

$\begin{array}{lrrr}\mathrm{C} & 2.97427400 & 0.69764300 & 0.00000000 \\ \mathrm{C} & 4.26718200 & 1.20053400 & 0.00000800 \\ \mathrm{C} & 5.33094900 & 0.29226000 & -0.00003800 \\ \mathrm{C} & 5.08276400 & -1.08310000 & -0.00009000 \\ \mathrm{C} & 3.78068600 & -1.59533000 & -0.00009800 \\ \mathrm{C} & 2.72982100 & -0.69041700 & -0.00005300 \\ \mathrm{H} & 4.44273900 & 2.27067000 & 0.00004800 \\ \mathrm{H} & 6.35208900 & 0.65386200 & -0.00003400 \\ \mathrm{H} & 5.92046900 & -1.77188300 & -0.00012600 \\ \mathrm{H} & 3.59171800 & -2.66194900 & -0.00013900 \\ \mathrm{C} & 1.64552800 & 1.34430500 & 0.00003800 \\ \mathrm{~N} & 1.35636700 & -0.99936300 & -0.00005200 \\ \mathrm{C} & 0.69291700 & 0.13990600 & 0.00000000 \\ \mathrm{C} & -0.71253700 & 0.15169500 & 0.00001300 \\ \mathrm{H} & 0.01263900 & -2.16258800 & -0.00008600 \\ \mathrm{O} & 1.31880700 & 2.51270500 & 0.00008800 \\ \mathrm{C} & -1.47496200 & -1.03089900 & -0.00002700 \\ \mathrm{~N} & -1.59019900 & 1.23616800 & 0.00006500 \\ \mathrm{H} & -1.28992100 & 2.19765400 & 0.00010000 \\ \mathrm{C} & -2.88032600 & 0.76271700 & 0.00005700 \\ \mathrm{C} & -2.84589600 & -0.66345200 & 0.00000000 \\ \mathrm{O} & -0.97809800 & -2.26058500 & -0.00008100 \\ \mathrm{C} & -4.10411800 & 1.44280400 & 0.00009500 \\ \mathrm{C} & -4.03714500 & -1.40802200 & -0.00001900 \\ \mathrm{C} & -5.26468100 & 0.68363400 & 0.00007500 \\ \mathrm{C} & -5.23887100 & -0.72864300 & 0.00001900 \\ \mathrm{H} & -4.14570200 & 2.52579600 & 0.00013900 \\ \mathrm{H} & -6.22274400 & 1.19120400 & 0.00010400 \\ \mathrm{H} & -6.17351700 & -1.27644800 & 0.00000500 \\ \mathrm{H} & -3.99821500 & -2.49066200 & -0.00006300\end{array}$


$\mathrm{S}_{0}$ Ind (IRC point 80 toward isomer 2 ), gas phase

$\begin{array}{lrrr}\mathrm{C} & 2.96566400 & 0.69681000 & 0.00000000 \\ \mathrm{C} & 4.24874600 & 1.22344800 & 0.00000900 \\ \mathrm{C} & 5.32848000 & 0.33463800 & -0.00003700 \\ \mathrm{C} & 5.10351300 & -1.04460300 & -0.00008900 \\ \mathrm{C} & 3.81034000 & -1.58045800 & -0.00009800 \\ \mathrm{C} & 2.74266300 & -0.69539000 & -0.00005300 \\ \mathrm{H} & 4.40398700 & 2.29671700 & 0.00005000 \\ \mathrm{H} & 6.34321300 & 0.71382900 & -0.00003100 \\ \mathrm{H} & 5.95298700 & -1.71883200 & -0.00012400 \\ \mathrm{H} & 3.64165000 & -2.65047000 & -0.00013900 \\ \mathrm{C} & 1.62842000 & 1.32153400 & 0.00003800 \\ \mathrm{~N} & 1.37179700 & -1.02698600 & -0.00005300 \\ \mathrm{C} & 0.69590600 & 0.10372000 & -0.00000200 \\ \mathrm{C} & -0.70884700 & 0.11747400 & 0.00001200 \\ \mathrm{H} & -0.01356900 & -2.20796600 & -0.00008700 \\ \mathrm{O} & 1.28125700 & 2.48451800 & 0.00008700 \\ \mathrm{C} & -1.48608700 & -1.05364200 & -0.00002800 \\ \mathrm{~N} & -1.56926200 & 1.21461900 & 0.00006300 \\ \mathrm{H} & -1.25134600 & 2.17068400 & 0.00009900 \\ \mathrm{C} & -2.86494100 & 0.76108000 & 0.00005700 \\ \mathrm{C} & -2.85240500 & -0.66625900 & 0.00000000 \\ \mathrm{O} & -1.00249700 & -2.29069200 & -0.00008200 \\ \mathrm{C} & -4.07603200 & 1.46326400 & 0.00009600 \\ \mathrm{C} & -4.05793300 & -1.38848200 & -0.00001800 \\ \mathrm{C} & -5.24953100 & 0.72532500 & 0.00007700 \\ \mathrm{C} & -5.24730100 & -0.68736400 & 0.00002100 \\ \mathrm{H} & -4.09698300 & 2.54684600 & 0.00013900 \\ \mathrm{H} & -6.19878800 & 1.24915300 & 0.00010600 \\ \mathrm{H} & -6.19126600 & -1.21897500 & 0.00000800 \\ \mathrm{H} & -4.04003100 & -2.47168700 & -0.00006200\end{array}$


$\mathrm{S}_{0}$ Ind (IRC point 100 toward isomer 2), gas phase

$\begin{array}{lrrr}\mathrm{C} & 2.95696700 & 0.69611700 & 0.00000000 \\ \mathrm{C} & 4.22984700 & 1.24619600 & 0.00001000 \\ \mathrm{C} & 5.32507600 & 0.37698300 & -0.00003500 \\ \mathrm{C} & 5.12329800 & -1.00575100 & -0.00008800 \\ \mathrm{C} & 3.83942700 & -1.56491700 & -0.00009800 \\ \mathrm{C} & 2.75549200 & -0.69978900 & -0.00005300 \\ \mathrm{H} & 4.36486300 & 2.32219300 & 0.00005200 \\ \mathrm{H} & 6.33313900 & 0.77355000 & -0.00002800 \\ \mathrm{H} & 5.98424100 & -1.66528600 & -0.00012200 \\ \mathrm{H} & 3.69087200 & -2.63790700 & -0.00013900 \\ \mathrm{C} & 1.61164300 & 1.29891300 & 0.00003700 \\ \mathrm{~N} & 1.38772200 & -1.05392300 & -0.00005400 \\ \mathrm{C} & 0.69898700 & 0.06777700 & -0.00000300 \\ \mathrm{C} & -0.70526700 & 0.08288600 & 0.00001000 \\ \mathrm{H} & -0.04135000 & -2.25410900 & -0.00008900 \\ \mathrm{O} & 1.24491600 & 2.45635500 & 0.00008600 \\ \mathrm{C} & -1.49759400 & -1.07648800 & -0.00002900 \\ \mathrm{~N} & -1.54843500 & 1.19244900 & 0.00006200 \\ \mathrm{H} & -1.21292900 & 2.14277700 & 0.00009700 \\ \mathrm{C} & -2.84936600 & 0.75906800 & 0.00005700 \\ \mathrm{C} & -2.85887000 & -0.66899900 & 0.00000000 \\ \mathrm{O} & -1.02852800 & -2.32112800 & -0.00008300 \\ \mathrm{C} & -4.04734500 & 1.48315700 & 0.00009700 \\ \mathrm{C} & -4.07817100 & -1.36870100 & -0.00001700 \\ \mathrm{C} & -5.23337700 & 0.76664100 & 0.00007900 \\ \mathrm{C} & -5.25477700 & -0.64607900 & 0.00002200 \\ \mathrm{H} & -4.04767400 & 2.56693500 & 0.00014000 \\ \mathrm{H} & -6.17355300 & 1.30657400 & 0.00010900 \\ \mathrm{H} & -6.20779600 & -1.16130800 & 0.00001000 \\ \mathrm{H} & -4.08120500 & -2.45205900 & -0.00006000\end{array}$




$\begin{array}{lrrr}\mathrm{C} & 2.95394900 & 0.69591400 & 0.00000000 \\ \mathrm{C} & 4.22316400 & 1.25414500 & 0.00001100 \\ \mathrm{C} & 5.32371800 & 0.39183600 & -0.00003400 \\ \mathrm{C} & 5.13005400 & -0.99203000 & -0.00008700 \\ \mathrm{C} & 3.84953200 & -1.55929300 & -0.00009800 \\ \mathrm{C} & 2.76002000 & -0.70117800 & -0.00005300 \\ \mathrm{H} & 4.35110200 & 2.33100600 & 0.00005200 \\ \mathrm{H} & 6.32938100 & 0.79444200 & -0.00002800 \\ \mathrm{H} & 5.99494300 & -1.64638600 & -0.00012100 \\ \mathrm{H} & 3.70800900 & -2.63323200 & -0.00013900 \\ \mathrm{C} & 1.60590200 & 1.29105200 & 0.00003700 \\ \mathrm{~N} & 1.39344000 & -1.06316200 & -0.00005500 \\ \mathrm{C} & 0.70009800 & 0.05526800 & -0.00000400 \\ \mathrm{C} & -0.70405800 & 0.07067200 & 0.00001000 \\ \mathrm{H} & -0.05156000 & -2.27053700 & -0.00009000 \\ \mathrm{O} & 1.23262100 & 2.44655500 & 0.00008600 \\ \mathrm{C} & -1.50175200 & -1.08452400 & -0.00002900 \\ \mathrm{~N} & -1.54122100 & 1.18451500 & 0.00006200 \\ \mathrm{H} & -1.19959800 & 2.13277100 & 0.00009600 \\ \mathrm{C} & -2.84391900 & 0.75824800 & 0.00005700 \\ \mathrm{C} & -2.86116600 & -0.66996300 & 0.00000000 \\ \mathrm{O} & -1.03812600 & -2.33189200 & -0.00008300 \\ \mathrm{C} & -4.03721900 & 1.48995400 & 0.00009700 \\ \mathrm{C} & -4.08517400 & -1.36174400 & -0.00001700 \\ \mathrm{C} & -5.22754400 & 0.78098300 & 0.00007900 \\ \mathrm{C} & -5.25722300 & -0.63165800 & 0.00002300 \\ \mathrm{H} & -4.03033700 & 2.57370800 & 0.00014000 \\ \mathrm{H} & -6.16447700 & 1.32651600 & 0.00011000 \\ \mathrm{H} & -6.21334400 & -1.14111400 & 0.00001100 \\ \mathrm{H} & -4.09551200 & -2.44506100 & -0.00006000\end{array}$


$\mathrm{S}_{0}$ Ind (IRC point 1 toward isomer 1), gas phase

$\begin{array}{lrrc}\mathrm{C} & 2.95484072 & 0.67027761 & -0.00000109 \\ \mathrm{C} & 4.25967372 & 1.14623961 & 0.00000591 \\ \mathrm{C} & 5.30403772 & 0.22149961 & -0.00004109 \\ \mathrm{C} & 5.02502572 & -1.14997839 & -0.00009209 \\ \mathrm{C} & 3.71602472 & -1.64035139 & -0.00010009 \\ \mathrm{C} & 2.67772372 & -0.71651039 & -0.00005309 \\ \mathrm{H} & 4.45029172 & 2.21369361 & 0.00004591 \\ \mathrm{H} & 6.33258572 & 0.56114561 & -0.00003709 \\ \mathrm{H} & 5.84844672 & -1.85584739 & -0.00012809 \\ \mathrm{H} & 3.51531972 & -2.70482939 & -0.00014009 \\ \mathrm{C} & 1.65703372 & 1.36698461 & 0.00003891 \\ \mathrm{~N} & 1.29813872 & -0.96328539 & -0.00005009 \\ \mathrm{C} & 0.66598072 & 0.22179061 & 0.00000291 \\ \mathrm{C} & -0.71411028 & 0.23992261 & 0.00001691 \\ \mathrm{H} & 0.33985972 & -1.84986439 & -0.00007609 \\ \mathrm{O} & 1.37211172 & 2.54972061 & 0.00008891 \\ \mathrm{C} & -1.42643328 & -1.00426939 & -0.00002609 \\ \mathrm{~N} & -1.62729328 & 1.28319561 & 0.00006691 \\ \mathrm{H} & -1.37230428 & 2.25724861 & 0.00010291 \\ \mathrm{C} & -2.89961928 & 0.75379461 & 0.00005691 \\ \mathrm{C} & -2.82285628 & -0.66979639 & -0.00000009 \\ \mathrm{O} & -0.82360728 & -2.14217939 & -0.00007709 \\ \mathrm{C} & -4.13665228 & 1.40342261 & 0.00009391 \\ \mathrm{C} & -3.99399928 & -1.44060939 & -0.00002109 \\ \mathrm{C} & -5.27921328 & 0.61531661 & 0.00007291 \\ \mathrm{C} & -5.21669728 & -0.79296239 & 0.00001591 \\ \mathrm{H} & -4.20317828 & 2.48512461 & 0.00013791 \\ \mathrm{H} & -6.24956728 & 1.09914161 & 0.00010091 \\ \mathrm{H} & -6.13585528 & -1.36607739 & 0.00000091 \\ \mathrm{H} & -3.92784628 & -2.52213439 & -0.00006509\end{array}$


$\mathrm{S}_{0}$ Ind (IRC point 20 toward isomer 1), gas phase

$\begin{array}{lrrr}\mathrm{C} & 2.94915500 & 0.66686600 & -0.00000100 \\ \mathrm{C} & 4.25812300 & 1.14344600 & 0.00000600 \\ \mathrm{C} & 5.30255500 & 0.22285800 & -0.00004100 \\ \mathrm{C} & 5.02460900 & -1.15115000 & -0.00009200 \\ \mathrm{C} & 3.72064800 & -1.64448000 & -0.00010000 \\ \mathrm{C} & 2.68217200 & -0.72192300 & -0.00005400 \\ \mathrm{H} & 4.44486000 & 2.21138800 & 0.00004600 \\ \mathrm{H} & 6.33104300 & 0.56254000 & -0.00003700 \\ \mathrm{H} & 5.84861800 & -1.85619400 & -0.00012800 \\ \mathrm{H} & 3.52450500 & -2.70994100 & -0.00014000 \\ \mathrm{C} & 1.65161700 & 1.36257000 & 0.00003900 \\ \mathrm{~N} & 1.32115400 & -0.95197100 & -0.00005000 \\ \mathrm{C} & 0.65332200 & 0.24023700 & 0.00000400 \\ \mathrm{C} & -0.70475300 & 0.25035300 & 0.00001700 \\ \mathrm{H} & 0.76664300 & -1.81465600 & -0.00007900 \\ \mathrm{O} & 1.37216000 & 2.55071200 & 0.00008900 \\ \mathrm{C} & -1.42320300 & -1.03235100 & -0.00002700 \\ \mathrm{~N} & -1.62219400 & 1.28235600 & 0.00006700 \\ \mathrm{H} & -1.37079000 & 2.25782500 & 0.00010300 \\ \mathrm{C} & -2.90274700 & 0.74954500 & 0.00005700 \\ \mathrm{C} & -2.83145800 & -0.66645500 & 0.00000000 \\ \mathrm{O} & -0.86267600 & -2.13983600 & -0.00007700 \\ \mathrm{C} & -4.13438500 & 1.40307100 & 0.00009400 \\ \mathrm{C} & -3.99242300 & -1.43874000 & -0.00002100 \\ \mathrm{C} & -5.28013900 & 0.61276700 & 0.00007300 \\ \mathrm{C} & -5.21977700 & -0.7915600 & 0.00001600 \\ \mathrm{H} & -4.20127800 & 2.48471500 & 0.00013800 \\ \mathrm{H} & -6.24972300 & 1.09835600 & 0.00010100 \\ \mathrm{H} & -6.13856900 & -1.36470400 & 0.00000100 \\ \mathrm{H} & -3.92121300 & -2.52021900 & -0.00006500\end{array}$


$\mathrm{S}_{0}$ Ind (IRC point 40 toward isomer 1), gas phase

$\begin{array}{lrrr}\mathrm{C} & 2.96073800 & 0.68024600 & -0.00000100 \\ \mathrm{C} & 4.26931700 & 1.15485000 & 0.00000600 \\ \mathrm{C} & 5.31380300 & 0.23383400 & -0.00004000 \\ \mathrm{C} & 5.04957100 & -1.14396900 & -0.00009300 \\ \mathrm{C} & 3.75012500 & -1.63990000 & -0.00010000 \\ \mathrm{C} & 2.71728000 & -0.70891900 & -0.00005300 \\ \mathrm{H} & 4.46020600 & 2.22193800 & 0.00004600 \\ \mathrm{H} & 6.34029200 & 0.57942900 & -0.00003600 \\ \mathrm{H} & 5.87985000 & -1.84150700 & -0.00012800 \\ \mathrm{H} & 3.54857100 & -2.70442100 & -0.00014000 \\ \mathrm{C} & 1.65132500 & 1.36374400 & 0.00003900 \\ \mathrm{~N} & 1.36320200 & -0.94809200 & -0.00005000 \\ \mathrm{C} & 0.66054200 & 0.23319200 & 0.00000400 \\ \mathrm{C} & -0.69925200 & 0.23051000 & 0.00001600 \\ \mathrm{H} & 0.87357700 & -1.83830900 & -0.00008100 \\ \mathrm{O} & 1.36852100 & 2.55173400 & 0.00008900 \\ \mathrm{C} & -1.45239200 & -1.05068100 & -0.00002800 \\ \mathrm{~N} & -1.61198800 & 1.26936900 & 0.00006600 \\ \mathrm{H} & -1.34994300 & 2.24236300 & 0.00010200 \\ \mathrm{C} & -2.90467200 & 0.75290900 & 0.00005700 \\ \mathrm{C} & -2.85186100 & -0.65769500 & 0.00000000 \\ \mathrm{O} & -0.95200900 & -2.17717200 & -0.00007700 \\ \mathrm{C} & -4.13474100 & 1.40810500 & 0.00009400 \\ \mathrm{C} & -4.00837200 & -1.43162900 & -0.00002000 \\ \mathrm{C} & -5.28480800 & 0.61952700 & 0.00007300 \\ \mathrm{C} & -5.23527600 & -0.78352500 & 0.00001600 \\ \mathrm{H} & -4.20236500 & 2.48964400 & 0.00013800 \\ \mathrm{H} & -6.25126300 & 1.11138400 & 0.00010100 \\ \mathrm{H} & -6.15685800 & -1.35250700 & 0.00000200 \\ \mathrm{H} & -3.93016500 & -2.51258900 & -0.00006400\end{array}$


$\mathrm{S}_{0}$ Ind (IRC point 60 toward isomer 1), gas phase

$\begin{array}{lrrr}\mathrm{C} & 2.96449700 & 0.68136500 & -0.00000100 \\ \mathrm{C} & 4.26757200 & 1.17576600 & 0.00000700 \\ \mathrm{C} & 5.32749200 & 0.27235700 & -0.00003900 \\ \mathrm{C} & 5.08324100 & -1.10913500 & -0.00009100 \\ \mathrm{C} & 3.79045400 & -1.62382700 & -0.00010000 \\ \mathrm{C} & 2.73986200 & -0.71108800 & -0.00005400 \\ \mathrm{H} & 4.44137300 & 2.24579600 & 0.00004800 \\ \mathrm{H} & 6.34842400 & 0.63411100 & -0.00003400 \\ \mathrm{H} & 5.92278000 & -1.79552500 & -0.00012700 \\ \mathrm{H} & 3.60716600 & -2.69171600 & -0.00014000 \\ \mathrm{C} & 1.64463100 & 1.34632400 & 0.00003800 \\ \mathrm{~N} & 1.38568500 & -0.96998400 & -0.00005100 \\ \mathrm{C} & 0.66449100 & 0.20259500 & 0.00000200 \\ \mathrm{C} & -0.69751800 & 0.19629400 & 0.00001500 \\ \mathrm{H} & 0.91395500 & -1.86848700 & -0.00008300 \\ \mathrm{O} & 1.34961000 & 2.53165500 & 0.00008800 \\ \mathrm{C} & -1.47495000 & -1.07571600 & -0.00002900 \\ \mathrm{~N} & -1.59864900 & 1.24698600 & 0.00006500 \\ \mathrm{H} & -1.32056400 & 2.21579900 & 0.00010100 \\ \mathrm{C} & -2.90074800 & 0.75225300 & 0.00005700 \\ \mathrm{C} & -2.87027000 & -0.65822300 & 0.00000100 \\ \mathrm{O} & -0.99798000 & -2.21044600 & -0.00007800 \\ \mathrm{C} & -4.12213700 & 1.42542200 & 0.00009500 \\ \mathrm{C} & -4.03890800 & -1.41437600 & -0.00001900 \\ \mathrm{C} & -5.28483100 & 0.65446400 & 0.00007400 \\ \mathrm{C} & -5.25698700 & -0.7489600 & 0.00001800 \\ \mathrm{H} & -4.17432200 & 2.50782500 & 0.00013800 \\ \mathrm{H} & -6.24342300 & 1.16152400 & 0.00010300 \\ \mathrm{H} & -6.18661200 & -1.30472800 & 0.00000400 \\ \mathrm{H} & -3.97637400 & -2.49640700 & -0.00006300\end{array}$


$\mathrm{S}_{0}$ Ind (IRC point 80 toward isomer 1), gas phase

$\begin{array}{lrrr}\mathrm{C} & 2.95783300 & 0.67887900 & -0.00000100 \\ \mathrm{C} & 4.25116000 & 1.19810300 & 0.00000800 \\ \mathrm{C} & 5.32784300 & 0.31535800 & -0.00003700 \\ \mathrm{C} & 5.10786400 & -1.07018000 & -0.00009000 \\ \mathrm{C} & 3.82445200 & -1.60926200 & -0.00010000 \\ \mathrm{C} & 2.75549000 & -0.71740700 & -0.00005400 \\ \mathrm{H} & 4.40337100 & 2.27141000 & 0.00004900 \\ \mathrm{H} & 6.34202900 & 0.69561500 & -0.00003200 \\ \mathrm{H} & 5.95950000 & -1.74151500 & -0.00012500 \\ \mathrm{H} & 3.66305300 & -2.68069900 & -0.00014100 \\ \mathrm{C} & 1.62918900 & 1.32249400 & 0.00003800 \\ \mathrm{~N} & 1.40332200 & -0.99740600 & -0.00005200 \\ \mathrm{C} & 0.66688500 & 0.16669100 & 0.00000100 \\ \mathrm{C} & -0.69450800 & 0.16250700 & 0.00001400 \\ \mathrm{H} & 0.94696000 & -1.90268200 & -0.00008400 \\ \mathrm{O} & 1.31578900 & 2.50386200 & 0.00008800 \\ \mathrm{C} & -1.48878600 & -1.09967800 & -0.00003000 \\ \mathrm{~N} & -1.58012700 & 1.22530400 & 0.00006400 \\ \mathrm{H} & -1.28501600 & 2.18938600 & 0.00010000 \\ \mathrm{C} & -2.88880600 & 0.75018700 & 0.00005700 \\ \mathrm{C} & -2.88046100 & -0.66099100 & 0.00000100 \\ \mathrm{O} & -1.02291000 & -2.23798000 & -0.00007900 \\ \mathrm{C} & -4.09765800 & 1.44539900 & 0.00009500 \\ \mathrm{C} & -4.06369600 & -1.39483900 & -0.00001800 \\ \mathrm{C} & -5.27411800 & 0.69606100 & 0.00007600 \\ \mathrm{C} & -5.27017600 & -0.70748900 & 0.00002000 \\ \mathrm{H} & -4.12919400 & 2.52859700 & 0.00013900 \\ \mathrm{H} & -6.22362000 & 1.21994900 & 0.00010500 \\ \mathrm{H} & -6.20920500 & -1.24722700 & 0.00000700 \\ \mathrm{H} & -4.02241000 & -2.47792400 & -0.00006200\end{array}$


$\mathrm{S}_{0}$ Ind (IRC point 100 toward isomer 1), gas phase

$\begin{array}{lrrr}\mathrm{C} & 2.94928600 & 0.67675500 & -0.00000100 \\ \mathrm{C} & 4.23248900 & 1.22004900 & 0.00000900 \\ \mathrm{C} & 5.32501400 & 0.35763400 & -0.00003600 \\ \mathrm{C} & 5.12923700 & -1.03153300 & -0.00008900 \\ \mathrm{C} & 3.85568200 & -1.59444900 & -0.00009900 \\ \mathrm{C} & 2.76958800 & -0.72316800 & -0.00005400 \\ \mathrm{H} & 4.36374700 & 2.29610600 & 0.00005100 \\ \mathrm{H} & 6.33226100 & 0.75589600 & -0.00002900 \\ \mathrm{H} & 5.99267100 & -1.68762900 & -0.00012300 \\ \mathrm{H} & 3.71541600 & -2.66887300 & -0.00014100 \\ \mathrm{C} & 1.61201900 & 1.29832800 & 0.00003700 \\ \mathrm{~N} & 1.42040400 & -1.02471000 & -0.00005400 \\ \mathrm{C} & 0.66922700 & 0.13040400 & -0.00000100 \\ \mathrm{C} & -0.69103600 & 0.12873300 & 0.00001200 \\ \mathrm{H} & 0.97959800 & -1.93669400 & -0.00008600 \\ \mathrm{O} & 1.27805000 & 2.47494900 & 0.00008700 \\ \mathrm{C} & -1.50137000 & -1.12314200 & -0.00003000 \\ \mathrm{~N} & -1.56005000 & 1.20378200 & 0.00006300 \\ \mathrm{H} & -1.24705200 & 2.16258400 & 0.00009800 \\ \mathrm{C} & -2.87472600 & 0.74840200 & 0.00005700 \\ \mathrm{C} & -2.88847700 & -0.66319900 & 0.00000000 \\ \mathrm{O} & -1.04668300 & -2.26509700 & -0.00008000 \\ \mathrm{C} & -4.07046500 & 1.46534000 & 0.00009600 \\ \mathrm{C} & -4.08537800 & -1.37487300 & -0.00001700 \\ \mathrm{C} & -5.25997200 & 0.73745400 & 0.00007800 \\ \mathrm{C} & -5.27976500 & -0.66583300 & 0.00002200 \\ \mathrm{H} & -4.08148900 & 2.54893000 & 0.00013900 \\ \mathrm{H} & -6.20025600 & 1.27770600 & 0.00010800 \\ \mathrm{H} & -6.22793500 & -1.18935700 & 0.00000900 \\ \mathrm{H} & -4.06495800 & -2.45857900 & -0.00006100\end{array}$


$\mathrm{S}_{0}$ Ind (IRC point 120 toward isomer 1), gas phase

$\begin{array}{lrrr}\mathrm{C} & 2.94056800 & 0.67483700 & 0.00000000 \\ \mathrm{C} & 4.21333200 & 1.24174600 & 0.00001000 \\ \mathrm{C} & 5.32111300 & 0.39965900 & -0.00003400 \\ \mathrm{C} & 5.14942800 & -0.99271200 & -0.00008800 \\ \mathrm{C} & 3.88609900 & -1.57899700 & -0.00009900 \\ \mathrm{C} & 2.78356900 & -0.72827700 & -0.00005500 \\ \mathrm{H} & 4.32390300 & 2.32011100 & 0.00005200 \\ \mathrm{H} & 6.32117700 & 0.81561700 & -0.00002700 \\ \mathrm{H} & 6.02430100 & -1.63347800 & -0.00012100 \\ \mathrm{H} & 3.76665600 & -2.65594900 & -0.00014100 \\ \mathrm{C} & 1.59501700 & 1.27422300 & 0.00003600 \\ \mathrm{~N} & 1.43789000 & -1.05136500 & -0.00005500 \\ \mathrm{C} & 0.67169800 & 0.09425300 & -0.00000200 \\ \mathrm{C} & -0.68760000 & 0.09463700 & 0.00001100 \\ \mathrm{H} & 1.01322700 & -1.97010700 & -0.00008700 \\ \mathrm{O} & 1.24084600 & 2.44584000 & 0.00008600 \\ \mathrm{C} & -1.51431700 & -1.14659800 & -0.00003100 \\ \mathrm{~N} & -1.53989600 & 1.18173700 & 0.00006200 \\ \mathrm{H} & -1.20882200 & 2.13487800 & 0.00009700 \\ \mathrm{C} & -2.86028700 & 0.74640800 & 0.00005600 \\ \mathrm{C} & -2.89626600 & -0.66518000 & 0.00000000 \\ \mathrm{O} & -1.07194000 & -2.29253700 & -0.00008100 \\ \mathrm{C} & -4.04252800 & 1.48481300 & 0.00009700 \\ \mathrm{C} & -4.10626500 & -1.35460300 & -0.00001600 \\ \mathrm{C} & -5.24463800 & 0.77849900 & 0.00007900 \\ \mathrm{C} & -5.28816300 & -0.62413600 & 0.00002300 \\ \mathrm{H} & -4.03312000 & 2.56840400 & 0.00014000 \\ \mathrm{H} & -6.17544900 & 1.33490800 & 0.00011000 \\ \mathrm{H} & -6.24519700 & -1.13128100 & 0.00001200 \\ \mathrm{H} & -4.10653400 & -2.43852500 & -0.00006000\end{array}$


$\mathrm{S}_{0}$ Ind (IRC point 140 toward isomer 1), gas phase

$\begin{array}{lrrr}\mathrm{C} & 2.93194100 & 0.67302800 & 0.00000000 \\ \mathrm{C} & 4.19390400 & 1.26324500 & 0.00001100 \\ \mathrm{C} & 5.31645400 & 0.44159800 & -0.00003200 \\ \mathrm{C} & 5.16878500 & -0.95356400 & -0.00008600 \\ \mathrm{C} & 3.91605600 & -1.56285500 & -0.00009900 \\ \mathrm{C} & 2.79764000 & -0.73278200 & -0.00005500 \\ \mathrm{H} & 4.28389700 & 2.34350700 & 0.00005300 \\ \mathrm{H} & 6.30907400 & 0.87501200 & -0.00002400 \\ \mathrm{H} & 6.05476400 & -1.57888600 & -0.00012000 \\ \mathrm{H} & 3.81727800 & -2.64191300 & -0.00014100 \\ \mathrm{C} & 1.57853400 & 1.25021100 & 0.00003500 \\ \mathrm{~N} & 1.45589200 & -1.07729200 & -0.00005600 \\ \mathrm{C} & 0.67432300 & 0.05834100 & -0.00000400 \\ \mathrm{C} & -0.68429300 & 0.06016200 & 0.00000900 \\ \mathrm{H} & 1.04789300 & -2.00277300 & -0.00008900 \\ \mathrm{O} & 1.20512500 & 2.41674800 & 0.00008500 \\ \mathrm{C} & -1.52788900 & -1.17015300 & -0.00003200 \\ \mathrm{~N} & -1.51996600 & 1.15902200 & 0.00006100 \\ \mathrm{H} & -1.17081500 & 2.10618100 & 0.00009500 \\ \mathrm{C} & -2.84580200 & 0.74406100 & 0.00005600 \\ \mathrm{C} & -2.90415100 & -0.66704500 & 0.00000000 \\ \mathrm{O} & -1.09909200 & -2.32040600 & -0.00008200 \\ \mathrm{C} & -4.01415200 & 1.50373000 & 0.00009700 \\ \mathrm{C} & -4.12677800 & -1.33406000 & -0.00001500 \\ \mathrm{C} & -5.22848300 & 0.81920500 & 0.00008100 \\ \mathrm{C} & -5.29578500 & -0.58238600 & 0.00002500 \\ \mathrm{H} & -3.98433000 & 2.58693700 & 0.00014000 \\ \mathrm{H} & -6.14953100 & 1.39162700 & 0.00011200 \\ \mathrm{H} & -6.26141800 & -1.07296900 & 0.00001400 \\ \mathrm{H} & -4.14765200 & -2.41780300 & -0.00005900\end{array}$


$\mathrm{S}_{0}$ Ind (IRC point 160 toward isomer 1), gas phase

$\begin{array}{lrrr}\mathrm{C} & 2.92364600 & 0.67118700 & 0.00000000 \\ \mathrm{C} & 4.17464100 & 1.28394200 & 0.00001200 \\ \mathrm{C} & 5.31118700 & 0.48244100 & -0.00003100 \\ \mathrm{C} & 5.18688700 & -0.91502200 & -0.00008500 \\ \mathrm{C} & 3.94487000 & -1.54647700 & -0.00009800 \\ \mathrm{C} & 2.81140200 & -0.73669600 & -0.00005500 \\ \mathrm{H} & 4.24439900 & 2.36576800 & 0.00005400 \\ \mathrm{H} & 6.29629700 & 0.93291800 & -0.00002200 \\ \mathrm{H} & 6.08354800 & -1.52503900 & -0.00011800 \\ \mathrm{H} & 3.86634800 & -2.62724200 & -0.00014000 \\ \mathrm{C} & 1.56313000 & 1.22688400 & 0.00003400 \\ \mathrm{~N} & 1.47386700 & -1.10162500 & -0.00005700 \\ \mathrm{C} & 0.67698800 & 0.02379400 & -0.00000500 \\ \mathrm{C} & -0.68127400 & 0.02629200 & 0.00000800 \\ \mathrm{H} & 1.08273900 & -2.03381800 & -0.00009000 \\ \mathrm{O} & 1.17237600 & 2.38853000 & 0.00008400 \\ \mathrm{C} & -1.54173500 & -1.19320900 & -0.00003300 \\ \mathrm{~N} & -1.50099700 & 1.13613600 & 0.00005900 \\ \mathrm{H} & -1.13445700 & 2.07721300 & 0.00009300 \\ \mathrm{C} & -2.83177700 & 0.74121000 & 0.00005600 \\ \mathrm{C} & -2.91200400 & -0.66891700 & 0.00000000 \\ \mathrm{O} & -1.12727300 & -2.34785700 & -0.00008300 \\ \mathrm{C} & -3.98612500 & 1.52148600 & 0.00009700 \\ \mathrm{C} & -4.14656500 & -1.31383900 & -0.00001400 \\ \mathrm{C} & -5.21204400 & 0.85853800 & 0.00008200 \\ \mathrm{C} & -5.30257600 & -0.54163300 & 0.00002700 \\ \mathrm{H} & -3.93611100 & 2.60404700 & 0.00014000 \\ \mathrm{H} & -6.12324200 & 1.44671500 & 0.00011400 \\ \mathrm{H} & -6.27646200 & -1.01576100 & 0.00001700 \\ \mathrm{H} & -4.18770200 & -2.39705700 & -0.00005700 \\ & & & \end{array}$




$\begin{array}{lrrr}\mathrm{C} & 2.92203200 & 0.67092500 & 0.00000000 \\ \mathrm{C} & 4.17080600 & 1.28820300 & 0.00001200 \\ \mathrm{C} & 5.31011200 & 0.49072200 & -0.00003000 \\ \mathrm{C} & 5.19051500 & -0.90718000 & -0.00008400 \\ \mathrm{C} & 3.95068000 & -1.54307500 & -0.00009800 \\ \mathrm{C} & 2.81426400 & -0.73736200 & -0.00005500 \\ \mathrm{H} & 4.23668500 & 2.37019500 & 0.00005500 \\ \mathrm{H} & 6.29365800 & 0.94437700 & -0.00002200 \\ \mathrm{H} & 6.08915400 & -1.51418900 & -0.00011700 \\ \mathrm{H} & 3.87612900 & -2.62412600 & -0.00014000 \\ \mathrm{C} & 1.56002800 & 1.22219400 & 0.00003400 \\ \mathrm{~N} & 1.47763000 & -1.10656100 & -0.00005700 \\ \mathrm{C} & 0.67759000 & 0.01673400 & -0.00000500 \\ \mathrm{C} & -0.68065100 & 0.01929000 & 0.00000700 \\ \mathrm{H} & 1.08973400 & -2.03992700 & -0.00009000 \\ \mathrm{O} & 1.16571700 & 2.38283600 & 0.00008400 \\ \mathrm{C} & -1.54465800 & -1.19794400 & -0.00003300 \\ \mathrm{~N} & -1.49710500 & 1.13144500 & 0.00005900 \\ \mathrm{H} & -1.12698100 & 2.07126700 & 0.00009300 \\ \mathrm{C} & -2.82890800 & 0.74069700 & 0.00005600 \\ \mathrm{C} & -2.91359600 & -0.66921500 & 0.00000000 \\ \mathrm{O} & -1.13344100 & -2.35358600 & -0.00008300 \\ \mathrm{C} & -3.98042700 & 1.52510400 & 0.00009700 \\ \mathrm{C} & -4.15049500 & -1.30971300 & -0.00001400 \\ \mathrm{C} & -5.20862200 & 0.86645700 & 0.00008300 \\ \mathrm{C} & -5.30385300 & -0.53340100 & 0.00002800 \\ \mathrm{H} & -3.92660000 & 2.60738100 & 0.00014000 \\ \mathrm{H} & -6.11782600 & 1.45752500 & 0.00011500 \\ \mathrm{H} & -6.27925100 & -1.00428400 & 0.00001800 \\ \mathrm{H} & -4.19556500 & -2.39276400 & -0.00005700\end{array}$


$\mathrm{S}_{1}$ Ind (IRC point 1 toward isomer 2), gas phase

$\begin{array}{lrrr}\mathrm{C} & 2.91847846 & 0.69261819 & -0.00001632 \\ \mathrm{C} & 4.22357146 & 1.13715619 & -0.00002332 \\ \mathrm{C} & 5.25475046 & 0.19149819 & -0.00004232 \\ \mathrm{C} & 4.98431946 & -1.20710981 & -0.00005532 \\ \mathrm{C} & 3.70016246 & -1.68818581 & -0.00004832 \\ \mathrm{C} & 2.63247346 & -0.73763481 & -0.00002932 \\ \mathrm{H} & 4.43638646 & 2.19990819 & -0.00001432 \\ \mathrm{H} & 6.28661146 & 0.52260519 & -0.00004832 \\ \mathrm{H} & 5.81808746 & -1.89925881 & -0.00006932 \\ \mathrm{H} & 3.48296046 & -2.74918681 & -0.00005832 \\ \mathrm{C} & 1.61705846 & 1.40515119 & 0.00000168 \\ \mathrm{~N} & 1.32878846 & -0.95945281 & -0.00002032 \\ \mathrm{C} & 0.67829646 & 0.32562219 & -0.00000132 \\ \mathrm{C} & -0.69329254 & 0.26396219 & 0.00000968 \\ \mathrm{H} & 0.38874046 & -1.82628481 & -0.00002132 \\ \mathrm{O} & 1.38346946 & 2.62907019 & 0.00001668 \\ \mathrm{C} & -1.39780554 & -1.00286981 & 0.00000168 \\ \mathrm{~N} & -1.61837454 & 1.28704219 & 0.00002768 \\ \mathrm{H} & -1.35832354 & 2.26153619 & 0.00003468 \\ \mathrm{C} & -2.88969154 & 0.74707919 & 0.00003168 \\ \mathrm{C} & -2.79608354 & -0.67486981 & 0.00001568 \\ \mathrm{O} & -0.80968854 & -2.14424381 & -0.00001532 \\ \mathrm{C} & -4.12970854 & 1.38299419 & 0.00004768 \\ \mathrm{C} & -3.95785154 & -1.45924681 & 0.00001668 \\ \mathrm{C} & -5.26688354 & 0.58107619 & 0.00004868 \\ \mathrm{C} & -5.18913654 & -0.82451581 & 0.00003368 \\ \mathrm{H} & -4.20746254 & 2.46385719 & 0.00005968 \\ \mathrm{H} & -6.24199154 & 1.05522619 & 0.00006168 \\ \mathrm{H} & -6.10169254 & -1.40816781 & 0.00003468 \\ \mathrm{H} & -3.87892154 & -2.53992781 & 0.00000568\end{array}$


$\mathrm{S}_{1}$ Ind (IRC point 20 toward isomer 2), gas phase

$\begin{array}{lrrr}\mathrm{C} & 2.93108300 & 0.70783600 & -0.00001700 \\ \mathrm{C} & 4.23735100 & 1.14707500 & -0.00002300 \\ \mathrm{C} & 5.26022000 & 0.19180600 & -0.00004300 \\ \mathrm{C} & 4.98796900 & -1.20350100 & -0.00005500 \\ \mathrm{C} & 3.69712500 & -1.67473900 & -0.00004800 \\ \mathrm{C} & 2.64727200 & -0.71425500 & -0.00002900 \\ \mathrm{H} & 4.46115600 & 2.20752300 & -0.00001400 \\ \mathrm{H} & 6.29371700 & 0.51873100 & -0.00004800 \\ \mathrm{H} & 5.81938100 & -1.89847600 & -0.00007000 \\ \mathrm{H} & 3.46545600 & -2.73294400 & -0.00005800 \\ \mathrm{C} & 1.62734800 & 1.40534600 & 0.00000200 \\ \mathrm{~N} & 1.34178500 & -0.95198800 & -0.00002000 \\ \mathrm{C} & 0.69401100 & 0.30001100 & -0.00000200 \\ \mathrm{C} & -0.69291600 & 0.24848500 & 0.00000900 \\ \mathrm{H} & 0.14526300 & -2.01202200 & -0.00002200 \\ \mathrm{O} & 1.37564500 & 2.62703300 & 0.00001700 \\ \mathrm{C} & -1.40798200 & -0.98483400 & 0.00000200 \\ \mathrm{~N} & -1.61628400 & 1.28449000 & 0.00002800 \\ \mathrm{H} & -1.34879100 & 2.25708500 & 0.00003500 \\ \mathrm{C} & -2.88565200 & 0.75320300 & 0.00003200 \\ \mathrm{C} & -2.79340900 & -0.67124400 & 0.00001600 \\ \mathrm{O} & -0.85862900 & -2.17836800 & -0.00001500 \\ \mathrm{C} & -4.13100400 & 1.38603000 & 0.00004800 \\ \mathrm{C} & -3.95734200 & -1.45977300 & 0.00001700 \\ \mathrm{C} & -5.26407200 & 0.58345700 & 0.00004900 \\ \mathrm{C} & -5.18509000 & -0.82566600 & 0.00003300 \\ \mathrm{H} & -4.21126600 & 2.46664600 & 0.00006000 \\ \mathrm{H} & -6.24019200 & 1.05543300 & 0.00006100 \\ \mathrm{H} & -6.09813300 & -1.40858500 & 0.00003500 \\ \mathrm{H} & -3.87888100 & -2.54030400 & 0.00000500 \\ & & & \end{array}$


$\mathrm{S}_{1}$ Ind (IRC point 40 toward isomer 2), gas phase

$\begin{array}{lrrr}\mathrm{C} & 2.94206700 & 0.72155200 & -0.00001700 \\ \mathrm{C} & 4.24979700 & 1.16487700 & -0.00002300 \\ \mathrm{C} & 5.27846200 & 0.21190400 & -0.00004200 \\ \mathrm{C} & 5.01294800 & -1.18136100 & -0.00005500 \\ \mathrm{C} & 3.71981700 & -1.65319500 & -0.00004800 \\ \mathrm{C} & 2.66878600 & -0.70084800 & -0.00002900 \\ \mathrm{H} & 4.47404000 & 2.22531800 & -0.00001400 \\ \mathrm{H} & 6.31031400 & 0.54444200 & -0.00004800 \\ \mathrm{H} & 5.84476200 & -1.87571500 & -0.00006900 \\ \mathrm{H} & 3.48872800 & -2.71158800 & -0.00005700 \\ \mathrm{C} & 1.62354000 & 1.39748900 & 0.00000200 \\ \mathrm{~N} & 1.35959500 & -0.96130500 & -0.00002000 \\ \mathrm{C} & 0.70305500 & 0.27198900 & -0.00000200 \\ \mathrm{C} & -0.68997800 & 0.21492500 & 0.00000900 \\ \mathrm{H} & 0.06449600 & -2.10859700 & -0.00002200 \\ \mathrm{O} & 1.34426700 & 2.61301500 & 0.00001700 \\ \mathrm{C} & -1.42890200 & -1.00030400 & 0.00000200 \\ \mathrm{~N} & -1.59783000 & 1.26714400 & 0.00002700 \\ \mathrm{H} & -1.30771400 & 2.23378300 & 0.00003500 \\ \mathrm{C} & -2.87788500 & 0.76062800 & 0.00003200 \\ \mathrm{C} & -2.80434300 & -0.66371300 & 0.00001600 \\ \mathrm{O} & -0.93002400 & -2.22327400 & -0.00001500 \\ \mathrm{C} & -4.12034100 & 1.40111300 & 0.00004800 \\ \mathrm{C} & -3.96962600 & -1.44775700 & 0.00001700 \\ \mathrm{C} & -5.25847700 & 0.60371500 & 0.00004900 \\ \mathrm{C} & -5.19224100 & -0.80625900 & 0.00003400 \\ \mathrm{H} & -4.19730200 & 2.48194900 & 0.00006000 \\ \mathrm{H} & -6.23117100 & 1.08272200 & 0.00006100 \\ \mathrm{H} & -6.11010200 & -1.38156400 & 0.00003500 \\ \mathrm{H} & -3.89379400 & -2.52840200 & 0.00000500\end{array}$


$S_{1}$ Ind (IRC point 60 toward isomer 2), gas phase

$\begin{array}{lrrr}\mathrm{C} & 2.93668700 & 0.72191100 & -0.00001700 \\ \mathrm{C} & 4.23689500 & 1.18737500 & -0.00002300 \\ \mathrm{C} & 5.28169400 & 0.25203400 & -0.00004200 \\ \mathrm{C} & 5.03826500 & -1.14463000 & -0.00005400 \\ \mathrm{C} & 3.75206900 & -1.63776800 & -0.00004800 \\ \mathrm{C} & 2.68425300 & -0.70484000 & -0.00002900 \\ \mathrm{H} & 4.44283800 & 2.25152900 & -0.00001400 \\ \mathrm{H} & 6.30792500 & 0.60159700 & -0.00004700 \\ \mathrm{H} & 5.88076700 & -1.82597800 & -0.00006900 \\ \mathrm{H} & 3.53974700 & -2.70010300 & -0.00005800 \\ \mathrm{C} & 1.60832700 & 1.37562400 & 0.00000100 \\ \mathrm{~N} & 1.37654500 & -0.98716400 & -0.00002100 \\ \mathrm{C} & 0.70620900 & 0.23566300 & -0.00000300 \\ \mathrm{C} & -0.68715500 & 0.18052900 & 0.00000800 \\ \mathrm{H} & 0.03553800 & -2.15678500 & -0.00002200 \\ \mathrm{O} & 1.30465300 & 2.58583500 & 0.00001700 \\ \mathrm{C} & -1.44177000 & -1.02280900 & 0.00000200 \\ \mathrm{~N} & -1.57811100 & 1.24606600 & 0.00002600 \\ \mathrm{H} & -1.26858100 & 2.20722600 & 0.00003400 \\ \mathrm{C} & -2.86477000 & 0.75979300 & 0.00003200 \\ \mathrm{C} & -2.81300500 & -0.66637100 & 0.00001600 \\ \mathrm{O} & -0.95664600 & -2.25375100 & -0.00001500 \\ \mathrm{C} & -4.09588500 & 1.42189000 & 0.00004800 \\ \mathrm{C} & -3.99298600 & -1.42914800 & 0.00001800 \\ \mathrm{C} & -5.24747800 & 0.64474400 & 0.00004900 \\ \mathrm{C} & -5.20434600 & -0.76625700 & 0.00003400 \\ \mathrm{H} & -4.15310800 & 2.50394700 & 0.00006000 \\ \mathrm{H} & -6.21199400 & 1.13998700 & 0.00006200 \\ \mathrm{H} & -6.13172400 & -1.32612300 & 0.00003600 \\ \mathrm{H} & -3.93750900 & -2.51104500 & 0.00000600\end{array}$


$\mathrm{S}_{1}$ Ind (IRC point 80 toward isomer 2), gas phase

$\begin{array}{rrrr}\mathrm{C} & 2.92840900 & 0.72093500 & -0.00001700 \\ \mathrm{C} & 4.21970800 & 1.20993700 & -0.00002300 \\ \mathrm{C} & 5.28068900 & 0.29345600 & -0.00004100 \\ \mathrm{C} & 5.06049800 & -1.10688000 & -0.00005400 \\ \mathrm{C} & 3.78272700 & -1.62302300 & -0.00004800 \\ \mathrm{C} & 2.69755500 & -0.71034600 & -0.00002900 \\ \mathrm{H} & 4.40578700 & 2.27773500 & -0.00001400 \\ \mathrm{H} & 6.30080900 & 0.66047400 & -0.00004600 \\ \mathrm{H} & 5.91444700 & -1.77383200 & -0.00006800 \\ \mathrm{H} & 3.59047600 & -2.68917800 & -0.00005700 \\ \mathrm{C} & 1.59156600 & 1.35254400 & 0.00000100 \\ \mathrm{~N} & 1.39272500 & -1.01423500 & -0.00002100 \\ \mathrm{C} & 0.70853700 & 0.19928400 & -0.00000300 \\ \mathrm{C} & -0.68401300 & 0.14682800 & 0.00000800 \\ \mathrm{H} & 0.01190700 & -2.19930000 & -0.00002200 \\ \mathrm{O} & 1.26460400 & 2.55735600 & 0.00001600 \\ \mathrm{C} & -1.45304700 & -1.04531800 & 0.00000200 \\ \mathrm{~N} & -1.55775700 & 1.22514700 & 0.00002600 \\ \mathrm{H} & -1.22899700 & 2.18053800 & 0.00003300 \\ \mathrm{C} & -2.84999900 & 0.75837000 & 0.00003100 \\ \mathrm{C} & -2.81995400 & -0.66947500 & 0.00001700 \\ \mathrm{O} & -0.97860200 & -2.28228600 & -0.00001500 \\ \mathrm{C} & -4.06881300 & 1.44257600 & 0.00004800 \\ \mathrm{C} & -4.01466400 & -1.41003900 & 0.00001800 \\ \mathrm{C} & -5.23362600 & 0.68648200 & 0.00004900 \\ \mathrm{C} & -5.21396500 & -0.72523600 & 0.00003500 \\ \mathrm{H} & -4.10539600 & 2.52551600 & 0.00006000 \\ \mathrm{H} & -6.18957500 & 1.19804000 & 0.00006200 \\ \mathrm{H} & -6.15084800 & -1.26907100 & 0.00003600 \\ \mathrm{H} & -3.98033000 & -2.49283100 & 0.00000700\end{array}$


$\mathrm{S}_{1}$ Ind (IRC point 100 toward isomer 2), gas phase

$\begin{array}{rrrr}\mathrm{C} & 2.91991900 & 0.72005800 & -0.00001700 \\ \mathrm{C} & 4.20187200 & 1.23242600 & -0.00002300 \\ \mathrm{C} & 5.27864100 & 0.33500700 & -0.00004100 \\ \mathrm{C} & 5.08174600 & -1.06862100 & -0.00005300 \\ \mathrm{C} & 3.81280600 & -1.60756900 & -0.00004800 \\ \mathrm{C} & 2.71082100 & -0.71530600 & -0.00003000 \\ \mathrm{H} & 4.36807100 & 2.30348800 & -0.00001300 \\ \mathrm{H} & 6.29236500 & 0.71934100 & -0.00004600 \\ \mathrm{H} & 5.94689300 & -1.72098900 & -0.00006700 \\ \mathrm{H} & 3.64056900 & -2.67714200 & -0.00005700 \\ \mathrm{C} & 1.57500500 & 1.32950100 & 0.00000100 \\ \mathrm{~N} & 1.40930200 & -1.04065600 & -0.00002200 \\ \mathrm{C} & 0.71094300 & 0.16314700 & -0.00000400 \\ \mathrm{C} & -0.68095300 & 0.11281800 & 0.00000700 \\ \mathrm{H} & -0.01307300 & -2.24244200 & -0.00002200 \\ \mathrm{O} & 1.22571000 & 2.52871600 & 0.00001600 \\ \mathrm{C} & -1.46464200 & -1.06792400 & 0.00000200 \\ \mathrm{~N} & -1.53747000 & 1.20365900 & 0.00002500 \\ \mathrm{H} & -1.18942600 & 2.15292800 & 0.00003200 \\ \mathrm{C} & -2.83498800 & 0.75657800 & 0.00003100 \\ \mathrm{C} & -2.82680400 & -0.67252700 & 0.00001600 \\ \mathrm{O} & -1.00194200 & -2.31115600 & -0.00001500 \\ \mathrm{C} & -4.04111100 & 1.46267900 & 0.00004800 \\ \mathrm{C} & -4.03572300 & -1.39071800 & 0.00001900 \\ \mathrm{C} & -5.21872300 & 0.72780700 & 0.00005000 \\ \mathrm{C} & -5.22256400 & -0.68425400 & 0.00003500 \\ \mathrm{H} & -4.05708300 & 2.54610900 & 0.00005900 \\ \mathrm{H} & -6.16584300 & 1.25551000 & 0.00006300 \\ \mathrm{H} & -6.16868200 & -1.21188400 & 0.00003700 \\ \mathrm{H} & -4.02242100 & -2.47398700 & 0.00000800\end{array}$




$\begin{array}{lrrr}\mathrm{C} & 2.91272100 & 0.71945500 & -0.00001700 \\ \mathrm{C} & 4.18637500 & 1.25160900 & -0.00002200 \\ \mathrm{C} & 5.27630600 & 0.37059700 & -0.00004000 \\ \mathrm{C} & 5.09926900 & -1.03551200 & -0.00005300 \\ \mathrm{C} & 3.83812600 & -1.59370200 & -0.00004800 \\ \mathrm{C} & 2.72225100 & -0.71896100 & -0.00003000 \\ \mathrm{H} & 4.33560800 & 2.32515700 & -0.00001300 \\ \mathrm{H} & 6.28435700 & 0.76957200 & -0.00004500 \\ \mathrm{H} & 5.97373200 & -1.67534200 & -0.00006700 \\ \mathrm{H} & 3.68290600 & -2.66588000 & -0.00005700 \\ \mathrm{C} & 1.56127200 & 1.31003000 & 0.00000100 \\ \mathrm{~N} & 1.42381200 & -1.06244900 & -0.00002200 \\ \mathrm{C} & 0.71309100 & 0.13268200 & -0.00000400 \\ \mathrm{C} & -0.67848700 & 0.08356800 & 0.00000700 \\ \mathrm{H} & -0.03576500 & -2.27997500 & -0.00002200 \\ \mathrm{O} & 1.19404600 & 2.50443600 & 0.00001600 \\ \mathrm{C} & -1.47489000 & -1.08729900 & 0.00000100 \\ \mathrm{~N} & -1.52047100 & 1.18480000 & 0.00002500 \\ \mathrm{H} & -1.15612700 & 2.12864000 & 0.00003100 \\ \mathrm{C} & -2.82224600 & 0.75461700 & 0.00003100 \\ \mathrm{C} & -2.83271900 & -0.67520000 & 0.00001600 \\ \mathrm{O} & -1.02322600 & -2.33610800 & -0.00001500 \\ \mathrm{C} & -4.01729100 & 1.47915300 & 0.00004800 \\ \mathrm{C} & -4.05332600 & -1.37425600 & 0.00001900 \\ \mathrm{C} & -5.20546800 & 0.76245600 & 0.00005000 \\ \mathrm{C} & -5.22928800 & -0.64959700 & 0.00003600 \\ \mathrm{H} & -4.01577500 & 2.56269000 & 0.00005900 \\ \mathrm{H} & -6.14487500 & 1.30375100 & 0.00006300 \\ \mathrm{H} & -6.18303000 & -1.16333900 & 0.00003800 \\ \mathrm{H} & -4.05781700 & -2.45760800 & 0.00000800\end{array}$


$\mathrm{S}_{1}$ Ind (IRC point 1 toward isomer 1), gas phase

$\begin{array}{lrrr}\mathrm{C} & 2.91847846 & 0.69261819 & -0.00001632 \\ \mathrm{C} & 4.22357146 & 1.13715619 & -0.00002332 \\ \mathrm{C} & 5.25475046 & 0.19149819 & -0.00004232 \\ \mathrm{C} & 4.98431946 & -1.20710981 & -0.00005532 \\ \mathrm{C} & 3.70016246 & -1.68818581 & -0.00004832 \\ \mathrm{C} & 2.63247346 & -0.73763481 & -0.00002932 \\ \mathrm{H} & 4.43638646 & 2.19990819 & -0.00001432 \\ \mathrm{H} & 6.28661146 & 0.52260519 & -0.00004832 \\ \mathrm{H} & 5.81808746 & -1.89925881 & -0.00006932 \\ \mathrm{H} & 3.48296046 & -2.74918681 & -0.00005832 \\ \mathrm{C} & 1.61705846 & 1.40515119 & 0.00000168 \\ \mathrm{~N} & 1.32878846 & -0.95945281 & -0.00002032 \\ \mathrm{C} & 0.67829646 & 0.32562219 & -0.00000132 \\ \mathrm{C} & -0.69329254 & 0.26396219 & 0.00000968 \\ \mathrm{H} & 0.38874046 & -1.82628481 & -0.00002132 \\ \mathrm{O} & 1.38346946 & 2.62907019 & 0.00001668 \\ \mathrm{C} & -1.39780554 & -1.00286981 & 0.00000168 \\ \mathrm{~N} & -1.61837454 & 1.28704219 & 0.00002768 \\ \mathrm{H} & -1.35832354 & 2.26153619 & 0.00003468 \\ \mathrm{C} & -2.88969154 & 0.74707919 & 0.00003168 \\ \mathrm{C} & -2.79608354 & -0.67486981 & 0.00001568 \\ \mathrm{O} & -0.80968854 & -2.14424381 & -0.00001532 \\ \mathrm{C} & -4.12970854 & 1.38299419 & 0.00004768 \\ \mathrm{C} & -3.95785154 & -1.45924681 & 0.00001668 \\ \mathrm{C} & -5.26688354 & 0.58107619 & 0.00004868 \\ \mathrm{C} & -5.18913654 & -0.82451581 & 0.00003368 \\ \mathrm{H} & -4.20746254 & 2.46385719 & 0.00005968 \\ \mathrm{H} & -6.24199154 & 1.05522619 & 0.00006168 \\ \mathrm{H} & -6.10169254 & -1.40816781 & 0.00003468 \\ \mathrm{H} & -3.87892154 & -2.53992781 & 0.00000568\end{array}$


$\mathrm{S}_{1}$ Ind (IRC point 20 toward isomer 1), gas phase

$\begin{array}{lrrr}\mathrm{C} & 2.92077100 & 0.69024300 & -0.00001700 \\ \mathrm{C} & 4.21904800 & 1.13572500 & -0.00002300 \\ \mathrm{C} & 5.25542300 & 0.18817900 & -0.00004300 \\ \mathrm{C} & 4.99044600 & -1.20920000 & -0.00005500 \\ \mathrm{C} & 3.70867900 & -1.69409000 & -0.00004900 \\ \mathrm{C} & 2.65359700 & -0.73678600 & -0.00002900 \\ \mathrm{H} & 4.42957800 & 2.19865100 & -0.00001400 \\ \mathrm{H} & 6.28602300 & 0.52225900 & -0.00004800 \\ \mathrm{H} & 5.82550900 & -1.89896900 & -0.00007000 \\ \mathrm{H} & 3.49136000 & -2.75481000 & -0.00005800 \\ \mathrm{C} & 1.60604600 & 1.40559400 & 0.00000200 \\ \mathrm{~N} & 1.35791900 & -0.93318000 & -0.00002000 \\ \mathrm{C} & 0.67009000 & 0.34279800 & -0.00000100 \\ \mathrm{C} & -0.69492300 & 0.26504800 & 0.00001000 \\ \mathrm{H} & 0.75617300 & -1.78483900 & -0.00002400 \\ \mathrm{O} & 1.38964300 & 2.62930100 & 0.00001700 \\ \mathrm{C} & -1.40110200 & -1.02628000 & 0.00000100 \\ \mathrm{~N} & -1.61279000 & 1.28166300 & 0.00002800 \\ \mathrm{H} & -1.35436000 & 2.25690400 & 0.00003500 \\ \mathrm{C} & -2.89763400 & 0.74337000 & 0.00003200 \\ \mathrm{C} & -2.80607100 & -0.67065300 & 0.00001600 \\ \mathrm{O} & -0.85774400 & -2.15468900 & -0.00001500 \\ \mathrm{C} & -4.12881800 & 1.38288500 & 0.00004800 \\ \mathrm{C} & -3.95794000 & -1.45628400 & 0.00001700 \\ \mathrm{C} & -5.27168200 & 0.57947400 & 0.00004900 \\ \mathrm{C} & -5.19381600 & -0.82107100 & 0.00003400 \\ \mathrm{H} & -4.20613600 & 2.46399200 & 0.00006000 \\ \mathrm{H} & -6.24574800 & 1.05549100 & 0.00006100 \\ \mathrm{H} & -6.10586200 & -1.40593000 & 0.00003500 \\ \mathrm{H} & -3.87280100 & -2.53665000 & 0.00000500\end{array}$


$\mathrm{S}_{1}$ Ind (IRC point 40 toward isomer 1), gas phase

$\begin{array}{lrrr}\mathrm{C} & 2.93766300 & 0.70189500 & -0.00001700 \\ \mathrm{C} & 4.23694900 & 1.14825200 & -0.00002300 \\ \mathrm{C} & 5.27804200 & 0.20251100 & -0.00004300 \\ \mathrm{C} & 5.02552800 & -1.19646700 & -0.00005500 \\ \mathrm{C} & 3.74412100 & -1.68260700 & -0.00004900 \\ \mathrm{C} & 2.68720900 & -0.72481100 & -0.00003000 \\ \mathrm{H} & 4.44907600 & 2.21087700 & -0.00001400 \\ \mathrm{H} & 6.30612600 & 0.54431300 & -0.00004800 \\ \mathrm{H} & 5.86433200 & -1.88162300 & -0.00007000 \\ \mathrm{H} & 3.52557300 & -2.74318400 & -0.00005800 \\ \mathrm{C} & 1.60979000 & 1.40722900 & 0.00000200 \\ \mathrm{~N} & 1.38951500 & -0.93010200 & -0.00002000 \\ \mathrm{C} & 0.67499500 & 0.33514100 & -0.00000100 \\ \mathrm{C} & -0.69637500 & 0.23647800 & 0.00000900 \\ \mathrm{H} & 0.83103200 & -1.79821000 & -0.00002500 \\ \mathrm{O} & 1.40048900 & 2.63178800 & 0.00001600 \\ \mathrm{C} & -1.43111200 & -1.05112000 & 0.00000100 \\ \mathrm{~N} & -1.60969700 & 1.25947800 & 0.00002700 \\ \mathrm{H} & -1.34072100 & 2.23217700 & 0.00003500 \\ \mathrm{C} & -2.90661400 & 0.74143400 & 0.00003200 \\ \mathrm{C} & -2.83203100 & -0.66993600 & 0.00001600 \\ \mathrm{O} & -0.92909800 & -2.19351200 & -0.00001500 \\ \mathrm{C} & -4.13376000 & 1.39056300 & 0.00004800 \\ \mathrm{C} & -3.98789500 & -1.44778900 & 0.00001700 \\ \mathrm{C} & -5.28495500 & 0.59590600 & 0.00004900 \\ \mathrm{C} & -5.22009900 & -0.80460600 & 0.00003400 \\ \mathrm{H} & -4.20463700 & 2.47215400 & 0.00006000 \\ \mathrm{H} & -6.25441800 & 1.08126600 & 0.00006200 \\ \mathrm{H} & -6.13658400 & -1.38255300 & 0.00003500 \\ \mathrm{H} & -3.90708700 & -2.52849700 & 0.00000500\end{array}$


$S_{1}$ Ind (IRC point 60 toward isomer 1), gas phase

$\begin{array}{lrrr}\mathrm{C} & 2.93729400 & 0.69958000 & -0.00001700 \\ \mathrm{C} & 4.22861400 & 1.16960100 & -0.00002300 \\ \mathrm{C} & 5.28720100 & 0.24359900 & -0.00004200 \\ \mathrm{C} & 5.05748200 & -1.15884700 & -0.00005500 \\ \mathrm{C} & 3.78369500 & -1.66730500 & -0.00004900 \\ \mathrm{C} & 2.70780000 & -0.73003300 & -0.00003000 \\ \mathrm{H} & 4.41990800 & 2.23617400 & -0.00001400 \\ \mathrm{H} & 6.30913400 & 0.60340600 & -0.00004700 \\ \mathrm{H} & 5.90710200 & -1.83055600 & -0.00006900 \\ \mathrm{H} & 3.58636800 & -2.73207400 & -0.00005800 \\ \mathrm{C} & 1.60029500 & 1.38559100 & 0.00000200 \\ \mathrm{~N} & 1.41022300 & -0.95334200 & -0.00002100 \\ \mathrm{C} & 0.67707500 & 0.30257500 & -0.00000200 \\ \mathrm{C} & -0.69632300 & 0.20302700 & 0.00000900 \\ \mathrm{H} & 0.87088400 & -1.82965100 & -0.00002600 \\ \mathrm{O} & 1.37961000 & 2.60835600 & 0.00001600 \\ \mathrm{C} & -1.44920400 & -1.07619500 & 0.00000100 \\ \mathrm{~N} & -1.59699800 & 1.23638800 & 0.00002700 \\ \mathrm{H} & -1.31349000 & 2.20534300 & 0.00003400 \\ \mathrm{C} & -2.90193300 & 0.73758200 & 0.00003200 \\ \mathrm{C} & -2.84871100 & -0.67478200 & 0.00001700 \\ \mathrm{O} & -0.95790800 & -2.22172300 & -0.00001500 \\ \mathrm{C} & -4.11720600 & 1.40890200 & 0.00004800 \\ \mathrm{C} & -4.02062100 & -1.42995600 & 0.00001800 \\ \mathrm{C} & -5.28348900 & 0.63639700 & 0.00004900 \\ \mathrm{C} & -5.24217300 & -0.76470200 & 0.00003400 \\ \mathrm{H} & -4.16721900 & 2.49169400 & 0.00006000 \\ \mathrm{H} & -6.24398700 & 1.13924700 & 0.00006200 \\ \mathrm{H} & -6.16818400 & -1.32734500 & 0.00003600 \\ \mathrm{H} & -3.96177500 & -2.51214200 & 0.00000600\end{array}$


$\mathrm{S}_{1}$ Ind (IRC point 80 toward isomer 1), gas phase

$\begin{array}{lrrr}\mathrm{C} & 2.93045500 & 0.69724800 & -0.00001700 \\ \mathrm{C} & 4.21252100 & 1.19110500 & -0.00002300 \\ \mathrm{C} & 5.28781900 & 0.28478200 & -0.00004200 \\ \mathrm{C} & 5.08227800 & -1.12103000 & -0.00005400 \\ \mathrm{C} & 3.81729100 & -1.65270500 & -0.00004900 \\ \mathrm{C} & 2.72386500 & -0.73608300 & -0.00003000 \\ \mathrm{H} & 4.38317800 & 2.26115800 & -0.00001300 \\ \mathrm{H} & 6.30320100 & 0.66266800 & -0.00004600 \\ \mathrm{H} & 5.94337100 & -1.77794000 & -0.00006900 \\ \mathrm{H} & 3.64083700 & -2.72115400 & -0.00005900 \\ \mathrm{C} & 1.58404400 & 1.36115000 & 0.00000100 \\ \mathrm{~N} & 1.42799600 & -0.97941200 & -0.00002100 \\ \mathrm{C} & 0.67856700 & 0.26577100 & -0.00000200 \\ \mathrm{C} & -0.69448000 & 0.16940200 & 0.00000800 \\ \mathrm{H} & 0.90560200 & -1.86336900 & -0.00002600 \\ \mathrm{O} & 1.34140700 & 2.58032800 & 0.00001600 \\ \mathrm{C} & -1.46241800 & -1.09966000 & 0.00000100 \\ \mathrm{~N} & -1.57838500 & 1.21521400 & 0.00002600 \\ \mathrm{H} & -1.27677400 & 2.17922500 & 0.00003400 \\ \mathrm{C} & -2.89008800 & 0.73598500 & 0.00003200 \\ \mathrm{C} & -2.85840100 & -0.67762900 & 0.00001700 \\ \mathrm{O} & -0.97963900 & -2.24805400 & -0.00001500 \\ \mathrm{C} & -4.09243200 & 1.42926300 & 0.00004800 \\ \mathrm{C} & -4.04461800 & -1.41050100 & 0.00001800 \\ \mathrm{C} & -5.27249700 & 0.67822000 & 0.00005000 \\ \mathrm{C} & -5.25458000 & -0.72313600 & 0.00003500 \\ \mathrm{H} & -4.12171700 & 2.51281800 & 0.00006000 \\ \mathrm{H} & -6.22410400 & 1.19766400 & 0.00006300 \\ \mathrm{H} & -6.19005200 & -1.26995400 & 0.00003700 \\ \mathrm{H} & -4.00698600 & -2.49366700 & 0.00000700\end{array}$


$\mathrm{S}_{1}$ Ind (IRC point 100 toward isomer 1), gas phase

$\begin{array}{rrrr}\mathrm{C} & 2.92325900 & 0.69536300 & -0.00001700 \\ \mathrm{C} & 4.19589600 & 1.21233900 & -0.00002300 \\ \mathrm{C} & 5.28720700 & 0.32552100 & -0.00004100 \\ \mathrm{C} & 5.10564300 & -1.08319000 & -0.00005400 \\ \mathrm{C} & 3.84970100 & -1.63742200 & -0.00004900 \\ \mathrm{C} & 2.73971800 & -0.74125900 & -0.00003000 \\ \mathrm{H} & 4.34648600 & 2.28538400 & -0.00001300 \\ \mathrm{H} & 6.29582500 & 0.72110000 & -0.00004600 \\ \mathrm{H} & 5.97778800 & -1.72532300 & -0.00006800 \\ \mathrm{H} & 3.69355800 & -2.70905300 & -0.00005900 \\ \mathrm{C} & 1.56758900 & 1.33672200 & 0.00000100 \\ \mathrm{~N} & 1.44600300 & -1.00482400 & -0.00002200 \\ \mathrm{C} & 0.68024300 & 0.22891000 & -0.00000300 \\ \mathrm{C} & -0.69250500 & 0.13550000 & 0.00000800 \\ \mathrm{H} & 0.94134700 & -1.89672900 & -0.00002700 \\ \mathrm{O} & 1.30233100 & 2.55182600 & 0.00001600 \\ \mathrm{C} & -1.47573200 & -1.12303800 & 0.00000100 \\ \mathrm{~N} & -1.55934300 & 1.19367600 & 0.00002600 \\ \mathrm{H} & -1.23917600 & 2.15231500 & 0.00003300 \\ \mathrm{C} & -2.87754200 & 0.73435600 & 0.00003100 \\ \mathrm{C} & -2.86755600 & -0.68011300 & 0.00001700 \\ \mathrm{O} & -1.00257300 & -2.27470500 & -0.00001500 \\ \mathrm{C} & -4.06663700 & 1.44920900 & 0.00004800 \\ \mathrm{C} & -4.06736100 & -1.39073100 & 0.00001900 \\ \mathrm{C} & -5.25994700 & 0.71962000 & 0.00005000 \\ \mathrm{C} & -5.26537200 & -0.68161300 & 0.00003600 \\ \mathrm{H} & -4.07540900 & 2.53312600 & 0.00006000 \\ \mathrm{H} & -6.20244800 & 1.25537500 & 0.00006300 \\ \mathrm{H} & -6.21001200 & -1.21248400 & 0.00003800 \\ \mathrm{H} & -4.05060800 & -2.47445400 & 0.00000800\end{array}$




$\begin{array}{lrrr}\mathrm{C} & 2.92038000 & 0.69468000 & -0.00001700 \\ \mathrm{C} & 4.18916700 & 1.22080500 & -0.00002200 \\ \mathrm{C} & 5.28674700 & 0.34181700 & -0.00004100 \\ \mathrm{C} & 5.11475000 & -1.06793200 & -0.00005400 \\ \mathrm{C} & 3.86251100 & -1.63107700 & -0.00004900 \\ \mathrm{C} & 2.74609100 & -0.74310900 & -0.00003000 \\ \mathrm{H} & 4.33178300 & 2.29493200 & -0.00001300 \\ \mathrm{H} & 6.29258500 & 0.74440900 & -0.00004500 \\ \mathrm{H} & 5.99122100 & -1.70413400 & -0.00006800 \\ \mathrm{H} & 3.71442900 & -2.70386200 & -0.00005900 \\ \mathrm{C} & 1.56110600 & 1.32695500 & 0.00000100 \\ \mathrm{~N} & 1.45333600 & -1.01479100 & -0.00002200 \\ \mathrm{C} & 0.68097300 & 0.21420500 & -0.00000300 \\ \mathrm{C} & -0.69172400 & 0.12185200 & 0.00000700 \\ \mathrm{H} & 0.95602600 & -1.90994700 & -0.00002700 \\ \mathrm{O} & 1.28693700 & 2.54034800 & 0.00001600 \\ \mathrm{C} & -1.48120300 & -1.13239600 & 0.00000100 \\ \mathrm{~N} & -1.55174300 & 1.18490300 & 0.00002500 \\ \mathrm{H} & -1.22411700 & 2.14129700 & 0.00003300 \\ \mathrm{C} & -2.87247900 & 0.73363600 & 0.00003100 \\ \mathrm{C} & -2.87121600 & -0.68104900 & 0.00001700 \\ \mathrm{O} & -1.01227000 & -2.28549300 & -0.00001500 \\ \mathrm{C} & -4.05619900 & 1.45701800 & 0.00004800 \\ \mathrm{C} & -4.07628300 & -1.38276800 & 0.00001900 \\ \mathrm{C} & -5.25467600 & 0.73602700 & 0.00005000 \\ \mathrm{C} & -5.26942400 & -0.66505000 & 0.00003600 \\ \mathrm{H} & -4.05681900 & 2.54096900 & 0.00005900 \\ \mathrm{H} & -6.19346800 & 1.27824400 & 0.00006300 \\ \mathrm{H} & -6.21764600 & -1.18951400 & 0.00003800 \\ \mathrm{H} & -4.06780500 & -2.46660000 & 0.00000800\end{array}$


$\mathrm{S}_{0}$ NHxInd (IRC point 1 toward isomer 2), gas phase

$\begin{array}{lrrr}\mathrm{C} & -2.07835282 & -2.36103533 & 0.22240536 \\ \mathrm{C} & -2.77557882 & -3.55141933 & 0.38488036 \\ \mathrm{C} & -4.11176782 & -3.59325533 & -0.01250364 \\ \mathrm{C} & -4.71665282 & -2.45368933 & -0.55722264 \\ \mathrm{C} & -4.02186182 & -1.25257533 & -0.72221164 \\ \mathrm{C} & -2.68923282 & -1.21740033 & -0.32509064 \\ \mathrm{H} & -2.28301982 & -4.41862233 & 0.81039836 \\ \mathrm{H} & -4.68541382 & -4.50529833 & 0.09876836 \\ \mathrm{H} & -5.75676982 & -2.50307233 & -0.86062164 \\ \mathrm{H} & -4.50281582 & -0.37846633 & -1.14421264 \\ \mathrm{C} & -0.68970082 & -2.00145533 & 0.54265836 \\ \mathrm{~N} & -1.79706082 & -0.14681433 & -0.38822964 \\ \mathrm{C} & -0.60128182 & -0.53071033 & 0.09959536 \\ \mathrm{C} & 0.42523618 & 0.41729267 & 0.09481736 \\ \mathrm{H} & -1.66094882 & 1.11156767 & -0.75935564 \\ \mathrm{O} & 0.18240418 & -2.68625033 & 1.03910236 \\ \mathrm{C} & 0.13048918 & 1.73065467 & -0.42774964 \\ \mathrm{~N} & 1.76159718 & 0.40432967 & 0.51191936 \\ \mathrm{C} & 2.30564818 & 1.64566867 & 0.24671736 \\ \mathrm{C} & 1.32722118 & 2.50198667 & -0.32525264 \\ \mathrm{O} & -1.02404382 & 2.07723167 & -0.88445064 \\ \mathrm{C} & 3.60743118 & 2.11289467 & 0.46984636 \\ \mathrm{C} & 1.63858718 & 3.82596967 & -0.66482164 \\ \mathrm{C} & 3.89234618 & 3.42672667 & 0.12517936 \\ \mathrm{C} & 2.92361018 & 4.28317067 & -0.43666564 \\ \mathrm{H} & 4.37475418 & 1.47780767 & 0.89361136 \\ \mathrm{H} & 4.89498718 & 3.80463267 & 0.29206236 \\ \mathrm{H} & 3.19416018 & 5.30095967 & -0.69001964 \\ \mathrm{H} & 0.87775218 & 4.46492367 & -1.09716064 \\ \mathrm{C} & 2.53372318 & -0.72876133 & 1.02417336 \\ \mathrm{C} & 3.23905818 & -1.52743333 & -0.07385964 \\ \mathrm{H} & 1.85620918 & -1.37820333 & 1.57161936 \\ \mathrm{H} & 3.25853118 & -0.32789533 & 1.73707736 \\ \mathrm{H} & 2.51052018 & -1.97293733 & -0.75392064 \\ \mathrm{H} & 3.91909118 & -0.89983233 & -0.65514664 \\ \mathrm{H} & 3.81766118 & -2.33985233 & 0.37390936\end{array}$


$\mathrm{S}_{0}$ NHxInd (IRC point 20 toward isomer 2), gas phase

$\begin{array}{lrrr}\mathrm{C} & -2.08109600 & -2.38471900 & 0.22979400 \\ \mathrm{C} & -2.78042500 & -3.57164200 & 0.38993800 \\ \mathrm{C} & -4.11873200 & -3.60558200 & -0.01082200 \\ \mathrm{C} & -4.72102900 & -2.46603700 & -0.55462200 \\ \mathrm{C} & -4.01908800 & -1.26781200 & -0.71608200 \\ \mathrm{C} & -2.68969600 & -1.24364800 & -0.31601200 \\ \mathrm{H} & -2.29577800 & -4.44402900 & 0.81427700 \\ \mathrm{H} & -4.69456400 & -4.51661800 & 0.09897500 \\ \mathrm{H} & -5.76093000 & -2.51348900 & -0.85895900 \\ \mathrm{H} & -4.49037500 & -0.38807400 & -1.13704200 \\ \mathrm{C} & -0.69101000 & -2.01092200 & 0.54501200 \\ \mathrm{~N} & -1.80889200 & -0.16224200 & -0.38685600 \\ \mathrm{C} & -0.62402100 & -0.52732000 & 0.09077700 \\ \mathrm{C} & 0.41462600 & 0.43374400 & 0.08536100 \\ \mathrm{H} & -1.62762400 & 1.35679000 & -0.82827500 \\ \mathrm{O} & 0.18583400 & -2.68567500 & 1.03919900 \\ \mathrm{C} & 0.14839600 & 1.73574800 & -0.42459700 \\ \mathrm{~N} & 1.75705800 & 0.40706700 & 0.50855600 \\ \mathrm{C} & 2.31061500 & 1.64206600 & 0.24991800 \\ \mathrm{C} & 1.33425900 & 2.50106000 & -0.32275400 \\ \mathrm{O} & -1.00519900 & 2.15229000 & -0.90278600 \\ \mathrm{C} & 3.61340900 & 2.11132400 & 0.47272900 \\ \mathrm{C} & 1.64361600 & 3.82721600 & -0.66360400 \\ \mathrm{C} & 3.89696000 & 3.42409400 & 0.12755700 \\ \mathrm{C} & 2.92686400 & 4.28187700 & -0.43534800 \\ \mathrm{H} & 4.38191900 & 1.47745600 & 0.89657900 \\ \mathrm{H} & 4.89939500 & 3.80265100 & 0.29403400 \\ \mathrm{H} & 3.19932000 & 5.29941400 & -0.68823700 \\ \mathrm{H} & 0.88268100 & 4.46578900 & -1.09577100 \\ \mathrm{C} & 2.52688500 & -0.72783900 & 1.02195100 \\ \mathrm{C} & 3.23594500 & -1.52706000 & -0.07358700 \\ \mathrm{H} & 1.85036900 & -1.37661400 & 1.57091000 \\ \mathrm{H} & 3.25080300 & -0.32557500 & 1.73482000 \\ \mathrm{H} & 2.51054800 & -1.97388700 & -0.75624000 \\ \mathrm{H} & 3.91642000 & -0.89841700 & -0.65304700 \\ \mathrm{H} & 3.81461300 & -2.33825000 & 0.37640500\end{array}$


$\mathrm{S}_{0}$ NHxInd (IRC point 40 toward isomer 2), gas phase

$\begin{array}{lrrr}\mathrm{C} & -2.07831700 & -2.38543800 & 0.23007900 \\ \mathrm{C} & -2.75750500 & -3.58296300 & 0.40074900 \\ \mathrm{C} & -4.09732000 & -3.64018400 & 0.00838800 \\ \mathrm{C} & -4.71761600 & -2.51133700 & -0.53703000 \\ \mathrm{C} & -4.03384500 & -1.30269800 & -0.70835300 \\ \mathrm{C} & -2.70212800 & -1.25350600 & -0.31719100 \\ \mathrm{H} & -2.25516100 & -4.44450800 & 0.82657000 \\ \mathrm{H} & -4.65948000 & -4.55872000 & 0.12552500 \\ \mathrm{H} & -5.75835700 & -2.57361900 & -0.83580000 \\ \mathrm{H} & -4.52227500 & -0.43308800 & -1.13116400 \\ \mathrm{C} & -0.69257400 & -1.99515900 & 0.53578000 \\ \mathrm{~N} & -1.83240200 & -0.15673700 & -0.39818900 \\ \mathrm{C} & -0.64355200 & -0.50895500 & 0.07569800 \\ \mathrm{C} & 0.39688900 & 0.45458700 & 0.07080700 \\ \mathrm{H} & -1.63437800 & 1.41689600 & -0.85101000 \\ \mathrm{O} & 0.19085800 & -2.66326000 & 1.02666200 \\ \mathrm{C} & 0.14916400 & 1.76098000 & -0.43395200 \\ \mathrm{~N} & 1.73863000 & 0.41245900 & 0.49930000 \\ \mathrm{C} & 2.30738100 & 1.64054200 & 0.24897500 \\ \mathrm{C} & 1.34382600 & 2.51336200 & -0.32446700 \\ \mathrm{O} & -0.99911600 & 2.19792200 & -0.91647700 \\ \mathrm{C} & 3.61603200 & 2.09017800 & 0.48144200 \\ \mathrm{C} & 1.67561400 & 3.83730000 & -0.65678700 \\ \mathrm{C} & 3.92027500 & 3.39978400 & 0.14465200 \\ \mathrm{C} & 2.96405300 & 4.27272400 & -0.41938300 \\ \mathrm{H} & 4.37288600 & 1.44305400 & 0.90591400 \\ \mathrm{H} & 4.92675400 & 3.76417000 & 0.31798900 \\ \mathrm{H} & 3.25084200 & 5.28779400 & -0.66634200 \\ \mathrm{H} & 0.92712900 & 4.48990300 & -1.08992200 \\ \mathrm{C} & 2.49962600 & -0.72547300 & 1.01865700 \\ \mathrm{C} & 3.20918700 & -1.52985900 & -0.07245200 \\ \mathrm{H} & 1.82010600 & -1.36955200 & 1.56779300 \\ \mathrm{H} & 3.22341100 & -0.32420800 & 1.73218300 \\ \mathrm{H} & 2.48474700 & -1.97550400 & -0.75678800 \\ \mathrm{H} & 3.89418300 & -0.90506200 & -0.65082000 \\ \mathrm{H} & 3.78331300 & -2.34228100 & 0.38121700\end{array}$




$\begin{array}{lrrr}\mathrm{C} & -2.07749600 & -2.38492300 & 0.22973300 \\ \mathrm{C} & -2.75436300 & -3.58350900 & 0.40171800 \\ \mathrm{C} & -4.09438300 & -3.64316500 & 0.01056800 \\ \mathrm{C} & -4.71673000 & -2.51550300 & -0.53495600 \\ \mathrm{C} & -4.03504700 & -1.30573000 & -0.70759100 \\ \mathrm{C} & -2.70305300 & -1.25397500 & -0.31757000 \\ \mathrm{H} & -2.25000700 & -4.44384600 & 0.82763600 \\ \mathrm{H} & -4.65506000 & -4.56249300 & 0.12868100 \\ \mathrm{H} & -5.75762900 & -2.57952900 & -0.83284500 \\ \mathrm{H} & -4.52547400 & -0.43728000 & -1.13050600 \\ \mathrm{C} & -0.69227400 & -1.99306300 & 0.53437300 \\ \mathrm{~N} & -1.83445800 & -0.15589200 & -0.39943700 \\ \mathrm{C} & -0.64520800 & -0.50694600 & 0.07415300 \\ \mathrm{C} & 0.39529500 & 0.45656500 & 0.06958600 \\ \mathrm{H} & -1.63496200 & 1.42098500 & -0.85291500 \\ \mathrm{O} & 0.19205500 & -2.66049900 & 1.02454800 \\ \mathrm{C} & 0.14898400 & 1.76320000 & -0.43492500 \\ \mathrm{~N} & 1.73675900 & 0.41304600 & 0.49876800 \\ \mathrm{C} & 2.30677900 & 1.64045700 & 0.24900400 \\ \mathrm{C} & 1.34442000 & 2.51453600 & -0.32468900 \\ \mathrm{O} & -0.99905500 & 2.20095100 & -0.91788600 \\ \mathrm{C} & 3.61598300 & 2.08802300 & 0.48242100 \\ \mathrm{C} & 1.67856500 & 3.83821800 & -0.65613200 \\ \mathrm{C} & 3.92234900 & 3.39725600 & 0.14646800 \\ \mathrm{C} & 2.96752400 & 4.27167900 & -0.41769500 \\ \mathrm{H} & 4.37160900 & 1.43950800 & 0.90694800 \\ \mathrm{H} & 4.92924200 & 3.76018300 & 0.32050000 \\ \mathrm{H} & 3.25580800 & 5.28648800 & -0.66402600 \\ \mathrm{H} & 0.93145400 & 4.49229900 & -1.08944200 \\ \mathrm{C} & 2.49691700 & -0.72512800 & 1.01880700 \\ \mathrm{C} & 3.20415500 & -1.53177500 & -0.07208000 \\ \mathrm{H} & 1.81751500 & -1.36763200 & 1.56978200 \\ \mathrm{H} & 3.22203600 & -0.32359900 & 1.73077800 \\ \mathrm{H} & 2.47835100 & -1.97755200 & -0.75484700 \\ \mathrm{H} & 3.88911400 & -0.90848000 & -0.65212200 \\ \mathrm{H} & 3.77792800 & -2.34424800 & 0.38194300\end{array}$


$\mathrm{S}_{0}$ NHxInd (IRC point 1 toward isomer 1), gas phase

$\begin{array}{lrrr}\mathrm{C} & -2.07835282 & -2.36103533 & 0.22240536 \\ \mathrm{C} & -2.77557882 & -3.55141933 & 0.38488036 \\ \mathrm{C} & -4.11176782 & -3.59325533 & -0.01250364 \\ \mathrm{C} & -4.71665282 & -2.45368933 & -0.55722264 \\ \mathrm{C} & -4.02186182 & -1.25257533 & -0.72221164 \\ \mathrm{C} & -2.68923282 & -1.21740033 & -0.32509064 \\ \mathrm{H} & -2.28301982 & -4.41862233 & 0.81039836 \\ \mathrm{H} & -4.68541382 & -4.50529833 & 0.09876836 \\ \mathrm{H} & -5.75676982 & -2.50307233 & -0.86062164 \\ \mathrm{H} & -4.50281582 & -0.37846633 & -1.14421264 \\ \mathrm{C} & -0.68970082 & -2.00145533 & 0.54265836 \\ \mathrm{~N} & -1.79706082 & -0.14681433 & -0.38822964 \\ \mathrm{C} & -0.60128182 & -0.53071033 & 0.09959536 \\ \mathrm{C} & 0.42523618 & 0.41729267 & 0.09481736 \\ \mathrm{H} & -1.66094882 & 1.11156767 & -0.75935564 \\ \mathrm{O} & 0.18240418 & -2.68625033 & 1.03910236 \\ \mathrm{C} & 0.13048918 & 1.73065467 & -0.42774964 \\ \mathrm{~N} & 1.76159718 & 0.40432967 & 0.51191936 \\ \mathrm{C} & 2.30564818 & 1.64566867 & 0.24671736 \\ \mathrm{C} & 1.32722118 & 2.50198667 & -0.32525264 \\ \mathrm{O} & -1.02404382 & 2.07723167 & -0.88445064 \\ \mathrm{C} & 3.60743118 & 2.11289467 & 0.46984636 \\ \mathrm{C} & 1.63858718 & 3.82596967 & -0.66482164 \\ \mathrm{C} & 3.89234618 & 3.42672667 & 0.12517936 \\ \mathrm{C} & 2.92361018 & 4.28317067 & -0.43666564 \\ \mathrm{H} & 4.37475418 & 1.47780767 & 0.89361136 \\ \mathrm{H} & 4.89498718 & 3.80463267 & 0.29206236 \\ \mathrm{H} & 3.19416018 & 5.30095967 & -0.69001964 \\ \mathrm{H} & 0.87775218 & 4.46492367 & -1.09716064 \\ \mathrm{C} & 2.53372318 & -0.72876133 & 1.02417336 \\ \mathrm{C} & 3.23905818 & -1.52743333 & -0.07385964 \\ \mathrm{H} & 1.85620918 & -1.37820333 & 1.57161936 \\ \mathrm{H} & 3.25853118 & -0.32789533 & 1.73707736 \\ \mathrm{H} & 2.51052018 & -1.97293733 & -0.75392064 \\ \mathrm{H} & 3.91909118 & -0.89983233 & -0.65514664 \\ \mathrm{H} & 3.81766118 & -2.33985233 & 0.37390936\end{array}$


$\mathrm{S}_{0}$ NHxInd (IRC point 20 toward isomer 1), gas phase

$\begin{array}{lrrr}\mathrm{C} & -2.07552100 & -2.35401800 & 0.22090900 \\ \mathrm{C} & -2.77585000 & -3.54772800 & 0.38361700 \\ \mathrm{C} & -4.10941200 & -3.59312200 & -0.01182200 \\ \mathrm{C} & -4.71718000 & -2.45251000 & -0.55776100 \\ \mathrm{C} & -4.02879700 & -1.25265900 & -0.72435600 \\ \mathrm{C} & -2.69646800 & -1.21657500 & -0.32783100 \\ \mathrm{H} & -2.27976700 & -4.41247100 & 0.80949400 \\ \mathrm{H} & -4.68274200 & -4.50527700 & 0.09969700 \\ \mathrm{H} & -5.75729400 & -2.50322700 & -0.86067100 \\ \mathrm{H} & -4.51401800 & -0.38103600 & -1.14686900 \\ \mathrm{C} & -0.68779600 & -1.99362100 & 0.54071000 \\ \mathrm{~N} & -1.80778600 & -0.16966700 & -0.38386600 \\ \mathrm{C} & -0.57777300 & -0.53644000 & 0.10875600 \\ \mathrm{C} & 0.42562200 & 0.40176300 & 0.10019100 \\ \mathrm{H} & -1.92803700 & 0.78750800 & -0.73408800 \\ \mathrm{O} & 0.18278900 & -2.68687700 & 1.03958500 \\ \mathrm{C} & 0.10821000 & 1.74846100 & -0.43982800 \\ \mathrm{~N} & 1.75564300 & 0.40053700 & 0.51127200 \\ \mathrm{C} & 2.30497200 & 1.65112200 & 0.24413500 \\ \mathrm{C} & 1.33622500 & 2.50655900 & -0.32404800 \\ \mathrm{O} & -0.99435300 & 2.10169600 & -0.88368900 \\ \mathrm{C} & 3.60519900 & 2.11130300 & 0.46960800 \\ \mathrm{C} & 1.63882400 & 3.82348200 & -0.66396200 \\ \mathrm{C} & 3.89119600 & 3.42938300 & 0.12399500 \\ \mathrm{C} & 2.92751400 & 4.28436700 & -0.43585300 \\ \mathrm{H} & 4.37265500 & 1.47648400 & 0.89321100 \\ \mathrm{H} & 4.89452900 & 3.80530300 & 0.29177100 \\ \mathrm{H} & 3.19743900 & 5.30215300 & -0.68944900 \\ \mathrm{H} & 0.87398800 & 4.45804200 & -1.09633800 \\ \mathrm{C} & 2.53344100 & -0.72932200 & 1.02457600 \\ \mathrm{C} & 3.23927000 & -1.52695100 & -0.07403500 \\ \mathrm{H} & 1.85583600 & -1.38120400 & 1.56937600 \\ \mathrm{H} & 3.25710600 & -0.32587200 & 1.73737200 \\ \mathrm{H} & 2.50948600 & -1.97311100 & -0.75218100 \\ \mathrm{H} & 3.91968300 & -0.90034300 & -0.65615300 \\ \mathrm{H} & 3.81744700 & -2.33918300 & 0.37447100\end{array}$


$\mathrm{S}_{0}$ NHxInd (IRC point 40 toward isomer 1), gas phase

$\begin{array}{lrrr}\mathrm{C} & -2.07641700 & -2.36970400 & 0.22448800 \\ \mathrm{C} & -2.77443100 & -3.56410400 & 0.38844100 \\ \mathrm{C} & -4.10871300 & -3.61426100 & -0.00511700 \\ \mathrm{C} & -4.73054200 & -2.48117600 & -0.55302100 \\ \mathrm{C} & -4.04929200 & -1.28000500 & -0.72206900 \\ \mathrm{C} & -2.71500700 & -1.24488300 & -0.32436700 \\ \mathrm{H} & -2.27793800 & -4.42855000 & 0.81423500 \\ \mathrm{H} & -4.67483500 & -4.53041700 & 0.11025200 \\ \mathrm{H} & -5.77102300 & -2.54223900 & -0.85261300 \\ \mathrm{H} & -4.53480700 & -0.40823400 & -1.14413700 \\ \mathrm{C} & -0.68762200 & -1.99457400 & 0.54109700 \\ \mathrm{~N} & -1.83409100 & -0.19296600 & -0.38172000 \\ \mathrm{C} & -0.58704000 & -0.53661300 & 0.10708400 \\ \mathrm{C} & 0.40996100 & 0.41132500 & 0.09264600 \\ \mathrm{H} & -1.99917300 & 0.74605800 & -0.73944700 \\ \mathrm{O} & 0.18424800 & -2.68659100 & 1.04115900 \\ \mathrm{C} & 0.11595800 & 1.78249200 & -0.44775100 \\ \mathrm{~N} & 1.74169100 & 0.40191900 & 0.50328900 \\ \mathrm{C} & 2.30904500 & 1.65097400 & 0.24295200 \\ \mathrm{C} & 1.35692700 & 2.51790500 & -0.32201100 \\ \mathrm{O} & -0.95891100 & 2.18379700 & -0.89908000 \\ \mathrm{C} & 3.61033400 & 2.10568100 & 0.47116500 \\ \mathrm{C} & 1.66097100 & 3.83294600 & -0.66034800 \\ \mathrm{C} & 3.90445400 & 3.42521400 & 0.12899100 \\ \mathrm{C} & 2.95080600 & 4.28978500 & -0.43048600 \\ \mathrm{H} & 4.37585200 & 1.46831100 & 0.89440000 \\ \mathrm{H} & 4.91029200 & 3.79250800 & 0.30093800 \\ \mathrm{H} & 3.22761800 & 5.30644400 & -0.68102300 \\ \mathrm{H} & 0.89444100 & 4.46590800 & -1.09198400 \\ \mathrm{C} & 2.51822100 & -0.72885900 & 1.02073200 \\ \mathrm{C} & 3.23714400 & -1.52118500 & -0.07360000 \\ \mathrm{H} & 1.83908700 & -1.38520300 & 1.55771300 \\ \mathrm{H} & 3.23422100 & -0.32345700 & 1.74027500 \\ \mathrm{H} & 2.51444200 & -1.97525600 & -0.75409900 \\ \mathrm{H} & 3.91495000 & -0.89002800 & -0.65382200 \\ \mathrm{H} & 3.82020100 & -2.32741900 & 0.37945500\end{array}$


$\mathrm{S}_{0}$ NHxInd (IRC point 60 toward isomer 1), gas phase

$\begin{array}{lrrr}\mathrm{C} & -2.07657500 & -2.36728500 & 0.22281200 \\ \mathrm{C} & -2.75225200 & -3.57334600 & 0.39615800 \\ \mathrm{C} & -4.08714800 & -3.64906600 & 0.01069100 \\ \mathrm{C} & -4.72854400 & -2.52748100 & -0.53815100 \\ \mathrm{C} & -4.06810500 & -1.31478700 & -0.71583700 \\ \mathrm{C} & -2.73141400 & -1.25222200 & -0.32629600 \\ \mathrm{H} & -2.23637100 & -4.42581100 & 0.82307800 \\ \mathrm{H} & -4.63766000 & -4.57387700 & 0.13262000 \\ \mathrm{H} & -5.76943800 & -2.60554400 & -0.83240300 \\ \mathrm{H} & -4.57340000 & -0.45485500 & -1.13903100 \\ \mathrm{C} & -0.69306500 & -1.97661300 & 0.53373500 \\ \mathrm{~N} & -1.86056800 & -0.18777600 & -0.38930500 \\ \mathrm{C} & -0.60577200 & -0.51836400 & 0.09601500 \\ \mathrm{C} & 0.39269900 & 0.42948700 & 0.08045500 \\ \mathrm{H} & -2.03832100 & 0.74656700 & -0.74944600 \\ \mathrm{O} & 0.18216600 & -2.66437800 & 1.03451300 \\ \mathrm{C} & 0.11465700 & 1.80847300 & -0.45657100 \\ \mathrm{~N} & 1.72389000 & 0.40688900 & 0.49241800 \\ \mathrm{C} & 2.30599600 & 1.64989900 & 0.23895400 \\ \mathrm{C} & 1.36698400 & 2.53140100 & -0.32480900 \\ \mathrm{O} & -0.95577100 & 2.21803200 & -0.90712800 \\ \mathrm{C} & 3.61355500 & 2.08253000 & 0.47579600 \\ \mathrm{C} & 1.69560400 & 3.84399100 & -0.65305000 \\ \mathrm{C} & 3.93072900 & 3.39886900 & 0.14368000 \\ \mathrm{C} & 2.99197700 & 4.28002200 & -0.41451300 \\ \mathrm{H} & 4.36631500 & 1.42998800 & 0.89853200 \\ \mathrm{H} & 4.94128900 & 3.74964600 & 0.32239100 \\ \mathrm{H} & 3.28424300 & 5.29410900 & -0.65790300 \\ \mathrm{H} & 0.94265400 & 4.49351100 & -1.08410100 \\ \mathrm{C} & 2.49301200 & -0.72594500 & 1.01564500 \\ \mathrm{C} & 3.22859500 & -1.51059900 & -0.07291700 \\ \mathrm{H} & 1.80778400 & -1.38595100 & 1.53886100 \\ \mathrm{H} & 3.19831500 & -0.32346100 & 1.74737200 \\ \mathrm{H} & 2.51623100 & -1.96374100 & -0.76491100 \\ \mathrm{H} & 3.91184000 & -0.87498700 & -0.64178000 \\ \mathrm{H} & 3.80843500 & -2.31703600 & 0.38390900\end{array}$




$\begin{array}{lrrr}\mathrm{C} & -2.07365100 & -2.36176700 & 0.22016400 \\ \mathrm{C} & -2.72763800 & -3.57799100 & 0.40140900 \\ \mathrm{C} & -4.06254000 & -3.67644200 & 0.02334500 \\ \mathrm{C} & -4.72222200 & -2.56550800 & -0.52536400 \\ \mathrm{C} & -4.08187100 & -1.34226200 & -0.71053600 \\ \mathrm{C} & -2.74380700 & -1.25576900 & -0.32863300 \\ \mathrm{H} & -2.19392700 & -4.41920700 & 0.82864000 \\ \mathrm{H} & -4.59865600 & -4.60893700 & 0.15056200 \\ \mathrm{H} & -5.76329800 & -2.65969900 & -0.81427300 \\ \mathrm{H} & -4.60494400 & -0.49310400 & -1.13393800 \\ \mathrm{C} & -0.69587400 & -1.95689200 & 0.52589100 \\ \mathrm{~N} & -1.88292500 & -0.18047400 & -0.39641400 \\ \mathrm{C} & -0.62262200 & -0.50058600 & 0.08643000 \\ \mathrm{C} & 0.37731800 & 0.44491800 & 0.07209900 \\ \mathrm{H} & -2.07053800 & 0.75028100 & -0.75802100 \\ \mathrm{O} & 0.18455500 & -2.63901800 & 1.02602200 \\ \mathrm{C} & 0.11173200 & 1.82858100 & -0.46257300 \\ \mathrm{~N} & 1.70716000 & 0.41008700 & 0.48624500 \\ \mathrm{C} & 2.30081000 & 1.64714100 & 0.23669900 \\ \mathrm{C} & 1.37285300 & 2.54066600 & -0.32639400 \\ \mathrm{O} & -0.95584400 & 2.24340100 & -0.91284500 \\ \mathrm{C} & 3.61364300 & 2.05950500 & 0.47935200 \\ \mathrm{C} & 1.72253800 & 3.85020200 & -0.64706600 \\ \mathrm{C} & 3.95058100 & 3.37208400 & 0.15460400 \\ \mathrm{C} & 3.02458000 & 4.26728800 & -0.40210700 \\ \mathrm{H} & 4.35519000 & 1.39385600 & 0.90104500 \\ \mathrm{H} & 4.96523200 & 3.70846100 & 0.33763000 \\ \mathrm{H} & 3.33062900 & 5.27858600 & -0.64013800 \\ \mathrm{H} & 0.98162700 & 4.51380200 & -1.07760600 \\ \mathrm{C} & 2.47261500 & -0.72328400 & 1.01375900 \\ \mathrm{C} & 3.21398700 & -1.50643700 & -0.07169300 \\ \mathrm{H} & 1.78575200 & -1.38345400 & 1.53303800 \\ \mathrm{H} & 3.17402000 & -0.32025900 & 1.74887300 \\ \mathrm{H} & 2.50506900 & -1.96074600 & -0.76644000 \\ \mathrm{H} & 3.89852400 & -0.86997900 & -0.63805400 \\ \mathrm{H} & 3.79330500 & -2.31194400 & 0.38746200\end{array}$


$\mathrm{S}_{1}$ NHxInd (IRC point 1 toward isomer 2), gas phase

$\begin{array}{lrrr}\mathrm{C} & -2.04121769 & -2.34095912 & 0.23879069 \\ \mathrm{C} & -2.75313769 & -3.51226812 & 0.39490669 \\ \mathrm{C} & -4.09255469 & -3.53629212 & -0.00645031 \\ \mathrm{C} & -4.72326369 & -2.38822112 & -0.56572731 \\ \mathrm{C} & -4.04382369 & -1.20830312 & -0.73471331 \\ \mathrm{C} & -2.67398269 & -1.16832412 & -0.32960831 \\ \mathrm{H} & -2.27550969 & -4.38670412 & 0.82127869 \\ \mathrm{H} & -4.67157969 & -4.44512412 & 0.10832869 \\ \mathrm{H} & -5.76350769 & -2.45704512 & -0.86160731 \\ \mathrm{H} & -4.51320969 & -0.32950112 & -1.15949131 \\ \mathrm{C} & -0.64115269 & -1.98796212 & 0.55676469 \\ \mathrm{~N} & -1.82261669 & -0.16083512 & -0.39045731 \\ \mathrm{C} & -0.55184869 & -0.60698412 & 0.13747069 \\ \mathrm{C} & 0.42061631 & 0.38448888 & 0.09683969 \\ \mathrm{H} & -1.68357269 & 1.07343788 & -0.75013331 \\ \mathrm{O} & 0.21553931 & -2.73870812 & 1.06423669 \\ \mathrm{C} & 0.10773331 & 1.71128188 & -0.43167031 \\ \mathrm{~N} & 1.75184431 & 0.38624088 & 0.49712769 \\ \mathrm{C} & 2.29156631 & 1.63410388 & 0.23379769 \\ \mathrm{C} & 1.30540431 & 2.48375388 & -0.33158831 \\ \mathrm{O} & -1.03715369 & 2.07507388 & -0.88590131 \\ \mathrm{C} & 3.58750231 & 2.10555388 & 0.45571269 \\ \mathrm{C} & 1.60593231 & 3.80960488 & -0.67084731 \\ \mathrm{C} & 3.86455731 & 3.42514288 & 0.11177769 \\ \mathrm{C} & 2.89065631 & 4.27503588 & -0.44570531 \\ \mathrm{H} & 4.35781031 & 1.47329988 & 0.87812669 \\ \mathrm{H} & 4.86524331 & 3.80856588 & 0.27774869 \\ \mathrm{H} & 3.15368631 & 5.29494088 & -0.69850031 \\ \mathrm{H} & 0.83926931 & 4.44395788 & -1.09963931 \\ \mathrm{C} & 2.52117831 & -0.74838812 & 1.01770469 \\ \mathrm{C} & 3.28412331 & -1.50541612 & -0.07105031 \\ \mathrm{H} & 1.82293731 & -1.42874312 & 1.50259369 \\ \mathrm{H} & 3.20514431 & -0.35524012 & 1.77473569 \\ \mathrm{H} & 2.58655631 & -1.95124912 & -0.78252231 \\ \mathrm{H} & 3.97494431 & -0.85628012 & -0.61525431 \\ \mathrm{H} & 3.85866031 & -2.31705512 & 0.38304269\end{array}$


$\mathrm{S}_{1}$ NHxInd (IRC point 20 toward isomer 2), gas phase

$\begin{array}{lrrr}\mathrm{C} & -2.04396400 & -2.36286700 & 0.24548000 \\ \mathrm{C} & -2.76006000 & -3.53144500 & 0.39924600 \\ \mathrm{C} & -4.10056000 & -3.54345700 & -0.00660700 \\ \mathrm{C} & -4.72491200 & -2.39753000 & -0.56328600 \\ \mathrm{C} & -4.03134500 & -1.21909600 & -0.72729200 \\ \mathrm{C} & -2.67185900 & -1.19734800 & -0.31940500 \\ \mathrm{H} & -2.29282200 & -4.41202800 & 0.82450600 \\ \mathrm{H} & -4.68337900 & -4.45048100 & 0.10615800 \\ \mathrm{H} & -5.76484800 & -2.45976900 & -0.86144100 \\ \mathrm{H} & -4.48865700 & -0.33305900 & -1.15093600 \\ \mathrm{C} & -0.64888500 & -1.99588400 & 0.55700600 \\ \mathrm{~N} & -1.82304600 & -0.17464700 & -0.38601700 \\ \mathrm{C} & -0.57993400 & -0.59846700 & 0.12554300 \\ \mathrm{C} & 0.40964200 & 0.39797500 & 0.08852400 \\ \mathrm{H} & -1.64487900 & 1.34069600 & -0.82493500 \\ \mathrm{O} & 0.22212000 & -2.73043000 & 1.06262800 \\ \mathrm{C} & 0.12556100 & 1.71063500 & -0.42671200 \\ \mathrm{~N} & 1.74869000 & 0.38713700 & 0.49551600 \\ \mathrm{C} & 2.29402600 & 1.62801500 & 0.23724200 \\ \mathrm{C} & 1.30864600 & 2.48016900 & -0.32939700 \\ \mathrm{O} & -1.02047800 & 2.14061700 & -0.90176800 \\ \mathrm{C} & 3.59142600 & 2.10380700 & 0.45784500 \\ \mathrm{C} & 1.60671200 & 3.80908200 & -0.67041400 \\ \mathrm{C} & 3.86528700 & 3.42155600 & 0.11315800 \\ \mathrm{C} & 2.88885100 & 4.27267700 & -0.44553400 \\ \mathrm{H} & 4.36358400 & 1.47373900 & 0.88022000 \\ \mathrm{H} & 4.86533000 & 3.80692300 & 0.27811600 \\ \mathrm{H} & 3.15283600 & 5.29243800 & -0.69809000 \\ \mathrm{H} & 0.83967400 & 4.44268800 & -1.09914900 \\ \mathrm{C} & 2.51786300 & -0.74849900 & 1.01690000 \\ \mathrm{C} & 3.28239700 & -1.50594900 & -0.07080600 \\ \mathrm{H} & 1.82126900 & -1.42784500 & 1.50461100 \\ \mathrm{H} & 3.20219200 & -0.35271900 & 1.77208100 \\ \mathrm{H} & 2.58686000 & -1.95215500 & -0.78397600 \\ \mathrm{H} & 3.97364500 & -0.85607300 & -0.61348700 \\ \mathrm{H} & 3.85679500 & -2.31706900 & 0.38437000\end{array}$


$\mathrm{S}_{1}$ NHxInd (IRC point 40 toward isomer 2), gas phase

$\begin{array}{lrrr}\mathrm{C} & -2.04858700 & -2.37612900 & 0.24950500 \\ \mathrm{C} & -2.75538100 & -3.55108200 & 0.40875600 \\ \mathrm{C} & -4.09989700 & -3.57555600 & 0.00562600 \\ \mathrm{C} & -4.72832000 & -2.44082400 & -0.54944700 \\ \mathrm{C} & -4.03380700 & -1.25395000 & -0.71616000 \\ \mathrm{C} & -2.68340400 & -1.2241500 & -0.31372100 \\ \mathrm{H} & -2.28151900 & -4.42784200 & 0.83483400 \\ \mathrm{H} & -4.67410900 & -4.48763700 & 0.12305700 \\ \mathrm{H} & -5.76809300 & -2.50461200 & -0.84748700 \\ \mathrm{H} & -4.49774600 & -0.37206600 & -1.14180000 \\ \mathrm{C} & -0.65444600 & -1.99059000 & 0.54970000 \\ \mathrm{~N} & -1.83045600 & -0.1811600 & -0.38730200 \\ \mathrm{C} & -0.60315800 & -0.58293800 & 0.10992000 \\ \mathrm{C} & 0.39405300 & 0.42391000 & 0.07170800 \\ \mathrm{H} & -1.64915100 & 1.42572100 & -0.85381800 \\ \mathrm{O} & 0.23149900 & -2.70813700 & 1.04783700 \\ \mathrm{C} & 0.13010300 & 1.73506600 & -0.43480100 \\ \mathrm{~N} & 1.73124900 & 0.39522400 & 0.48377300 \\ \mathrm{C} & 2.29612200 & 1.63139300 & 0.23650300 \\ \mathrm{C} & 1.32212900 & 2.49375300 & -0.33011900 \\ \mathrm{O} & -1.00864700 & 2.19767000 & -0.91638100 \\ \mathrm{C} & 3.59720000 & 2.08952200 & 0.46591000 \\ \mathrm{C} & 1.63600500 & 3.81852000 & -0.66489800 \\ \mathrm{C} & 3.88918100 & 3.40659200 & 0.12683400 \\ \mathrm{C} & 2.92457000 & 4.26737800 & -0.43261300 \\ \mathrm{H} & 4.36010900 & 1.44927200 & 0.88984300 \\ \mathrm{H} & 4.89255500 & 3.78011600 & 0.29762800 \\ \mathrm{H} & 3.19933300 & 5.28530700 & -0.68139400 \\ \mathrm{H} & 0.87900100 & 4.46312500 & -1.09498300 \\ \mathrm{C} & 2.49118300 & -0.74161500 & 1.01401600 \\ \mathrm{C} & 3.25738400 & -1.50717900 & -0.06770300 \\ \mathrm{H} & 1.79183300 & -1.41601600 & 1.50349700 \\ \mathrm{H} & 3.17591200 & -0.34310600 & 1.76717500 \\ \mathrm{H} & 2.56377700 & -1.95601000 & -0.78089200 \\ \mathrm{H} & 3.95179300 & -0.86108600 & -0.61066800 \\ \mathrm{H} & 3.82830600 & -2.31647200 & 0.39487700\end{array}$


$\mathrm{S}_{1}$ NHxInd (IRC point 60 toward isomer 2), gas phase

$\begin{array}{lrrr}\mathrm{C} & -2.06159400 & -2.39285900 & 0.25368600 \\ \mathrm{C} & -2.76505100 & -3.57371300 & 0.41595600 \\ \mathrm{C} & -4.11153600 & -3.60451100 & 0.01438300 \\ \mathrm{C} & -4.73343000 & -2.48028800 & -0.53610800 \\ \mathrm{C} & -4.02593500 & -1.28263900 & -0.70304300 \\ \mathrm{C} & -2.69764900 & -1.26135000 & -0.30365000 \\ \mathrm{H} & -2.28775400 & -4.44895200 & 0.84175500 \\ \mathrm{H} & -4.68108800 & -4.51935800 & 0.13419800 \\ \mathrm{H} & -5.77290000 & -2.53606200 & -0.83695300 \\ \mathrm{H} & -4.49138700 & -0.40195500 & -1.13042300 \\ \mathrm{C} & -0.66810000 & -2.00067200 & 0.54099800 \\ \mathrm{~N} & -1.81386200 & -0.18979400 & -0.38031200 \\ \mathrm{C} & -0.62800600 & -0.57435400 & 0.09251900 \\ \mathrm{C} & 0.38579900 & 0.46029500 & 0.05098000 \\ \mathrm{H} & -1.64977200 & 1.47108700 & -0.87190500 \\ \mathrm{O} & 0.23816800 & -2.69366500 & 1.02758500 \\ \mathrm{C} & 0.13241700 & 1.74859700 & -0.44160000 \\ \mathrm{~N} & 1.72320600 & 0.41633300 & 0.47004300 \\ \mathrm{C} & 2.30644100 & 1.64885600 & 0.23695100 \\ \mathrm{C} & 1.33707600 & 2.51259900 & -0.33058300 \\ \mathrm{O} & -1.00459100 & 2.23083900 & -0.92906900 \\ \mathrm{C} & 3.61066800 & 2.08826600 & 0.47532800 \\ \mathrm{C} & 1.66278700 & 3.82476700 & -0.65978700 \\ \mathrm{C} & 3.92239900 & 3.40597900 & 0.13950900 \\ \mathrm{C} & 2.96535900 & 4.26422400 & -0.41935100 \\ \mathrm{H} & 4.36301000 & 1.43814800 & 0.90347400 \\ \mathrm{H} & 4.92734600 & 3.77089200 & 0.31489800 \\ \mathrm{H} & 3.24294200 & 5.28167600 & -0.66781400 \\ \mathrm{H} & 0.91792200 & 4.48101700 & -1.09304700 \\ \mathrm{C} & 2.46278200 & -0.72493900 & 1.01330700 \\ \mathrm{C} & 3.21876000 & -1.50991000 & -0.06546100 \\ \mathrm{H} & 1.75193300 & -1.38803600 & 1.50317000 \\ \mathrm{H} & 3.15563500 & -0.32762800 & 1.75863000 \\ \mathrm{H} & 2.52008100 & -1.95286200 & -0.77633600 \\ \mathrm{H} & 3.92451000 & -0.87613600 & -0.60761100 \\ \mathrm{H} & 3.77373700 & -2.32313400 & 0.40823400\end{array}$


$\mathrm{S}_{1}$ NHxInd (IRC point 80 toward isomer 2), gas phase

$\begin{array}{lrrr}\mathrm{C} & -2.06159400 & -2.39285900 & 0.25368600 \\ \mathrm{C} & -2.76505100 & -3.57371300 & 0.41595600 \\ \mathrm{C} & -4.11153600 & -3.60451100 & 0.01438300 \\ \mathrm{C} & -4.73343000 & -2.48028800 & -0.53610800 \\ \mathrm{C} & -4.02593500 & -1.28263900 & -0.70304300 \\ \mathrm{C} & -2.69764900 & -1.26135000 & -0.30365000 \\ \mathrm{H} & -2.28775400 & -4.44895200 & 0.84175500 \\ \mathrm{H} & -4.68108800 & -4.51935800 & 0.13419800 \\ \mathrm{H} & -5.77290000 & -2.53606200 & -0.83695300 \\ \mathrm{H} & -4.49138700 & -0.40195500 & -1.13042300 \\ \mathrm{C} & -0.66810000 & -2.00067200 & 0.54099800 \\ \mathrm{~N} & -1.81386200 & -0.18979400 & -0.38031200 \\ \mathrm{C} & -0.62800600 & -0.57435400 & 0.09251900 \\ \mathrm{C} & 0.38579900 & 0.46029500 & 0.05098000 \\ \mathrm{H} & -1.64977200 & 1.47108700 & -0.87190500 \\ \mathrm{O} & 0.23816800 & -2.69366500 & 1.02758500 \\ \mathrm{C} & 0.13241700 & 1.74859700 & -0.44160000 \\ \mathrm{~N} & 1.72320600 & 0.41633300 & 0.47004300 \\ \mathrm{C} & 2.30644100 & 1.64885600 & 0.23695100 \\ \mathrm{C} & 1.33707600 & 2.51259900 & -0.33058300 \\ \mathrm{O} & -1.00459100 & 2.23083900 & -0.92906900 \\ \mathrm{C} & 3.61066800 & 2.08826600 & 0.47532800 \\ \mathrm{C} & 1.66278700 & 3.82476700 & -0.65978700 \\ \mathrm{C} & 3.92239900 & 3.40597900 & 0.13950900 \\ \mathrm{C} & 2.96535900 & 4.26422400 & -0.41935100 \\ \mathrm{H} & 4.36301000 & 1.43814800 & 0.90347400 \\ \mathrm{H} & 4.92734600 & 3.77089200 & 0.31489800 \\ \mathrm{H} & 3.24294200 & 5.28167600 & -0.66781400 \\ \mathrm{H} & 0.91792200 & 4.48101700 & -1.09304700 \\ \mathrm{C} & 2.46278200 & -0.72493900 & 1.01330700 \\ \mathrm{C} & 3.21876000 & -1.50991000 & -0.06546100 \\ \mathrm{H} & 1.75193300 & -1.38803600 & 1.50317000 \\ \mathrm{H} & 3.15563500 & -0.32762800 & 1.75863000 \\ \mathrm{H} & 2.52008100 & -1.95286200 & -0.77633600 \\ \mathrm{H} & 3.92451000 & -0.87613600 & -0.60761100 \\ \mathrm{H} & 3.77373700 & -2.32313400 & 0.40823400 \\ & & & \end{array}$




$\begin{array}{lrrr}\mathrm{C} & -2.04965300 & -2.39201300 & 0.24817200 \\ \mathrm{C} & -2.73001800 & -3.58502700 & 0.42727300 \\ \mathrm{C} & -4.08137100 & -3.63994200 & 0.04991900 \\ \mathrm{C} & -4.72705200 & -2.52689200 & -0.49407800 \\ \mathrm{C} & -4.04033100 & -1.31732900 & -0.67786200 \\ \mathrm{C} & -2.70767500 & -1.27114700 & -0.30106100 \\ \mathrm{H} & -2.22875900 & -4.44927000 & 0.84805000 \\ \mathrm{H} & -4.63506100 & -4.56267400 & 0.18302900 \\ \mathrm{H} & -5.77049400 & -2.59813500 & -0.77763500 \\ \mathrm{H} & -4.52938000 & -0.44740800 & -1.10105200 \\ \mathrm{C} & -0.65968100 & -1.98456900 & 0.51091800 \\ \mathrm{~N} & -1.83321100 & -0.18212800 & -0.39173000 \\ \mathrm{C} & -0.64325000 & -0.55503900 & 0.06582500 \\ \mathrm{C} & 0.37269400 & 0.48613300 & 0.02572400 \\ \mathrm{H} & -1.64724800 & 1.51068400 & -0.91178400 \\ \mathrm{O} & 0.26360600 & -2.66981300 & 0.97718800 \\ \mathrm{C} & 0.13702600 & 1.77128000 & -0.46683700 \\ \mathrm{~N} & 1.70403600 & 0.43325900 & 0.46892700 \\ \mathrm{C} & 2.30206500 & 1.65858000 & 0.24757800 \\ \mathrm{C} & 1.34942000 & 2.53086200 & -0.33616200 \\ \mathrm{O} & -0.99324300 & 2.25945600 & -0.96992000 \\ \mathrm{C} & 3.60929900 & 2.07849000 & 0.50723800 \\ \mathrm{C} & 1.70125300 & 3.83645900 & -0.65860900 \\ \mathrm{C} & 3.94658000 & 3.39109400 & 0.17785500 \\ \mathrm{C} & 3.00853000 & 4.25873600 & -0.39555200 \\ \mathrm{H} & 4.34465500 & 1.41622000 & 0.94579500 \\ \mathrm{H} & 4.95320200 & 3.74256700 & 0.36929100 \\ \mathrm{H} & 3.30148700 & 5.27290900 & -0.63984500 \\ \mathrm{H} & 0.97609900 & 4.50573700 & -1.10515200 \\ \mathrm{C} & 2.42646500 & -0.71316900 & 1.02069100 \\ \mathrm{C} & 3.11236800 & -1.55478400 & -0.06178300 \\ \mathrm{H} & 1.71572100 & -1.34107200 & 1.55475800 \\ \mathrm{H} & 3.15901300 & -0.31550100 & 1.72552900 \\ \mathrm{H} & 2.37189300 & -1.99299600 & -0.73085800 \\ \mathrm{H} & 3.81950600 & -0.96036400 & -0.64533700 \\ \mathrm{H} & 3.65443200 & -2.37408800 & 0.41596600\end{array}$


$\mathrm{S}_{1}$ NHxInd (IRC point 1 toward isomer 1), gas phase

$\begin{array}{lrrr}\mathrm{C} & -2.04121769 & -2.34095912 & 0.23879069 \\ \mathrm{C} & -2.75313769 & -3.51226812 & 0.39490669 \\ \mathrm{C} & -4.09255469 & -3.53629212 & -0.00645031 \\ \mathrm{C} & -4.72326369 & -2.38822112 & -0.56572731 \\ \mathrm{C} & -4.04382369 & -1.20830312 & -0.73471331 \\ \mathrm{C} & -2.67398269 & -1.16832412 & -0.32960831 \\ \mathrm{H} & -2.27550969 & -4.38670412 & 0.82127869 \\ \mathrm{H} & -4.67157969 & -4.44512412 & 0.10832869 \\ \mathrm{H} & -5.76350769 & -2.45704512 & -0.86160731 \\ \mathrm{H} & -4.51320969 & -0.32950112 & -1.15949131 \\ \mathrm{C} & -0.64115269 & -1.98796212 & 0.55676469 \\ \mathrm{~N} & -1.82261669 & -0.16083512 & -0.39045731 \\ \mathrm{C} & -0.55184869 & -0.60698412 & 0.13747069 \\ \mathrm{C} & 0.42061631 & 0.38448888 & 0.09683969 \\ \mathrm{H} & -1.68357269 & 1.07343788 & -0.75013331 \\ \mathrm{O} & 0.21553931 & -2.73870812 & 1.06423669 \\ \mathrm{C} & 0.10773331 & 1.71128188 & -0.43167031 \\ \mathrm{~N} & 1.75184431 & 0.38624088 & 0.49712769 \\ \mathrm{C} & 2.29156631 & 1.63410388 & 0.23379769 \\ \mathrm{C} & 1.30540431 & 2.48375388 & -0.33158831 \\ \mathrm{O} & -1.03715369 & 2.07507388 & -0.88590131 \\ \mathrm{C} & 3.58750231 & 2.10555388 & 0.45571269 \\ \mathrm{C} & 1.60593231 & 3.80960488 & -0.67084731 \\ \mathrm{C} & 3.86455731 & 3.42514288 & 0.11177769 \\ \mathrm{C} & 2.89065631 & 4.27503588 & -0.44570531 \\ \mathrm{H} & 4.35781031 & 1.47329988 & 0.87812669 \\ \mathrm{H} & 4.86524331 & 3.80856588 & 0.27774869 \\ \mathrm{H} & 3.15368631 & 5.29494088 & -0.69850031 \\ \mathrm{H} & 0.83926931 & 4.44395788 & -1.09963931 \\ \mathrm{C} & 2.52117831 & -0.74838812 & 1.01770469 \\ \mathrm{C} & 3.28412331 & -1.50541612 & -0.07105031 \\ \mathrm{H} & 1.82293731 & -1.42874312 & 1.50259369 \\ \mathrm{H} & 3.20514431 & -0.35524012 & 1.77473569 \\ \mathrm{H} & 2.58655631 & -1.95124912 & -0.78252231 \\ \mathrm{H} & 3.97494431 & -0.85628012 & -0.61525431 \\ \mathrm{H} & 3.85866031 & -2.31705512 & 0.38304269\end{array}$


$\mathrm{S}_{1}$ NHxInd (IRC point 20 toward isomer 1), gas phase

$\begin{array}{lrrr}\mathrm{C} & -2.04402200 & -2.34045300 & 0.23774100 \\ \mathrm{C} & -2.75044500 & -3.50792500 & 0.39416500 \\ \mathrm{C} & -4.09483800 & -3.53434800 & -0.00777000 \\ \mathrm{C} & -4.72981700 & -2.39116700 & -0.56678700 \\ \mathrm{C} & -4.05496900 & -1.20988500 & -0.73758300 \\ \mathrm{C} & -2.68985000 & -1.18216400 & -0.33005000 \\ \mathrm{H} & -2.27075900 & -4.38077000 & 0.82080800 \\ \mathrm{H} & -4.67079300 & -4.44453700 & 0.10891500 \\ \mathrm{H} & -5.76964800 & -2.46319000 & -0.86155800 \\ \mathrm{H} & -4.52405000 & -0.33141100 & -1.16262100 \\ \mathrm{C} & -0.63180900 & -1.98099700 & 0.55758800 \\ \mathrm{~N} & -1.82676900 & -0.19786900 & -0.37977600 \\ \mathrm{C} & -0.53271000 & -0.61633100 & 0.14671600 \\ \mathrm{C} & 0.42352900 & 0.38350100 & 0.09835500 \\ \mathrm{H} & -1.89958800 & 0.78853800 & -0.71920100 \\ \mathrm{O} & 0.21041300 & -2.74352500 & 1.06425800 \\ \mathrm{C} & 0.09325400 & 1.72955900 & -0.44154200 \\ \mathrm{~N} & 1.74358300 & 0.38438700 & 0.49423000 \\ \mathrm{C} & 2.29552200 & 1.64296900 & 0.23153700 \\ \mathrm{C} & 1.31631400 & 2.48851600 & -0.33001100 \\ \mathrm{O} & -1.00983100 & 2.11391400 & -0.89054700 \\ \mathrm{C} & 3.58694800 & 2.10524500 & 0.45566800 \\ \mathrm{C} & 1.60846400 & 3.80752100 & -0.66950300 \\ \mathrm{C} & 3.86762900 & 3.43038100 & 0.11097500 \\ \mathrm{C} & 2.89730000 & 4.27625600 & -0.44414800 \\ \mathrm{H} & 4.35714500 & 1.47273800 & 0.87838300 \\ \mathrm{H} & 4.86885700 & 3.81155600 & 0.27784300 \\ \mathrm{H} & 3.15881600 & 5.29664900 & -0.69753400 \\ \mathrm{H} & 0.83728800 & 4.43667900 & -1.09814400 \\ \mathrm{C} & 2.51793200 & -0.74697800 & 1.01681300 \\ \mathrm{C} & 3.28368600 & -1.50422400 & -0.07120200 \\ \mathrm{H} & 1.82327500 & -1.43002900 & 1.50326800 \\ \mathrm{H} & 3.20182900 & -0.34896500 & 1.77135300 \\ \mathrm{H} & 2.58663800 & -1.95142700 & -0.78231900 \\ \mathrm{H} & 3.97404400 & -0.85496500 & -0.61549300 \\ \mathrm{H} & 3.85840600 & -2.31459100 & 0.38505800 \\ & & & \end{array}$


$\mathrm{S}_{1}$ NHxInd (IRC point 40 toward isomer 1), gas phase

$\begin{array}{lrrr}\mathrm{C} & -2.04911100 & -2.35727200 & 0.24139300 \\ \mathrm{C} & -2.75216900 & -3.52836400 & 0.39959500 \\ \mathrm{C} & -4.09869600 & -3.56344500 & 0.00012300 \\ \mathrm{C} & -4.74400800 & -2.42748000 & -0.55942700 \\ \mathrm{C} & -4.07283000 & -1.24330400 & -0.73202500 \\ \mathrm{C} & -2.70554500 & -1.20773900 & -0.32631400 \\ \mathrm{H} & -2.26942300 & -4.39952100 & 0.82630100 \\ \mathrm{H} & -4.66691100 & -4.47803000 & 0.12061800 \\ \mathrm{H} & -5.78394100 & -2.50578100 & -0.85223700 \\ \mathrm{H} & -4.54589400 & -0.36708100 & -1.15756400 \\ \mathrm{C} & -0.63439600 & -1.98605000 & 0.55830600 \\ \mathrm{~N} & -1.84466100 & -0.21564000 & -0.37868200 \\ \mathrm{C} & -0.53948400 & -0.61479200 & 0.14430900 \\ \mathrm{C} & 0.41011500 & 0.40178000 & 0.08667300 \\ \mathrm{H} & -1.94716400 & 0.75724000 & -0.72110000 \\ \mathrm{O} & 0.20199700 & -2.75459800 & 1.06537000 \\ \mathrm{C} & 0.09813600 & 1.76414200 & -0.45164100 \\ \mathrm{~N} & 1.73087800 & 0.39626400 & 0.48120500 \\ \mathrm{C} & 2.30203200 & 1.65156800 & 0.22751600 \\ \mathrm{C} & 1.33530000 & 2.50801600 & -0.33231700 \\ \mathrm{O} & -0.99035100 & 2.17447200 & -0.90279800 \\ \mathrm{C} & 3.59767100 & 2.10094200 & 0.45883800 \\ \mathrm{C} & 1.64226500 & 3.82526900 & -0.66565000 \\ \mathrm{C} & 3.89475500 & 3.42561900 & 0.12063000 \\ \mathrm{C} & 2.93532500 & 4.28248800 & -0.43476900 \\ \mathrm{H} & 4.36034800 & 1.45979600 & 0.88230100 \\ \mathrm{H} & 4.89934300 & 3.79512200 & 0.29342300 \\ \mathrm{H} & 3.20639500 & 5.30148100 & -0.68395100 \\ \mathrm{H} & 0.87802100 & 4.46298900 & -1.09412900 \\ \mathrm{C} & 2.49418100 & -0.73840000 & 1.01002500 \\ \mathrm{C} & 3.27290900 & -1.49312700 & -0.07059400 \\ \mathrm{H} & 1.79357900 & -1.42263300 & 1.48641000 \\ \mathrm{H} & 3.16988400 & -0.34436200 & 1.77410700 \\ \mathrm{H} & 2.58480500 & -1.94284500 & -0.78883300 \\ \mathrm{H} & 3.96628500 & -0.84146900 & -0.60803500 \\ \mathrm{H} & 3.84597600 & -2.30120800 & 0.39169900 \\ & & & \end{array}$




$\begin{array}{lrrr}\mathrm{C} & -2.04730300 & -2.35302900 & 0.23940400 \\ \mathrm{C} & -2.72841100 & -3.53531100 & 0.40763800 \\ \mathrm{C} & -4.07583900 & -3.59494300 & 0.01650600 \\ \mathrm{C} & -4.74009100 & -2.47085400 & -0.54411400 \\ \mathrm{C} & -4.08890400 & -1.27580300 & -0.72624100 \\ \mathrm{C} & -2.71968200 & -1.21396600 & -0.32920700 \\ \mathrm{H} & -2.22623900 & -4.39457300 & 0.83597400 \\ \mathrm{H} & -4.62931000 & -4.51755800 & 0.14415200 \\ \mathrm{H} & -5.78029600 & -2.56576100 & -0.83104700 \\ \mathrm{H} & -4.58104700 & -0.41096300 & -1.15347200 \\ \mathrm{C} & -0.63814600 & -1.96691100 & 0.54996900 \\ \mathrm{~N} & -1.86774400 & -0.21108600 & -0.38778600 \\ \mathrm{C} & -0.55599300 & -0.59620900 & 0.13286200 \\ \mathrm{C} & 0.39495700 & 0.42019600 & 0.07615600 \\ \mathrm{H} & -1.98258900 & 0.75714600 & -0.73142200 \\ \mathrm{O} & 0.20291400 & -2.73102400 & 1.05646800 \\ \mathrm{C} & 0.09631400 & 1.78725500 & -0.46013900 \\ \mathrm{~N} & 1.71328600 & 0.40249300 & 0.47464200 \\ \mathrm{C} & 2.29808100 & 1.65171300 & 0.22589600 \\ \mathrm{C} & 1.34344700 & 2.52083800 & -0.33523000 \\ \mathrm{O} & -0.98972300 & 2.20124300 & -0.91098400 \\ \mathrm{C} & 3.59926800 & 2.07883900 & 0.46603500 \\ \mathrm{C} & 1.67509900 & 3.83491200 & -0.66036700 \\ \mathrm{C} & 3.91956800 & 3.39961700 & 0.13578800 \\ \mathrm{C} & 2.97470400 & 4.27147500 & -0.42043100 \\ \mathrm{H} & 4.34856900 & 1.42301300 & 0.89059700 \\ \mathrm{H} & 4.92863500 & 3.75336100 & 0.31517300 \\ \mathrm{H} & 3.26118900 & 5.28761900 & -0.66408400 \\ \mathrm{H} & 0.92511900 & 4.48863700 & -1.09003800 \\ \mathrm{C} & 2.47010900 & -0.73464400 & 1.00621000 \\ \mathrm{C} & 3.25289800 & -1.48764000 & -0.07243200 \\ \mathrm{H} & 1.76644400 & -1.41889700 & 1.47694300 \\ \mathrm{H} & 3.14252600 & -0.34295200 & 1.77433100 \\ \mathrm{H} & 2.56751300 & -1.93217800 & -0.79647300 \\ \mathrm{H} & 3.95182700 & -0.83666900 & -0.60342500 \\ \mathrm{H} & 3.82013500 & -2.29959300 & 0.39023900\end{array}$




\section{wB97X-D/6-311G(d,p) geometries (in $\AA$ )}

$\mathrm{S}_{0}$ Ind (IRC point 1 toward isomer 2), gas phase

$\begin{array}{lrrr}\mathrm{C} & 2.94950699 & 0.66572649 & -0.00000124 \\ \mathrm{C} & 4.25003899 & 1.14006849 & 0.00000576 \\ \mathrm{C} & 5.28835499 & 0.21439949 & -0.00004124 \\ \mathrm{C} & 5.00488099 & -1.15176451 & -0.00009224 \\ \mathrm{C} & 3.69843099 & -1.63756051 & -0.00009924 \\ \mathrm{C} & 2.66811799 & -0.71160951 & -0.00005324 \\ \mathrm{H} & 4.44138299 & 2.20721049 & 0.00004576 \\ \mathrm{H} & 6.31795699 & 0.55029749 & -0.00003824 \\ \mathrm{H} & 5.82622499 & -1.86000751 & -0.00012824 \\ \mathrm{H} & 3.49242299 & -2.70072451 & -0.00013924 \\ \mathrm{C} & 1.65374499 & 1.36543749 & 0.00003976 \\ \mathrm{~N} & 1.28863199 & -0.95749551 & -0.00004924 \\ \mathrm{C} & 0.66075399 & 0.22091049 & 0.00000276 \\ \mathrm{C} & -0.71339001 & 0.24466349 & 0.00001676 \\ \mathrm{H} & 0.33789399 & -1.83936051 & -0.00007624 \\ \mathrm{O} & 1.37283799 & 2.54020249 & 0.00008876 \\ \mathrm{C} & -1.41510601 & -0.99546551 & -0.00002624 \\ \mathrm{~N} & -1.62855701 & 1.28623249 & 0.00006676 \\ \mathrm{H} & -1.38141501 & 2.25977849 & 0.00010376 \\ \mathrm{C} & -2.88982701 & 0.75100449 & 0.00005676 \\ \mathrm{C} & -2.81256201 & -0.66324651 & -0.00000024 \\ \mathrm{O} & -0.81897301 & -2.12791351 & -0.00007724 \\ \mathrm{C} & -4.12930501 & 1.39711549 & 0.00009376 \\ \mathrm{C} & -3.97648501 & -1.43867951 & -0.00002124 \\ \mathrm{C} & -5.26267001 & 0.60776649 & 0.00007276 \\ \mathrm{C} & -5.19709101 & -0.79829951 & 0.00001576 \\ \mathrm{H} & -4.19805301 & 2.47841849 & 0.00013776 \\ \mathrm{H} & -6.23497701 & 1.08786949 & 0.00010076 \\ \mathrm{H} & -6.11454801 & -1.37381351 & 0.00000076 \\ \mathrm{H} & -3.90355701 & -2.51974451 & -0.00006524\end{array}$


$\mathrm{S}_{0}$ Ind (IRC point 20 toward isomer 2), gas phase

$\begin{array}{lrrr}\mathrm{C} & 2.96593700 & 0.68212100 & -0.00000100 \\ \mathrm{C} & 4.26325400 & 1.15078800 & 0.00000600 \\ \mathrm{C} & 5.29579300 & 0.21409900 & -0.00004100 \\ \mathrm{C} & 5.01103400 & -1.14980000 & -0.00009200 \\ \mathrm{C} & 3.70036100 & -1.62723700 & -0.00009900 \\ \mathrm{C} & 2.68638400 & -0.68937500 & -0.00005200 \\ \mathrm{H} & 4.46516400 & 2.21590500 & 0.00004600 \\ \mathrm{H} & 6.32672700 & 0.54609300 & -0.00003800 \\ \mathrm{H} & 5.83109200 & -1.85934900 & -0.00012800 \\ \mathrm{H} & 3.47911400 & -2.68736900 & -0.00013900 \\ \mathrm{C} & 1.66012500 & 1.37086500 & 0.00003900 \\ \mathrm{~N} & 1.30972300 & -0.94757000 & -0.00004900 \\ \mathrm{C} & 0.67853100 & 0.20313200 & 0.00000200 \\ \mathrm{C} & -0.71900900 & 0.22620800 & 0.00001600 \\ \mathrm{H} & 0.11776600 & -2.01562500 & -0.00008100 \\ \mathrm{O} & 1.37126100 & 2.54070500 & 0.00008900 \\ \mathrm{C} & -1.42723100 & -0.97958200 & -0.00002600 \\ \mathrm{~N} & -1.62650900 & 1.27982400 & 0.00006600 \\ \mathrm{H} & -1.37088900 & 2.25131100 & 0.00010400 \\ \mathrm{C} & -2.89043600 & 0.75614500 & 0.00005700 \\ \mathrm{C} & -2.81284700 & -0.65841900 & 0.00000000 \\ \mathrm{O} & -0.87569000 & -2.16915000 & -0.00007800 \\ \mathrm{C} & -4.13237200 & 1.40043900 & 0.00009400 \\ \mathrm{C} & -3.97691100 & -1.43795300 & -0.00002100 \\ \mathrm{C} & -5.26346100 & 0.61045900 & 0.00007300 \\ \mathrm{C} & -5.19504900 & -0.79769500 & 0.00001600 \\ \mathrm{H} & -4.20309500 & 2.48170400 & 0.00013800 \\ \mathrm{H} & -6.23670100 & 1.08841400 & 0.00010100 \\ \mathrm{H} & -6.11252400 & -1.37349700 & 0.00000100 \\ \mathrm{H} & -3.90395400 & -2.51886400 & -0.00006500\end{array}$


$\mathrm{S}_{0}$ Ind (IRC point 40 toward isomer 2), gas phase

$\begin{array}{lrrr}\mathrm{C} & 2.97914000 & 0.69630800 & 0.00000000 \\ \mathrm{C} & 4.27800900 & 1.16955600 & 0.00000600 \\ \mathrm{C} & 5.31577000 & 0.23673700 & -0.00004000 \\ \mathrm{C} & 5.03774100 & -1.12832000 & -0.00009200 \\ \mathrm{C} & 3.72813700 & -1.60815200 & -0.00009800 \\ \mathrm{C} & 2.70853200 & -0.67710900 & -0.00005200 \\ \mathrm{H} & 4.47885000 & 2.23494700 & 0.00004700 \\ \mathrm{H} & 6.34510200 & 0.57395000 & -0.00003700 \\ \mathrm{H} & 5.85983300 & -1.83547800 & -0.00012800 \\ \mathrm{H} & 3.50893200 & -2.66871100 & -0.00013800 \\ \mathrm{C} & 1.66034700 & 1.36823800 & 0.00003900 \\ \mathrm{~N} & 1.33041700 & -0.95912400 & -0.00005000 \\ \mathrm{C} & 0.68775300 & 0.17946600 & 0.00000100 \\ \mathrm{C} & -0.71848700 & 0.19143300 & 0.00001500 \\ \mathrm{H} & 0.03395700 & -2.11363900 & -0.00008400 \\ \mathrm{O} & 1.35754100 & 2.53402900 & 0.00008900 \\ \mathrm{C} & -1.45082000 & -0.99701100 & -0.00002600 \\ \mathrm{~N} & -1.61276000 & 1.26026500 & 0.00006500 \\ \mathrm{H} & -1.33779000 & 2.22671900 & 0.00010200 \\ \mathrm{C} & -2.88683800 & 0.76113100 & 0.00005700 \\ \mathrm{C} & -2.82735300 & -0.65292500 & 0.00000000 \\ \mathrm{O} & -0.95116000 & -2.21710700 & -0.00007900 \\ \mathrm{C} & -4.12599000 & 1.41412200 & 0.00009500 \\ \mathrm{C} & -3.99460900 & -1.42659200 & -0.00002000 \\ \mathrm{C} & -5.26262700 & 0.63086000 & 0.00007400 \\ \mathrm{C} & -5.20729000 & -0.77837700 & 0.00001700 \\ \mathrm{H} & -4.19219100 & 2.49568300 & 0.00013900 \\ \mathrm{H} & -6.23211700 & 1.11644700 & 0.00010200 \\ \mathrm{H} & -6.12966600 & -1.34632900 & 0.00000200 \\ \mathrm{H} & -3.92581200 & -2.50774100 & -0.00006400\end{array}$


$\mathrm{S}_{0}$ Ind (IRC point 60 toward isomer 2), gas phase

$\begin{array}{lrrr}\mathrm{C} & 2.97467100 & 0.69635400 & 0.00000000 \\ \mathrm{C} & 4.26481700 & 1.19315700 & 0.00000700 \\ \mathrm{C} & 5.31920100 & 0.27938100 & -0.00003900 \\ \mathrm{C} & 5.06364400 & -1.08991200 & -0.00009100 \\ \mathrm{C} & 3.76188400 & -1.59274800 & -0.00009800 \\ \mathrm{C} & 2.72446100 & -0.68118300 & -0.00005200 \\ \mathrm{H} & 4.44564800 & 2.26213900 & 0.00004800 \\ \mathrm{H} & 6.34258600 & 0.63424600 & -0.00003500 \\ \mathrm{H} & 5.89732000 & -1.78339300 & -0.00012600 \\ \mathrm{H} & 3.56290800 & -2.65730000 & -0.00013800 \\ \mathrm{C} & 1.64679600 & 1.34731400 & 0.00003900 \\ \mathrm{~N} & 1.34727300 & -0.98581500 & -0.00005100 \\ \mathrm{C} & 0.69169500 & 0.14400300 & 0.00000000 \\ \mathrm{C} & -0.71596400 & 0.15599400 & 0.00001300 \\ \mathrm{H} & 0.00093200 & -2.16544400 & -0.00008600 \\ \mathrm{O} & 1.32686900 & 2.50877300 & 0.00008800 \\ \mathrm{C} & -1.46491800 & -1.02052300 & -0.00002700 \\ \mathrm{~N} & -1.59419200 & 1.23778100 & 0.00006400 \\ \mathrm{H} & -1.30270100 & 2.19959700 & 0.00010100 \\ \mathrm{C} & -2.87500300 & 0.75912200 & 0.00005700 \\ \mathrm{C} & -2.83737200 & -0.65610100 & 0.00000000 \\ \mathrm{O} & -0.98203900 & -2.25002400 & -0.00008000 \\ \mathrm{C} & -4.10245000 & 1.43434500 & 0.00009500 \\ \mathrm{C} & -4.01983900 & -1.40785900 & -0.00001900 \\ \mathrm{C} & -5.25277600 & 0.67211000 & 0.00007500 \\ \mathrm{C} & -5.22098400 & -0.73805900 & 0.00001800 \\ \mathrm{H} & -4.14831200 & 2.51697000 & 0.00013900 \\ \mathrm{H} & -6.21369700 & 1.17442100 & 0.00010400 \\ \mathrm{H} & -6.15289900 & -1.29027000 & 0.00000500 \\ \mathrm{H} & -3.97197600 & -2.49015200 & -0.00006300\end{array}$


$\mathrm{S}_{0}$ Ind (IRC point 80 toward isomer 2 ), gas phase

$\begin{array}{lrrr}\mathrm{C} & 2.96627200 & 0.69559300 & 0.00000000 \\ \mathrm{C} & 4.24655600 & 1.21649400 & 0.00000800 \\ \mathrm{C} & 5.31733300 & 0.32241000 & -0.00003700 \\ \mathrm{C} & 5.08521800 & -1.05095600 & -0.00008900 \\ \mathrm{C} & 3.79232000 & -1.57772500 & -0.00009800 \\ \mathrm{C} & 2.73755000 & -0.68613600 & -0.00005200 \\ \mathrm{H} & 4.40666400 & 2.28877300 & 0.00004900 \\ \mathrm{H} & 6.33430400 & 0.69523000 & -0.00003200 \\ \mathrm{H} & 5.93088400 & -1.72977400 & -0.00012400 \\ \mathrm{H} & 3.61402300 & -2.64593400 & -0.00013800 \\ \mathrm{C} & 1.62984900 & 1.32457300 & 0.00003800 \\ \mathrm{~N} & 1.36279500 & -1.01365200 & -0.00005200 \\ \mathrm{C} & 0.69474500 & 0.10774900 & -0.00000200 \\ \mathrm{C} & -0.71241300 & 0.12153100 & 0.00001200 \\ \mathrm{H} & -0.02478300 & -2.21023000 & -0.00008700 \\ \mathrm{O} & 1.28989800 & 2.48077300 & 0.00008700 \\ \mathrm{C} & -1.47623400 & -1.04373100 & -0.00002800 \\ \mathrm{~N} & -1.57339500 & 1.21595500 & 0.00006300 \\ \mathrm{H} & -1.26458200 & 2.17264700 & 0.00009900 \\ \mathrm{C} & -2.86007000 & 0.75714800 & 0.00005700 \\ \mathrm{C} & -2.84420500 & -0.65921100 & 0.00000000 \\ \mathrm{O} & -1.00637100 & -2.28020400 & -0.00008100 \\ \mathrm{C} & -4.07481300 & 1.45476000 & 0.00009600 \\ \mathrm{C} & -4.04135100 & -1.38849200 & -0.00001800 \\ \mathrm{C} & -5.23836800 & 0.71386000 & 0.00007700 \\ \mathrm{C} & -5.23024400 & -0.69681900 & 0.00002000 \\ \mathrm{H} & -4.09981100 & 2.53806500 & 0.00013900 \\ \mathrm{H} & -6.19044500 & 1.23270200 & 0.00010600 \\ \mathrm{H} & -6.17159700 & -1.23281500 & 0.00000700 \\ \mathrm{H} & -4.01476600 & -2.47152500 & -0.00006200\end{array}$


$\mathrm{S}_{0}$ Ind (IRC point 100 toward isomer 2), gas phase

$\begin{array}{lrrr}\mathrm{C} & 2.95734900 & 0.69482500 & 0.00000000 \\ \mathrm{C} & 4.22732600 & 1.23957100 & 0.00000900 \\ \mathrm{C} & 5.31403400 & 0.36535500 & -0.00003500 \\ \mathrm{C} & 5.10535200 & -1.01167200 & -0.00008800 \\ \mathrm{C} & 3.82177600 & -1.56217800 & -0.00009700 \\ \mathrm{C} & 2.75021600 & -0.69071100 & -0.00005300 \\ \mathrm{H} & 4.36672100 & 2.31473500 & 0.00005000 \\ \mathrm{H} & 6.32430600 & 0.75592600 & -0.00002900 \\ \mathrm{H} & 5.96273500 & -1.67564200 & -0.00012200 \\ \mathrm{H} & 3.66409400 & -2.63361900 & -0.00013800 \\ \mathrm{C} & 1.61281200 & 1.30175800 & 0.00003700 \\ \mathrm{~N} & 1.37851400 & -1.04103200 & -0.00005400 \\ \mathrm{C} & 0.69774600 & 0.07162600 & -0.00000300 \\ \mathrm{C} & -0.70887100 & 0.08691000 & 0.00001000 \\ \mathrm{H} & -0.05078300 & -2.25454500 & -0.00008900 \\ \mathrm{O} & 1.25325700 & 2.45244900 & 0.00008600 \\ \mathrm{C} & -1.48754700 & -1.06695800 & -0.00002900 \\ \mathrm{~N} & -1.55254700 & 1.19363600 & 0.00006200 \\ \mathrm{H} & -1.22694400 & 2.14500300 & 0.00009700 \\ \mathrm{C} & -2.84464500 & 0.75479900 & 0.00005700 \\ \mathrm{C} & -2.85066300 & -0.66227500 & 0.00000000 \\ \mathrm{O} & -1.03106600 & -2.31024300 & -0.00008200 \\ \mathrm{C} & -4.04611100 & 1.47473700 & 0.00009600 \\ \mathrm{C} & -4.06196200 & -1.36883300 & -0.00001700 \\ \mathrm{C} & -5.22246200 & 0.75541300 & 0.00007800 \\ \mathrm{C} & -5.23810600 & -0.65540000 & 0.00002200 \\ \mathrm{H} & -4.05012600 & 2.55832000 & 0.00014000 \\ \mathrm{H} & -6.16542100 & 1.29061300 & 0.00010800 \\ \mathrm{H} & -6.18867900 & -1.17489900 & 0.00001000 \\ \mathrm{H} & -4.05666900 & -2.45218500 & -0.00006100\end{array}$




$\begin{array}{lrrr}\mathrm{C} & 2.95143600 & 0.69449800 & 0.00000000 \\ \mathrm{C} & 4.21401600 & 1.25566900 & 0.00001000 \\ \mathrm{C} & 5.31150400 & 0.39530400 & -0.00003400 \\ \mathrm{C} & 5.11907400 & -0.98403700 & -0.00008600 \\ \mathrm{C} & 3.84222200 & -1.55084900 & -0.00009600 \\ \mathrm{C} & 2.75935600 & -0.69340700 & -0.00005300 \\ \mathrm{H} & 4.33907700 & 2.33259100 & 0.00005100 \\ \mathrm{H} & 6.31697400 & 0.79805600 & -0.00002800 \\ \mathrm{H} & 5.98442300 & -1.63760400 & -0.00012000 \\ \mathrm{H} & 3.69877000 & -2.62428500 & -0.00013700 \\ \mathrm{C} & 1.60148000 & 1.28611900 & 0.00003600 \\ \mathrm{~N} & 1.39007300 & -1.05952200 & -0.00005400 \\ \mathrm{C} & 0.70006500 & 0.04660600 & -0.00000400 \\ \mathrm{C} & -0.70651300 & 0.06225500 & 0.00000900 \\ \mathrm{H} & -0.07155200 & -2.28782200 & -0.00009000 \\ \mathrm{O} & 1.22911700 & 2.43300800 & 0.00008500 \\ \mathrm{C} & -1.49601200 & -1.08331000 & -0.00002900 \\ \mathrm{~N} & -1.53815300 & 1.17749700 & 0.00006200 \\ \mathrm{H} & -1.20095700 & 2.12502300 & 0.00009600 \\ \mathrm{C} & -2.83393300 & 0.75295300 & 0.00005600 \\ \mathrm{C} & -2.85539500 & -0.66428900 & 0.00000000 \\ \mathrm{O} & -1.05074600 & -2.33196000 & -0.00008300 \\ \mathrm{C} & -4.02587800 & 1.48836200 & 0.00009700 \\ \mathrm{C} & -4.07621700 & -1.35495600 & -0.00001700 \\ \mathrm{C} & -5.21090600 & 0.78418600 & 0.00007900 \\ \mathrm{C} & -5.24321700 & -0.62649300 & 0.00002300 \\ \mathrm{H} & -4.01529600 & 2.57189800 & 0.00014000 \\ \mathrm{H} & -6.14733500 & 1.33071200 & 0.00011000 \\ \mathrm{H} & -6.20008000 & -1.13433600 & 0.00001100 \\ \mathrm{H} & -4.08567500 & -2.43828400 & -0.00006000\end{array}$


$\mathrm{S}_{0}$ Ind (IRC point 1 toward isomer 1), gas phase

$\begin{array}{lrrr}\mathrm{C} & 2.94950699 & 0.66572649 & -0.00000124 \\ \mathrm{C} & 4.25003899 & 1.14006849 & 0.00000576 \\ \mathrm{C} & 5.28835499 & 0.21439949 & -0.00004124 \\ \mathrm{C} & 5.00488099 & -1.15176451 & -0.00009224 \\ \mathrm{C} & 3.69843099 & -1.63756051 & -0.00009924 \\ \mathrm{C} & 2.66811799 & -0.71160951 & -0.00005324 \\ \mathrm{H} & 4.44138299 & 2.20721049 & 0.00004576 \\ \mathrm{H} & 6.31795699 & 0.55029749 & -0.00003824 \\ \mathrm{H} & 5.82622499 & -1.86000751 & -0.00012824 \\ \mathrm{H} & 3.49242299 & -2.70072451 & -0.00013924 \\ \mathrm{C} & 1.65374499 & 1.36543749 & 0.00003976 \\ \mathrm{~N} & 1.28863199 & -0.95749551 & -0.00004924 \\ \mathrm{C} & 0.66075399 & 0.22091049 & 0.00000276 \\ \mathrm{C} & -0.71339001 & 0.24466349 & 0.00001676 \\ \mathrm{H} & 0.33789399 & -1.83936051 & -0.00007624 \\ \mathrm{O} & 1.37283799 & 2.54020249 & 0.00008876 \\ \mathrm{C} & -1.41510601 & -0.99546551 & -0.00002624 \\ \mathrm{~N} & -1.62855701 & 1.28623249 & 0.00006676 \\ \mathrm{H} & -1.38141501 & 2.25977849 & 0.00010376 \\ \mathrm{C} & -2.88982701 & 0.75100449 & 0.00005676 \\ \mathrm{C} & -2.81256201 & -0.66324651 & -0.00000024 \\ \mathrm{O} & -0.81897301 & -2.12791351 & -0.00007724 \\ \mathrm{C} & -4.12930501 & 1.3971549 & 0.00009376 \\ \mathrm{C} & -3.97648501 & -1.43867951 & -0.00002124 \\ \mathrm{C} & -5.26267001 & 0.60776649 & 0.00007276 \\ \mathrm{C} & -5.19709101 & -0.79829951 & 0.00001576 \\ \mathrm{H} & -4.19805301 & 2.47841849 & 0.00013776 \\ \mathrm{H} & -6.23497701 & 1.08786949 & 0.00010076 \\ \mathrm{H} & -6.11454801 & -1.37381351 & 0.00000076 \\ \mathrm{H} & -3.90355701 & -2.51974451 & -0.00006524\end{array}$


$\mathrm{S}_{0}$ Ind (IRC point 20 toward isomer 1), gas phase

$\begin{array}{lrrr}\mathrm{C} & 2.94319000 & 0.66252000 & -0.00000100 \\ \mathrm{C} & 4.24861800 & 1.13720900 & 0.00000500 \\ \mathrm{C} & 5.28705800 & 0.21609000 & -0.00004100 \\ \mathrm{C} & 5.00406000 & -1.15303000 & -0.00009200 \\ \mathrm{C} & 3.70323700 & -1.64159700 & -0.00009900 \\ \mathrm{C} & 2.67046300 & -0.71723300 & -0.00005300 \\ \mathrm{H} & 4.43615600 & 2.20492700 & 0.00004600 \\ \mathrm{H} & 6.31663500 & 0.55182900 & -0.00003800 \\ \mathrm{H} & 5.82601000 & -1.86051500 & -0.00012800 \\ \mathrm{H} & 3.50180600 & -2.70574700 & -0.00013900 \\ \mathrm{C} & 1.64790000 & 1.36112100 & 0.00003900 \\ \mathrm{~N} & 1.31317000 & -0.94835000 & -0.00004900 \\ \mathrm{C} & 0.64697500 & 0.24070800 & 0.00000400 \\ \mathrm{C} & -0.70174000 & 0.25699400 & 0.00001700 \\ \mathrm{H} & 0.76554300 & -1.81046300 & -0.00007900 \\ \mathrm{O} & 1.37294600 & 2.54144900 & 0.00008900 \\ \mathrm{C} & -1.41303400 & -1.02447400 & -0.00002800 \\ \mathrm{~N} & -1.62279000 & 1.28595900 & 0.00006700 \\ \mathrm{H} & -1.37917700 & 2.26075500 & 0.00010400 \\ \mathrm{C} & -2.89349100 & 0.74712100 & 0.00005700 \\ \mathrm{C} & -2.82136600 & -0.66060700 & 0.00000000 \\ \mathrm{O} & -0.85790400 & -2.12523900 & -0.00007700 \\ \mathrm{C} & -4.12617900 & 1.39667400 & 0.00009400 \\ \mathrm{C} & -3.97477100 & -1.43713000 & -0.00002100 \\ \mathrm{C} & -5.26369800 & 0.60467600 & 0.00007300 \\ \mathrm{C} & -5.20028500 & -0.79625400 & 0.00001600 \\ \mathrm{H} & -4.19540600 & 2.47787300 & 0.00013800 \\ \mathrm{H} & -6.23511700 & 1.08675100 & 0.00010100 \\ \mathrm{H} & -6.11724800 & -1.37234100 & 0.00000100 \\ \mathrm{H} & -3.89719900 & -2.51812900 & -0.00006500\end{array}$


$\mathrm{S}_{0}$ Ind (IRC point 40 toward isomer 1), gas phase

$\begin{array}{lrrr}\mathrm{C} & 2.95338500 & 0.67587700 & -0.00000100 \\ \mathrm{C} & 4.25853500 & 1.14822500 & 0.00000600 \\ \mathrm{C} & 5.29706000 & 0.22709800 & -0.00004100 \\ \mathrm{C} & 5.02832000 & -1.14630000 & -0.00009200 \\ \mathrm{C} & 3.73262800 & -1.63785300 & -0.00010000 \\ \mathrm{C} & 2.70564200 & -0.70462500 & -0.00005300 \\ \mathrm{H} & 4.45017700 & 2.21508100 & 0.00004600 \\ \mathrm{H} & 6.32457200 & 0.56898000 & -0.00003700 \\ \mathrm{H} & 5.85672300 & -1.84608500 & -0.00012800 \\ \mathrm{H} & 3.52569700 & -2.70104200 & -0.00014000 \\ \mathrm{C} & 1.64656800 & 1.36231600 & 0.00003900 \\ \mathrm{~N} & 1.35683600 & -0.94539800 & -0.00005000 \\ \mathrm{C} & 0.65464100 & 0.23416200 & 0.00000400 \\ \mathrm{C} & -0.69502100 & 0.23882200 & 0.00001600 \\ \mathrm{H} & 0.87655000 & -1.83701200 & -0.00008100 \\ \mathrm{O} & 1.36882400 & 2.54299600 & 0.00008900 \\ \mathrm{C} & -1.44295000 & -1.04235300 & -0.00002800 \\ \mathrm{~N} & -1.61109800 & 1.27410600 & 0.00006600 \\ \mathrm{H} & -1.35677100 & 2.24658800 & 0.00010300 \\ \mathrm{C} & -2.89487300 & 0.75108500 & 0.00005700 \\ \mathrm{C} & -2.84210400 & -0.65160000 & 0.00000000 \\ \mathrm{O} & -0.94824600 & -2.16198000 & -0.00007700 \\ \mathrm{C} & -4.12553900 & 1.40126500 & 0.00009400 \\ \mathrm{C} & -3.99014400 & -1.43057000 & -0.00002100 \\ \mathrm{C} & -5.26768100 & 0.61036400 & 0.00007300 \\ \mathrm{C} & -5.21558500 & -0.78926100 & 0.00001600 \\ \mathrm{H} & -4.19602700 & 2.48230400 & 0.00013800 \\ \mathrm{H} & -6.23597000 & 1.09872600 & 0.00010100 \\ \mathrm{H} & -6.13533100 & -1.36078300 & 0.00000200 \\ \mathrm{H} & -3.90463100 & -2.51097200 & -0.00006500\end{array}$


$\mathrm{S}_{0}$ Ind (IRC point 60 toward isomer 1), gas phase

$\begin{array}{lrrr}\mathrm{C} & 2.95749600 & 0.67714300 & -0.00000100 \\ \mathrm{C} & 4.25766500 & 1.16799900 & 0.00000700 \\ \mathrm{C} & 5.31093100 & 0.26356600 & -0.00003900 \\ \mathrm{C} & 5.06112000 & -1.11339900 & -0.00009200 \\ \mathrm{C} & 3.77159200 & -1.62251100 & -0.00009900 \\ \mathrm{C} & 2.72735300 & -0.70663000 & -0.00005300 \\ \mathrm{H} & 4.43335300 & 2.23763900 & 0.00004700 \\ \mathrm{H} & 6.33317500 & 0.62097800 & -0.00003500 \\ \mathrm{H} & 5.89838200 & -1.80259400 & -0.00012700 \\ \mathrm{H} & 3.58223500 & -2.68904100 & -0.00014000 \\ \mathrm{C} & 1.64045400 & 1.34609700 & 0.00003900 \\ \mathrm{~N} & 1.37841900 & -0.96632500 & -0.00005000 \\ \mathrm{C} & 0.65853100 & 0.20528400 & 0.00000300 \\ \mathrm{C} & -0.69320500 & 0.20634500 & 0.00001500 \\ \mathrm{H} & 0.91464800 & -1.86549500 & -0.00008200 \\ \mathrm{O} & 1.35130300 & 2.52436500 & 0.00008800 \\ \mathrm{C} & -1.46512100 & -1.06601700 & -0.00002900 \\ \mathrm{~N} & -1.59828500 & 1.25307400 & 0.00006500 \\ \mathrm{H} & -1.32862700 & 2.22168100 & 0.00010200 \\ \mathrm{C} & -2.89155800 & 0.75102700 & 0.00005700 \\ \mathrm{C} & -2.85997400 & -0.65157100 & 0.00000000 \\ \mathrm{O} & -0.99356300 & -2.19385200 & -0.00007800 \\ \mathrm{C} & -4.11436400 & 1.41762900 & 0.00009500 \\ \mathrm{C} & -4.01917800 & -1.41437200 & -0.00002000 \\ \mathrm{C} & -5.26835000 & 0.64275500 & 0.00007400 \\ \mathrm{C} & -5.23664000 & -0.75722400 & 0.00001800 \\ \mathrm{H} & -4.17096700 & 2.49949700 & 0.00013900 \\ \mathrm{H} & -6.22929600 & 1.14546900 & 0.00010300 \\ \mathrm{H} & -6.16398700 & -1.31635500 & 0.00000400 \\ \mathrm{H} & -3.94786600 & -2.49585000 & -0.00006400\end{array}$


$\mathrm{S}_{0}$ Ind (IRC point 80 toward isomer 1), gas phase

$\begin{array}{lrrr}\mathrm{C} & 2.95273100 & 0.67502200 & -0.00000100 \\ \mathrm{C} & 4.24512300 & 1.18602000 & 0.00000700 \\ \mathrm{C} & 5.31218300 & 0.29838100 & -0.00003800 \\ \mathrm{C} & 5.08177200 & -1.08194100 & -0.00009000 \\ \mathrm{C} & 3.79963300 & -1.61067700 & -0.00009900 \\ \mathrm{C} & 2.74011000 & -0.71179600 & -0.00005400 \\ \mathrm{H} & 4.40314100 & 2.25842200 & 0.00004800 \\ \mathrm{H} & 6.32903700 & 0.67086800 & -0.00003300 \\ \mathrm{H} & 5.92877400 & -1.75914400 & -0.00012500 \\ \mathrm{H} & 3.62820800 & -2.68025500 & -0.00014000 \\ \mathrm{C} & 1.62868500 & 1.32716700 & 0.00003800 \\ \mathrm{~N} & 1.39270800 & -0.98825800 & -0.00005100 \\ \mathrm{C} & 0.66048700 & 0.17673700 & 0.00000100 \\ \mathrm{C} & -0.69094300 & 0.17933800 & 0.00001400 \\ \mathrm{H} & 0.94111700 & -1.89289100 & -0.00008400 \\ \mathrm{O} & 1.32589200 & 2.50254500 & 0.00008800 \\ \mathrm{C} & -1.47663600 & -1.08542300 & -0.00002900 \\ \mathrm{~N} & -1.58398900 & 1.23562400 & 0.00006400 \\ \mathrm{H} & -1.30141200 & 2.20078100 & 0.00010100 \\ \mathrm{C} & -2.88278000 & 0.74915800 & 0.00005700 \\ \mathrm{C} & -2.86870900 & -0.65400200 & 0.00000000 \\ \mathrm{O} & -1.01375700 & -2.21599500 & -0.00007900 \\ \mathrm{C} & -4.09572500 & 1.43343400 & 0.00009500 \\ \mathrm{C} & -4.03990500 & -1.39893700 & -0.00001900 \\ \mathrm{C} & -5.26098300 & 0.67585500 & 0.00007500 \\ \mathrm{C} & -5.24829200 & -0.72428100 & 0.00001900 \\ \mathrm{H} & -4.13576000 & 2.51605200 & 0.00013900 \\ \mathrm{H} & -6.21468000 & 1.19219000 & 0.00010500 \\ \mathrm{H} & -6.18317900 & -1.27073300 & 0.00000600 \\ \mathrm{H} & -3.98568500 & -2.48143100 & -0.00006300\end{array}$


$\mathrm{S}_{0}$ Ind (IRC point 100 toward isomer 1), gas phase

$\begin{array}{lrrr}\mathrm{C} & 2.94612700 & 0.67302700 & -0.00000100 \\ \mathrm{C} & 4.23006200 & 1.20410300 & 0.00000800 \\ \mathrm{C} & 5.31061200 & 0.33356100 & -0.00003700 \\ \mathrm{C} & 5.10053100 & -1.04977600 & -0.00008900 \\ \mathrm{C} & 3.82628400 & -1.59853000 & -0.00009900 \\ \mathrm{C} & 2.75197400 & -0.71680400 & -0.00005400 \\ \mathrm{H} & 4.36902400 & 2.27985200 & 0.00004900 \\ \mathrm{H} & 6.32123300 & 0.72418600 & -0.00003100 \\ \mathrm{H} & 5.96023600 & -1.71217600 & -0.00012400 \\ \mathrm{H} & 3.67538800 & -2.67276400 & -0.00014000 \\ \mathrm{C} & 1.61479700 & 1.30715200 & 0.00003700 \\ \mathrm{~N} & 1.40679000 & -1.01112400 & -0.00005300 \\ \mathrm{C} & 0.66233500 & 0.14680700 & 0.00000000 \\ \mathrm{C} & -0.68834500 & 0.15185100 & 0.00001300 \\ \mathrm{H} & 0.96587600 & -1.91821600 & -0.00008400 \\ \mathrm{O} & 1.29602300 & 2.47905800 & 0.00008700 \\ \mathrm{C} & -1.48772000 & -1.10315200 & -0.00003000 \\ \mathrm{~N} & -1.56790000 & 1.21764300 & 0.00006300 \\ \mathrm{H} & -1.26730600 & 2.17697600 & 0.00010000 \\ \mathrm{C} & -2.87215300 & 0.74704100 & 0.00005600 \\ \mathrm{C} & -2.87612700 & -0.65647200 & 0.00000000 \\ \mathrm{O} & -1.03143500 & -2.23836600 & -0.00008000 \\ \mathrm{C} & -4.07404300 & 1.44937000 & 0.00009600 \\ \mathrm{C} & -4.05925000 & -1.38302800 & -0.00001800 \\ \mathrm{C} & -5.25053100 & 0.70991300 & 0.00007700 \\ \mathrm{C} & -5.25770100 & -0.68997800 & 0.00002100 \\ \mathrm{H} & -4.09446300 & 2.53317700 & 0.00013900 \\ \mathrm{H} & -6.19578700 & 1.24290000 & 0.00010700 \\ \mathrm{H} & -6.20261700 & -1.22101200 & 0.00000800 \\ \mathrm{H} & -4.02592100 & -2.46759900 & -0.00006200\end{array}$


$\mathrm{S}_{0}$ Ind (IRC point 120 toward isomer 1), gas phase

$\begin{array}{lrrr}\mathrm{C} & 2.93750100 & 0.67122400 & -0.00000100 \\ \mathrm{C} & 4.21129500 & 1.22615300 & 0.00000900 \\ \mathrm{C} & 5.30730100 & 0.37562600 & -0.00003500 \\ \mathrm{C} & 5.12091600 & -1.01134400 & -0.00008800 \\ \mathrm{C} & 3.85678700 & -1.58345400 & -0.00009800 \\ \mathrm{C} & 2.76578900 & -0.72212200 & -0.00005400 \\ \mathrm{H} & 4.33083500 & 2.30350800 & 0.00005100 \\ \mathrm{H} & 6.31145600 & 0.78106200 & -0.00002800 \\ \mathrm{H} & 5.98943600 & -1.66073900 & -0.00012200 \\ \mathrm{H} & 3.72411600 & -2.65855500 & -0.00014000 \\ \mathrm{C} & 1.59777600 & 1.28345800 & 0.00003600 \\ \mathrm{~N} & 1.42418900 & -1.03767400 & -0.00005400 \\ \mathrm{C} & 0.66491000 & 0.11102900 & -0.00000100 \\ \mathrm{C} & -0.68468200 & 0.11804600 & 0.00001100 \\ \mathrm{H} & 1.00014500 & -1.95410000 & -0.00008600 \\ \mathrm{O} & 1.25876200 & 2.45042400 & 0.00008600 \\ \mathrm{C} & -1.49972800 & -1.12822800 & -0.00003100 \\ \mathrm{~N} & -1.54796300 & 1.19625700 & 0.00006200 \\ \mathrm{H} & -1.23401600 & 2.15235400 & 0.00009800 \\ \mathrm{C} & -2.85793100 & 0.74523800 & 0.00005600 \\ \mathrm{C} & -2.88365000 & -0.65860300 & 0.00000000 \\ \mathrm{O} & -1.05630300 & -2.26511400 & -0.00008000 \\ \mathrm{C} & -4.04688100 & 1.46922200 & 0.00009600 \\ \mathrm{C} & -4.08010300 & -1.36308300 & -0.00001700 \\ \mathrm{C} & -5.23609800 & 0.75078000 & 0.00007800 \\ \mathrm{C} & -5.26649800 & -0.64885500 & 0.00002200 \\ \mathrm{H} & -4.04927200 & 2.55256000 & 0.00014000 \\ \mathrm{H} & -6.17295100 & 1.29706000 & 0.00010900 \\ \mathrm{H} & -6.21806800 & -1.16574400 & 0.00001000 \\ \mathrm{H} & -4.06422700 & -2.44685000 & -0.00006100\end{array}$


$\mathrm{S}_{0}$ Ind (IRC point 140 toward isomer 1), gas phase

$\begin{array}{lrrr}\mathrm{C} & 2.92882800 & 0.66930700 & -0.00000100 \\ \mathrm{C} & 4.19178800 & 1.24802500 & 0.00001000 \\ \mathrm{C} & 5.30299000 & 0.41809000 & -0.00003300 \\ \mathrm{C} & 5.14083500 & -0.97195800 & -0.00008600 \\ \mathrm{C} & 3.88724900 & -1.56753700 & -0.00009800 \\ \mathrm{C} & 2.77977600 & -0.72705500 & -0.00005500 \\ \mathrm{H} & 4.29029900 & 2.32749500 & 0.00005200 \\ \mathrm{H} & 6.29977300 & 0.84131000 & -0.00002600 \\ \mathrm{H} & 6.02079100 & -1.60578500 & -0.00012000 \\ \mathrm{H} & 3.77570800 & -2.64504800 & -0.00014000 \\ \mathrm{C} & 1.58104400 & 1.25932600 & 0.00003600 \\ \mathrm{~N} & 1.44206700 & -1.06411500 & -0.00005500 \\ \mathrm{C} & 0.66754200 & 0.07488200 & -0.00000300 \\ \mathrm{C} & -0.68125100 & 0.08361100 & 0.00001000 \\ \mathrm{H} & 1.03422100 & -1.98710600 & -0.00008800 \\ \mathrm{O} & 1.22270200 & 2.42136400 & 0.00008500 \\ \mathrm{C} & -1.51314500 & -1.15201100 & -0.00003200 \\ \mathrm{~N} & -1.52793600 & 1.17373700 & 0.00006200 \\ \mathrm{H} & -1.19669500 & 2.12441200 & 0.00009600 \\ \mathrm{C} & -2.84365100 & 0.74285000 & 0.00005600 \\ \mathrm{C} & -2.89157100 & -0.66074000 & 0.00000000 \\ \mathrm{O} & -1.08236100 & -2.29294400 & -0.00008100 \\ \mathrm{C} & -4.01869600 & 1.48847200 & 0.00009700 \\ \mathrm{C} & -4.10111800 & -1.34276600 & -0.00001600 \\ \mathrm{C} & -5.22046100 & 0.79181300 & 0.00008000 \\ \mathrm{C} & -5.27474000 & -0.60698900 & 0.00002400 \\ \mathrm{H} & -4.00036400 & 2.57164500 & 0.00014000 \\ \mathrm{H} & -6.14765200 & 1.35432400 & 0.00011100 \\ \mathrm{H} & -6.23517800 & -1.10721900 & 0.00001300 \\ \mathrm{H} & -4.10617600 & -2.42665000 & -0.00006000\end{array}$


$\mathrm{S}_{0}$ Ind (IRC point 160 toward isomer 1), gas phase

$\begin{array}{lrrr}\mathrm{C} & 2.92024900 & 0.66725400 & -0.00000100 \\ \mathrm{C} & 4.17205800 & 1.26920500 & 0.00001200 \\ \mathrm{C} & 5.29782100 & 0.45979100 & -0.00003100 \\ \mathrm{C} & 5.15948400 & -0.93286700 & -0.00008500 \\ \mathrm{C} & 3.91672200 & -1.55135000 & -0.00009800 \\ \mathrm{C} & 2.79346200 & -0.73162800 & -0.00005500 \\ \mathrm{H} & 4.24967000 & 2.35032700 & 0.00005400 \\ \mathrm{H} & 6.28697300 & 0.90046400 & -0.00002300 \\ \mathrm{H} & 6.05049900 & -1.55107300 & -0.00011800 \\ \mathrm{H} & 3.82622400 & -2.63086600 & -0.00014000 \\ \mathrm{C} & 1.56493600 & 1.23535600 & 0.00003500 \\ \mathrm{~N} & 1.46002700 & -1.09012700 & -0.00005700 \\ \mathrm{C} & 0.67031000 & 0.03911300 & -0.00000400 \\ \mathrm{C} & -0.67782000 & 0.04933500 & 0.00000900 \\ \mathrm{H} & 1.06846700 & -2.01953300 & -0.00009000 \\ \mathrm{O} & 1.18825100 & 2.39245600 & 0.00008400 \\ \mathrm{C} & -1.52688100 & -1.17544000 & -0.00003300 \\ \mathrm{~N} & -1.50794400 & 1.15110400 & 0.00006000 \\ \mathrm{H} & -1.15890700 & 2.09592400 & 0.00009400 \\ \mathrm{C} & -2.82921300 & 0.74055000 & 0.00005600 \\ \mathrm{C} & -2.89925200 & -0.66241300 & 0.00000000 \\ \mathrm{O} & -1.11037800 & -2.32101800 & -0.00008200 \\ \mathrm{C} & -3.99012000 & 1.50734000 & 0.00009700 \\ \mathrm{C} & -4.12136100 & -1.32202900 & -0.00001500 \\ \mathrm{C} & -5.20394000 & 0.83241000 & 0.00008100 \\ \mathrm{C} & -5.28192900 & -0.56514000 & 0.00002600 \\ \mathrm{H} & -3.95120400 & 2.58991300 & 0.00014000 \\ \mathrm{H} & -6.12115300 & 1.41104200 & 0.00011300 \\ \mathrm{H} & -6.25098600 & -1.04850500 & 0.00001500 \\ \mathrm{H} & -4.14733300 & -2.40565300 & -0.00005800\end{array}$




$\begin{array}{lrrr}\mathrm{C} & 2.91587400 & 0.66605400 & 0.00000000 \\ \mathrm{C} & 4.16153900 & 1.28050700 & 0.00001200 \\ \mathrm{C} & 5.29491400 & 0.48218400 & -0.00003000 \\ \mathrm{C} & 5.16955100 & -0.91175400 & -0.00008400 \\ \mathrm{C} & 3.93281900 & -1.54246700 & -0.00009800 \\ \mathrm{C} & 2.80135600 & -0.73391200 & -0.00005500 \\ \mathrm{H} & 4.22822600 & 2.36238000 & 0.00005500 \\ \mathrm{H} & 6.27997800 & 0.93201400 & -0.00002100 \\ \mathrm{H} & 6.06633200 & -1.52157400 & -0.00011700 \\ \mathrm{H} & 3.85316900 & -2.62281200 & -0.00014000 \\ \mathrm{C} & 1.55648200 & 1.22212400 & 0.00003400 \\ \mathrm{~N} & 1.47034800 & -1.10342900 & -0.00005800 \\ \mathrm{C} & 0.67189300 & 0.01978600 & -0.00000500 \\ \mathrm{C} & -0.67622200 & 0.03014900 & 0.00000800 \\ \mathrm{H} & 1.08818800 & -2.03636000 & -0.00009100 \\ \mathrm{O} & 1.17041600 & 2.37655800 & 0.00008400 \\ \mathrm{C} & -1.53511000 & -1.18826300 & -0.00003300 \\ \mathrm{~N} & -1.49728200 & 1.13810500 & 0.00006000 \\ \mathrm{H} & -1.13856500 & 2.07954800 & 0.00009400 \\ \mathrm{C} & -2.82147600 & 0.73910400 & 0.00005600 \\ \mathrm{C} & -2.90401300 & -0.66319100 & 0.00000000 \\ \mathrm{O} & -1.12651900 & -2.33610500 & -0.00008300 \\ \mathrm{C} & -3.97439700 & 1.51755700 & 0.00009700 \\ \mathrm{C} & -4.13273200 & -1.31038600 & -0.00001400 \\ \mathrm{C} & -5.19470800 & 0.85475300 & 0.00008200 \\ \mathrm{C} & -5.28590500 & -0.54193700 & 0.00002700 \\ \mathrm{H} & -3.92435300 & 2.59970800 & 0.00014000 \\ \mathrm{H} & -6.10644700 & 1.44198900 & 0.00011400 \\ \mathrm{H} & -6.25946200 & -1.01614900 & 0.00001700 \\ \mathrm{H} & -4.16974000 & -2.39367100 & -0.00005800\end{array}$


$\mathrm{S}_{1}$ Ind (IRC point 1 toward isomer 2), gas phase

$\begin{array}{lrrr}\mathrm{C} & 2.92010894 & 0.68838609 & -0.00001656 \\ \mathrm{C} & 4.23059494 & 1.12478409 & -0.00002056 \\ \mathrm{C} & 5.24636894 & 0.17190609 & -0.00003856 \\ \mathrm{C} & 4.96439094 & -1.2116591 & -0.00005156 \\ \mathrm{C} & 3.66744994 & -1.67517791 & -0.00004756 \\ \mathrm{C} & 2.63367294 & -0.71745491 & -0.00002956 \\ \mathrm{H} & 4.45161794 & 2.18556409 & -0.00001056 \\ \mathrm{H} & 6.28126094 & 0.49406509 & -0.00004256 \\ \mathrm{H} & 5.78650494 & -1.91667991 & -0.00006556 \\ \mathrm{H} & 3.43705794 & -2.73355991 & -0.00005756 \\ \mathrm{C} & 1.63022894 & 1.40952709 & 0.00000044 \\ \mathrm{~N} & 1.30916494 & -0.91956291 & -0.00002256 \\ \mathrm{C} & 0.67359894 & 0.32810709 & -0.00000456 \\ \mathrm{C} & -0.70008106 & 0.26644209 & 0.00000544 \\ \mathrm{H} & 0.41806794 & -1.78611091 & -0.00002456 \\ \mathrm{O} & 1.40020994 & 2.62113109 & 0.00001544 \\ \mathrm{C} & -1.39026206 & -0.99940991 & -0.00000056 \\ \mathrm{~N} & -1.62380206 & 1.28282209 & 0.00002444 \\ \mathrm{H} & -1.37330306 & 2.25758809 & 0.00003444 \\ \mathrm{C} & -2.88983706 & 0.73733709 & 0.00003144 \\ \mathrm{C} & -2.79177406 & -0.67463991 & 0.00001644 \\ \mathrm{O} & -0.80194606 & -2.13058991 & -0.00001756 \\ \mathrm{C} & -4.12840206 & 1.36996009 & 0.00004944 \\ \mathrm{C} & -3.94475506 & -1.46259591 & 0.00001844 \\ \mathrm{C} & -5.25899006 & 0.56657709 & 0.00005144 \\ \mathrm{C} & -5.17598606 & -0.83433391 & 0.00003644 \\ \mathrm{H} & -4.20806606 & 2.45053509 & 0.00006044 \\ \mathrm{H} & -6.23587306 & 1.03702709 & 0.00006544 \\ \mathrm{H} & -6.08585006 & -1.42202291 & 0.00003944 \\ \mathrm{H} & -3.85977006 & -2.54282991 & 0.00000744\end{array}$


$\mathrm{S}_{1}$ Ind (IRC point 20 toward isomer 2), gas phase

$\begin{array}{rrrr}\mathrm{C} & 2.92793700 & 0.70507900 & -0.00001600 \\ \mathrm{C} & 4.24077800 & 1.13456300 & -0.00002100 \\ \mathrm{C} & 5.24721200 & 0.17575200 & -0.00003800 \\ \mathrm{C} & 4.96296100 & -1.21229300 & -0.00005200 \\ \mathrm{C} & 3.66875200 & -1.66873900 & -0.00004700 \\ \mathrm{C} & 2.63820900 & -0.69994600 & -0.00003000 \\ \mathrm{H} & 4.47132500 & 2.19323700 & -0.00001100 \\ \mathrm{H} & 6.28385100 & 0.49286800 & -0.00004200 \\ \mathrm{H} & 5.78616200 & -1.91676300 & -0.00006500 \\ \mathrm{H} & 3.42568400 & -2.72443400 & -0.00005700 \\ \mathrm{C} & 1.63656900 & 1.41193900 & 0.00000000 \\ \mathrm{~N} & 1.32906100 & -0.92319000 & -0.00002300 \\ \mathrm{C} & 0.69069400 & 0.31077000 & -0.00000500 \\ \mathrm{C} & -0.69790000 & 0.25625100 & 0.00000600 \\ \mathrm{H} & 0.15203800 & -1.99171200 & -0.00002400 \\ \mathrm{O} & 1.39424900 & 2.62397300 & 0.00001600 \\ \mathrm{C} & -1.39755100 & -0.97722400 & 0.00000000 \\ \mathrm{~N} & -1.62377100 & 1.28231800 & 0.00002500 \\ \mathrm{H} & -1.36828800 & 2.25580400 & 0.00003400 \\ \mathrm{C} & -2.88436900 & 0.74232900 & 0.00003100 \\ \mathrm{C} & -2.78596500 & -0.67246200 & 0.00001600 \\ \mathrm{O} & -0.84250600 & -2.16111200 & -0.00001700 \\ \mathrm{C} & -4.13004600 & 1.37197700 & 0.00004900 \\ \mathrm{C} & -3.94470100 & -1.46414500 & 0.00001900 \\ \mathrm{C} & -5.25546400 & 0.56915200 & 0.00005200 \\ \mathrm{C} & -5.17111500 & -0.83684500 & 0.00003600 \\ \mathrm{H} & -4.21126300 & 2.45232800 & 0.00006100 \\ \mathrm{H} & -6.23343400 & 1.03733600 & 0.00006500 \\ \mathrm{H} & -6.08161000 & -1.42354700 & 0.00003900 \\ \mathrm{H} & -3.86190000 & -2.54435600 & 0.00000700 \\ & & & \end{array}$


$\mathrm{S}_{1}$ Ind (IRC point 40 toward isomer 2), gas phase

$\begin{array}{lrrr}\mathrm{C} & 2.93680000 & 0.71991900 & -0.00001600 \\ \mathrm{C} & 4.25164100 & 1.15329100 & -0.00002100 \\ \mathrm{C} & 5.26145800 & 0.19623000 & -0.00003800 \\ \mathrm{C} & 4.98340000 & -1.19234600 & -0.00005200 \\ \mathrm{C} & 3.68981600 & -1.65034100 & -0.00004800 \\ \mathrm{C} & 2.65583600 & -0.68858800 & -0.00003000 \\ \mathrm{H} & 4.48317000 & 2.21183500 & -0.00001100 \\ \mathrm{H} & 6.29706100 & 0.51707100 & -0.00004200 \\ \mathrm{H} & 5.80833400 & -1.89472900 & -0.00006500 \\ \mathrm{H} & 3.44681500 & -2.70607200 & -0.00005700 \\ \mathrm{C} & 1.63263000 & 1.40610600 & 0.00000100 \\ \mathrm{~N} & 1.34651700 & -0.93786000 & -0.00002300 \\ \mathrm{C} & 0.70129400 & 0.28504400 & -0.00000500 \\ \mathrm{C} & -0.69205300 & 0.22646900 & 0.00000600 \\ \mathrm{H} & 0.06467900 & -2.09633200 & -0.00002500 \\ \mathrm{O} & 1.36381700 & 2.61333900 & 0.00001600 \\ \mathrm{C} & -1.41772300 & -0.98946800 & 0.00000000 \\ \mathrm{~N} & -1.60312400 & 1.26891900 & 0.00002500 \\ \mathrm{H} & -1.32512100 & 2.23679200 & 0.00003300 \\ \mathrm{C} & -2.87454100 & 0.75328600 & 0.00003100 \\ \mathrm{C} & -2.79383200 & -0.66085400 & 0.00001600 \\ \mathrm{O} & -0.92319900 & -2.20649300 & -0.00001700 \\ \mathrm{C} & -4.11974000 & 1.38622400 & 0.00004900 \\ \mathrm{C} & -3.94946300 & -1.45374900 & 0.00001900 \\ \mathrm{C} & -5.24683500 & 0.58382000 & 0.00005200 \\ \mathrm{C} & -5.17214200 & -0.82331600 & 0.00003700 \\ \mathrm{H} & -4.20252600 & 2.46639100 & 0.00006100 \\ \mathrm{H} & -6.22297400 & 1.05587700 & 0.00006600 \\ \mathrm{H} & -6.08616200 & -1.40443600 & 0.00003900 \\ \mathrm{H} & -3.86367900 & -2.53364200 & 0.00000700\end{array}$


$S_{1}$ Ind (IRC point 60 toward isomer 2), gas phase

$\begin{array}{rrrr}\mathrm{C} & 2.93079200 & 0.71973500 & -0.00001600 \\ \mathrm{C} & 4.23809200 & 1.17605700 & -0.00002000 \\ \mathrm{C} & 5.26451500 & 0.23714500 & -0.00003800 \\ \mathrm{C} & 5.00905900 & -1.15563200 & -0.00005200 \\ \mathrm{C} & 3.72302700 & -1.63591800 & -0.00004800 \\ \mathrm{C} & 2.67052000 & -0.69401500 & -0.00003000 \\ \mathrm{H} & 4.45057900 & 2.23859800 & -0.00001000 \\ \mathrm{H} & 6.29455100 & 0.57546800 & -0.00004100 \\ \mathrm{H} & 5.84536500 & -1.84445300 & -0.00006500 \\ \mathrm{H} & 3.49973000 & -2.69600000 & -0.00005800 \\ \mathrm{C} & 1.61664500 & 1.38356900 & 0.00000100 \\ \mathrm{~N} & 1.36340000 & -0.96598200 & -0.00002400 \\ \mathrm{C} & 0.70457300 & 0.24826100 & -0.00000500 \\ \mathrm{C} & -0.68855300 & 0.19313700 & 0.00000600 \\ \mathrm{H} & 0.03471500 & -2.14440800 & -0.00002500 \\ \mathrm{O} & 1.32233400 & 2.58560100 & 0.00001600 \\ \mathrm{C} & -1.43055300 & -1.01077900 & 0.00000000 \\ \mathrm{~N} & -1.58263300 & 1.24955400 & 0.00002500 \\ \mathrm{H} & -1.28526100 & 2.21220300 & 0.00003300 \\ \mathrm{C} & -2.86089600 & 0.75413500 & 0.00003100 \\ \mathrm{C} & -2.80177500 & -0.66175700 & 0.00001600 \\ \mathrm{O} & -0.95142500 & -2.23601300 & -0.00001800 \\ \mathrm{C} & -4.09574900 & 1.40741700 & 0.00005000 \\ \mathrm{C} & -3.97095200 & -1.43523300 & 0.00001900 \\ \mathrm{C} & -5.23541200 & 0.62359700 & 0.00005200 \\ \mathrm{C} & -5.18301400 & -0.78481300 & 0.00003700 \\ \mathrm{H} & -4.16042300 & 2.48880900 & 0.00006100 \\ \mathrm{H} & -6.20394100 & 1.11107300 & 0.00006600 \\ \mathrm{H} & -6.10626700 & -1.35116400 & 0.00004000 \\ \mathrm{H} & -3.90360100 & -2.51643600 & 0.00000700\end{array}$


$\mathrm{S}_{1}$ Ind (IRC point 80 toward isomer 2), gas phase

$\begin{array}{lrrr}\mathrm{C} & 2.92240800 & 0.71825500 & -0.00001600 \\ \mathrm{C} & 4.22072200 & 1.19883300 & -0.00002000 \\ \mathrm{C} & 5.26374000 & 0.27901300 & -0.00003800 \\ \mathrm{C} & 5.03186800 & -1.11789700 & -0.00005200 \\ \mathrm{C} & 3.75445900 & -1.62183000 & -0.00004800 \\ \mathrm{C} & 2.68359700 & -0.70035500 & -0.00003000 \\ \mathrm{H} & 4.41275500 & 2.26525100 & -0.00000900 \\ \mathrm{H} & 6.28773700 & 0.63517700 & -0.00004100 \\ \mathrm{H} & 5.88005600 & -1.79204900 & -0.00006500 \\ \mathrm{H} & 3.55185700 & -2.68605800 & -0.00005900 \\ \mathrm{C} & 1.59965700 & 1.36019300 & 0.00000100 \\ \mathrm{~N} & 1.37955500 & -0.99402900 & -0.00002400 \\ \mathrm{C} & 0.70684900 & 0.21170900 & -0.00000600 \\ \mathrm{C} & -0.68524400 & 0.15977400 & 0.00000600 \\ \mathrm{H} & 0.01178800 & -2.18623200 & -0.00002600 \\ \mathrm{O} & 1.28206900 & 2.55703400 & 0.00001700 \\ \mathrm{C} & -1.44172200 & -1.03313000 & -0.00000100 \\ \mathrm{~N} & -1.56225500 & 1.22914600 & 0.00002500 \\ \mathrm{H} & -1.24574500 & 2.18628100 & 0.00003300 \\ \mathrm{C} & -2.84620700 & 0.75296600 & 0.00003100 \\ \mathrm{C} & -2.80863400 & -0.66463200 & 0.00001600 \\ \mathrm{O} & -0.97297500 & -2.26403300 & -0.00001800 \\ \mathrm{C} & -4.06900600 & 1.42846100 & 0.00005000 \\ \mathrm{C} & -3.99284800 & -1.41608500 & 0.00001900 \\ \mathrm{C} & -5.22201100 & 0.66555300 & 0.00005200 \\ \mathrm{C} & -5.19304200 & -0.74380900 & 0.00003700 \\ \mathrm{H} & -4.11300800 & 2.51087900 & 0.00006200 \\ \mathrm{H} & -6.18208000 & 1.16946200 & 0.00006600 \\ \mathrm{H} & -6.12590500 & -1.29421400 & 0.00004000 \\ \mathrm{H} & -3.94656800 & -2.49840600 & 0.00000700\end{array}$


$\mathrm{S}_{1}$ Ind (IRC point 100 toward isomer 2), gas phase

$\begin{array}{lrrr}\mathrm{C} & 2.91359000 & 0.71688300 & -0.00001600 \\ \mathrm{C} & 4.20250400 & 1.22148100 & -0.00002000 \\ \mathrm{C} & 5.26177700 & 0.32098900 & -0.00003700 \\ \mathrm{C} & 5.05352600 & -1.07965500 & -0.00005200 \\ \mathrm{C} & 3.78515300 & -1.60696300 & -0.00004900 \\ \mathrm{C} & 2.69646800 & -0.70616200 & -0.00003100 \\ \mathrm{H} & 4.37406000 & 2.29137500 & -0.00000900 \\ \mathrm{H} & 6.27941700 & 0.69489700 & -0.00004100 \\ \mathrm{H} & 5.91330500 & -1.73897500 & -0.00006500 \\ \mathrm{H} & 3.60318700 & -2.67490900 & -0.00005900 \\ \mathrm{C} & 1.58260800 & 1.33676800 & 0.00000100 \\ \mathrm{~N} & 1.39594300 & -1.02164100 & -0.00002500 \\ \mathrm{C} & 0.70905900 & 0.17522900 & -0.00000600 \\ \mathrm{C} & -0.68205300 & 0.12614900 & 0.00000500 \\ \mathrm{H} & -0.01077700 & -2.22654600 & -0.00002600 \\ \mathrm{O} & 1.24238800 & 2.52810700 & 0.00001700 \\ \mathrm{C} & -1.45300400 & -1.05567300 & -0.00000100 \\ \mathrm{~N} & -1.54197700 & 1.20810700 & 0.00002500 \\ \mathrm{H} & -1.20731900 & 2.15966300 & 0.00003300 \\ \mathrm{C} & -2.83123000 & 0.75126300 & 0.00003100 \\ \mathrm{C} & -2.81533100 & -0.66771900 & 0.00001600 \\ \mathrm{O} & -0.99436500 & -2.29196300 & -0.00001800 \\ \mathrm{C} & -4.04145300 & 1.44896200 & 0.00005000 \\ \mathrm{C} & -4.01425700 & -1.39670500 & 0.00001900 \\ \mathrm{C} & -5.20747000 & 0.70732000 & 0.00005300 \\ \mathrm{C} & -5.20206500 & -0.70262000 & 0.00003700 \\ \mathrm{H} & -4.06458000 & 2.53201700 & 0.00006200 \\ \mathrm{H} & -6.15875700 & 1.22758900 & 0.00006700 \\ \mathrm{H} & -6.14433800 & -1.23678100 & 0.00004000 \\ \mathrm{H} & -3.98924700 & -2.47973500 & 0.00000700\end{array}$


$\mathrm{S}_{1}$ Ind (IRC point 120 toward isomer 2), gas phase

$\begin{array}{lrrr}\mathrm{C} & 2.90486400 & 0.71601000 & -0.00001600 \\ \mathrm{C} & 4.18404000 & 1.24426500 & -0.00001900 \\ \mathrm{C} & 5.25905000 & 0.36314300 & -0.00003700 \\ \mathrm{C} & 5.07438500 & -1.04084800 & -0.00005200 \\ \mathrm{C} & 3.81539900 & -1.59111900 & -0.00004900 \\ \mathrm{C} & 2.70960500 & -0.71101000 & -0.00003100 \\ \mathrm{H} & 4.33531000 & 2.31720200 & -0.00000800 \\ \mathrm{H} & 6.27007700 & 0.75455500 & -0.00004000 \\ \mathrm{H} & 5.94543500 & -1.68522000 & -0.00006600 \\ \mathrm{H} & 3.65381100 & -2.66233200 & -0.00006000 \\ \mathrm{C} & 1.56595600 & 1.31361100 & 0.00000100 \\ \mathrm{~N} & 1.41304300 & -1.04820800 & -0.00002500 \\ \mathrm{C} & 0.71151400 & 0.13914200 & -0.00000600 \\ \mathrm{C} & -0.67895500 & 0.09197900 & 0.00000500 \\ \mathrm{H} & -0.03621900 & -2.26964500 & -0.00002700 \\ \mathrm{O} & 1.20408500 & 2.49931200 & 0.00001800 \\ \mathrm{C} & -1.46480900 & -1.07839600 & -0.00000100 \\ \mathrm{~N} & -1.52174900 & 1.18639700 & 0.00002500 \\ \mathrm{H} & -1.16767200 & 2.13177800 & 0.00003300 \\ \mathrm{C} & -2.81612800 & 0.74923400 & 0.00003100 \\ \mathrm{C} & -2.82207300 & -0.67071100 & 0.00001600 \\ \mathrm{O} & -1.01838600 & -2.32077100 & -0.00001900 \\ \mathrm{C} & -4.01355100 & 1.46867100 & 0.00005000 \\ \mathrm{C} & -4.03503900 & -1.37733100 & 0.00001900 \\ \mathrm{C} & -5.19205100 & 0.74826500 & 0.00005300 \\ \mathrm{C} & -5.21015300 & -0.66189200 & 0.00003700 \\ \mathrm{H} & -4.01608500 & 2.55195700 & 0.00006200 \\ \mathrm{H} & -6.13435500 & 1.28461100 & 0.00006700 \\ \mathrm{H} & -6.16151700 & -1.17971400 & 0.00004000 \\ \mathrm{H} & -4.03095500 & -2.46064800 & 0.00000600 \\ & & & \end{array}$




$\begin{array}{lrrr}\mathrm{C} & 2.90177400 & 0.71575200 & -0.00001600 \\ \mathrm{C} & 4.17746800 & 1.25217500 & -0.00001900 \\ \mathrm{C} & 5.25785200 & 0.37782700 & -0.00003700 \\ \mathrm{C} & 5.08144200 & -1.02723900 & -0.00005200 \\ \mathrm{C} & 3.82582300 & -1.58544700 & -0.00004900 \\ \mathrm{C} & 2.71421100 & -0.71257500 & -0.00003100 \\ \mathrm{H} & 4.32171000 & 2.32607600 & -0.00000800 \\ \mathrm{H} & 6.26650900 & 0.77530200 & -0.00004000 \\ \mathrm{H} & 5.95636300 & -1.66634900 & -0.00006600 \\ \mathrm{H} & 3.67129600 & -2.65770000 & -0.00006100 \\ \mathrm{C} & 1.56014800 & 1.30551200 & 0.00000100 \\ \mathrm{~N} & 1.41914100 & -1.05735700 & -0.00002500 \\ \mathrm{C} & 0.71241600 & 0.12655800 & -0.00000600 \\ \mathrm{C} & -0.67786600 & 0.07996200 & 0.00000500 \\ \mathrm{H} & -0.04559800 & -2.28496000 & -0.00002700 \\ \mathrm{O} & 1.19084000 & 2.48917300 & 0.00001800 \\ \mathrm{C} & -1.46903200 & -1.08631600 & -0.00000100 \\ \mathrm{~N} & -1.51466100 & 1.17869100 & 0.00002500 \\ \mathrm{H} & -1.15381100 & 2.12182500 & 0.00003300 \\ \mathrm{C} & -2.81076000 & 0.74851300 & 0.00003200 \\ \mathrm{C} & -2.82438600 & -0.67165400 & 0.00001600 \\ \mathrm{O} & -1.02725900 & -2.33091400 & -0.00001900 \\ \mathrm{C} & -4.00362900 & 1.47548000 & 0.00005100 \\ \mathrm{C} & -4.04208900 & -1.37047600 & 0.00001800 \\ \mathrm{C} & -5.18636400 & 0.76248400 & 0.00005300 \\ \mathrm{C} & -5.21268400 & -0.64766600 & 0.00003700 \\ \mathrm{H} & -3.99902100 & 2.55875500 & 0.00006300 \\ \mathrm{H} & -6.12548300 & 1.30438100 & 0.00006700 \\ \mathrm{H} & -6.16715900 & -1.15973900 & 0.00004000 \\ \mathrm{H} & -4.04523700 & -2.45379900 & 0.00000600\end{array}$


$\mathrm{S}_{1}$ Ind (IRC point 1 toward isomer 1), gas phase

$\begin{array}{lrrr}\mathrm{C} & 2.92010894 & 0.68838609 & -0.00001656 \\ \mathrm{C} & 4.23059494 & 1.12478409 & -0.00002056 \\ \mathrm{C} & 5.24636894 & 0.17190609 & -0.00003856 \\ \mathrm{C} & 4.96439094 & -1.21116591 & -0.00005156 \\ \mathrm{C} & 3.66744994 & -1.67517791 & -0.00004756 \\ \mathrm{C} & 2.63367294 & -0.71745491 & -0.00002956 \\ \mathrm{H} & 4.45161794 & 2.18556409 & -0.00001056 \\ \mathrm{H} & 6.28126094 & 0.49406509 & -0.00004256 \\ \mathrm{H} & 5.78650494 & -1.91667991 & -0.00006556 \\ \mathrm{H} & 3.43705794 & -2.73355991 & -0.00005756 \\ \mathrm{C} & 1.63022894 & 1.40952709 & 0.00000044 \\ \mathrm{~N} & 1.30916494 & -0.91956291 & -0.00002256 \\ \mathrm{C} & 0.67359894 & 0.32810709 & -0.00000456 \\ \mathrm{C} & -0.70008106 & 0.26644209 & 0.00000544 \\ \mathrm{H} & 0.41806794 & -1.78611091 & -0.00002456 \\ \mathrm{O} & 1.40020994 & 2.62113109 & 0.00001544 \\ \mathrm{C} & -1.39026206 & -0.99940991 & -0.00000056 \\ \mathrm{~N} & -1.62380206 & 1.28282209 & 0.00002444 \\ \mathrm{H} & -1.37330306 & 2.25758809 & 0.00003444 \\ \mathrm{C} & -2.88983706 & 0.73733709 & 0.00003144 \\ \mathrm{C} & -2.79177406 & -0.67463991 & 0.00001644 \\ \mathrm{O} & -0.80194606 & -2.13058991 & -0.00001756 \\ \mathrm{C} & -4.12840206 & 1.36996009 & 0.00004944 \\ \mathrm{C} & -3.94475506 & -1.46259591 & 0.00001844 \\ \mathrm{C} & -5.25899006 & 0.56657709 & 0.00005144 \\ \mathrm{C} & -5.17598606 & -0.83433391 & 0.00003644 \\ \mathrm{H} & -4.20806606 & 2.45053509 & 0.00006044 \\ \mathrm{H} & -6.23587306 & 1.03702709 & 0.00006544 \\ \mathrm{H} & -6.08585006 & -1.42202291 & 0.00003944 \\ \mathrm{H} & -3.85977006 & -2.54282991 & 0.00000744\end{array}$


$\mathrm{S}_{1}$ Ind (IRC point 20 toward isomer 1), gas phase

$\begin{array}{lrrr}\mathrm{C} & 2.92690100 & 0.68863500 & -0.00001600 \\ \mathrm{C} & 4.23386500 & 1.12470700 & -0.00002100 \\ \mathrm{C} & 5.25114100 & 0.16913500 & -0.00003900 \\ \mathrm{C} & 4.97377500 & -1.20890700 & -0.00005200 \\ \mathrm{C} & 3.67292500 & -1.67423200 & -0.00004800 \\ \mathrm{C} & 2.66199600 & -0.70910700 & -0.00003000 \\ \mathrm{H} & 4.45531200 & 2.18523500 & -0.00001100 \\ \mathrm{H} & 6.28509900 & 0.49363500 & -0.00004200 \\ \mathrm{H} & 5.79540100 & -1.91459100 & -0.00006500 \\ \mathrm{H} & 3.43889900 & -2.73183200 & -0.00005700 \\ \mathrm{C} & 1.63042600 & 1.40903100 & 0.00000000 \\ \mathrm{~N} & 1.33253800 & -0.88894200 & -0.00002200 \\ \mathrm{C} & 0.66778900 & 0.33310900 & -0.00000500 \\ \mathrm{C} & -0.70080100 & 0.26202700 & 0.00000600 \\ \mathrm{H} & 0.74277800 & -1.74904800 & -0.00002700 \\ \mathrm{O} & 1.40289200 & 2.61846300 & 0.00001500 \\ \mathrm{C} & -1.39740500 & -1.02377100 & -0.00000100 \\ \mathrm{~N} & -1.61837100 & 1.27500600 & 0.00002400 \\ \mathrm{H} & -1.36599200 & 2.25033900 & 0.00003400 \\ \mathrm{C} & -2.89756700 & 0.73522300 & 0.00003100 \\ \mathrm{C} & -2.80410100 & -0.67027500 & 0.00001600 \\ \mathrm{O} & -0.86107600 & -2.14712300 & -0.00001700 \\ \mathrm{C} & -4.12863900 & 1.37132100 & 0.00004900 \\ \mathrm{C} & -3.94575100 & -1.45946500 & 0.00001900 \\ \mathrm{C} & -5.26414300 & 0.56623700 & 0.00005200 \\ \mathrm{C} & -5.18152900 & -0.83003200 & 0.00003700 \\ \mathrm{H} & -4.20847800 & 2.45199900 & 0.00006100 \\ \mathrm{H} & -6.24001100 & 1.03832500 & 0.00006600 \\ \mathrm{H} & -6.09111000 & -1.41842300 & 0.00003900 \\ \mathrm{H} & -3.85408300 & -2.53925500 & 0.00000700\end{array}$


$\mathrm{S}_{1}$ Ind (IRC point 40 toward isomer 1), gas phase

$\begin{array}{lrrr}\mathrm{C} & 2.94557000 & 0.69961000 & -0.00001700 \\ \mathrm{C} & 4.25558900 & 1.13917400 & -0.00002100 \\ \mathrm{C} & 5.27892000 & 0.18835300 & -0.00003900 \\ \mathrm{C} & 5.00857300 & -1.18869600 & -0.00005200 \\ \mathrm{C} & 3.70587500 & -1.65531400 & -0.00004800 \\ \mathrm{C} & 2.69331600 & -0.69608000 & -0.00003000 \\ \mathrm{H} & 4.47623800 & 2.19999800 & -0.00001100 \\ \mathrm{H} & 6.31081100 & 0.51947300 & -0.00004200 \\ \mathrm{H} & 5.83112500 & -1.89341500 & -0.00006500 \\ \mathrm{H} & 3.47407700 & -2.71358200 & -0.00005700 \\ \mathrm{C} & 1.63715300 & 1.40529200 & 0.00000100 \\ \mathrm{~N} & 1.35574400 & -0.89116200 & -0.00002300 \\ \mathrm{C} & 0.67299300 & 0.31385500 & -0.00000500 \\ \mathrm{C} & -0.70213900 & 0.22954000 & 0.00000600 \\ \mathrm{H} & 0.80556000 & -1.76509900 & -0.00002800 \\ \mathrm{O} & 1.39860400 & 2.61152800 & 0.00001600 \\ \mathrm{C} & -1.42655900 & -1.04986300 & -0.00000100 \\ \mathrm{~N} & -1.61237600 & 1.25096000 & 0.00002400 \\ \mathrm{H} & -1.34572400 & 2.22329600 & 0.00003300 \\ \mathrm{C} & -2.90406100 & 0.73441900 & 0.00003100 \\ \mathrm{C} & -2.82916800 & -0.67007400 & 0.00001600 \\ \mathrm{O} & -0.92747700 & -2.18553000 & -0.00001700 \\ \mathrm{C} & -4.12988800 & 1.38297600 & 0.00004900 \\ \mathrm{C} & -3.97633400 & -1.44900000 & 0.00001900 \\ \mathrm{C} & -5.27485200 & 0.58844100 & 0.00005200 \\ \mathrm{C} & -5.20713100 & -0.80819300 & 0.00003700 \\ \mathrm{H} & -4.20131200 & 2.46428000 & 0.00006100 \\ \mathrm{H} & -6.24548500 & 1.07107200 & 0.00006600 \\ \mathrm{H} & -6.12238200 & -1.38781700 & 0.00004000 \\ \mathrm{H} & -3.89206800 & -2.52939900 & 0.00000700\end{array}$


$\mathrm{S}_{1}$ Ind (IRC point 60 toward isomer 1), gas phase

$\begin{array}{lrrr}\mathrm{C} & 2.94542300 & 0.69815100 & -0.00001700 \\ \mathrm{C} & 4.24871200 & 1.16070900 & -0.00002100 \\ \mathrm{C} & 5.28901300 & 0.22906300 & -0.00003800 \\ \mathrm{C} & 5.03958900 & -1.15076600 & -0.00005200 \\ \mathrm{C} & 3.74296000 & -1.63900800 & -0.00004800 \\ \mathrm{C} & 2.71374900 & -0.69945900 & -0.00003000 \\ \mathrm{H} & 4.44965600 & 2.22549800 & -0.00001000 \\ \mathrm{H} & 6.31515600 & 0.57757000 & -0.00004200 \\ \mathrm{H} & 5.87233400 & -1.84352600 & -0.00006500 \\ \mathrm{H} & 3.53142500 & -2.70158200 & -0.00005800 \\ \mathrm{C} & 1.62812400 & 1.38352800 & 0.00000000 \\ \mathrm{~N} & 1.37306700 & -0.91496500 & -0.00002300 \\ \mathrm{C} & 0.67530700 & 0.27781600 & -0.00000500 \\ \mathrm{C} & -0.70164000 & 0.19505800 & 0.00000600 \\ \mathrm{H} & 0.84256100 & -1.79681500 & -0.00002900 \\ \mathrm{O} & 1.37051700 & 2.58584500 & 0.00001600 \\ \mathrm{C} & -1.44355800 & -1.07510200 & -0.00000100 \\ \mathrm{~N} & -1.59853200 & 1.22767400 & 0.00002400 \\ \mathrm{H} & -1.31594000 & 2.19621600 & 0.00003300 \\ \mathrm{C} & -2.89804400 & 0.73138000 & 0.00003100 \\ \mathrm{C} & -2.84442500 & -0.67455200 & 0.00001600 \\ \mathrm{O} & -0.95573400 & -2.21389500 & -0.00001700 \\ \mathrm{C} & -4.11185200 & 1.40260200 & 0.00005000 \\ \mathrm{C} & -4.00713300 & -1.43077800 & 0.00001900 \\ \mathrm{C} & -5.27179500 & 0.63003000 & 0.00005300 \\ \mathrm{C} & -5.22720700 & -0.76733800 & 0.00003800 \\ \mathrm{H} & -4.16226700 & 2.48511400 & 0.00006100 \\ \mathrm{H} & -6.23359400 & 1.12990100 & 0.00006700 \\ \mathrm{H} & -6.15208300 & -1.33157300 & 0.00004000 \\ \mathrm{H} & -3.94488100 & -2.51269100 & 0.00000700\end{array}$


$\mathrm{S}_{1}$ Ind (IRC point 80 toward isomer 1), gas phase

$\begin{array}{rrrr}\mathrm{C} & 2.93975800 & 0.69621200 & -0.00001700 \\ \mathrm{C} & 4.23473900 & 1.18267900 & -0.00002000 \\ \mathrm{C} & 5.29171400 & 0.27073200 & -0.00003800 \\ \mathrm{C} & 5.06493400 & -1.11212300 & -0.00005100 \\ \mathrm{C} & 3.77584600 & -1.62351700 & -0.00004800 \\ \mathrm{C} & 2.73026400 & -0.70406000 & -0.00003100 \\ \mathrm{H} & 4.41525200 & 2.25114600 & -0.00001000 \\ \mathrm{H} & 6.31159600 & 0.63709200 & -0.00004100 \\ \mathrm{H} & 5.90885800 & -1.79130200 & -0.00006500 \\ \mathrm{H} & 3.58496100 & -2.69003700 & -0.00005800 \\ \mathrm{C} & 1.61366100 & 1.35961700 & 0.00000000 \\ \mathrm{~N} & 1.38866400 & -0.94135300 & -0.00002400 \\ \mathrm{C} & 0.67701600 & 0.23953300 & -0.00000500 \\ \mathrm{C} & -0.69970000 & 0.16082800 & 0.00000500 \\ \mathrm{H} & 0.87630000 & -1.83065000 & -0.00002900 \\ \mathrm{O} & 1.33197900 & 2.55690900 & 0.00001600 \\ \mathrm{C} & -1.45683200 & -1.09888600 & -0.00000100 \\ \mathrm{~N} & -1.58056600 & 1.20609800 & 0.00002400 \\ \mathrm{H} & -1.27980100 & 2.17000000 & 0.00003300 \\ \mathrm{C} & -2.88669300 & 0.73008700 & 0.00003100 \\ \mathrm{C} & -2.85424900 & -0.67733700 & 0.00001600 \\ \mathrm{O} & -0.97906800 & -2.24079500 & -0.00001800 \\ \mathrm{C} & -4.08795400 & 1.42325400 & 0.00005000 \\ \mathrm{C} & -4.03083900 & -1.41153200 & 0.00001900 \\ \mathrm{C} & -5.26152300 & 0.67179000 & 0.00005300 \\ \mathrm{C} & -5.23960400 & -0.72599500 & 0.00003800 \\ \mathrm{H} & -4.11788100 & 2.50652700 & 0.00006200 \\ \mathrm{H} & -6.21461600 & 1.18793300 & 0.00006700 \\ \mathrm{H} & -6.17381700 & -1.27469400 & 0.00004100 \\ \mathrm{H} & -3.98957700 & -2.49445300 & 0.00000700\end{array}$


$\mathrm{S}_{1}$ Ind (IRC point 100 toward isomer 1), gas phase

$\begin{array}{rrrr}\mathrm{C} & 2.93320400 & 0.69459700 & -0.00001700 \\ \mathrm{C} & 4.21954100 & 1.20452400 & -0.00002000 \\ \mathrm{C} & 5.29258100 & 0.31226600 & -0.00003800 \\ \mathrm{C} & 5.08843900 & -1.07336400 & -0.00005100 \\ \mathrm{C} & 3.80740500 & -1.60754100 & -0.00004800 \\ \mathrm{C} & 2.74606000 & -0.70808200 & -0.00003100 \\ \mathrm{H} & 4.37990800 & 2.27621800 & -0.00000900 \\ \mathrm{H} & 6.30594600 & 0.69621400 & -0.00004000 \\ \mathrm{H} & 5.94333000 & -1.73876200 & -0.00006500 \\ \mathrm{H} & 3.63681300 & -2.67753300 & -0.00005900 \\ \mathrm{C} & 1.59850700 & 1.33560100 & 0.00000000 \\ \mathrm{~N} & 1.40447600 & -0.96766600 & -0.00002400 \\ \mathrm{C} & 0.67874300 & 0.20126300 & -0.00000500 \\ \mathrm{C} & -0.69755400 & 0.12636100 & 0.00000500 \\ \mathrm{H} & 0.91109100 & -1.86478900 & -0.00003000 \\ \mathrm{O} & 1.29249100 & 2.52743000 & 0.00001600 \\ \mathrm{C} & -1.46981700 & -1.12240400 & -0.00000100 \\ \mathrm{~N} & -1.56204500 & 1.18439900 & 0.00002400 \\ \mathrm{H} & -1.24208000 & 2.14302600 & 0.00003200 \\ \mathrm{C} & -2.87427400 & 0.72894300 & 0.00003100 \\ \mathrm{C} & -2.86314700 & -0.67967800 & 0.00001600 \\ \mathrm{O} & -1.00311600 & -2.26784200 & -0.00001800 \\ \mathrm{C} & -4.06278700 & 1.44358800 & 0.00005000 \\ \mathrm{C} & -4.05282200 & -1.39201400 & 0.00001900 \\ \mathrm{C} & -5.24930000 & 0.71309300 & 0.00005300 \\ \mathrm{C} & -5.24989500 & -0.68480400 & 0.00003800 \\ \mathrm{H} & -4.07252700 & 2.52722500 & 0.00006200 \\ \mathrm{H} & -6.19356400 & 1.24509100 & 0.00006800 \\ \mathrm{H} & -6.19314900 & -1.21787100 & 0.00004100 \\ \mathrm{H} & -4.03212000 & -2.47552200 & 0.00000800\end{array}$


$\mathrm{S}_{1}$ Ind (IRC point 120 toward isomer 1), gas phase

$\begin{array}{lrrr}\mathrm{C} & 2.92617700 & 0.69310800 & -0.00001700 \\ \mathrm{C} & 4.20346400 & 1.22628000 & -0.00002000 \\ \mathrm{C} & 5.29208000 & 0.35387900 & -0.00003700 \\ \mathrm{C} & 5.11063400 & -1.03424500 & -0.00005100 \\ \mathrm{C} & 3.83820600 & -1.59108900 & -0.00004800 \\ \mathrm{C} & 2.76145900 & -0.71174700 & -0.00003100 \\ \mathrm{H} & 4.34371800 & 2.30080700 & -0.00000900 \\ \mathrm{H} & 6.29865900 & 0.75521500 & -0.00004000 \\ \mathrm{H} & 5.97633600 & -1.68559900 & -0.00006500 \\ \mathrm{H} & 3.68785400 & -2.66414100 & -0.00005900 \\ \mathrm{C} & 1.58329600 & 1.31173200 & 0.00000000 \\ \mathrm{~N} & 1.42054400 & -0.99364800 & -0.00002400 \\ \mathrm{C} & 0.68056400 & 0.16333700 & -0.00000600 \\ \mathrm{C} & -0.69532000 & 0.09146300 & 0.00000500 \\ \mathrm{H} & 0.94519000 & -1.89814300 & -0.00003000 \\ \mathrm{O} & 1.25392400 & 2.49787900 & 0.00001600 \\ \mathrm{C} & -1.48288200 & -1.14597800 & -0.00000100 \\ \mathrm{~N} & -1.54360300 & 1.16214000 & 0.00002400 \\ \mathrm{H} & -1.20487600 & 2.11526700 & 0.00003200 \\ \mathrm{C} & -2.86133600 & 0.72751000 & 0.00003100 \\ \mathrm{C} & -2.87168800 & -0.68196200 & 0.00001600 \\ \mathrm{O} & -1.02825500 & -2.29534500 & -0.00001800 \\ \mathrm{C} & -4.03672500 & 1.46353000 & 0.00005000 \\ \mathrm{C} & -4.07400200 & -1.37224900 & 0.00002000 \\ \mathrm{C} & -5.23569100 & 0.75423500 & 0.00005300 \\ \mathrm{C} & -5.25889100 & -0.64348300 & 0.00003800 \\ \mathrm{H} & -4.02622600 & 2.54715100 & 0.00006200 \\ \mathrm{H} & -6.17088900 & 1.30189400 & 0.00006800 \\ \mathrm{H} & -6.21098800 & -1.16064800 & 0.00004200 \\ \mathrm{H} & -4.07385800 & -2.45595000 & 0.00000800\end{array}$


$\mathrm{S}_{1}$ Ind (IRC point 140 toward isomer 1), gas phase

$\begin{array}{lrrr}\mathrm{C} & 2.91884900 & 0.69183900 & -0.00001600 \\ \mathrm{C} & 4.18671400 & 1.24800700 & -0.00001900 \\ \mathrm{C} & 5.29036600 & 0.39569100 & -0.00003700 \\ \mathrm{C} & 5.13170800 & -0.99488700 & -0.00005100 \\ \mathrm{C} & 3.86857100 & -1.57424300 & -0.00004900 \\ \mathrm{C} & 2.77646000 & -0.71502000 & -0.00003100 \\ \mathrm{H} & 4.30677600 & 2.32497300 & -0.00000800 \\ \mathrm{H} & 6.28984900 & 0.81430200 & -0.00003900 \\ \mathrm{H} & 6.00824300 & -1.63171200 & -0.00006500 \\ \mathrm{H} & 3.73842000 & -2.64990700 & -0.00005900 \\ \mathrm{C} & 1.56808300 & 1.28795900 & 0.00000000 \\ \mathrm{~N} & 1.43735300 & -1.01975600 & -0.00002500 \\ \mathrm{C} & 0.68229000 & 0.12601700 & -0.00000600 \\ \mathrm{C} & -0.69312800 & 0.05635200 & 0.00000500 \\ \mathrm{H} & 0.98103600 & -1.93147700 & -0.00003100 \\ \mathrm{O} & 1.21699300 & 2.46847100 & 0.00001600 \\ \mathrm{C} & -1.49626000 & -1.16945200 & -0.00000100 \\ \mathrm{~N} & -1.52550500 & 1.13960500 & 0.00002400 \\ \mathrm{H} & -1.16746600 & 2.08673700 & 0.00003200 \\ \mathrm{C} & -2.84812900 & 0.72598300 & 0.00003100 \\ \mathrm{C} & -2.87999500 & -0.68404500 & 0.00001600 \\ \mathrm{O} & -1.05492900 & -2.32308700 & -0.00001800 \\ \mathrm{C} & -4.01032800 & 1.48292900 & 0.00005000 \\ \mathrm{C} & -4.09438000 & -1.35240400 & 0.00002000 \\ \mathrm{C} & -5.22105400 & 0.79476800 & 0.00005300 \\ \mathrm{C} & -5.26672700 & -0.60253300 & 0.00003800 \\ \mathrm{H} & -3.97971000 & 2.56615900 & 0.00006100 \\ \mathrm{H} & -6.14698200 & 1.35784200 & 0.00006800 \\ \mathrm{H} & -6.22745700 & -1.10352900 & 0.00004200 \\ \mathrm{H} & -4.11454100 & -2.43588100 & 0.00000800\end{array}$




$\begin{array}{lrrr}\mathrm{C} & 2.91566600 & 0.69124300 & -0.00001600 \\ \mathrm{C} & 4.17924700 & 1.25740800 & -0.00001900 \\ \mathrm{C} & 5.28937200 & 0.41381200 & -0.00003700 \\ \mathrm{C} & 5.14060600 & -0.97749000 & -0.00005100 \\ \mathrm{C} & 3.88144700 & -1.56653300 & -0.00004900 \\ \mathrm{C} & 2.78316400 & -0.71614400 & -0.00003100 \\ \mathrm{H} & 4.29071200 & 2.33531000 & -0.00000800 \\ \mathrm{H} & 6.28573600 & 0.83980500 & -0.00003900 \\ \mathrm{H} & 6.02157400 & -1.60819700 & -0.00006500 \\ \mathrm{H} & 3.76002700 & -2.64327300 & -0.00006000 \\ \mathrm{C} & 1.56146700 & 1.27735700 & 0.00000000 \\ \mathrm{~N} & 1.44460300 & -1.03030300 & -0.00002500 \\ \mathrm{C} & 0.68332900 & 0.10925500 & -0.00000600 \\ \mathrm{C} & -0.69213400 & 0.04069000 & 0.00000500 \\ \mathrm{H} & 0.99733700 & -1.94630700 & -0.00003100 \\ \mathrm{O} & 1.20028500 & 2.45512400 & 0.00001600 \\ \mathrm{C} & -1.50225800 & -1.17971000 & -0.00000100 \\ \mathrm{~N} & -1.51716500 & 1.12962900 & 0.00002400 \\ \mathrm{H} & -1.14986200 & 2.07390700 & 0.00003000 \\ \mathrm{C} & -2.84208500 & 0.72553700 & 0.00003100 \\ \mathrm{C} & -2.88346100 & -0.68462400 & 0.00001600 \\ \mathrm{O} & -1.06751300 & -2.33560200 & -0.00001800 \\ \mathrm{C} & -3.99848000 & 1.49143400 & 0.00005000 \\ \mathrm{C} & -4.10281300 & -1.34347600 & 0.00002000 \\ \mathrm{C} & -5.21423600 & 0.81238800 & 0.00005300 \\ \mathrm{C} & -5.26966100 & -0.58455100 & 0.00003900 \\ \mathrm{H} & -3.95945800 & 2.57439000 & 0.00006100 \\ \mathrm{H} & -6.13616300 & 1.38196300 & 0.00006800 \\ \mathrm{H} & -6.23394800 & -1.07870700 & 0.00004200 \\ \mathrm{H} & -4.13156300 & -2.42678900 & 0.00000800\end{array}$


$\mathrm{S}_{0}$ NHxInd (IRC point 1 toward isomer 2), gas phase

$\begin{array}{lrrr}\mathrm{C} & -2.06443612 & -2.35508106 & 0.22383318 \\ \mathrm{C} & -2.75190112 & -3.54718906 & 0.37542018 \\ \mathrm{C} & -4.07508312 & -3.59811706 & -0.04995682 \\ \mathrm{C} & -4.67239112 & -2.46684106 & -0.60886382 \\ \mathrm{C} & -3.98452612 & -1.26460606 & -0.76139582 \\ \mathrm{C} & -2.66598212 & -1.22264606 & -0.33598082 \\ \mathrm{H} & -2.26128212 & -4.40874306 & 0.81408818 \\ \mathrm{H} & -4.64539612 & -4.51326106 & 0.05079618 \\ \mathrm{H} & -5.70519112 & -2.52382006 & -0.93494482 \\ \mathrm{H} & -4.45970712 & -0.39373306 & -1.19568982 \\ \mathrm{C} & -0.68522312 & -1.98513706 & 0.57220418 \\ \mathrm{~N} & -1.78136812 & -0.14440706 & -0.38315182 \\ \mathrm{C} & -0.59966412 & -0.51548006 & 0.12575518 \\ \mathrm{C} & 0.41866788 & 0.43012994 & 0.13283218 \\ \mathrm{H} & -1.64793112 & 1.09860194 & -0.76175282 \\ \mathrm{O} & 0.17584088 & -2.65694006 & 1.08422618 \\ \mathrm{C} & 0.12770488 & 1.72392294 & -0.41282382 \\ \mathrm{~N} & 1.74472988 & 0.42716094 & 0.57611118 \\ \mathrm{C} & 2.29075088 & 1.64984594 & 0.27877218 \\ \mathrm{C} & 1.32678888 & 2.49444994 & -0.31299582 \\ \mathrm{O} & -1.01250912 & 2.06322594 & -0.88708582 \\ \mathrm{C} & 3.59844288 & 2.10931594 & 0.48360418 \\ \mathrm{C} & 1.64390088 & 3.80503794 & -0.68331982 \\ \mathrm{C} & 3.88988388 & 3.40565094 & 0.10732618 \\ \mathrm{C} & 2.92776088 & 4.25665394 & -0.46896282 \\ \mathrm{H} & 4.36217388 & 1.47567794 & 0.91664018 \\ \mathrm{H} & 4.89666188 & 3.77915694 & 0.25895918 \\ \mathrm{H} & 3.20604788 & 5.26584694 & -0.74642882 \\ \mathrm{H} & 0.88600388 & 4.43667794 & -1.13130082 \\ \mathrm{C} & 2.51888088 & -0.71741106 & 1.02779218 \\ \mathrm{C} & 3.11012488 & -1.51848206 & -0.12591482 \\ \mathrm{H} & 1.87106088 & -1.35147906 & 1.62797618 \\ \mathrm{H} & 3.30557588 & -0.33802406 & 1.68384718 \\ \mathrm{H} & 2.31384488 & -1.92388706 & -0.75366182 \\ \mathrm{H} & 3.76038088 & -0.89576906 & -0.74577982 \\ \mathrm{H} & 3.69390988 & -2.35772606 & 0.25923018 \\ & & & \end{array}$


$\mathrm{S}_{0}$ NHxInd (IRC point 20 toward isomer 2), gas phase

$\begin{array}{lrrr}\mathrm{C} & -2.06755000 & -2.37957500 & 0.23136100 \\ \mathrm{C} & -2.75734300 & -3.56694500 & 0.37990900 \\ \mathrm{C} & -4.08262600 & -3.60694700 & -0.04977200 \\ \mathrm{C} & -4.67686000 & -2.47572600 & -0.60753100 \\ \mathrm{C} & -3.98052600 & -1.27663300 & -0.75559800 \\ \mathrm{C} & -2.66688900 & -1.25042200 & -0.32590300 \\ \mathrm{H} & -2.27584900 & -4.43444400 & 0.81688900 \\ \mathrm{H} & -4.65646900 & -4.52022900 & 0.04873300 \\ \mathrm{H} & -5.70929500 & -2.52987800 & -0.93485800 \\ \mathrm{H} & -4.44352500 & -0.39835200 & -1.18822400 \\ \mathrm{C} & -0.68662400 & -1.99530300 & 0.57483900 \\ \mathrm{~N} & -1.79016600 & -0.16232800 & -0.37921500 \\ \mathrm{C} & -0.62299300 & -0.51542400 & 0.11791000 \\ \mathrm{C} & 0.41051600 & 0.44726000 & 0.12329200 \\ \mathrm{H} & -1.61758100 & 1.34887000 & -0.83340600 \\ \mathrm{O} & 0.17891700 & -2.65768400 & 1.08487800 \\ \mathrm{C} & 0.14578600 & 1.72576400 & -0.40852600 \\ \mathrm{~N} & 1.74083900 & 0.42973500 & 0.57137900 \\ \mathrm{C} & 2.29573800 & 1.64742300 & 0.28208100 \\ \mathrm{C} & 1.33274200 & 2.49239700 & -0.31019000 \\ \mathrm{O} & -0.99430800 & 2.13757400 & -0.90570900 \\ \mathrm{C} & 3.60430500 & 2.10929800 & 0.48619400 \\ \mathrm{C} & 1.64674200 & 3.80528600 & -0.68211700 \\ \mathrm{C} & 3.89369300 & 3.40434400 & 0.10899900 \\ \mathrm{C} & 2.92873000 & 4.25519500 & -0.46822500 \\ \mathrm{H} & 4.36961100 & 1.47726000 & 0.91919800 \\ \mathrm{H} & 4.89990700 & 3.77963300 & 0.25935200 \\ \mathrm{H} & 3.20729000 & 5.26441500 & -0.74592600 \\ \mathrm{H} & 0.88824400 & 4.43621300 & -1.12971700 \\ \mathrm{C} & 2.51322000 & -0.71606800 & 1.02579300 \\ \mathrm{C} & 3.10847100 & -1.51814000 & -0.12541000 \\ \mathrm{H} & 1.86688300 & -1.34931400 & 1.62806400 \\ \mathrm{H} & 3.29918400 & -0.33423300 & 1.68119900 \\ \mathrm{H} & 2.31506600 & -1.92523400 & -0.75574600 \\ \mathrm{H} & 3.75899800 & -0.89450000 & -0.74387300 \\ \mathrm{H} & 3.69291900 & -2.35596900 & 0.26197700\end{array}$


$\mathrm{S}_{0}$ NHxInd (IRC point 40 toward isomer 2), gas phase

$\begin{array}{lrrr}\mathrm{C} & -2.06819800 & -2.38747000 & 0.23463900 \\ \mathrm{C} & -2.74432700 & -3.58261500 & 0.38959100 \\ \mathrm{C} & -4.07075700 & -3.64014800 & -0.03448300 \\ \mathrm{C} & -4.67816300 & -2.51684200 & -0.59259100 \\ \mathrm{C} & -3.99424200 & -1.31058400 & -0.74625700 \\ \mathrm{C} & -2.67877600 & -1.26515800 & -0.32345800 \\ \mathrm{H} & -2.24998900 & -4.44195300 & 0.82692700 \\ \mathrm{H} & -4.63246900 & -4.55989500 & 0.06949600 \\ \mathrm{H} & -5.71073300 & -2.58320500 & -0.91548700 \\ \mathrm{H} & -4.47100900 & -0.44114500 & -1.17993000 \\ \mathrm{C} & -0.68888600 & -1.98778300 & 0.56909100 \\ \mathrm{~N} & -1.81163600 & -0.16206500 & -0.38762000 \\ \mathrm{C} & -0.64120300 & -0.50201300 & 0.10371700 \\ \mathrm{C} & 0.39414000 & 0.46772900 & 0.10608000 \\ \mathrm{H} & -1.62063100 & 1.43282400 & -0.86078700 \\ \mathrm{O} & 0.18131700 & -2.64414200 & 1.07700500 \\ \mathrm{C} & 0.14961100 & 1.75137900 & -0.41736500 \\ \mathrm{~N} & 1.72510400 & 0.43516200 & 0.55756800 \\ \mathrm{C} & 2.29590700 & 1.64701700 & 0.27991000 \\ \mathrm{C} & 1.34452700 & 2.50498900 & -0.31132800 \\ \mathrm{O} & -0.98332600 & 2.19486400 & -0.91914100 \\ \mathrm{C} & 3.60918200 & 2.09348700 & 0.49313000 \\ \mathrm{C} & 1.67638800 & 3.81637300 & -0.67495400 \\ \mathrm{C} & 3.91512100 & 3.38614800 & 0.12422000 \\ \mathrm{C} & 2.96196400 & 4.25032300 & -0.45358100 \\ \mathrm{H} & 4.36444400 & 1.44986400 & 0.92559500 \\ \mathrm{H} & 4.92470400 & 3.74821700 & 0.28176800 \\ \mathrm{H} & 3.25459700 & 5.25703500 & -0.72419800 \\ \mathrm{H} & 0.92939900 & 4.45961900 & -1.12216900 \\ \mathrm{C} & 2.48653100 & -0.71432600 & 1.02070200 \\ \mathrm{C} & 3.09376400 & -1.51737900 & -0.12338200 \\ \mathrm{H} & 1.83079300 & -1.34582600 & 1.61257400 \\ \mathrm{H} & 3.26405300 & -0.33677900 & 1.68855100 \\ \mathrm{H} & 2.30673100 & -1.92366800 & -0.76254600 \\ \mathrm{H} & 3.75158500 & -0.89493600 & -0.73490100 \\ \mathrm{H} & 3.67208200 & -2.35585700 & 0.27100800 \\ & & & \end{array}$




$\begin{array}{lrrr}\mathrm{C} & -2.06508300 & -2.38455200 & 0.23318400 \\ \mathrm{C} & -2.72933500 & -3.58570600 & 0.39550000 \\ \mathrm{C} & -4.05716500 & -3.65581600 & -0.02239400 \\ \mathrm{C} & -4.67525100 & -2.53833200 & -0.58116800 \\ \mathrm{C} & -4.00192800 & -1.32611900 & -0.74192900 \\ \mathrm{C} & -2.68468000 & -1.26738900 & -0.32539700 \\ \mathrm{H} & -2.22538500 & -4.43982600 & 0.83362400 \\ \mathrm{H} & -4.61271000 & -4.57929500 & 0.08588500 \\ \mathrm{H} & -5.70905200 & -2.61194800 & -0.90032800 \\ \mathrm{H} & -4.48779900 & -0.46121900 & -1.17652300 \\ \mathrm{C} & -0.68890200 & -1.97705900 & 0.56200000 \\ \mathrm{~N} & -1.82301400 & -0.15792300 & -0.39499000 \\ \mathrm{C} & -0.65062000 & -0.49233500 & 0.09449800 \\ \mathrm{C} & 0.38580100 & 0.47729900 & 0.09771800 \\ \mathrm{H} & -1.62465400 & 1.45379600 & -0.87052300 \\ \mathrm{O} & 0.18529400 & -2.63021600 & 1.06813900 \\ \mathrm{C} & 0.14838600 & 1.76317000 & -0.42316900 \\ \mathrm{~N} & 1.71551000 & 0.43648400 & 0.55163000 \\ \mathrm{C} & 2.29266900 & 1.64525500 & 0.27843400 \\ \mathrm{C} & 1.34758500 & 2.51041400 & -0.31262100 \\ \mathrm{O} & -0.98302400 & 2.21156300 & -0.92562400 \\ \mathrm{C} & 3.60904200 & 2.08044700 & 0.49737500 \\ \mathrm{C} & 1.69142700 & 3.82129800 & -0.66990700 \\ \mathrm{C} & 3.92606200 & 3.37227300 & 0.13461000 \\ \mathrm{C} & 2.98004400 & 4.24497200 & -0.44251200 \\ \mathrm{H} & 4.35888200 & 1.42984000 & 0.92973000 \\ \mathrm{H} & 4.93777300 & 3.72780000 & 0.29563500 \\ \mathrm{H} & 3.27865000 & 5.25137500 & -0.70959100 \\ \mathrm{H} & 0.94973800 & 4.47201200 & -1.11735600 \\ \mathrm{C} & 2.47404400 & -0.71256700 & 1.01986800 \\ \mathrm{C} & 3.07821900 & -1.52218800 & -0.12098500 \\ \mathrm{H} & 1.82083000 & -1.33894400 & 1.61979200 \\ \mathrm{H} & 3.25555100 & -0.33105200 & 1.68058900 \\ \mathrm{H} & 2.29092000 & -1.92777400 & -0.75976600 \\ \mathrm{H} & 3.73934700 & -0.90403900 & -0.73374800 \\ \mathrm{H} & 3.65382400 & -2.36136900 & 0.27663700\end{array}$


$\mathrm{S}_{0}$ NHxInd (IRC point 1 toward isomer 1), gas phase

$\begin{array}{lrrr}\mathrm{C} & -2.06443612 & -2.35508106 & 0.22383318 \\ \mathrm{C} & -2.75190112 & -3.54718906 & 0.37542018 \\ \mathrm{C} & -4.07508312 & -3.59811706 & -0.04995682 \\ \mathrm{C} & -4.67239112 & -2.46684106 & -0.60886382 \\ \mathrm{C} & -3.98452612 & -1.26460606 & -0.76139582 \\ \mathrm{C} & -2.66598212 & -1.22264606 & -0.33598082 \\ \mathrm{H} & -2.26128212 & -4.40874306 & 0.81408818 \\ \mathrm{H} & -4.64539612 & -4.51326106 & 0.05079618 \\ \mathrm{H} & -5.70519112 & -2.52382006 & -0.93494482 \\ \mathrm{H} & -4.45970712 & -0.39373306 & -1.19568982 \\ \mathrm{C} & -0.68522312 & -1.98513706 & 0.57220418 \\ \mathrm{~N} & -1.78136812 & -0.14440706 & -0.38315182 \\ \mathrm{C} & -0.59966412 & -0.51548006 & 0.12575518 \\ \mathrm{C} & 0.41866788 & 0.43012994 & 0.13283218 \\ \mathrm{H} & -1.64793112 & 1.09860194 & -0.76175282 \\ \mathrm{O} & 0.17584088 & -2.65694006 & 1.08422618 \\ \mathrm{C} & 0.12770488 & 1.72392294 & -0.41282382 \\ \mathrm{~N} & 1.74472988 & 0.42716094 & 0.57611118 \\ \mathrm{C} & 2.29075088 & 1.64984594 & 0.27877218 \\ \mathrm{C} & 1.32678888 & 2.49444994 & -0.31299582 \\ \mathrm{O} & -1.01250912 & 2.06322594 & -0.88708582 \\ \mathrm{C} & 3.59844288 & 2.10931594 & 0.48360418 \\ \mathrm{C} & 1.64390088 & 3.80503794 & -0.68331982 \\ \mathrm{C} & 3.88988388 & 3.40565094 & 0.10732618 \\ \mathrm{C} & 2.92776088 & 4.25665394 & -0.46896282 \\ \mathrm{H} & 4.36217388 & 1.47567794 & 0.91664018 \\ \mathrm{H} & 4.89666188 & 3.77915694 & 0.25895918 \\ \mathrm{H} & 3.20604788 & 5.26584694 & -0.74642882 \\ \mathrm{H} & 0.88600388 & 4.43667794 & -1.13130082 \\ \mathrm{C} & 2.51888088 & -0.71741106 & 1.02779218 \\ \mathrm{C} & 3.11012488 & -1.51848206 & -0.12591482 \\ \mathrm{H} & 1.87106088 & -1.35147906 & 1.62797618 \\ \mathrm{H} & 3.30557588 & -0.33802406 & 1.68384718 \\ \mathrm{H} & 2.31384488 & -1.92388706 & -0.75366182 \\ \mathrm{H} & 3.76038088 & -0.89576906 & -0.74577982 \\ \mathrm{H} & 3.69390988 & -2.35772606 & 0.25923018 \\ & & & \end{array}$


$\mathrm{S}_{0}$ NHxInd (IRC point 20 toward isomer 1), gas phase

$\begin{array}{lrrr}\mathrm{C} & -2.06094400 & -2.34796100 & 0.22258100 \\ \mathrm{C} & -2.75238400 & -3.54370500 & 0.37413900 \\ \mathrm{C} & -4.07271200 & -3.59842600 & -0.04906100 \\ \mathrm{C} & -4.67260000 & -2.46528100 & -0.60943100 \\ \mathrm{C} & -3.99139800 & -1.26504100 & -0.76359900 \\ \mathrm{C} & -2.67141400 & -1.22047600 & -0.33881900 \\ \mathrm{H} & -2.25837800 & -4.40295200 & 0.81325700 \\ \mathrm{H} & -4.64293500 & -4.51351200 & 0.05182900 \\ \mathrm{H} & -5.70545300 & -2.52348300 & -0.93506900 \\ \mathrm{H} & -4.47074000 & -0.39664800 & -1.19848900 \\ \mathrm{C} & -0.68298600 & -1.97732400 & 0.57040600 \\ \mathrm{~N} & -1.79499800 & -0.16718500 & -0.37985500 \\ \mathrm{C} & -0.57464300 & -0.52100100 & 0.13556200 \\ \mathrm{C} & 0.41841900 & 0.41183200 & 0.13924500 \\ \mathrm{H} & -1.92021400 & 0.78008800 & -0.74151400 \\ \mathrm{O} & 0.17634600 & -2.65778800 & 1.08484200 \\ \mathrm{C} & 0.10599500 & 1.74313400 & -0.42547300 \\ \mathrm{~N} & 1.73850800 & 0.42277700 & 0.57623100 \\ \mathrm{C} & 2.29091000 & 1.65522300 & 0.27575500 \\ \mathrm{C} & 1.33551400 & 2.49967000 & -0.31215000 \\ \mathrm{O} & -0.98265900 & 2.08826800 & -0.88612000 \\ \mathrm{C} & 3.59543900 & 2.10708300 & 0.48333700 \\ \mathrm{C} & 1.64377800 & 3.80271500 & -0.68262900 \\ \mathrm{C} & 3.88841400 & 3.40872700 & 0.10586000 \\ \mathrm{C} & 2.93192000 & 4.25774300 & -0.46807400 \\ \mathrm{H} & 4.35930700 & 1.47375400 & 0.91617900 \\ \mathrm{H} & 4.89585600 & 3.78011900 & 0.25873600 \\ \mathrm{H} & 3.20941900 & 5.26693200 & -0.74582000 \\ \mathrm{H} & 0.88231300 & 4.43025300 & -1.13088300 \\ \mathrm{C} & 2.51871800 & -0.71814500 & 1.02823100 \\ \mathrm{C} & 3.11029400 & -1.51809500 & -0.12612300 \\ \mathrm{H} & 1.87075200 & -1.35492400 & 1.62566700 \\ \mathrm{H} & 3.30422300 & -0.33632800 & 1.68452600 \\ \mathrm{H} & 2.31263500 & -1.92404300 & -0.75168000 \\ \mathrm{H} & 3.76100400 & -0.89638900 & -0.74680900 \\ \mathrm{H} & 3.69360500 & -2.35731200 & 0.25959400\end{array}$


$\mathrm{S}_{0}$ NHxInd (IRC point 40 toward isomer 1), gas phase

$\begin{array}{lrrr}\mathrm{C} & -2.06051700 & -2.36314000 & 0.22618500 \\ \mathrm{C} & -2.75043900 & -3.55907900 & 0.37879500 \\ \mathrm{C} & -4.07130900 & -3.61825500 & -0.04239900 \\ \mathrm{C} & -4.68558900 & -2.49267700 & -0.60519000 \\ \mathrm{C} & -4.01181200 & -1.29155800 & -0.76199100 \\ \mathrm{C} & -2.68973300 & -1.24893200 & -0.33567600 \\ \mathrm{H} & -2.25650000 & -4.41833600 & 0.81776500 \\ \mathrm{H} & -4.63439600 & -4.53727100 & 0.06262200 \\ \mathrm{H} & -5.71882800 & -2.56144800 & -0.92738400 \\ \mathrm{H} & -4.49110700 & -0.42278900 & -1.19640300 \\ \mathrm{C} & -0.68159200 & -1.97811700 & 0.57063500 \\ \mathrm{~N} & -1.82246800 & -0.19147500 & -0.37855900 \\ \mathrm{C} & -0.58337300 & -0.52253300 & 0.13334400 \\ \mathrm{C} & 0.40255200 & 0.41965100 & 0.13267300 \\ \mathrm{H} & -1.99503100 & 0.73783900 & -0.74702400 \\ \mathrm{O} & 0.17876500 & -2.65773700 & 1.08692000 \\ \mathrm{C} & 0.11413800 & 1.77809600 & -0.43215800 \\ \mathrm{~N} & 1.72367200 & 0.42272700 & 0.56963100 \\ \mathrm{C} & 2.29479700 & 1.65411000 & 0.27433400 \\ \mathrm{C} & 1.35646900 & 2.51097800 & -0.30991600 \\ \mathrm{O} & -0.94592500 & 2.17334600 & -0.89992600 \\ \mathrm{C} & 3.59931600 & 2.10108700 & 0.48392800 \\ \mathrm{C} & 1.66452600 & 3.81191400 & -0.67986100 \\ \mathrm{C} & 3.89964400 & 3.40507800 & 0.11003800 \\ \mathrm{C} & 2.95381000 & 4.26356600 & -0.46365200 \\ \mathrm{H} & 4.36182500 & 1.46571600 & 0.91615800 \\ \mathrm{H} & 4.90939500 & 3.76815500 & 0.26763100 \\ \mathrm{H} & 3.23823700 & 5.27162100 & -0.73830900 \\ \mathrm{H} & 0.90045400 & 4.43675200 & -1.12743500 \\ \mathrm{C} & 2.50411900 & -0.71879100 & 1.02545400 \\ \mathrm{C} & 3.10835900 & -1.51381400 & -0.12604900 \\ \mathrm{H} & 1.85481900 & -1.36036800 & 1.61580500 \\ \mathrm{H} & 3.28271900 & -0.33393100 & 1.68832600 \\ \mathrm{H} & 2.31647300 & -1.92713400 & -0.75411300 \\ \mathrm{H} & 3.75694100 & -0.88793700 & -0.74478900 \\ \mathrm{H} & 3.69656000 & -2.34791000 & 0.26340300\end{array}$


$\mathrm{S}_{0}$ NHxInd (IRC point 60 toward isomer 1), gas phase

$\begin{array}{lrrr}\mathrm{C} & -2.06080300 & -2.36165400 & 0.22379400 \\ \mathrm{C} & -2.72886900 & -3.56897300 & 0.38666400 \\ \mathrm{C} & -4.05062000 & -3.65362000 & -0.02582700 \\ \mathrm{C} & -4.68431100 & -2.53954300 & -0.58984000 \\ \mathrm{C} & -4.03088800 & -1.32729300 & -0.75611200 \\ \mathrm{C} & -2.70608700 & -1.25704100 & -0.33851800 \\ \mathrm{H} & -2.21566400 & -4.41610800 & 0.82713300 \\ \mathrm{H} & -4.59825600 & -4.58107300 & 0.08656500 \\ \mathrm{H} & -5.71812700 & -2.62503000 & -0.90623200 \\ \mathrm{H} & -4.53009600 & -0.47050000 & -1.19197600 \\ \mathrm{C} & -0.68700900 & -1.96094600 & 0.56254700 \\ \mathrm{~N} & -1.84894600 & -0.18716700 & -0.38767900 \\ \mathrm{C} & -0.60184600 & -0.50474400 & 0.12106100 \\ \mathrm{C} & 0.38513100 & 0.43796300 & 0.11988400 \\ \mathrm{H} & -2.03521900 & 0.73771800 & -0.75718900 \\ \mathrm{O} & 0.17614100 & -2.63639600 & 1.08029500 \\ \mathrm{C} & 0.11296200 & 1.80570400 & -0.43967900 \\ \mathrm{~N} & 1.70593200 & 0.42775600 & 0.55808700 \\ \mathrm{C} & 2.29236900 & 1.65333400 & 0.27025700 \\ \mathrm{C} & 1.36708100 & 2.52522600 & -0.31192500 \\ \mathrm{O} & -0.94279800 & 2.21115600 & -0.90464500 \\ \mathrm{C} & 3.60329500 & 2.07823500 & 0.48760300 \\ \mathrm{C} & 1.69951400 & 3.82380300 & -0.67200800 \\ \mathrm{C} & 3.92650900 & 3.37934900 & 0.12376200 \\ \mathrm{C} & 2.99545600 & 4.25466200 & -0.44799400 \\ \mathrm{H} & 4.35351600 & 1.42750700 & 0.91829300 \\ \mathrm{H} & 4.94116800 & 3.72563700 & 0.28751700 \\ \mathrm{H} & 3.29533500 & 5.26013400 & -0.71568800 \\ \mathrm{H} & 0.94865200 & 4.46519000 & -1.11861800 \\ \mathrm{C} & 2.47857500 & -0.71586300 & 1.02095100 \\ \mathrm{C} & 3.09991700 & -1.50513900 & -0.12523700 \\ \mathrm{H} & 1.82170500 & -1.35989800 & 1.59882300 \\ \mathrm{H} & 3.24745800 & -0.33360600 & 1.69661300 \\ \mathrm{H} & 2.31746200 & -1.91872600 & -0.76486600 \\ \mathrm{H} & 3.75454300 & -0.87558900 & -0.73386100 \\ \mathrm{H} & 3.68574700 & -2.33863100 & 0.26908400\end{array}$


$\mathrm{S}_{0}$ NHxInd (IRC point 80 toward isomer 1), gas phase

$\begin{array}{lrrr}\mathrm{C} & -2.05739800 & -2.35471700 & 0.21923500 \\ \mathrm{C} & -2.69835900 & -3.57429400 & 0.39325900 \\ \mathrm{C} & -4.02029900 & -3.68715200 & -0.00930300 \\ \mathrm{C} & -4.67666500 & -2.58651800 & -0.57354200 \\ \mathrm{C} & -4.04813200 & -1.36150300 & -0.75050100 \\ \mathrm{C} & -2.72141800 & -1.26139500 & -0.34311100 \\ \mathrm{H} & -2.16289700 & -4.40701200 & 0.83472300 \\ \mathrm{H} & -4.55021600 & -4.62389800 & 0.11069000 \\ \mathrm{H} & -5.71090000 & -2.69201600 & -0.88255400 \\ \mathrm{H} & -4.56968400 & -0.51842500 & -1.18698800 \\ \mathrm{C} & -0.69098400 & -1.93656000 & 0.55220100 \\ \mathrm{~N} & -1.87638400 & -0.17866900 & -0.39870200 \\ \mathrm{C} & -0.62284500 & -0.48300800 & 0.10782800 \\ \mathrm{C} & 0.36578500 & 0.45726000 & 0.10922300 \\ \mathrm{H} & -2.07464000 & 0.74278200 & -0.76826200 \\ \mathrm{O} & 0.17735600 & -2.60517600 & 1.07101800 \\ \mathrm{C} & 0.10869800 & 1.83165600 & -0.44509800 \\ \mathrm{~N} & 1.68494300 & 0.43145700 & 0.54972400 \\ \mathrm{C} & 2.28598900 & 1.64969400 & 0.26699900 \\ \mathrm{C} & 1.37398900 & 2.53736300 & -0.31250500 \\ \mathrm{O} & -0.94448500 & 2.24382100 & -0.90746200 \\ \mathrm{C} & 3.60410800 & 2.04803100 & 0.48947000 \\ \mathrm{C} & 1.73322900 & 3.83217700 & -0.66273100 \\ \mathrm{C} & 3.95235900 & 3.34443100 & 0.13510400 \\ \mathrm{C} & 3.03683700 & 4.23831000 & -0.43244000 \\ \mathrm{H} & 4.34081500 & 1.38003800 & 0.91664300 \\ \mathrm{H} & 4.97270800 & 3.67170100 & 0.30249700 \\ \mathrm{H} & 3.35413800 & 5.24023900 & -0.69332900 \\ \mathrm{H} & 0.99734500 & 4.49209300 & -1.10733400 \\ \mathrm{C} & 2.45234500 & -0.71313600 & 1.01879600 \\ \mathrm{C} & 3.08545100 & -1.49873600 & -0.12320800 \\ \mathrm{H} & 1.79142600 & -1.35830200 & 1.58887700 \\ \mathrm{H} & 3.21432400 & -0.33109400 & 1.70224200 \\ \mathrm{H} & 2.30955300 & -1.91163700 & -0.77119100 \\ \mathrm{H} & 3.74512900 & -0.86731200 & -0.72446200 \\ \mathrm{H} & 3.66854400 & -2.33248300 & 0.27465400\end{array}$




$\begin{array}{lrrr}\mathrm{C} & -2.05712900 & -2.35435800 & 0.21893100 \\ \mathrm{C} & -2.69672900 & -3.57455300 & 0.39337100 \\ \mathrm{C} & -4.01865300 & -3.68882900 & -0.00873100 \\ \mathrm{C} & -4.67614500 & -2.58884400 & -0.57289500 \\ \mathrm{C} & -4.04887000 & -1.36317900 & -0.75025500 \\ \mathrm{C} & -2.72208800 & -1.26155400 & -0.34333500 \\ \mathrm{H} & -2.16017000 & -4.40655400 & 0.83481100 \\ \mathrm{H} & -4.54764000 & -4.62604500 & 0.11155500 \\ \mathrm{H} & -5.71037000 & -2.69533400 & -0.88157000 \\ \mathrm{H} & -4.57156000 & -0.52079000 & -1.18670300 \\ \mathrm{C} & -0.69112500 & -1.93535300 & 0.55168800 \\ \mathrm{~N} & -1.87774200 & -0.17807800 & -0.39913600 \\ \mathrm{C} & -0.62388500 & -0.48186500 & 0.10731200 \\ \mathrm{C} & 0.36480900 & 0.45822900 & 0.10893000 \\ \mathrm{H} & -2.07672600 & 0.74316500 & -0.76868500 \\ \mathrm{O} & 0.17749300 & -2.60365000 & 1.07048200 \\ \mathrm{C} & 0.10848200 & 1.83295600 & -0.44514200 \\ \mathrm{~N} & 1.68389400 & 0.43167200 & 0.54952500 \\ \mathrm{C} & 2.28568600 & 1.64951600 & 0.26691100 \\ \mathrm{C} & 1.37432600 & 2.53793700 & -0.31241200 \\ \mathrm{O} & -0.94465600 & 2.24566600 & -0.90742400 \\ \mathrm{C} & 3.60418200 & 2.04653300 & 0.48937800 \\ \mathrm{C} & 1.73484300 & 3.83251000 & -0.66231800 \\ \mathrm{C} & 3.95365400 & 3.34264700 & 0.13529200 \\ \mathrm{C} & 3.03885500 & 4.23741600 & -0.43196800 \\ \mathrm{H} & 4.34025300 & 1.37772600 & 0.91631100 \\ \mathrm{H} & 4.97430900 & 3.66893100 & 0.30266800 \\ \mathrm{H} & 3.35701900 & 5.23911500 & -0.69265800 \\ \mathrm{H} & 0.99965400 & 4.49327800 & -1.10677400 \\ \mathrm{C} & 2.45103200 & -0.71296500 & 1.01889700 \\ \mathrm{C} & 3.08417800 & -1.49878300 & -0.12291800 \\ \mathrm{H} & 1.79000400 & -1.35796500 & 1.58896100 \\ \mathrm{H} & 3.21295600 & -0.33086600 & 1.70237600 \\ \mathrm{H} & 2.30829100 & -1.91168300 & -0.77090700 \\ \mathrm{H} & 3.74398500 & -0.86753000 & -0.72421200 \\ \mathrm{H} & 3.66712500 & -2.33254400 & 0.27512400 \\ & & & \end{array}$


$\mathrm{S}_{1}$ NHxInd (IRC point 1 toward isomer 2), gas phase

$\begin{array}{lrrr}\mathrm{C} & -2.02534653 & -2.34796043 & 0.22568407 \\ \mathrm{C} & -2.73184653 & -3.52888343 & 0.35473607 \\ \mathrm{C} & -4.06243153 & -3.54825643 & -0.05469893 \\ \mathrm{C} & -4.68468353 & -2.40200043 & -0.59019593 \\ \mathrm{C} & -3.99817653 & -1.21341443 & -0.72874293 \\ \mathrm{C} & -2.65406853 & -1.19075843 & -0.31559793 \\ \mathrm{H} & -2.25233253 & -4.40896243 & 0.76678707 \\ \mathrm{H} & -4.63829353 & -4.46171643 & 0.03875507 \\ \mathrm{H} & -5.72205953 & -2.46003043 & -0.89752993 \\ \mathrm{H} & -4.46538953 & -0.32692643 & -1.14029493 \\ \mathrm{C} & -0.63324653 & -1.99591543 & 0.55390007 \\ \mathrm{~N} & -1.78470753 & -0.16753443 & -0.34645993 \\ \mathrm{C} & -0.55270653 & -0.58958643 & 0.15882707 \\ \mathrm{C} & 0.41555347 & 0.41065657 & 0.13009507 \\ \mathrm{H} & -1.68201253 & 1.03464457 & -0.70942193 \\ \mathrm{O} & 0.23132447 & -2.73113043 & 1.03635207 \\ \mathrm{C} & 0.09839747 & 1.71900657 & -0.41020493 \\ \mathrm{~N} & 1.73215647 & 0.41664657 & 0.54094607 \\ \mathrm{C} & 2.28001847 & 1.65263557 & 0.25184407 \\ \mathrm{C} & 1.30196447 & 2.49205457 & -0.32032793 \\ \mathrm{O} & -1.04015953 & 2.06784857 & -0.86690393 \\ \mathrm{C} & 3.58025347 & 2.10806957 & 0.45165007 \\ \mathrm{C} & 1.60895547 & 3.80296357 & -0.6855593 \\ \mathrm{C} & 3.86725547 & 3.41439257 & 0.08075207 \\ \mathrm{C} & 2.89829547 & 4.25925657 & -0.47976893 \\ \mathrm{H} & 4.34738347 & 1.47158157 & 0.87479607 \\ \mathrm{H} & 4.87361947 & 3.79038457 & 0.22739607 \\ \mathrm{H} & 3.16742947 & 5.27180457 & -0.75454893 \\ \mathrm{H} & 0.84520947 & 4.43557457 & -1.12208593 \\ \mathrm{C} & 2.49327247 & -0.71820643 & 1.04190807 \\ \mathrm{C} & 3.14529447 & -1.51828143 & -0.07948693 \\ \mathrm{H} & 1.81227847 & -1.36384343 & 1.59538907 \\ \mathrm{H} & 3.24003347 & -0.32723943 & 1.73727307 \\ \mathrm{H} & 2.37722747 & -1.94695543 & -0.72591293 \\ \mathrm{H} & 3.81122447 & -0.89409343 & -0.68126693 \\ \mathrm{H} & 3.72389747 & -2.34290143 & 0.34266207\end{array}$


$\mathrm{S}_{1}$ NHxInd (IRC point 20 toward isomer 2), gas phase

$\begin{array}{lrrr}\mathrm{C} & -2.02059900 & -2.36644500 & 0.23241300 \\ \mathrm{C} & -2.73312200 & -3.54440300 & 0.35886500 \\ \mathrm{C} & -4.06113000 & -3.55262400 & -0.05304800 \\ \mathrm{C} & -4.68495700 & -2.40168700 & -0.59038300 \\ \mathrm{C} & -3.99600000 & -1.22017000 & -0.72538700 \\ \mathrm{C} & -2.64779500 & -1.20586300 & -0.30807500 \\ \mathrm{H} & -2.26248300 & -4.42985400 & 0.76941300 \\ \mathrm{H} & -4.64156600 & -4.46368100 & 0.03777600 \\ \mathrm{H} & -5.72230900 & -2.46113800 & -0.89766200 \\ \mathrm{H} & -4.45254000 & -0.32706000 & -1.13488200 \\ \mathrm{C} & -0.63457100 & -2.00188300 & 0.55534400 \\ \mathrm{~N} & -1.80216700 & -0.17737100 & -0.34806000 \\ \mathrm{C} & -0.57450300 & -0.59025400 & 0.15229300 \\ \mathrm{C} & 0.40571100 & 0.41659000 & 0.12587800 \\ \mathrm{H} & -1.64346400 & 1.33255300 & -0.79572300 \\ \mathrm{O} & 0.23868600 & -2.73036700 & 1.03858900 \\ \mathrm{C} & 0.11616400 & 1.71354100 & -0.40343200 \\ \mathrm{~N} & 1.73140100 & 0.41651200 & 0.54082400 \\ \mathrm{C} & 2.27905100 & 1.64423100 & 0.25471300 \\ \mathrm{C} & 1.30072300 & 2.48568600 & -0.31919600 \\ \mathrm{O} & -1.02608300 & 2.12761300 & -0.88195300 \\ \mathrm{C} & 3.58257700 & 2.10690400 & 0.45291000 \\ \mathrm{C} & 1.60787000 & 3.80304700 & -0.68582700 \\ \mathrm{C} & 3.86533600 & 3.40932700 & 0.08197100 \\ \mathrm{C} & 2.89243400 & 4.25716100 & -0.48061800 \\ \mathrm{H} & 4.35125800 & 1.47221800 & 0.87576100 \\ \mathrm{H} & 4.87108200 & 3.78765900 & 0.22728400 \\ \mathrm{H} & 3.16289500 & 5.26948000 & -0.75484800 \\ \mathrm{H} & 0.84432200 & 4.43598100 & -1.12182400 \\ \mathrm{C} & 2.49323600 & -0.72002000 & 1.04151900 \\ \mathrm{C} & 3.14593900 & -1.51877000 & -0.07936400 \\ \mathrm{H} & 1.81326800 & -1.36524900 & 1.59550200 \\ \mathrm{H} & 3.23904600 & -0.32804800 & 1.73730700 \\ \mathrm{H} & 2.37861300 & -1.94727800 & -0.72673800 \\ \mathrm{H} & 3.81206900 & -0.89419200 & -0.68064600 \\ \mathrm{H} & 3.72461100 & -2.34384800 & 0.34184800\end{array}$


$\mathrm{S}_{1}$ NHxInd (IRC point 40 toward isomer 2), gas phase

$\begin{array}{lrrr}\mathrm{C} & -2.01964100 & -2.37299700 & 0.23538900 \\ \mathrm{C} & -2.72259200 & -3.55830000 & 0.36587300 \\ \mathrm{C} & -4.05178700 & -3.57916600 & -0.04262000 \\ \mathrm{C} & -4.68540700 & -2.43403700 & -0.57952300 \\ \mathrm{C} & -4.00511000 & -1.24710800 & -0.71772300 \\ \mathrm{C} & -2.65630100 & -1.21719500 & -0.30609300 \\ \mathrm{H} & -2.24367100 & -4.43934900 & 0.77645000 \\ \mathrm{H} & -4.62439300 & -4.49495700 & 0.05079700 \\ \mathrm{H} & -5.72302300 & -2.50087500 & -0.88436000 \\ \mathrm{H} & -4.47068400 & -0.35877300 & -1.12755400 \\ \mathrm{C} & -0.63698700 & -1.99228300 & 0.55120600 \\ \mathrm{~N} & -1.82113100 & -0.17448200 & -0.35587300 \\ \mathrm{C} & -0.59350000 & -0.57813500 & 0.14123800 \\ \mathrm{C} & 0.38978800 & 0.43092100 & 0.11453300 \\ \mathrm{H} & -1.64818900 & 1.42224000 & -0.82495000 \\ \mathrm{O} & 0.24663000 & -2.70915400 & 1.03386300 \\ \mathrm{C} & 0.12080900 & 1.73346300 & -0.40847200 \\ \mathrm{~N} & 1.71640200 & 0.41474100 & 0.53275600 \\ \mathrm{C} & 2.27913500 & 1.63713300 & 0.25572000 \\ \mathrm{C} & 1.31157800 & 2.48906800 & -0.31746800 \\ \mathrm{O} & -1.00864500 & 2.18796800 & -0.89365400 \\ \mathrm{C} & 3.58526300 & 2.09120600 & 0.45885900 \\ \mathrm{C} & 1.62412700 & 3.80501600 & -0.68119300 \\ \mathrm{C} & 3.87535300 & 3.39318900 & 0.09110400 \\ \mathrm{C} & 2.91022100 & 4.24996300 & -0.47228800 \\ \mathrm{H} & 4.35030600 & 1.45224600 & 0.88177700 \\ \mathrm{H} & 4.88300300 & 3.76505200 & 0.23979100 \\ \mathrm{H} & 3.18901600 & 5.26087600 & -0.74320900 \\ \mathrm{H} & 0.86360700 & 4.44158900 & -1.11699900 \\ \mathrm{C} & 2.47502100 & -0.72301600 & 1.03762700 \\ \mathrm{C} & 3.14060800 & -1.51762500 & -0.07880500 \\ \mathrm{H} & 1.79334400 & -1.37083700 & 1.58513200 \\ \mathrm{H} & 3.21447000 & -0.32902400 & 1.73910200 \\ \mathrm{H} & 2.38104800 & -1.94967800 & -0.73289000 \\ \mathrm{H} & 3.80881800 & -0.88961300 & -0.67421600 \\ \mathrm{H} & 3.72001700 & -2.33985600 & 0.34702000 \\ & & & \end{array}$




$\begin{array}{lrrr}\mathrm{C} & -2.01656900 & -2.36798200 & 0.23299600 \\ \mathrm{C} & -2.69931900 & -3.56340000 & 0.37389600 \\ \mathrm{C} & -4.02990500 & -3.60579800 & -0.02571900 \\ \mathrm{C} & -4.68096500 & -2.47069100 & -0.56332200 \\ \mathrm{C} & -4.01894900 & -1.27385000 & -0.71149900 \\ \mathrm{C} & -2.66754800 & -1.22035100 & -0.30908300 \\ \mathrm{H} & -2.20254000 & -4.43395300 & 0.78563200 \\ \mathrm{H} & -4.58968500 & -4.52873400 & 0.07486400 \\ \mathrm{H} & -5.71946000 & -2.55266500 & -0.86157400 \\ \mathrm{H} & -4.50219800 & -0.39562400 & -1.12267600 \\ \mathrm{C} & -0.63889600 & -1.97425400 & 0.54075300 \\ \mathrm{~N} & -1.84184700 & -0.16758500 & -0.36626100 \\ \mathrm{C} & -0.60955000 & -0.56129300 & 0.12778000 \\ \mathrm{C} & 0.37584100 & 0.44607100 & 0.10308000 \\ \mathrm{H} & -1.65417500 & 1.45515000 & -0.83686800 \\ \mathrm{O} & 0.25098400 & -2.68537400 & 1.02075200 \\ \mathrm{C} & 0.11895600 & 1.75195800 & -0.41554500 \\ \mathrm{~N} & 1.70110200 & 0.41725700 & 0.52425900 \\ \mathrm{C} & 2.27446900 & 1.63452200 & 0.25324600 \\ \mathrm{C} & 1.31674500 & 2.49827500 & -0.31940800 \\ \mathrm{O} & -1.00915200 & 2.21346700 & -0.90059800 \\ \mathrm{C} & 3.58570000 & 2.06991200 & 0.46398700 \\ \mathrm{C} & 1.64968100 & 3.81248900 & -0.67422800 \\ \mathrm{C} & 3.89421000 & 3.36932100 & 0.10501900 \\ \mathrm{C} & 2.94076700 & 4.23987100 & -0.45730000 \\ \mathrm{H} & 4.34029400 & 1.41811600 & 0.88581300 \\ \mathrm{H} & 4.90573500 & 3.72811500 & 0.25931200 \\ \mathrm{H} & 3.23243700 & 5.24888300 & -0.72179000 \\ \mathrm{H} & 0.90067600 & 4.46276700 & -1.10978700 \\ \mathrm{C} & 2.45480400 & -0.72046600 & 1.03643100 \\ \mathrm{C} & 3.12429300 & -1.51889900 & -0.07470500 \\ \mathrm{H} & 1.77104600 & -1.36544000 & 1.58335000 \\ \mathrm{H} & 3.19183000 & -0.32444500 & 1.73921300 \\ \mathrm{H} & 2.36712800 & -1.95217900 & -0.73062900 \\ \mathrm{H} & 3.79590600 & -0.89342100 & -0.66901600 \\ \mathrm{H} & 3.70089100 & -2.34048900 & 0.35615800 \\ & & & \end{array}$


$\mathrm{S}_{1}$ NHxInd (IRC point 1 toward isomer 1), gas phase

$\begin{array}{lrrr}\mathrm{C} & -2.02534653 & -2.34796043 & 0.22568407 \\ \mathrm{C} & -2.73184653 & -3.52888343 & 0.35473607 \\ \mathrm{C} & -4.06243153 & -3.54825643 & -0.05469893 \\ \mathrm{C} & -4.68468353 & -2.40200043 & -0.59019593 \\ \mathrm{C} & -3.99817653 & -1.21341443 & -0.72874293 \\ \mathrm{C} & -2.65406853 & -1.19075843 & -0.31559793 \\ \mathrm{H} & -2.25233253 & -4.40896243 & 0.76678707 \\ \mathrm{H} & -4.63829353 & -4.46171643 & 0.03875507 \\ \mathrm{H} & -5.72205953 & -2.46003043 & -0.89752993 \\ \mathrm{H} & -4.46538953 & -0.32692643 & -1.14029493 \\ \mathrm{C} & -0.63324653 & -1.99591543 & 0.55390007 \\ \mathrm{~N} & -1.78470753 & -0.16753443 & -0.34645993 \\ \mathrm{C} & -0.55270653 & -0.58958643 & 0.15882707 \\ \mathrm{C} & 0.41555347 & 0.41065657 & 0.13009507 \\ \mathrm{H} & -1.68201253 & 1.03464457 & -0.70942193 \\ \mathrm{O} & 0.23132447 & -2.73113043 & 1.03635207 \\ \mathrm{C} & 0.09839747 & 1.71900657 & -0.41020493 \\ \mathrm{~N} & 1.73215647 & 0.41664657 & 0.54094607 \\ \mathrm{C} & 2.28001847 & 1.65263557 & 0.25184407 \\ \mathrm{C} & 1.30196447 & 2.49205457 & -0.32032793 \\ \mathrm{O} & -1.04015953 & 2.06784857 & -0.86690393 \\ \mathrm{C} & 3.58025347 & 2.10806957 & 0.45165007 \\ \mathrm{C} & 1.60895547 & 3.80296357 & -0.6855593 \\ \mathrm{C} & 3.86725547 & 3.41439257 & 0.08075207 \\ \mathrm{C} & 2.89829547 & 4.25925657 & -0.47976893 \\ \mathrm{H} & 4.34738347 & 1.47158157 & 0.87479607 \\ \mathrm{H} & 4.87361947 & 3.79038457 & 0.22739607 \\ \mathrm{H} & 3.16742947 & 5.27180457 & -0.75454893 \\ \mathrm{H} & 0.84520947 & 4.43557457 & -1.12208593 \\ \mathrm{C} & 2.49327247 & -0.71820643 & 1.04190807 \\ \mathrm{C} & 3.14529447 & -1.51828143 & -0.07948693 \\ \mathrm{H} & 1.81227847 & -1.36384343 & 1.59538907 \\ \mathrm{H} & 3.24003347 & -0.32723943 & 1.73727307 \\ \mathrm{H} & 2.37722747 & -1.94695543 & -0.72591293 \\ \mathrm{H} & 3.81122447 & -0.89409343 & -0.68126693 \\ \mathrm{H} & 3.72389747 & -2.34290143 & 0.34266207\end{array}$


$\mathrm{S}_{1}$ NHxInd (IRC point 20 toward isomer 1), gas phase

$\begin{array}{lrrr}\mathrm{C} & -2.03087700 & -2.35231000 & 0.22573900 \\ \mathrm{C} & -2.73559300 & -3.53152600 & 0.35429800 \\ \mathrm{C} & -4.06808500 & -3.55070500 & -0.05577900 \\ \mathrm{C} & -4.68921800 & -2.41066900 & -0.58909700 \\ \mathrm{C} & -4.00031200 & -1.21794300 & -0.72819700 \\ \mathrm{C} & -2.66965300 & -1.21456000 & -0.31291300 \\ \mathrm{H} & -2.25620500 & -4.41145200 & 0.76656200 \\ \mathrm{H} & -4.64210900 & -4.46492600 & 0.03893100 \\ \mathrm{H} & -5.72641800 & -2.46735800 & -0.89682800 \\ \mathrm{H} & -4.46464700 & -0.33014700 & -1.14015400 \\ \mathrm{C} & -0.63509000 & -1.99706500 & 0.55343100 \\ \mathrm{~N} & -1.78051900 & -0.20121500 & -0.33268400 \\ \mathrm{C} & -0.54432100 & -0.58931500 & 0.16088700 \\ \mathrm{C} & 0.41390300 & 0.41320000 & 0.12802200 \\ \mathrm{H} & -1.87895000 & 0.77934700 & -0.67088100 \\ \mathrm{O} & 0.22899800 & -2.72924400 & 1.03473700 \\ \mathrm{C} & 0.08593300 & 1.73921900 & -0.42013500 \\ \mathrm{~N} & 1.72523100 & 0.41633600 & 0.53619600 \\ \mathrm{C} & 2.28523000 & 1.65978700 & 0.25101600 \\ \mathrm{C} & 1.31375700 & 2.49775900 & -0.31785800 \\ \mathrm{O} & -1.00854300 & 2.11738700 & -0.87412100 \\ \mathrm{C} & 3.58241000 & 2.10767400 & 0.45260100 \\ \mathrm{C} & 1.61160500 & 3.80080700 & -0.68424100 \\ \mathrm{C} & 3.87189100 & 3.41829000 & 0.08065600 \\ \mathrm{C} & 2.90532100 & 4.25957300 & -0.47802500 \\ \mathrm{H} & 4.34983400 & 1.47157700 & 0.87613600 \\ \mathrm{H} & 4.87828800 & 3.79299300 & 0.22812700 \\ \mathrm{H} & 3.17340500 & 5.27256700 & -0.75291800 \\ \mathrm{H} & 0.84320000 & 4.42777200 & -1.12083300 \\ \mathrm{C} & 2.48855800 & -0.71606800 & 1.04178500 \\ \mathrm{C} & 3.14330600 & -1.51699300 & -0.07984500 \\ \mathrm{H} & 1.80697500 & -1.36397600 & 1.59339600 \\ \mathrm{H} & 3.23504600 & -0.32043200 & 1.73443400 \\ \mathrm{H} & 2.37614800 & -1.94723400 & -0.72605300 \\ \mathrm{H} & 3.80985700 & -0.89280600 & -0.68046900 \\ \mathrm{H} & 3.72113500 & -2.33968900 & 0.34674200\end{array}$


$\mathrm{S}_{1}$ NHxInd (IRC point 40 toward isomer 1), gas phase

$\begin{array}{lrrr}\mathrm{C} & -2.03695500 & -2.37103600 & 0.23072400 \\ \mathrm{C} & -2.73968400 & -3.55630900 & 0.36021600 \\ \mathrm{C} & -4.07327300 & -3.58536900 & -0.04770600 \\ \mathrm{C} & -4.69997000 & -2.45014500 & -0.58067700 \\ \mathrm{C} & -4.01362400 & -1.25384400 & -0.71893800 \\ \mathrm{C} & -2.68418300 & -1.24045500 & -0.30553200 \\ \mathrm{H} & -2.25675900 & -4.43488400 & 0.77154200 \\ \mathrm{H} & -4.64070000 & -4.50356500 & 0.04863700 \\ \mathrm{H} & -5.73692500 & -2.50962400 & -0.88904000 \\ \mathrm{H} & -4.48289700 & -0.36863000 & -1.13120800 \\ \mathrm{C} & -0.64065000 & -2.00026800 & 0.55015200 \\ \mathrm{~N} & -1.79883000 & -0.21120500 & -0.33032900 \\ \mathrm{C} & -0.55708200 & -0.58073400 & 0.15196900 \\ \mathrm{C} & 0.39938900 & 0.43317600 & 0.11191100 \\ \mathrm{H} & -1.92584400 & 0.75734500 & -0.67064800 \\ \mathrm{O} & 0.23024300 & -2.72407500 & 1.02976100 \\ \mathrm{C} & 0.09127400 & 1.77310700 & -0.43051100 \\ \mathrm{~N} & 1.71295200 & 0.42691000 & 0.52105600 \\ \mathrm{C} & 2.29213500 & 1.66596800 & 0.24914500 \\ \mathrm{C} & 1.33240700 & 2.51582800 & -0.31779300 \\ \mathrm{O} & -0.98875200 & 2.17857900 & -0.88662400 \\ \mathrm{C} & 3.59418800 & 2.10105500 & 0.45810200 \\ \mathrm{C} & 1.64412900 & 3.81635200 & -0.67941600 \\ \mathrm{C} & 3.89860500 & 3.41065500 & 0.09163800 \\ \mathrm{C} & 2.94213400 & 4.26260300 & -0.46826400 \\ \mathrm{H} & 4.35465500 & 1.45669100 & 0.88183900 \\ \mathrm{H} & 4.90805200 & 3.77479900 & 0.24421400 \\ \mathrm{H} & 3.22045700 & 5.27380900 & -0.73976900 \\ \mathrm{H} & 0.88241900 & 4.45112600 & -1.11644700 \\ \mathrm{C} & 2.46373000 & -0.70784800 & 1.03690900 \\ \mathrm{C} & 3.12843000 & -1.51095000 & -0.07758700 \\ \mathrm{H} & 1.77395600 & -1.35393900 & 1.58038800 \\ \mathrm{H} & 3.20445100 & -0.31424500 & 1.73679600 \\ \mathrm{H} & 2.36756100 & -1.94189600 & -0.73059100 \\ \mathrm{H} & 3.80129100 & -0.88793600 & -0.67228000 \\ \mathrm{H} & 3.70136500 & -2.33287300 & 0.35681500\end{array}$


$\mathrm{S}_{1}$ NHxInd (IRC point 60 toward isomer 1), gas phase

$\begin{array}{lrrr}\mathrm{C} & -2.03559600 & -2.36820900 & 0.22944900 \\ \mathrm{C} & -2.71562700 & -3.56618100 & 0.36856700 \\ \mathrm{C} & -4.05033200 & -3.62122800 & -0.02977700 \\ \mathrm{C} & -4.69686200 & -2.49735200 & -0.56178000 \\ \mathrm{C} & -4.03148800 & -1.28863300 & -0.70867000 \\ \mathrm{C} & -2.69991000 & -1.24844400 & -0.30534800 \\ \mathrm{H} & -2.21202100 & -4.43314800 & 0.77978900 \\ \mathrm{H} & -4.60185900 & -4.54835200 & 0.07296500 \\ \mathrm{H} & -5.73465900 & -2.57359600 & -0.86383100 \\ \mathrm{H} & -4.52138600 & -0.41487700 & -1.12148100 \\ \mathrm{C} & -0.64453800 & -1.98167500 & 0.53787000 \\ \mathrm{~N} & -1.82382400 & -0.20481300 & -0.33852000 \\ \mathrm{C} & -0.57592300 & -0.55990500 & 0.13624600 \\ \mathrm{C} & 0.38287900 & 0.45327900 & 0.09650700 \\ \mathrm{H} & -1.96678800 & 0.75749300 & -0.67930200 \\ \mathrm{O} & 0.23481500 & -2.69815000 & 1.01310600 \\ \mathrm{C} & 0.08999200 & 1.79857800 & -0.44157900 \\ \mathrm{~N} & 1.69534300 & 0.43384100 & 0.51068200 \\ \mathrm{C} & 2.28910500 & 1.66617900 & 0.24654300 \\ \mathrm{C} & 1.34207400 & 2.53007800 & -0.32122300 \\ \mathrm{O} & -0.98627600 & 2.21077900 & -0.89688200 \\ \mathrm{C} & 3.59724600 & 2.07826400 & 0.46569900 \\ \mathrm{C} & 1.67951000 & 3.82760300 & -0.67260700 \\ \mathrm{C} & 3.92566800 & 3.38414400 & 0.10949900 \\ \mathrm{C} & 2.98407100 & 4.25225900 & -0.45059800 \\ \mathrm{H} & 4.34412300 & 1.41836600 & 0.88942800 \\ \mathrm{H} & 4.93961900 & 3.73191200 & 0.26978700 \\ \mathrm{H} & 3.27833800 & 5.26097100 & -0.71465100 \\ \mathrm{H} & 0.93280400 & 4.47941000 & -1.11049100 \\ \mathrm{C} & 2.43678600 & -0.70215900 & 1.03538900 \\ \mathrm{C} & 3.10403100 & -1.51049200 & -0.07374900 \\ \mathrm{H} & 1.74197200 & -1.34426000 & 1.57619000 \\ \mathrm{H} & 3.17537400 & -0.30898200 & 1.73755100 \\ \mathrm{H} & 2.34505300 & -1.93627500 & -0.73212700 \\ \mathrm{H} & 3.78615500 & -0.89263000 & -0.66325000 \\ \mathrm{H} & 3.66711700 & -2.33635500 & 0.36588300\end{array}$


$\mathrm{S}_{1}$ NHxInd (IRC point 80 toward isomer 1), gas phase

$\begin{array}{lrrr}\mathrm{C} & -2.03033500 & -2.36320100 & 0.22353700 \\ \mathrm{C} & -2.68703300 & -3.57288200 & 0.37282000 \\ \mathrm{C} & -4.02390500 & -3.65163000 & -0.01061100 \\ \mathrm{C} & -4.69138600 & -2.53824900 & -0.53763100 \\ \mathrm{C} & -4.04727800 & -1.31789200 & -0.69457200 \\ \mathrm{C} & -2.71277700 & -1.25289700 & -0.30582800 \\ \mathrm{H} & -2.16206800 & -4.42864300 & 0.78082700 \\ \mathrm{H} & -4.56006400 & -4.58683900 & 0.09968900 \\ \mathrm{H} & -5.73102200 & -2.63040300 & -0.82895600 \\ \mathrm{H} & -4.55832900 & -0.45495900 & -1.10456400 \\ \mathrm{C} & -0.64435800 & -1.96247900 & 0.51908300 \\ \mathrm{~N} & -1.84644000 & -0.19581000 & -0.34620700 \\ \mathrm{C} & -0.59263300 & -0.53902800 & 0.11922000 \\ \mathrm{C} & 0.36816100 & 0.47227000 & 0.08288300 \\ \mathrm{H} & -2.00267500 & 0.76176700 & -0.68693800 \\ \mathrm{O} & 0.24525900 & -2.67250400 & 0.98513800 \\ \mathrm{C} & 0.08941700 & 1.82058700 & -0.45262700 \\ \mathrm{~N} & 1.67842900 & 0.44039300 & 0.50515000 \\ \mathrm{C} & 2.28521200 & 1.66609700 & 0.24728900 \\ \mathrm{C} & 1.35025900 & 2.54178300 & -0.32344200 \\ \mathrm{O} & -0.98262400 & 2.23774800 & -0.91083700 \\ \mathrm{C} & 3.59811600 & 2.05795600 & 0.47532800 \\ \mathrm{C} & 1.70996200 & 3.83538000 & -0.66779000 \\ \mathrm{C} & 3.94746900 & 3.35954500 & 0.12620600 \\ \mathrm{C} & 3.01969400 & 4.24102900 & -0.43587500 \\ \mathrm{H} & 4.33297100 & 1.38538400 & 0.89977100 \\ \mathrm{H} & 4.96508800 & 3.69302800 & 0.29304400 \\ \mathrm{H} & 3.32839700 & 5.24681600 & -0.69466000 \\ \mathrm{H} & 0.97690300 & 4.50083600 & -1.10818500 \\ \mathrm{C} & 2.40975800 & -0.69654500 & 1.04067600 \\ \mathrm{C} & 3.06422800 & -1.52357700 & -0.06217400 \\ \mathrm{H} & 1.71173500 & -1.32650100 & 1.59065000 \\ \mathrm{H} & 3.15472700 & -0.30212500 & 1.73491400 \\ \mathrm{H} & 2.29841800 & -1.94766200 & -0.71330200 \\ \mathrm{H} & 3.75203700 & -0.91919000 & -0.65904600 \\ \mathrm{H} & 3.61841100 & -2.35146000 & 0.38481500 \\ & & & \end{array}$




$\begin{array}{lrrr}\mathrm{C} & -2.02965000 & -2.36281800 & 0.22242800 \\ \mathrm{C} & -2.68422700 & -3.57355200 & 0.37244400 \\ \mathrm{C} & -4.02150600 & -3.65414000 & -0.00885400 \\ \mathrm{C} & -4.69109600 & -2.54142000 & -0.53450100 \\ \mathrm{C} & -4.04890500 & -1.32003500 & -0.69223300 \\ \mathrm{C} & -2.71392200 & -1.25310400 & -0.30558900 \\ \mathrm{H} & -2.15718000 & -4.42850200 & 0.77947400 \\ \mathrm{H} & -4.55629500 & -4.59005900 & 0.10205400 \\ \mathrm{H} & -5.73108000 & -2.63478300 & -0.82421900 \\ \mathrm{H} & -4.56202300 & -0.45786300 & -1.10125300 \\ \mathrm{C} & -0.64400600 & -1.96101200 & 0.51640500 \\ \mathrm{~N} & -1.84846900 & -0.19483200 & -0.34653500 \\ \mathrm{C} & -0.59394800 & -0.53721400 & 0.11739100 \\ \mathrm{C} & 0.36696800 & 0.47396100 & 0.08144900 \\ \mathrm{H} & -2.00589700 & 0.76232400 & -0.68720500 \\ \mathrm{O} & 0.24667900 & -2.67070100 & 0.98094700 \\ \mathrm{C} & 0.08957000 & 1.82245300 & -0.45402800 \\ \mathrm{~N} & 1.67692000 & 0.44108600 & 0.50483800 \\ \mathrm{C} & 2.28488900 & 1.66619500 & 0.24756900 \\ \mathrm{C} & 1.35111400 & 2.54277400 & -0.32381800 \\ \mathrm{O} & -0.98194400 & 2.24003600 & -0.91285900 \\ \mathrm{C} & 3.59808900 & 2.05646300 & 0.47669400 \\ \mathrm{C} & 1.71273700 & 3.83594800 & -0.66776200 \\ \mathrm{C} & 3.94924800 & 3.35762200 & 0.12801000 \\ \mathrm{C} & 3.02280500 & 4.24008100 & -0.43472100 \\ \mathrm{H} & 4.33184700 & 1.38295800 & 0.90151900 \\ \mathrm{H} & 4.96710300 & 3.68996400 & 0.29564500 \\ \mathrm{H} & 3.33277900 & 5.24556200 & -0.69318300 \\ \mathrm{H} & 0.98093300 & 4.50240800 & -1.10871800 \\ \mathrm{C} & 2.40688700 & -0.69586900 & 1.04206600 \\ \mathrm{C} & 3.05964600 & -1.52577100 & -0.05961300 \\ \mathrm{H} & 1.70835900 & -1.32398600 & 1.59342600 \\ \mathrm{H} & 3.15270100 & -0.30119800 & 1.73520900 \\ \mathrm{H} & 2.29304600 & -1.94907400 & -0.71024400 \\ \mathrm{H} & 3.74891700 & -0.92361500 & -0.65705800 \\ \mathrm{H} & 3.61184800 & -2.35430200 & 0.38861200 \\ & & & \end{array}$

